\title{
HYDROLOGIC DATA OF THE NASHUA AND SOUHEGAN RIVER BASINS, MASSACHUSETTS
}

\author{
By
}

Bruce P. Hansen, R. A. Brackley, and Virginia de Lima

U.S. GEOLOGICAL SURVEY OPEN-FILE REPORT 87-221

Records of surface-water discharges, selected wells and borings, and chemical analyses of water in the Nashua and Souhegan River basins, Massachusetts

Prepared in cooperation with the

MASSACHUSETTS DEPARTMENT OF ENVIRONMENTAL MANAGEMENT

DIVISION OF WATER RESOURCES

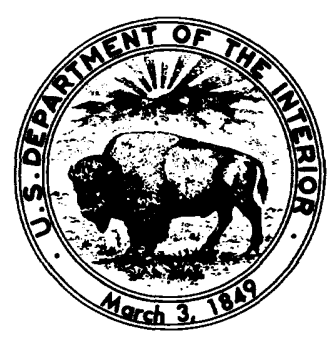

Boston, Massachusetts 1989 


\section{DEPARTMENT OF THE INTERIOR}

MANUEL LUJAN, JR., Secretary

\section{U.S. GEOLOGICAL SURVEY}

Dallas L. Peck, Director

District Chief

Water Resources Division

U.S. Geological Survey

10 Causeway Street, Suite 926

Boston, MA 02222-1040
U.S. Geological Survey

Books and Open-File Reports Section

Federal Center, Bldg. 41

Box 25425,

Denver, CO 80225 


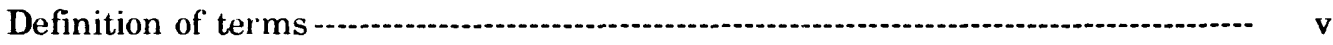

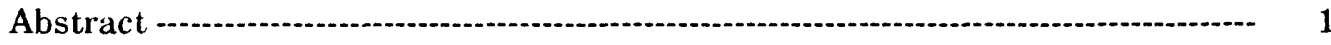

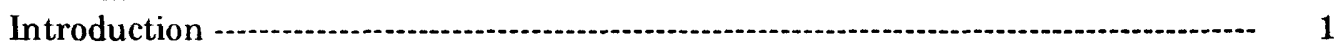

Numbering and location of hydrologic-data-collection sites -............................. 2

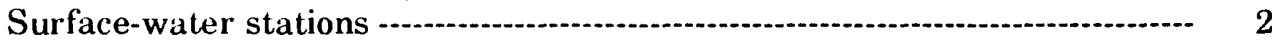

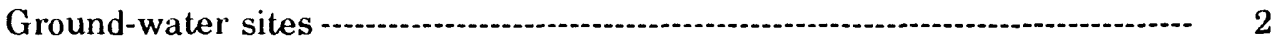

Collection and examination of hydrologic data -

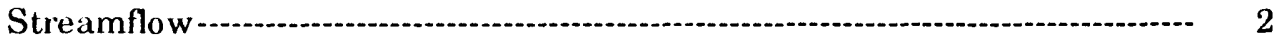

Solutes -

Temperature-1......-.

Selected references

\section{ILLUSTRATIONS}

Plate is in pocket

'late 1. Map of the Nashua and Souhegan River basins, Massachusetts, showing hydrologic-data-collection sites.

\section{TABLES}

1A. Description of selected wells and borings, data collected 1983-1986 -...................... 27

2. Logs of selected wells and borings, data collected 1971-1974

2A. Logs of selected wells and borings, data collected 1983-1986

3. Chemical analyses of ground water

4. Chemical analyses of precipitation, October 29, 1973

5. Water levels in observation wells

6. Stream sites and discharge measurements

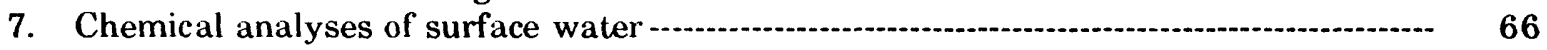

8. List of hydrologic-data reports for Massachusetts - 72 


\section{CONVERSION FACTORS}

The following factors may be used to convert inch-pound units published herein to metric (International System) units.

Multiply inch-pound units

By

To obtain metric units

\section{Length}

inch (in.)

$25.4 *$

millimeter $(\mathrm{mm})$

foot $(f t)$

0.30

meter $(\mathrm{m})$

mile (mi)

1.6

kilometer $(\mathrm{km})$

Area

square mile $\left(\mathrm{mi}^{2}\right)$

2.590

square kilometer $\left(\mathrm{km}^{2}\right)$

Flow

cubic foot per second $\left(\mathrm{ft}^{3} / \mathrm{s}\right)$

0.02832

cubic meter per second $\left(\mathrm{m}^{3} / \mathrm{s}\right)$

cubic foot per second per square

0.01093 $\operatorname{mile}\left[\left(\mathrm{ft}^{3} / \mathrm{s}\right) / \mathrm{mi}^{2}\right]$

gallon per minute (gal/min)

0.06308

cubic meter per second per square kilometer $\left[\left(\mathrm{m}^{3} / \mathrm{s}\right) / \mathrm{mi}^{2}\right]$

liter per second $(\mathrm{L} / \mathrm{s})$

Sea Level: In this report "sea level" refers to the National Geodetic Vertical Datum of 1929 (NGVD of 1929)-a geodetic datum derived from a general adjustment of the first-order level nets of both the United States and Canada, formerly called "Mean Sea Level of 1929".

* Exact. 


\section{DEFINITION OF TERMS}

Definition of terms related to streamflow, water quality, and other hydrologic data, as used in this report, are defined as follows:

Color is expressed in units of the platinum-cobalt scale proposed by Hazen (1892, p. 427-428). A unit of color is produced by 1 milligram per liter of platinum in the form of the chloroplatinate ion.

The extent to which water is colored by material in solution is reported as part of the water analysis because a significant color in water may indicate the presence of organic material that may have some bearing on the dissolved-solids content.

Cubic feet per second per square mile is the average number of cubic feet of water flowing per second from each square mile of area drained, assuming that the runoff is distributed uniformly in time and area.

Cubic foot per second $\left(\mathrm{ft}^{3} / \mathrm{s}\right)$ is the rate of discharge representing a volume of 1 cubic foot passing a given point during 1 second and is equivalent to 7.482 gallons per second, 448.8 gallons per minute, or 646,317 gallons per day.

Discharge is the volume of water (or more broadly, total fluids) that passes a given point within a given period of time.

Instantaneous discharge is the discharge at a particular instant of time and the column is labeled "Discharge $\left(\mathrm{ft}^{2} / \mathrm{s}\right)$ ".

Drainage area of a stream at a specified location is that area, measured in a horizontal plane enclosed by a topographic divide, from which direct surface runoff from precipitation normally drains by gravity into the stream above the specified point. Figures of drainage area given herein include all closed basins, or noncontributing areas, within the area unless otherwise noted.

Gage height is the water-surface elevation referred to some arbitrary gage datum.

Gaging station is a particular site on a stream where systematic observations of gage height or discharge are obtained. When used in connection with a discharge record, the term is applied only to those gaging stations where a continuous record of discharge is obtained.

Hardness of water is a physical-chemical characteristic attributable to the presence of alkaline earths (principally calcium and magnesium) and is expressed as equivalent calcium carbonate $\left(\mathrm{CaCO}^{3}\right)$. If the hardness exceeds the alkalinity (in milligrams per liter of $\mathrm{CaCO}_{3}$ or other equivalent units), the excess is termed "noncarbonate hardness".

Micrograms per liter $(\mu \mathrm{g} / \mathrm{L}, \mathrm{UG} / \mathrm{L})$ is a precise unit for expressing the concentration of chemical constituents in solution. One thousand micrograms per liter is equivalent to $1 \mathrm{milli}-$ gram per liter. See the following page. 
Milligrams per liter (mg/L, MG/L) is a unit for expressing the concentration of chemical constituents in solution. Milligrams per liter represents the weight of solute per unit volume of water. Milligrams or micrograms per liter may be converted to milliequivalents (one thousandth of a gram-equivalent weight of a constituent) per liter by multiplying by the factors in the table below. Concentration of suspended sediment expressed in milligrams per liter is based on the weight of sediment in a liter of water-sediment mixture.

\begin{tabular}{|c|c|c|c|}
\hline Ion & $\begin{array}{c}\text { Multiply } \\
\text { by }\end{array}$ & Ion & $\begin{array}{c}\text { Mul tiply } \\
\text { by }\end{array}$ \\
\hline $\begin{array}{l}\text { Ammonia as }\left(\mathrm{NH}_{4}{ }^{+1}\right) \\
\text { Bicarbonate }\left(\mathrm{HCO}_{3}{ }^{-1}\right) \\
\text { Calcium }\left(\mathrm{Ca}^{+2}\right) \\
\text { Carbonate }\left(\mathrm{CO}_{3}^{-2}\right) \\
\text { Chloride }\left(\mathrm{Cl}^{-1}\right)^{-} \\
\text {Copper }\left(\mathrm{Cu}^{+2}\right)^{*} \\
\text { Fluoride }\left(\mathrm{F}^{-1}\right) \\
\text { Hydrogen }\left(\mathrm{H}^{+1}\right)\end{array}$ & $\begin{array}{r}0.05544 \\
.01639 \\
.04990 \\
.03333 \\
.02821 \\
.03148 \\
.05264 \\
.99216\end{array}$ & $\begin{array}{l}\text { Iron }\left(\mathrm{Fe}^{+3}\right) * \\
\text { Magnesium }\left(\mathrm{Mg}^{+2}\right) \\
\text { Manganese }\left(\mathrm{Mn}^{+2}\right) \\
\text { Nitrate }\left(\mathrm{NO}_{3}^{-1}\right) \\
\text { Nitrite }\left(\mathrm{NO}_{2}^{-1}\right) \\
\text { Potassium }\left(\mathrm{K}^{+1}\right) \\
\text { Sodium }\left(\mathrm{Na}^{+1}\right) \\
\text { Sulfate }\left(\mathrm{SO}_{4}^{-2}\right)\end{array}$ & $\begin{array}{r}0.05372 \\
.08226 \\
.03640 \\
.01613 \\
.02174 \\
.02557 \\
.04350 \\
.02082\end{array}$ \\
\hline
\end{tabular}

*Constituent reported in micrograms per liter; multiply by factor and divide results by 1,000 .

$\mathrm{pH}$ is a symbol denoting the relative concentration of hydrogen ions in a solution; $\mathrm{pH}$ values range from 0 to 14 -the lower the value, the more acidic is the solution; that is, the more hydrogen ions it contains.

Refusal is a drilling term indicating the depth of a drill hole at which further penetration is impossible or impractical with the equipment being used.

Sediment is solid material that originates mostly from disintegrated rocks and is transported by, suspended in, or deposited from water; it includes chemical and biochemical precipitates and decomposed organic material such as humus. The quantity, characteristics, and cause of the occurrence of sediment in streams are influenced by environmental factors. Some major factors are degree of slope, length of slope, soil characteristics, land usage, and quantity and intensity of precipitation.

Solute is any substance derived from the atmosphere, vegetation, soil, or rocks that is dissolved in water.

Specific conductance is a measure of the ability of a water to conduct an electrical current and is expressed in micromsiemens per centimeter at 25 degrees Celsius. Because the specific conductance is related to the number and specific chemical types of ions in solution, it can be used for approximating the dissolved-solids content in the water. Commonly, the amount of dissolved solids (in milligrams per liter) is about 65 percent of the specific conductance (in micromhos). This relation is not constant from stream to stream or from well to well, and it may even vary in the same source with changes in the composition of the water. 


\title{
HYDROLOGIC DATA OF THE NASHUA AND SOUHEGAN RIVER BASINS, MASSACHUSETTS
}

\author{
By
}

Bruce P. Hansen, R. A. Brackley, and Virginia de Lima

\begin{abstract}
This report presents, in tabular form, hydrologic data collected during an investigation of water resources in the parts of the Nashua River basin and the Souhegan River basin which lie in Massachusetts.

Data presented in this report include selected information on wells and test borings; streamflow records; and chemical analyses of surface water, ground water, and rainfall. A map at a scale of 1:48,000 shows the location of all data sites.
\end{abstract}

\section{INTRODUCTION}

The Nashua and Souhegan Rivers are tributary to the Merrimack River and are located in north-central Massachusetts and south-central New Hampshire. The area covered by this report is comprised of the parts of the Nashua River basin $\left(445 \mathrm{mi}^{2}\right)$ and the Souhegan River basin $\left(9 \mathrm{mi}^{2}\right)$ which lie in Massachusetts. This area includes all or parts of the cities and towns of Ashburnham, Ashby, Ayer, Bolton, Boylston, Clinton, Dunstable, Fitchburg, Gardner, Groton, Harvard, Holden, Lancaster, Leominster, Lunenburg, Paxton, Pepperell, Princeton, Rutland, Shirley, Sterling, Townsend, West Boylston, and Westminster.

The hydrologic data presented in this report were collected in the mid 1970's during two investigations of the water resources of the Nashua and Souhegan River basins. These investigations were conducted by the U.S. Geological Survey in cooperation with the Massachusetts Department of Environmental Management, Division of Water Resources. The data complement interpretive reports (Brackley and Hansen, 1977 and Virginia de Lima, in preparation.)

Data include selected information on wells and test borings, streamflow records, and results of chemical analyses of surface water, ground water, and rainfall. (See pl. 1 for locations of all hydrologic-data-collection sites.)

The authors wish to acknowledge the public officials, consulting firms, industrial concerns, well drillers, and individual homeowners who have given their time and information to this study. 


\title{
NUMBERING AND LOCATION OF HYDROLOGIC-DATA COLLECTION SITES
}

\author{
Surface-Water Stations
}

Records are listed in downstream direction along the main stream, and stations on tributaries are listed between stations on the main stream in the order in which those tributaries enter the main stream. Stations on tributaries entering above all mainstream stations are listed before the first mainstream station. Stations on tributaries to tributaries are listed in a similar manner. All stations are numbered consecutively in downstream order in this report.

\section{Ground-Water Sites}

The well-numbering system of the U.S. Geological Survey is based on the grid system of latitude and longitude. The number consists of 14 digits and 1 letter. The first six digits denote the degrees, minutes, and seconds of latitude followed by a letter denoting north or south. Seven digits following the letter denote degrees, minutes, and seconds of longitude. The last digit is a sequential number for wells within a 1-second grid. The system provides the geographic location of the well and a unique number for each well.

A local numbering system for wells and borings also is used in this report. The first letter indicates whether the hole is a well $(W)$, auger boring $(A)$, bridge boring $(B)$, roadway boring (R), or miscellaneous boring $(\mathrm{X})$; and the number indicates the order in which the well or boring was inventoried within the town. A separate series of numbers beginning with "1" is used within each town. In tables and on the map (pl. 1), only the number is shown beside well locations, or the number plus " $A$ ", "B", or "R" for borings within the designating town boundaries.

\section{COLLECTION AND EXAMINATION OF HYDROLOGIC DATA Streamflow}

The data collected at continuous-record gaging stations consist of records of stage and measurements of discharge. Records of stage are obtained either from graphic water-stage recorders that provide a continuous record of the fluctuations or from digital recorders that punch tape at 15-, 30-, or 60-minute intervals. Measurements of discharge are made with a current meter, using the general methods adopted by the Geological Survey on the basis of experience in stream gaging since 1888. These methods are described in standard textbooks on the measurement of stream discharge (see also SELECTED REFERENCES).

More detailed information than that published for the gaging stations, such as discharge measurements, gage-height record, and rating tables, is on file in the district office. The long-term gaging-station records (through 1967) have been analyzed to give several statistical summaries including (1) the number of days in each year that the daily discharge was between selected limits (duration tables); (2) the lowest mean discharge for selected numbers of consecutive days in each year; and (3) the highest mean discharge for selected numbers of consecutive days in each year.

Measurements of streamflow made at low-flow discharge stations are made during periods when streamflow is primarily from ground-water storage. These measurements, when correlated with the simultaneous discharge of a nearby stream where continuous records are available, will give a picture of the potential low flow of the stream. 
The methods of collecting and analyzing the water samples for determining the kinds and concentrations of solutes are described by Skougstad and others (1979). One sample may define adequately the water quality in a stream at a given time if the mixture of solutes throughout the stream cross section is homogeneous. However, the concentration of solutes at different locations in the cross section may differ widely, depending on the rates of water discharge, the source of material and the turbulence and mixing of the stream. Some streams must be sampled at several locations across the channel to determine accurately the solute load. Temperal variations in solute load are only defined by periodic sampling over a period of time.

Ground-water quality does not change significantly during short periods of time; infrequent sampling and analysis of ground water adequately define ground-water quality at a given site.

Solids are dissolved from the atmosphere by precipitation. The amount and type of solids may be affected by the source of airborne particles, the wind direction and velocity, and the rainfall intensity and duration. Samples of rainfall were collected during selected periods.

\section{Temperature}

Water temperatures are measured at most water-quality stations. In addition, water temperatures are taken concurrently with most discharge measurements at surface-water stations. Large streams have a small daily temperature change while small, shallow streams may have a daily range of several degrees and may follow closely the changes in air temperature. To convert temperature data shown in degrees Celsius $\left({ }^{\circ} \mathrm{C}\right)$ to degrees Fahrenheit $\left({ }^{\circ} \mathrm{F}\right)$ or vice versa, use the following formulas.

$$
\begin{aligned}
& { }^{\circ} \mathrm{F}=9 / 5\left({ }^{\circ} \mathrm{C}\right)+32 \\
& { }^{\circ} \mathrm{C}=5 / 9\left({ }^{\circ} \mathrm{F}-32\right)
\end{aligned}
$$

\section{SELECTED REFERENCES}

Brackley, R. A., and Hansen, B. P., 1977, Water resources of the Nashua and Souhegan River basins, Massachusetts: U.S. Geological Survey Hydrologic Investigations Atlas 276.

Carter, R. W., and Davidian, Jacob, 1968, General procedure for gaging streams: U.S. Geological Survey Techniques of Water-Resources Investigations, book 3, chap. A6, 13 p.

Colby, B. R., 1963, Fluvial sediments-a summary of source, transportation, deposition, and measurement of sediment discharge: U.S. Geological Survey Bulletin 1181-A, 47 p.

Corbett, D. M., and others, 1943, reprinted 1957, Stream-gaging procedure, a manual describing methods and practices of the Geological Survey: U.S. Geological Survey Water-Supply Paper 888, 245 p.

Hazen, Allen, 1892, A new color standard for natural waters: American Chemical Journal, v. 12 , p. $427-428$.

Hem, J. D., 1970, Study and interpretation of the chemical characteristics of natural water, 2d ed.: U.S. Geological Survey Water-Supply Paper 1473, 363 p. 
Lane, E. W., and others, 1947, Report of the Subcommittee on Sediment Terminology: American Geophysical Union Transactions, v. 28, no. 6, p. 936-938.

Langbein, W. B., and Iseri, K. T., 1960, General introduction and hydrologic definitions: U.S. Geological Survey Water-Supply Paper 1541-A, 29 p.

Rainwater, F. H., and Thatcher, L. L., 1960, Methods for collection and analysis of water samples: U.S. Geological Survey Water-Supply Paper 1454, 301 p.

Skougstad, M. W., Fishman, M. J., Friedman, L. C., Erdmann, D. E., and Duncan, S. S., eds., 1979, Methods for determination of inorganic substances in water and fluvial sediments: U.S. Geological Survey Techniques of Water-Resources Investigations, book 5, chap. A1, 626 p.

U.S. Geological Survey, 1954, Compilation of records of surface waters of the United States through September 1950, pt. 1-A, North Atlantic slope basins, Maine to Connecticut: U.S. Geological Survey Water-Supply Paper 1301, 380 p.

1964, Compilation of records of surface waters of the United States, October 1950 to September 1960, pt. 1-A, North Atlantic slope basins, Maine to Connecticut: U.S. Geological Survey Water-Supply Paper 1721, 317 p.

1966-75, Water resources data for Massachusetts, New Hampshire, Rhode Island, Vermont, 1965-74: Boston, Mass., issued annually.

1969, Surface-water supply of the United States, 1961-65, pt. 1, North Atlantic slope basins, Maine to Connecticut: U.S. Geological Survey Water-Supply Paper 1901, 1027 p.

1975, Surface-water supply of the United States, 1966-70, part 1, North Atlantic slope basins, Maine to Connecticut: U.S. Geological Survey Water-Supply Paper 2101, 1, 123 p.

1976-86, Water resources data for Massachusetts and New Hampshire, 1975-84: Boston, Mass., issued annually.

U.S. Inter-Agency Committee on Water Resources, Subcommittee on Sedimentation, A study of methods used in measurement and analysis of sediment loads in streams: St. Anthony Falls Hydraulic Laboratory, Minneapolis, Minn., published in separate volumes as follows:

1963, A summary of the work of the Federal Inter-Agency Sedimentation Project: Report S.

1963, Determinations of fluvial sediment discharge: Report 14, $151 \mathrm{p}$.

1966, Instruments and reports for fluvial sediment investigations, Federal Inter-Agency Sedimentation Project (catalog of Inter-Agency Sedimentation Project), 67 p. 
LOCAL WELL NUMBER: LETTER PREFIX INDICATES--A, U.S. GEOLOGICAL SURVEY AUGER BORING; B, BRIDGE BORING; R, ROADWAY BORING; $W$, WELL OR TEST WELL (THE "W" IS OMITTED FROM PLATE 1 TO CONSERVE SPACE); $X$, MISCELLANEOUS TEST BORING.

LATITUDE-LONGITUDE: NUMBER FOLLOWING DECIMAL POINT IS A SEQUENTIAL NUMBER FOR WELLS OR BORINGS IN A 1-SECOND GRID.

ALTITUDE OF LAND-SURFACE DATUM: ALTITUDES ARE EXPRESSED IN FEET ABOVE SEA LEVEL; THOSE PRECEOED BY A MINUS SIGN ARE BELOW SEA LEVEL.

METHOD DRILLED: A, AIR-ROTARY; B, BORED OR AUGERED; C, CABLE TOOL; D, DUG; H, HYDRAULIC-ROTARY; J, JETTED; P, AIRPERCUSSION; R, REVERSE-ROTARY; T, TRENCHED; V, DRIVEN; W, DRIVE-WASH.

WELL FINISH: C, POROUS CONCRETE; F, GRAVEL WALL WITH PERFORATED OR SLOTTED CASING; G, GRAVEL WALL WITH COMMERCIAL SCREEN; H, HORI ZONTAL GALLERY OR COLLECTOR; 0 , OPEN END; P, PERFORATED OR SLOTTED CASING; S, SCREEN; T, SAND POINT; W, WALLED OR SHORED; $X$, OPEN HOLE IN AQUIFER (GENERALLY CASED TO AQUIFER).

WELL DEPTH: DEPTH OF FINISHED WELL, IN FEET BELOW LAND SURFACE.

WELL USE: A, ANODE; D, DRAINAGE; G, SEISMIC HOLE; H, HEAT RESERVOIR; 0, OBSERVATION; P, OIL OR GAS; R, RECHARGE; T, TEST; $U$, UNUSED; $W$, WATER WITHDRAWAL; $X$, WASTE DISPOSAL; $Z$, DESTROYED.

WATER-BEARING MATERIAL: PRINCIPAL WATER-BEARING ZONE.
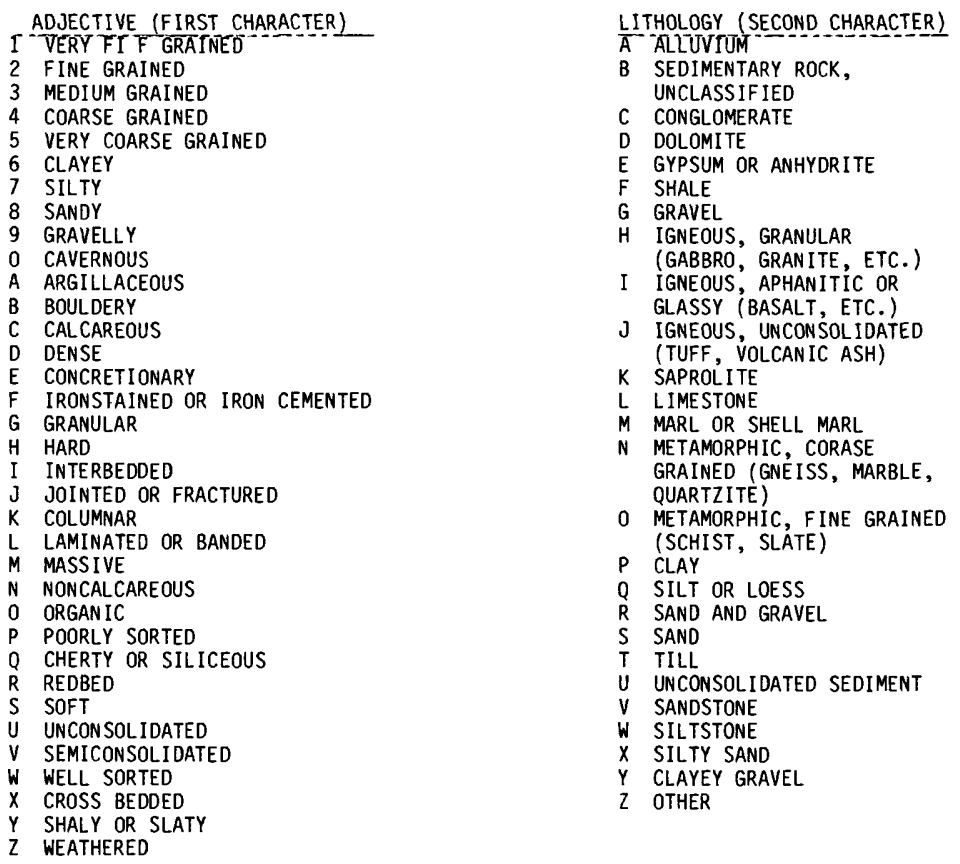

WATER LEVEL: LEVELS ARE GIVEN IN FEET BELOW LAND SURFACE; "+" INDICATES WATER LEVEL ABOVE LAND SURFACE; "F" INDICATES FLOWING WELL.

WATER USE: A, AIR CONDITIONING; B, BOTTLING; C, COMMERICAL; D, DEWATERING; E, POWER GENERATION; F, FIRE PROTECTION; H, DOMESTIC; I, IRR IGATION; M, MEDICINAL; $N$, INDUSTR IAL (INCLUDES MINING); $P$, PUBLIC SUPPLY; R, RECREATION; S, STOCK; T, INSTI TUTIONAL; U, UNUSED; $V$, REPRESSURIZÁTION; $W$, RECHARGE; $X$, DESALINATION--PUBLIC SUPPLIES; $Y$, DESALINATION --OTHER SUPPLIES.

PUMPAGE, YIELD: IN GALLONS PER MINUTE (GAL/MIN).

PUMPAGE, DRAWDOWN: THE DIFFERENCE BETWEEN STATIC WATER LEVEL AND PUMPING LEVEL.

PUMPAGE, TIME: THE FOLLOWING CODES ARE USED FOR PUMPING PERIODS OF LESS THAN 1 HOUR: A, THROUGH 15 MINUTES; B, 15 TO 30 MINUTES; $C, 30$ TO 45 MINUTES; D, 45 TO 59 MINUTES.

LOG: D, DRILLER'S LOG; E, ELECTRIC LOG; G, GEOLOGIST'S LOG AVAILABLE IN TABLE 2.

QW: TYPE OF CHEMICAL ANALYSIS AVAILABLE IN TABLE 3. C, COMPLETE; J, CONDUCTANCE AND CHLORIDE K, CONDUCTANCE;

L, CHLORIDE; M, MULTIPLE (INCLUDES ONE COMPLETE AND ONE OR MORE PARTIAL); P, PARTIAL. 
TABLE I.--DESCRIPTION OF SELECTED WELLS, TEST WELLS, AND BORINGS

[A dash indicates no data are available.]

LOCAL

WELL

LAT ITUDE-

ALTILONGITUDE OF LSD
OWNER OR USER

YEAR/ METHOD DIAM-TFIN-IDEPTHIUSE FEET WATERETER IISH :
(IN) I I I I YERTLE TTER IISH I BEARING

LEVEL IDATE
IMEAS- $-\overline{Y U S E}$
YIELDI DD ITIME LOG OW

ASHBURNHAM

423853N07I5737.I 1058 $\begin{array}{llr}1 & 423904 N 0715821 . I & 1063 \\ 2 & 423632 N 0715534.1 & 849\end{array}$ $\begin{array}{rrr}2 & 423632 N 0715534.1 & 849 \\ 2 & 424021 N 0715200.1 & 1285\end{array}$ $3424117 N 0715242.11130$

$4 \quad 424209 N 0715446.1 \quad 1175$ $\begin{array}{lll}5 & 424204 N 0715450.1 & 1185 \\ 6 & 424027 N 0715205.1 & 1305\end{array}$ $\begin{array}{ll}423959 N 0715342.1 & 1140 \\ 423927 N 0715444 . I & 1209\end{array}$

$9424001 N 0715342.1 \quad 1135$ $10423822 N 0715336.11150$ 11 $423827 \mathrm{NO} 715328.1$ I 155 13 423859N07I5311.1 1257

14 423841NO715150.1 I 255 I5 423840N0715501.1 I3I5 I6 423725N0715335.I I135 $\begin{array}{lll}17 & 423659 N 0715317.1 & 1145 \\ 18 & 423937 N 0715138.1 & 1200\end{array}$

$19442326 N 0715510.1848$ $\begin{array}{lll}20 & 423921 N 0715331.1 & 1149 \\ 21 & 423916 N 0715318.1 & 1165\end{array}$ 22 4238IIN0715534.1 II95 423933N0715900.1 1038

2423934 N07I5857.1 1040 3 423935N0715854.I I 030 $4 \quad 423936 N 0715852.11048$ 6 423622N0715538.1 870

$7423628 N 0715536.1860$ 423630 N0715611.1 965 10 4237ION0715559.1 1015 11 423720N0715541.1 1012

$12423743 \mathrm{~N} 0715438.1 \quad 1030$ $\begin{array}{lll}13 & 423759 N 0715438.1 & 985 \\ 14 & 423754 N 0715418.1 & 949\end{array}$ $\begin{array}{llr}14 & 423754 N 0715418.1 & 949 \\ 15 & 423811 N 0715457.1 & 1100\end{array}$ I6 423810NO715449.1 I055

$\times \quad 1742382$ IN0715432.I 1035

I 424023N0714749.I 730 $2424045 N 0714745.1 \quad 715$ 4 42402INO714747.1 712 5424220 NO 725322.1 I 1220

6 424I46N0115053.I IISS 7 424125N0715046.I 1064 424I23NOII5052.1 1055 $\begin{array}{rrr}9 & 424059 N 0715013.1 & 980 \\ 10 & 424037 N 0715034.1 & 1040\end{array}$

II $424009 N 0714928$. I 885 $\begin{array}{lll}12 & 424019 N 0714917.1 & 930 \\ 13 & 424112 N 0714906.1 & 800\end{array}$ $\begin{array}{rrr}14 & 423956 N 0715056.1 & 1210 \\ 15 & 424004 N 0714935.1 & 875\end{array}$

I6 424002N07I4930.1 900 $17424000 N 0714929.1 \quad 890$ $19 \quad 423919 N 0714741.1 \quad 670$

2I $423929 \mathrm{~N} 0714747.1 \quad 685$ 22 423932NO $114749.1 \quad 670$ $23424004 \mathrm{NO} 71490 \mathrm{I} . \mathrm{I} \quad 800$ 25 424150NO714715.I 655

26 424I01N0714756.I 755 $\begin{array}{lll}27 & 424034 N 0714648.1 & 565 \\ 28 & 424031 N 0714653.1 & 565\end{array}$ $\begin{array}{lll}28 & 424031 N 0714653.1 & 565 \\ 29 & 424055 N 0714754.1 & 725\end{array}$ 30 423946N0714740.I 645

$31423820 \mathrm{~N} 0714739.1 \quad 700$ $\begin{array}{lll}32 & 423824 N 07 I 4738 . I & 690 \\ 33 & 42383 I N 07 I 4738 . I & 695\end{array}$ $\begin{array}{lll}33 & 42383 \text { IN07I } 4738.1 & 695 \\ 34 & 424009 N 0714827.1 & 840\end{array}$ $35424020 N 0714843.1 \quad 860$

US GEOL SURVEY

MDP

WAAG OLAVI

JOHNSON EINO J

1966

SPORTSMAN CLUB

SPORTSMAN CLUB

AM TEL AND TEL

FRANCAVILLE S

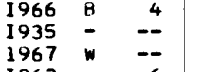

1963

CAMP WINNEKENG

\begin{tabular}{ccc|}
-- & $D$ & -- \\
$\overline{1967}$ & $D$ & - \\
-- & -
\end{tabular}

1967

$-\quad-\quad-$

SALONEN TOREKO

KALL INEN EDI TH

KIMBALL BRUC

-- $\quad-$

RICE JAMES $P$

1965
1967

THOMA MART IN

CLAUMS LEANDER

KENYON RESTHOME

OBRIEN FRANCIS

1968 A

MACMULLEN EDSDN I966 A

BURRAGEVILL DAM I 1969 V

BURRAGEVILL DAM 1969

BURRAGEVILL OAM I969 V

BURRAGEVILL DAM I 969

ASHBURNHAM TOWN $1963 \mathrm{~V}$

ASHBURNHAM TOWN I963

ASHBURNHAM TOWN I963V

ASHBURNHAM TOWN $1963 \mathrm{~V}$

ASHBURNHAM TOWN 1963

ASHBURNHAM TOWN $1963 \mathrm{~V}$

ASHBURNHAM TOWN 1963

ASHBURNHAM TOWN 1963

ASHBURNHAY TOWN 1963

ASHBURNHAM TOWN 1963

ASHBURNHAM TOWN 1963

ASHBURNHAM TOWN I963

ASHBURNHAM TOWN $1963 \mathrm{~V}$

WAYRANEN RAY

WAYRANEN HENRY

HARTU OTTO

WATATIG SKI

JOKI UNTO

LEMIEUR JOHN

OLKKLA OIVA

INGERSON HENRY

CUDMORE A OOUG

KULJU ANDREW

OODRUFF $W$

MYERS ARTHUR E

KAIVOLA

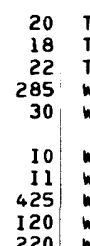

$\begin{array}{lr}-- & T \\ -- & -- \\ -- & 3 R \\ -- & U\end{array}$

12
--6
35

$\begin{array}{rr}1 I-66 & U \\ -- & U \\ 12-67 & U \\ 7-63 & S\end{array}$

(GPM) I (FT) । (HR)

MASON LEON

WALSH EDWARD

\begin{tabular}{lllr|ll} 
MICRO TOOL CO & 1967 & $R$ & 8 & $x$ & 110 \\
MISSLIN ALFRED & 1946 & - & -- & - & $I 08$
\end{tabular}

RAJALA JOHN

LANGILLE EOMUND

MORRILL $P$

VANVOOR8I5 B

MILLER

LAHTI HAROLD

$6 \quad 5$

35

$\begin{array}{ll}-- & -- \\ \text { II } & 0 \\ -- & --\end{array}$

$\begin{array}{ccc}=- & -- & H \\ -- & -- & H \\ 22 & 12-67 & C \\ =- & -- & H \\ -- & - & H\end{array}$

$\overline{--}$

$3 \overrightarrow{30}$

180

160

$\overline{--}$

--
$12-65$
$4-67$

$--$

145
205

\begin{tabular}{l|l} 
I & \\
I & \\
I & \\
2 & 0 \\
2 &
\end{tabular}

QUADRELLE $P$

STE INUS HUGO

COMEAU HOMEK N 1971

JOYCE FRANCIS

KOSKI WALTER

BEAL RAYMOND

CURRAN JAMES
LOMEGHO PHILIP
I 971
ASHBY

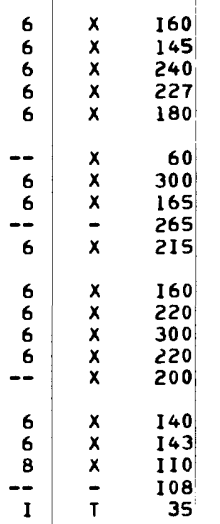

28

36

105

$51 W$ I5 -

200

156
200

200
20

$283 W \quad 20 \quad--\quad \overline{1}$

I0

101
$250 w$

345
200
100

$325 w$

$186 w$

$110 w$

$\begin{array}{rr}76 & -- \\ 8 & -- \\ 9 & --\end{array}$

$38 \quad T \quad-2 \quad 7 R$

\begin{tabular}{r|rll}
38 & $T$ & -- & $7 R$ \\
10 & $T$ & -- & $7 S$ \\
6 & $T$ & -- & -- \\
10 & $T$ & -- & --
\end{tabular}

10 T $=-$

$\begin{array}{llll}10 & T & -- & -- \\ 10 & T & -- & -\end{array}$

$\begin{array}{rlll}10 & 1 & = & =\end{array}$

$\begin{array}{llll}10 & T & -- & --\end{array}$

\begin{tabular}{r|r|}
10 & $T$ \\
10 & $T$ \\
10 & $T$ \\
8 & $T$ \\
8 &
\end{tabular}

$--$

$\begin{array}{ccccccc}-- & H & 3 & -- & 4 & - & P \\ 5-62 & H & 3 & -- & - & - & - \\ -- & H & 7 & -- & 4 & - & - \\ -- & H & -- & -- & -- & - & - \\ I-71 & H & -5 & -- & -- & - & - \\ -- & T & -- & -- & -- & - & P \\ 8-68 & H & 20 & -- & -- & - & - \\ 6-46 & H & 6 & -- & -- & - & - \\ 9-66 & H & 25 & -- & -- & - & - \\ 7-69 & U & -- & -- & -- & D & - \\ 8-69 & U & -- & -- & -- & 0 \\ 8-69 & U & -- & -- & -- & 0 \\ -- & U & -- & -- & -- & 0 & - \\ 5-63 & U & -- & -- & -- & D & - \\ 5-63 & U & -- & -- & -- & D & -\end{array}$

$5-63 u$

$\overline{--}=$

3-63 U

$\begin{array}{ll}3-63 & 0 \\ 5-63 & u\end{array}$

$\begin{array}{ll}5-63 & u \\ 5-63 & u\end{array}$

5-63 u

$\begin{array}{cc}3-63 & \\ -- & v\end{array}$

$--$

$8-64$ $2-64$
--
$0-63$

$10-63$

9-65 H

--

$8-65$

$7-66$

9-64

9-64 H

--
$7-72$
-46

$-46$

$-58$

--
$10-65$

$--$

$-65$

$=$

$\begin{array}{ll}-- & H \\ =- & H \\ -52 & H\end{array}$

$\overline{--}$ 


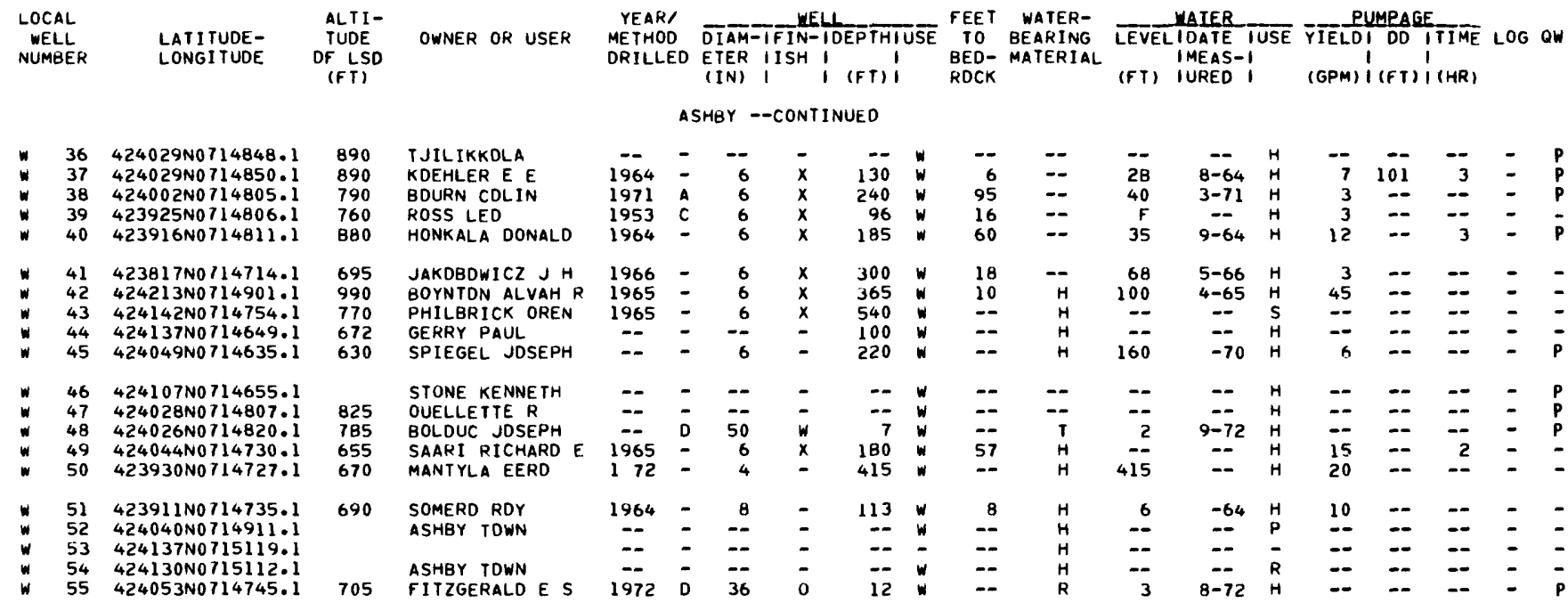

$1423331 \mathrm{N0} 713508.1$

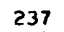

MOPW

2423331 NOT13508.2 241

3 42332BN0713603.1 226

$\begin{array}{lll}4 & 42330 B N 0713428.1 & 213 \\ 1 & 423333 N 0713336.1 & 230\end{array}$

$2423304 N 0713439.1230$

$3423309 N_{0} 713711.1$

4 423328N0713521.1

8 423305NO713453.1

$423305 N 0713453.2$

423448N0713632.2

$13423442 N 0713605.1$

$523310 N 0713701.1$
$7423412 N 0713549.1$

$18423448 N 0713601.1$

$423338 \times 0713516.1$

$423347 N 0713525.1$

423311 NOT13311.1

26 423337N0713309.1

$27423307 N 0713421.1$

28 423315N0113506.1

$39 \quad 423324 \mathrm{NO} 713519.1$

$40 \quad 423321 N 0713524.1$

41 423315N0713516.1

$423315 N 0713516.1$
$423327 N 0713525.1$
$423327 N 0713641.1$

230
208

225
220

230

230
220

220

227

220

235

220

231

225

220
230

218

218
224
212

422722NO713844.1 230

$18422723 N 0713639.1 \quad 530$

$19422729 N 0713638.1 \quad 485$

$\begin{array}{lll}43 & 422736 N 0713823.1 & 235\end{array}$

$44 \quad 422711 N 0713801.1 \quad 380$

$60422547 N 0713927.1310$

62 422659NO713759.1 385

63 422731NO713833.1

515

64422631 No713728.1

65 422715N0713837.1

$69422705 \mathrm{~N}_{0} 713709.1$

2 422759N0713757.1

$79422803 \mathrm{NO} / 13750.1$

80 422804N0713754.1

81 422800N0713750.1

$85422746 N 0713736.1$

97422639 NOIl $3724.1 \quad 450$

106422712 N0713706.1

109 422701N0713704.1

$11422703 \mathrm{NO} 713851$.1

$115422704 N 0713851.1$
$116 \quad 422707 N 0713849.1$

MDPW

MDPW

AYER TDWN

$\because \quad 0$

AYER TDWN

AYER ICE CO

HARTNETT TANNER

FDRT DEVENS

AYER TOWN

AYER TOWN

AYER TDWN

TDMS FDDD WDRLD

AYER GAME FARM

PRIEST H B

CDLD STORAGE CO

KLEENIT INC

AYER TOWN

AYER TOWN

$\begin{array}{ll} & 1952 \\ \text { AYER TOWN } & 1942\end{array}$

HARTNETT TANNER 1956

HARTNETT TANNER 1955

$\begin{array}{ll}\text { HARTNETT TANNER } & 1955 \\ \text { FORT DEVENS } & 1966\end{array}$

$\begin{array}{lll}1931 & W & -- \\ 1931 & - & -- \\ 1931 & - & -- \\ 1941 & - & - \\ 1942 & v & 2 \\ 1942 & - & -- \\ -- & v & 2 \\ -- & - & -- \\ -- & v & 2 \\ 1952 & C & 12\end{array}$

$\begin{array}{rrrr}19 & T & -- & -- \\ 42 & T & -- & -- \\ 36 & T & -- & -- \\ 48 & T & - & - \\ 41 & T & 41 & R \\ 62 & T & -- & R \\ 29 & W & 29 & R \\ 60 & W & -- & R \\ 75 & W & -- & R \\ 60 & T & -- & G \\ 60 & T & -- & 3 R \\ 24 & T & -- & 8 R \\ 36 & T & -- & -- \\ 29 & T & -- & R \\ 392 & W & 29 & --\end{array}$

$\begin{array}{llllllll}-- & - & U & -- & -- & -- & 0 & - \\ 13 & -31 & U & -- & -- & -- & 0 & - \\ -- & -- & U & - & -- & -- & 0 & - \\ -- & -- & U & -- & -- & -- & 0 & -\end{array}$

$290 \$ 15$

$\begin{array}{rrrr}152 & W & 0 & -- \\ 25 & T & 25 & S\end{array}$

$\begin{array}{llll}16 & T & 16 & 25 \\ 58 & T & -- & 86\end{array}$

$\begin{array}{ll}65 & 1 \\ 55 & 1\end{array}$

55
27
44

$\begin{array}{lr}-- & S \\ -- & R \\ 27 & R \\ -- & --\end{array}$

$\begin{array}{ccc}4 & 8-42 & \\ 10 & 10-58 & N \\ -- & -- & C \\ -- & -- & P\end{array}$

$\begin{array}{llll}00 & 3 & 32 & 0\end{array}$

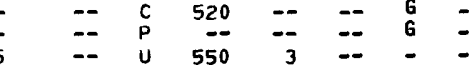

$\begin{array}{cccccccc}6 & 6-52 & U & 750 & -- & -- & 0 & - \\ -- & -- & U & -- & = & -- & D & = \\ - & -- & U & -- & -- & -- & 0 & - \\ 4 & 12-67 & U & -- & -- & -- & 0 & - \\ 4 & 3-58 & C & 5 & -- & -- & - & -\end{array}$

$\begin{array}{llllllll}8 & - & H & 10 & -- & -- & - & -\end{array}$

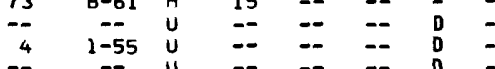

$\begin{array}{llllllll}-- & - & U & -- & -- & - & 0 & -\end{array}$

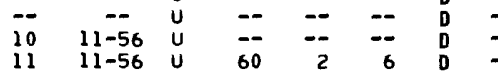

$\begin{array}{llll}58 & T & - & R\end{array}$

\section{BOLTON}

MOPW

TURNER PAUL

CRISPIN

BOLTDN TDWN

$\begin{array}{llr}1958 & \bar{C} & -\overline{6} \\ 1954 & C & 6 \\ 1954 & C & 6 \\ 1968 & W & 2\end{array}$

NASHOBA SCHDOL

PRESCOTT LEE R

RUNAWAY 8RK CC

DEERF OOT FARMS

$1960 \mathrm{~W}$

$1968 \mathrm{~A}$

$1956-12$

BOLTDN FRUIT CO 1959

BOLTDN FRUIT CO $1959=$

BOLTDN FRUIT CO

BARTLETT R A MD

CDMERFORD JDHN

CROSSMAN A J

DAY GEORGE

DURIVAGE $F$

CRAIN F $B$
HINES RICHARD

1953
1966
1961

PLATTER

RHODES SUSAN G 1969 -

TOBIN JOHN J 1966

LEOMINSTER CITY 1961

LEOMINSTER CITY 1961

$x$
6
6
6
6
6
6
6
6
6
6
$x$
$x$
$x$

$\begin{array}{rrrrr}70 & T & -- & 5 & \\ 90 & W & 33 & -- & 38 \\ 116 & W & 36 & -- & 15 \\ 264 & U & 41 & -- & 50 \\ 90 & T & -- & 9 x & \end{array}$

$\begin{array}{rrrr}68 & T & 70 & 6 S \\ 188 & W & 25 & -- \\ 128 & W & 128 & R \\ 300 & W & 39 & --\end{array}$

$128 \mathrm{~W}$

$\begin{array}{rrrr}195 & w & 53 & -- \\ 25 & w & -- & 5 \\ 600 & w & 6 & -- \\ 70 & w & -- & U\end{array}$

287 114

75
46
70
253
31

125

185
70

93

\begin{tabular}{|c|c|c|c|c|c|}
\hline $\begin{array}{r}-- \\
-58 \\
-54 \\
-54 \\
11-68\end{array}$ & $\begin{array}{l}U \\
H \\
H \\
H \\
U\end{array}$ & $\begin{array}{r}-- \\
5 \\
2 \\
1 \\
9\end{array}$ & $\begin{array}{l}=- \\
=- \\
=-\end{array}$ & $\begin{array}{l}=- \\
=- \\
=-\end{array}$ & $=$ \\
\hline $\begin{array}{r}3-60 \\
10-68 \\
1-56 \\
4-60 \\
11-49\end{array}$ & $\begin{array}{l}\text { U } \\
H \\
I \\
T \\
-\end{array}$ & $\begin{array}{r}0 \\
1230 \\
80 \\
--\end{array}$ & $\begin{array}{l}=- \\
=- \\
=-\end{array}$ & $\begin{array}{l}-- \\
-- \\
28 \\
24 \\
--\end{array}$ & 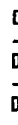 \\
\hline $\begin{array}{c}1-59 \\
8-53 \\
-- \\
-61 \\
9-51\end{array}$ & $\begin{array}{l}- \\
\bar{H} \\
H \\
H\end{array}$ & $\begin{array}{r}15 \\
20 \\
.5 \\
7 \\
30\end{array}$ & $\begin{array}{l}= \\
= \\
=\end{array}$ & $\begin{array}{r}-- \\
-- \\
--\end{array}$ & 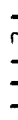 \\
\hline $\begin{array}{l}8-48 \\
1-44 \\
1-63 \\
8-54 \\
2-53\end{array}$ & $\begin{array}{l}H \\
H \\
H \\
H \\
H\end{array}$ & $\begin{array}{r}25 \\
25 \\
40 \\
9 \\
20\end{array}$ & $\begin{array}{l}= \\
=- \\
=\end{array}$ & $\begin{array}{l}-- \\
=- \\
--\end{array}$ & - \\
\hline $\begin{array}{c}4-69 \\
-- \\
4-60 \\
2-61 \\
2-61\end{array}$ & $\begin{array}{l}H \\
H \\
H \\
U \\
U\end{array}$ & $\begin{array}{r}25 \\
3 \\
15 \\
65 \\
65\end{array}$ & $\begin{array}{l}= \\
= \\
= \\
-\end{array}$ & $\begin{array}{l}=- \\
=- \\
--\end{array}$ & 0 \\
\hline
\end{tabular}


TABLE 1.--DESCRIPTION OF SELECTED WELLS, TEST WELLS, AND BORINGS -- CONTINUED

LDCAL

WELL LONGITUDE OF LSD
ALTI-
LATITUDE-
LONGITUDE
OWNER OR USER

\section{BOLTON --CONTINUED}

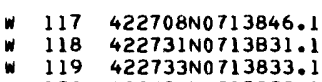

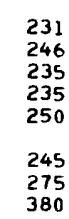
$119 \quad 422733 N 0713833.1$
$120 \quad 422734 N 0713828.1$ $120422734 N 0713828.1$
$121 \quad 422728 N 0713834.1$

w 122 422748N0713804.1 123
$W \quad 22753 N 0713750.1$
$W \quad 422750 N 0713732.1$

231
246
235
235
250
245
275
380

615

585
540

540
530

500

532

555

630

550
440

460

565

525
500
450

520

570

560

629
560

415

\section{LEOMINSTER CITY 1961 \\ LEOMINSTER CITY 1961 - \\ LEOMINSTER CITY $1961-$
LEOMINSTER CITY $1961-$ \\ SCHNEIDER HARRY $1968 \mathrm{C}$ \\ $\begin{array}{ll}\text { KUNST JOHN } & 1966 \\ \text { LEMAY RICHARD A } & --\end{array}$}

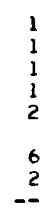
\begin{tabular}{r|rrr}
102 & $T$ & 101 & $8 R$ \\
127 & $T$ & 127 & $R$ \\
$B 7$ & $T$ & -7 & $8 P$ \\
125 & $T$ & 125 & $9 S$ \\
103 & $T$ & 103 & $R$
\end{tabular}

\begin{tabular}{r|rrr}
125 & $W$ & 75 & -- \\
60 & $W$ & -- & $R$
\end{tabular}

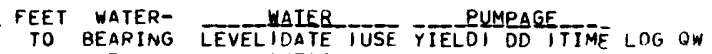
ROCK (FT) IURED I

(GPM) I (FT) । (HR)

ROSE JAMES $J$

PARKER ALBERT C $1963=$ SULLIVAN R JR 1955 C RE DAVIO $\begin{array}{ll}1951 & C \\ 1966-\end{array}$

POPE ALFRED $F$

ZEPP WARREN $B$

TAYLOR GEORGE

MOORE JOHN W

$1968 P$

1952 C

1967

OOD NELSON P 1957

GARF IELD H S

WILLIAMS F R 1950 C

MOORE JOHN W 1960 -

BRIGHAM HERBERT 1957 P

KASSAY ATTILA $1960 P$

CUTLER HOWARD B 1967 P

CHIARELLJ LUIGI 1943 C

$\begin{array}{lll}\text { WEAUER ROBERT R } & 1969 & \text { A } \\ \text { SPENCER KNOLLIN } 1964 & -\end{array}$

BOYLSTON W D

BOYLSTON

\begin{tabular}{l|}
-- \\
\hline 6 \\
6 \\
6 \\
6 \\
\hline 6 \\
6 \\
6 \\
6 \\
2 \\
\hline 6 \\
6 \\
6 \\
6 \\
6 \\
\hline 6 \\
6
\end{tabular}

$\begin{array}{rrrr}100 & w & -- & -- \\ 240 & w & 9 & = \\ 130 & w & 4 & -- \\ 102 & w & 8 & -- \\ 90 & w & 52 & --\end{array}$

$\begin{array}{rrrrrrrr}+1 & 2-61 & U & 60 & -- & -- & 0 & - \\ 21 & 3-61 & U & -- & -- & -- & 0 & - \\ -- & -- & U & -- & -- & -- & 0 & - \\ 6 & 2-61 & U & 60 & -- & -- & 0 & - \\ 22 & 3-61 & U & 45 & -- & -- & 0 & P \\ 25 & -68 & H & 20 & -- & -- & - & - \\ 21 & 6-61 & H & -- & -- & -- & - & - \\ -- & -- & H & -- & -- & -- & - & p\end{array}$

300

77
200
130

130
22

$13=-$

14

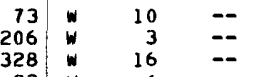

$93 W \quad 20 \quad-$

88 w

180

71
90

$102 W$

$\begin{array}{rr}4 & -- \\ 30 & -- \\ -- & - \\ 130 & H \\ 13 & H\end{array}$

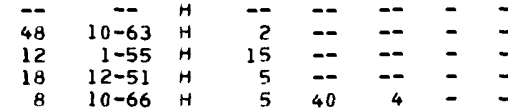

$78 w$

CLINTON

$1422436 \mathrm{N0} 714052.1$ $2422557 N 0714048.1$

$1422434 \mathrm{NO} 714048.1$

$4 \quad 422440 N 0714207$.

272 MDPW

MDPW

VAN BRODE MILL HOPFMAN BROS

CLINTON TOWN

$5422435 N 0714053.1$

$6422436 N_{0} 714046.1$

$7422423 N 0714015$.

8
9 $422441 N 0714021.1$

560

VAN BRODE MILL

1940 W

$1946 \mathrm{C}$

$\overline{--}$

1967

$10 \quad 422517 \mathrm{~N} 0714133.1 \quad 310$

12 422520N0714139.1 298

14 422522N0714141.1 298

$15422520 N 0714134.1300$

16 422530N0714142.1 315

$18 \quad 422525 \mathrm{~N} 0714131.1 \quad 29 \mathrm{~B}$

$19422605 N_{0} 714117.1$

COLONIAL PRESS $1963=$

$1422555 N 0714130$.

$23422603 N 0714119.1265$

$1422354 \mathrm{~N} 0714101.1355$

2422506 N0714112.1 310

$\begin{array}{lll}3 & 422502 N 0714113.1 & 315 \\ 4 & 422447 N 0714110.1 & 375\end{array}$

21 $424027 \mathrm{~N} 0713150.1 \quad 252$ $31423924 N 0713111.1$

$32 \quad 423903 N 0713140.1$

252
205
220

$\begin{array}{lll}33 & 423913 N 0713141.1 & 210 \\ 34 & 423928 N 0713152.1 & 240\end{array}$

$36 \quad 424012 N 0113145.1225$

37 424011N0713148.1 225

$40 \quad 424112 N 0713147.1210$

$44 \quad 424143$ N0713236.1

$45424126 \mathrm{NO} 713250.1$

$46 \quad 424053 N 0713255$.

64 423855NO713203.

$65423856 \mathrm{NO} 713201$.

200

200

279
275
255

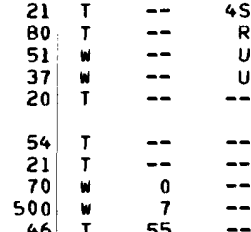

$+16$

$\begin{array}{ll}3-36 & \\ 7-46 & N \\ 7-45 & N\end{array}$

$9-52 \quad H$

$3-62 \quad H$

$\begin{array}{rrr}9 & 5- & H \\ 40 & 11-50 & H \\ 21 & 6-59 & H \\ 18 & 7-60 & H \\ 10 & 12-57 & H\end{array}$

$\begin{array}{rrr}15 & 4-60 & H \\ 25 & 6-67 & H \\ 7 & 12-43 & H \\ 6 & 10-69 & H \\ 20 & 12-64 & H\end{array}$

8
5
3
8
11
10
3
2
5
4
6
20
5
4
5

--
$=-$
$=-$
--
$\overline{--}$
$=-$
--
$=-$
--
--
VAN BRODE MILL

RYLL JOHN

SULLIVAN F J

VAN BRODE MILL

SUPRENANT MFG

SUPRENANT MFG

SUPRENANT MFG

SUPRENANT MFG

GORHAM FRANK

$1964 W$

QUEENEY JAMES

MARINE PLASTICS 1961

CLINTON PLASTIC 1963 C

COLONIAL PRESS 1963 -

COLONIAL PRESS

$1963=$

$1957 B$

CLINTON SR HIGH $1960 \mathrm{~V}$

ST JOHNS CHURCH 1966 V

OUR LADY JASNA

GARDNER JOHN

ENGL ISH W

FEDL LAND BANK

CONNOLLY THOMAS

HOUSE E

TULLY CLARA

MARTIN C V

20

--
$7-48$
$6-66$

$6-66$
$7-67$

$\begin{array}{lll}-- & U & -- \\ 4-61 & N & 250\end{array}$

$\begin{array}{rrr}4-61 & N & 273 \\ 8-63 & N & 302\end{array}$

$\begin{array}{lll}8-63 & N & 302 \\ 9-63 & N & 400\end{array}$

$\begin{array}{lll}97 & 97 & 36 \\ 54 & -- & 3 R\end{array}$

$75 w$

313
1000

65
122

$83 T$

83
185

$148 \mathrm{~T}$

148
49

\begin{tabular}{l|l}
55 & $T$ \\
13 & $T$
\end{tabular}

DUNSTABLE

CMAPMAN E B

TROTMAN GERALO

HUNTER A $\begin{array}{rrrrr}50 & w & - & U & 7 \\ 11 & W & -- & U & 6 \\ 25 & U & -- & U & 21 \\ 22 & U & -- & U & 18 \\ 25 & W & -- & U & 19\end{array}$

$\begin{array}{rrrr}60 & w & -- & -- \\ 10 & u & -- & u \\ 18 & u & -- & u \\ 11 & w & -- & - \\ 25 & w & -- & u\end{array}$

$\begin{array}{rr}16 & w \\ 12 & U\end{array}$

189
413

$\begin{array}{cc}16 & - \\ 109 & U \\ 100 & --\end{array}$

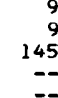

$\begin{array}{rrr}-- & H & -\overline{5} \\ 3-61 & \mathrm{~N} & 15 \\ 1-63 & \mathrm{~N} & 300\end{array}$

$11-63 \mathrm{~N} \quad 1040$

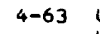

$=-$

$4-63$
$11-57$

3-60

$$
--
$$

$4-39$
$6-39$
$6-39$
$6-39$
$6-39$

$--$

$6-39$
$6-39$

$6-39$
$6-39$

6-39 
LOCAL WULLL LATITUDELONGITUDE
ALTITUOE DWNER OR USER (FT)
YEAR/
METHOD DIAM-IFIN-IOEPTHIUSE TEET WATER-
BEARING DRILLED ETER IISH I BET I BED- MATERIAL

IIN) I I (FT)
DUNSTABLE --CDNTINUED $2423452 N 0714746.1 \quad 438$ $1423538 \mathrm{N0714658.1} 420$ $\begin{array}{lll}2 & 423536 N 0714658.1 & 420 \\ 3 & 423535 N 0714707.1 & 420\end{array}$

$423423 \mathrm{NO} 14637$

$\begin{array}{lll}5 & 423521 N 0714638.1 & 420 \\ 6 & 423519 N 0714641.1 & 400\end{array}$

$7423517 N 0714644.1390$

$8 \quad 423514$ N0714646.1 385

9 423419N0714630.1 362

423417N0714626.1 363

$11423424 N 0714631.1 \quad 358$

$13423442 \mathrm{NO} 714626.1370$

$14 \quad 423437$ N0714626.1 370

$15423404 N 0714631.1$

6423346 N0714626.1

7423650 NO 714752 .

$20423407 N 0714702$.1

$21423609 N 0714716.1 \quad 585$

$\begin{array}{lll}22 & 423319 N 0714509.1 & 380 \\ 23 & 423352 N O 714619.1 & 352\end{array}$

$24 \quad 423344 N 0714636.1$

$25 \quad 423540 N 0714747.1$

26 423532N0714843.1

$27 \quad 423540$ NO714825.1

$29 \quad 423508$ N0714833.1 465

$30 \quad 423453 N 0714628.1 \quad 368$

$31423505 N 0714637.1378$

33 423509NO714643.1 378

34 423315N0714703.1 500

$35 \quad 423317 N 0714657.1500$

36 423324NO714702.1 488

37 423419N0714653.1 390

38423358 No714616.1

39423407 N0714022.1

$40423433 N 0714955.1 \quad 540$

4 423437No714955.1 525

$\begin{array}{lll}42 & 423432 N 0715001.1 & 530 \\ 43 & 423417 N 0714712.1 & 410\end{array}$

$44 \quad 423506 N 0714904.1 \quad 485$

$\begin{array}{lll}45 & 423350 N 0714621.1 & 351\end{array}$

$\begin{array}{lll}46 & 423355 N 0714624.1 & 355 \\ 47 & 423448 N 0714726.1 & 425 \\ 48 & 423333 N 0714614.1 & 365\end{array}$

$49 \quad 423352 N 0714942.1 \quad 755$

50423348 N0714932.1 755

51423703 N0714955.1 968

$\begin{array}{lll}52 & 423614 N 0715049.1 & 960 \\ 53 & 423519 N 0715049.1 & 870\end{array}$

$54 \quad 423758$ N0714745.1 750

55 423644N0715059.1 1092

56 423719N0714707.1 810

$\begin{array}{rrr}57 & 423611 N 0715032.1 & 1010 \\ 58 & 423433 N 0715014.1 & 595\end{array}$

59423347 N0714914.1 758

$\begin{array}{lll}60 & 423328 N 0714954.1 & 848 \\ 61 & 423529 N 0714825.1 & 625\end{array}$

$\begin{array}{lll}61 & 423529 N 0714825.1 & 625 \\ 62 & 423343 N 0114826.1 & 812\end{array}$

63 423711NO715101.1 1150

$64 \quad 423740 N 0715018.11000$

$\begin{array}{lll}423322 N 0714640.1 & 428\end{array}$

$\begin{array}{lll}1 & 423248 N 0714503.1 & 318 \\ 2 & 423244 N 0714506.1 & 322\end{array}$

3423245 NO 114510.1322

$4 \quad 423338$ N0715046.1 589 5 423354NO114610.1 350

$\begin{array}{lll}6 & 423346 N 0714611.1 & 349 \\ 7 & 423336 N 0714606.1 & 345\end{array}$

8423330 NO714601.1 332
LACOMBE HENRY

MDPW

MDPW

FITCHBURG CITY

FITCHBURG CITY
FITCHBURG CITY

FITCHBURG CITY

FITCHBURG CITY

FITCHBURG CITY

FITCHBURG CITY

FI TCHBURG CITY

FITCHBURG CITY

FITCHBURG CITY

FITCHBURG CITY

$1945 V$

FI TCHBURG

FITCHBURG CITY

SIMONDS SAW STL

FALULAH PAPER

KELLL BARTOW
PIERCE ERNEST

$1951 \mathrm{~V}$

$1953 \mathrm{~V}$

WACH POTATO CHP

CHUBBYS MARKET

IISON BERNARD

IILSO

$\begin{array}{ll}\text { PIERMAROCCHI F } & 1967\end{array}$

DUVAL CAMILLE $1960 \mathrm{P}$

$\begin{array}{lll}\text { FDRD MAYNARD } & 1965 & p \\ \text { CRDCKER C T 3RD } & 1966 & p\end{array}$

CRDCKER DDUGLAS $1965=$

HARROWER NORMAN 1965 P

FITCHBURG CITY 1965 P

MATTHEWS MOTORS 1962 P

PLAZA CAR WASH 1965

ST BERNARDS CEM 1966

GERKEY PHDTD

$1969 \mathrm{C}$

KING MITCHELL E 1951 C

FDRREST ANGLINE 1965

$\begin{array}{ll}\text { CHRISTIAN F } & 1963 \\ \text { RUSSO CONST CD } & 1971\end{array}$

AZARIAN PLASTIC 1964

SIMDNDS S + S 1965 C

FITCHBURG PAPER 1952

$\begin{array}{ll}\text { FITCHBURG PAPER } & 1956 \\ \text { FITCHBURG PAPER } & 1956\end{array}$

$\begin{array}{ll}\text { FITCHBURG PAPER } & 1956 \\ \text { GR AMER PLASTIC } & 1956\end{array}$

WATATIC SPIN 1944

$\begin{array}{llll}\text { FALULAH PAPER } & 1964 & \mathrm{C} \\ \text { FALULAH PAPER } & 1968 & \mathrm{C}\end{array}$

FITCHBURG G \& E $1966=$

OAK HILL CC 1968

OAK HILL CC

1968

CASASSA EUGENE 1964

IITA AHTI O 1967

FERGUSDN WM 1946

FAIRBANKS ROBT 1964

$\begin{array}{ll}\text { WEEKS NORMAN } & 1961 \\ \text { ROMAND A J } & --\end{array}$

ROMAND A J

$1964 \bar{P}$

LAMOTHE ALBERT 1965 -

GODIN ROBT A 1965

DEJONGE LOUIS

CDOK R P

$1961 \mathrm{C}$

1957

LEOMINSTER CITY 1950

FITCHBURG CITY 1968

$\begin{array}{ll}\text { FITCHBURG CITY } & 1968 \\ \text { FITCHBURG CITY } & 1968\end{array}$

FITCHBURG CIIY 1968

FITCHBURG CITY 1968

F I TCHBURG CITY

FITCHBURG CITY
209

$\begin{array}{rrrr}14 & T & -- & -- \\ 34 & T & -- & - \\ 21 & T & 23 & B G \\ 45 & T & -- & G \\ 17 & T & 17 & R R \\ 5 & T & -- & -- \\ 34 & T & -- & R \\ 13 & T & -- & 2 S \\ 14 & T & -- & 2 S \\ 19 & T & -- & Q\end{array}$

$\begin{array}{rrrr}60 & T & 66 & 4 G \\ 40 & T & -- & G \\ 50 & T & 5 B & R \\ 60 & T & -- & R \\ 40 & T & -- & --\end{array}$

$\begin{array}{rrrr}40 & T & - & 2 S \\ 60 & W & 60 & R\end{array}$

$\begin{array}{llll}60 & & 60 & R \\ 57 & W & -7 & R\end{array}$

$\begin{array}{rrr}700 & & 7 \\ 175 & w\end{array}$

$460 w$

291

705
46

$61 W$

208 w 46 --

$\begin{array}{rrrr}208 & w & 46 & -- \\ 204 & 2 & -- \\ 265 & 7 & 144 & --\end{array}$

$\begin{array}{rrrr}700 & 144 & -- & 20\end{array}$

$\begin{array}{rrrr}990 & W & 60 & - \\ 130 & W & 65 & - \\ 29 & - & R \\ 46 & W & -- & 4 S\end{array}$

36

74

90
140

$\begin{array}{rr}140 & W \\ 42 & T\end{array}$

14
22
16

$\begin{array}{lll} & T & -5\end{array}$

$44 \mathrm{~W}--\quad 4 \mathrm{G}$

280
55

$\begin{array}{rrrr}71 & T & 79 & =\end{array}$

$\begin{array}{rrrr}59 & T & -- & S \\ 68 & W & 72 & R \\ 62 & W & -- & R \\ 360 & W & 50 & -- \\ 55 & T & 62 & R\end{array}$

$400 \div 70$

400

400
270

$184 W 50 \quad=$

$\begin{array}{lll}27 & -- & 22\end{array}$

172

$\begin{array}{llll}172 & w & 9 & -- \\ 400 & w & 17 & --\end{array}$

$220 w \quad 40 \quad \cdots$

135

895

550
120

$\begin{array}{lll}120 & W & 24 \\ 430 & W\end{array}$

$\begin{array}{rrrr}220 & - & 104 & - \\ 45 & T & -- & 5 \\ 27 & T & -- & 2 S \\ 30 & T & -- & 2 S \\ 20 & T & -- & 25\end{array}$

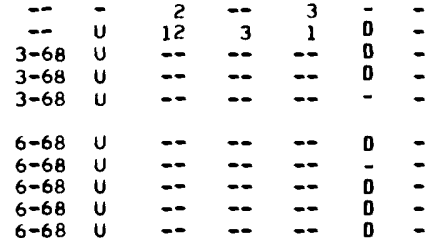


LOCAL

WELL
LATITUDE- $\quad$ ALTI LATITUDE- TUDE
LONGITUDE
OWNER OR USER
YEAR, - WELL METHOD DIAAM-IFIN-IDEPTHIUSE TO BEARING LEVELINATE IUSE ȲIFLDI DD ITIMF LDG OW

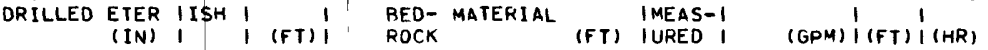

FITCHBURG --CONTINUED
$\begin{array}{lrrr}X & 9 & 423324 N 0714555.1 & 332 \\ \times & 10 & 423311 N 07114538.1 & 338\end{array}$ $\begin{array}{lll}10 & 423311 N 0714538.1 & 338 \\ 11 & 423400 N 0714646.1 & 370\end{array}$
FITCHBURG CITY FITCHBURG CITY
FITCHBURG CITY
$1968 w$
$1968 w$

1968 W

GARUNER

W $76 \quad 423710$ NOT1580S.1 1085

$1423 B 45 N 0713138.1$ 4 423808N0713405.1 9 423905N0713411.1 $10423914 N 0713411.1$

$11423825 \mathrm{NO} 713334.1$ $12423824 N 0713339$. $16 \quad 423756 N 0713848.1$ 18 423859N0713928.1 288

19423851 N0713920.1 285 $\begin{array}{lll}23 & 423546 N 0713323.1 & 330 \\ 24 & 423654 N 0713436.1 & 335\end{array}$ $\begin{array}{lll}25 & 423700 N 0713512.1 & 250 \\ 26 & 423635 N 0713637.1 & 218\end{array}$

27 423658N0713644.1 $28 \quad 423801 N 0713401.1$ $29 \quad 423923 N 0713249.1$ 30423749 N0713721.1

$33423502 N_{0} 713456.1$ $47 \quad 423715 \mathrm{NO} 113834.1$ 49 423752N0713258.

50423630 NO713632.1 51423713 N0713654.1

52 423842N0713913.1 53 423801NO713851.1 54 423553N0713341.

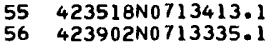

$59 \quad 423837$ N0713346.1

60423830 NO713412.1

$61423808 N 0713408.1$

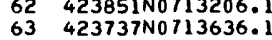

$64 \quad 423736 N 0713635.1$ $65423835 N 0713336.1$ $66423837 N 0713324$. 68 423546NO713620.1

$69423738 \mathrm{~N} 0713504.1$ $70423833 N 0713424.1$

$71423739 \mathrm{~N} 0713445.1$ 72
73 423741 N0713438.1

$74 \quad 423835 N_{0} 713209.1$ $\begin{array}{lll}75 & 423658 N 0713547.1 & 250\end{array}$

235
270
250
235
230
300
300
270
280
288
285
330
335
250
218
250
275
262
320
240
295
265
302
228
290
281
270
325
310
248
275
242
255
278
232
228
270
270
268
211
210
212
225
235
202
220
250

CAMP COLLER 1964 - 6 X

GROTON

FITZPATRICK J GOSSELIN BLDRS MANDERSON M

PRIEST DAVIU $B$

ENGLISH HAROLO ENGLISH GERARD 1966 ROBERTSON N S 1969 WEATHERBEE R N 1960

STERN MILTON

JOHNSON NDRMAN

ONEILL EDW J

WEZLETS MM $M$

HAYES JOSEPH S

HOLT JOHN E

NORRIS DAVIO L

$\begin{array}{ll}1961 & P \\ 1951 & C\end{array}$

1967
1949

1958

DOLIVE JANET

1962

WHITE DOUGLAS

1965

BERTDZZI RALPH

LIVINGSTONE MEL

FLOYD HARRY

BRITT M C

BUSCEM

COUNTRY CLUB

$\begin{array}{lll}\text { RENNER MAYNARD } & -\overline{-} & - \\ \text { FORWARD LEONARO } & 1973 & \mathrm{C}\end{array}$

NAYLOR EDWIN L $1964 \mathrm{~V}$

MILLER CHARLES 1965

HANNEMANN PETER 1969

$\begin{array}{ll}\text { BELL LESLIE G } & 1964 \\ \text { GREENHOW PHILIP } & 1973\end{array}$

ANDREASSEN

GROTON TOWN

GROTON TOWN

GROTON TOWN

1973

GROTON TOWN

1963

1963

GROTON TOWN

GROTON TOWN

GROTON TOWN

GROTON TOWN

GROTON TOWN

1663

GROTON TOWN

GROTON TOWN

1963

1963

1963

1963

1963
1966

\begin{tabular}{|c|c|c|c|}
\hline 36 & $w$ & 26 & $w$ \\
\hline 6 & $x$ & 251 & $\omega$ \\
\hline 6 & $x$ & 203 & $w$ \\
\hline 6 & $x$ & 188 & $w$ \\
\hline 6 & $x$ & 320 & $w$ \\
\hline 6 & $x$ & 530 & $w$ \\
\hline 6 & $x$ & 100 & $W$ \\
\hline 6 & $x$ & 310 & $w$ \\
\hline 4 & $x$ & 26 & $w$ \\
\hline 6 & $x$ & 220 & $w$ \\
\hline-- & - & 125 & $w$ \\
\hline 6 & $x$ & 160 & $w$ \\
\hline 6 & $x$ & 106 & $w$ \\
\hline 6 & $x$ & 380 & $w$ \\
\hline 6 & $x$ & 70 & $w$ \\
\hline 6 & $x$ & 230 & $w$ \\
\hline 6 & $x$ & 140 & $w$ \\
\hline 6 & $x$ & 245 & $w$ \\
\hline 6 & $x$ & 185 & $w$ \\
\hline 6 & $x$ & 100 & $w$ \\
\hline 6 & $x$ & 143 & $w$ \\
\hline 6 & $x$ & 101 & $w$ \\
\hline 6 & $x$ & 58 & $w$ \\
\hline 6 & $x$ & 174 & $w$ \\
\hline 6 & $x$ & 350 & $w$ \\
\hline 2 & $\mathbf{P}$ & 28 & $w$ \\
\hline 6 & $x$ & 80 & $w$ \\
\hline 6 & $x$ & 200 & $w$ \\
\hline-- & $=$ & 80 & $w$ \\
\hline 6 & $x$ & 275 & $w$ \\
\hline 2 & $T$ & 20 & $w$ \\
\hline 6 & $x$ & 135 & $w$ \\
\hline 2 & $\mathbf{T}$ & 26 & $w$ \\
\hline 2 & S & B5 & $w$ \\
\hline-- & - & 500 & $w$ \\
\hline-- & $x$ & 380 & $w$ \\
\hline 2 & 0 & 37 & $T$ \\
\hline 2 & 0 & 26 & $T$ \\
\hline 2 & 0 & 26 & $T$ \\
\hline 2 & 0 & 66 & $T$ \\
\hline 2 & 0 & 22 & $\pi$ \\
\hline 2 & 0 & 25 & $\mathbf{T}$ \\
\hline 2 & 0 & 18 & $T$ \\
\hline 2 & 0 & 18 & $T$ \\
\hline 2 & - & 54 & $T$ \\
\hline 2 & 0 & 35 & $T$ \\
\hline 2 & 0 & 62 & $T$ \\
\hline \multicolumn{4}{|c|}{ HARVARO } \\
\hline$\ldots$ & - & 20 & $T$ \\
\hline$\ldots$ & 0 & 92 & $T$ \\
\hline 1 & - & 28 & $T$ \\
\hline$i$ & - & 40 & $T$ \\
\hline 1 & - & 29 & $T$ \\
\hline 1 & - & 26 & $T$ \\
\hline 1 & - & 58 & $T$ \\
\hline 1 & - & 108 & $\mathbf{T}$ \\
\hline 1 & - & B2 & $T$ \\
\hline 1 & - & 31 & $\mathbf{T}$ \\
\hline 6 & $x$ & 88 & $w$ \\
\hline 6 & $x$ & 80 & $w$ \\
\hline 6 & $x$ & 147 & $w$ \\
\hline 6 & $x$ & 133 & $w$ \\
\hline 6 & $x$ & 83 & $w$ \\
\hline 6 & $x$ & 175 & $w$ \\
\hline 6 & $x$ & 64 & $w$ \\
\hline 6 & $x$ & 69 & $w$ \\
\hline 6 & $x$ & 129 & $w$ \\
\hline 6 & $x$ & 68 & $w$ \\
\hline
\end{tabular}

$9 \quad T \quad--\quad 4 R$

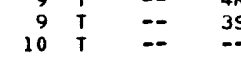

$\begin{array}{llllllll}7 & 6-68 & U & -- & -- & -- & 0 & - \\ 7 & 6-68 & U & -- & -- & -- & 0 & - \\ 0 & 6-68 & U & -- & -- & -- & 0 & -\end{array}$

$\begin{array}{lllllllll}16 & -64 & H & 25 & - & - & - & \end{array}$

1 423210 N0713448.1

1
2 $423214 N 0113542.1$

$\begin{array}{ll}3 & 423307 N 0713714.1 \\ 5 & 423122 N 0713425.1\end{array}$

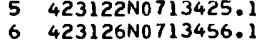

234
255
226

MDPW

MDPW

MOPW

$7423120 N 0713404.1 \quad 307 \quad M D P W$

$10423114 \mathrm{NO} 713800$.

11 $423102 N 0713633.1$

$12423101 \mathrm{~N} 0713626.1$

218
265
275

$4 \quad 422842 \mathrm{NO} 713512.1$ 422830 NOT13521.

10 422911N0713507.1

$11422912 N 0713507$. 422917 N0713459.1

567

582
565

$14 \quad 422755 N_{0} 713603.1$ 570

MDPW

MDPW

MDPW

MORSE C B MO

SMITH CLARENCE

KROPP GEO V

NEWMAN C A

GROSS ERVIN JR

KLEIN J LESTER

LAHANAS C J

HARVARO

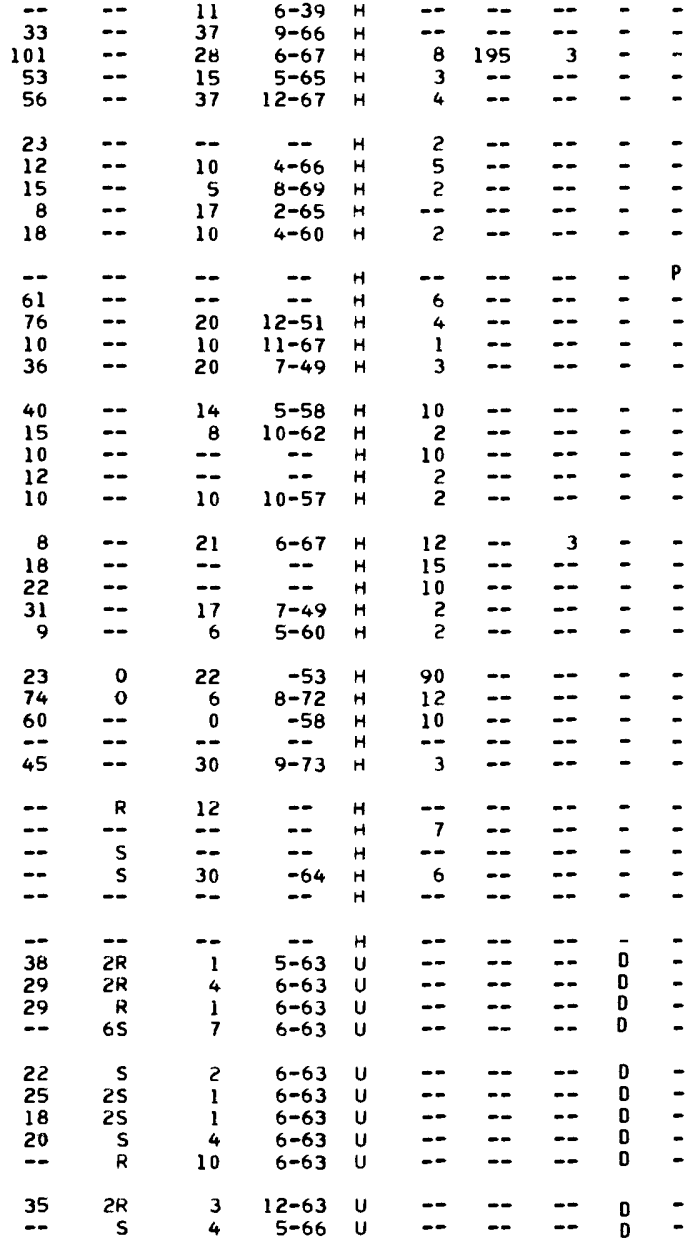

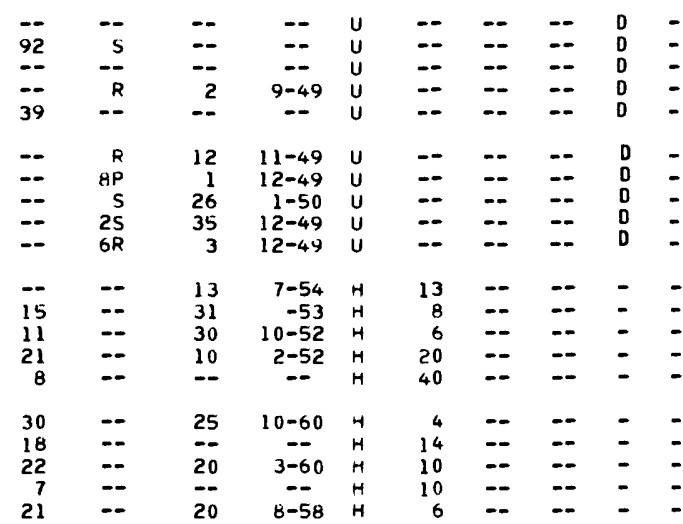


TABLE 1.--DESCRIPTION OF SELECTED WELLS, TEST WELLS, AND BORINGS -- CDNTINUED

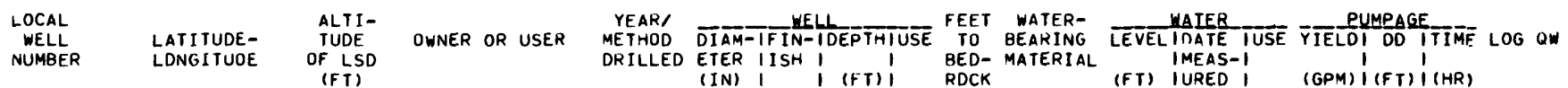
HARVARD - -CONTINUED

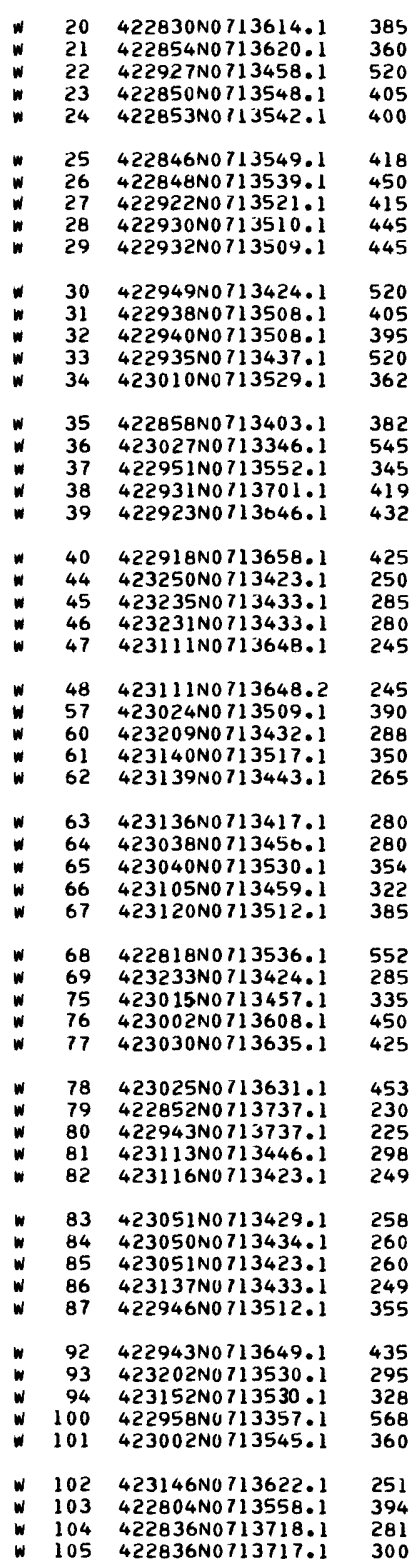

W 105 422836N0713717.1 300

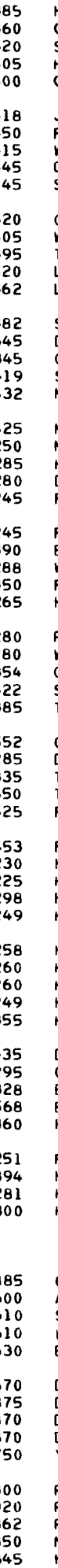

\begin{tabular}{|c|c|}
\hline $\begin{array}{l}\text { HEINZ GOEHRING } \\
\text { GATES KENNETH M } \\
\text { STRONG R A } \\
\text { HOSIE JAMES } \\
\text { GRAHAM JDHN S }\end{array}$ & $\begin{array}{l}1955 \\
1971 \\
1953 \\
1964 \\
1962\end{array}$ \\
\hline $\begin{array}{l}\text { JOHANSSON EOW } \\
\text { FARLEY EDWARD } \\
\text { WETHERBEE RE } \\
\text { OSBDURNE A } \\
\text { STRANG DONALD B }\end{array}$ & $\begin{array}{l}1960 \\
1955 \\
1957 \\
1957 \\
1955\end{array}$ \\
\hline
\end{tabular}

STRANG DONALD B 1955 P

GDRDON HERBERT $1965 \mathrm{P}$

WELLS IRVING

TITUS SIONEY

LEE JOHN
LOZIER F

$\begin{array}{ll}1965 & p \\ 1961 & p \\ 1961 & p \\ 1957 & p \\ 1960 & p\end{array}$

SMITH FRANKLIN

DUNLAP JAMES A 1958 P

$\begin{array}{lll}\text { GREEN EYRIE CMP } & 1963 & P \\ \text { SAWYER HELEN } & 1964 & \text { P }\end{array}$

$\begin{array}{ll}\text { SAWYER HELEN } & 1964 \\ \text { MAXANT THED W } & 1970 \text { P }\end{array}$

MAXANT WM T $1970 \mathrm{P}$

MAXANT WM T $1949 \mathrm{C}$

HAWBUCK DRCHARD 1963 P

DOE WM G

1958
1941

FDRT DEVENS

$\begin{array}{lll}\text { FDRT DEVENS } & 1940 & \text { W } \\ \text { BURDICK } & 1949 & \mathrm{C} \\ \text { WILKY DIANNE E } & 1970 & \mathrm{P}\end{array}$

FOUNTAIN HUGH G 1959

HARVARD COM CTR 1949 C

REEHL WILLIAM

WDOD MENRY $S$

$\begin{array}{lll} & 1964 & P \\ \text { SISTD JOHN } & 1963 & p\end{array}$

TROY SHERMAN P 1960 P

CALLAHAN J

DOE ORLANDO

THOMAS GEORGE

TUF TS LANA

FRUITLANDS MUS

$1952 P$

FRUITLANDS MUS

HARVARD TOWN

HARVARD TDWN

HARVARD TDWN

HARVARD TDWN

\begin{tabular}{|c|c|c|c|}
\hline 6 & $x$ & 96 & $w$ \\
\hline 6 & $x$ & 245 & $w$ \\
\hline 6 & $x$ & 89 & $w$ \\
\hline 6 & $x$ & 102 & - \\
\hline 6 & $x$ & 95 & $w$ \\
\hline 6 & $x$ & 420 & $w$ \\
\hline 6 & $x$ & 155 & $w$ \\
\hline 6 & $x$ & 130 & $w$ \\
\hline 6 & $x$ & 110 & $w$ \\
\hline 6 & $x$ & 105 & $w$ \\
\hline 6 & $x$ & 115 & $w$ \\
\hline 6 & $x$ & 85 & $w$ \\
\hline 6 & $x$ & 108 & w \\
\hline 6 & $x$ & 110 & 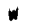 \\
\hline 6 & $x$ & 42 & $w$ \\
\hline 6 & $x$ & 100 & $w$ \\
\hline 6 & $x$ & 175 & $w$ \\
\hline 6 & $x$ & 150 & $w$ \\
\hline 6 & $x$ & 200 & $w$ \\
\hline 6 & $x$ & 195 & $w$ \\
\hline 6 & $x$ & 185 & $w$ \\
\hline 6 & $x$ & 140 & $w$ \\
\hline 6 & $x$ & 500 & $w$ \\
\hline 6 & $x$ & 100 & $w$ \\
\hline 24 & G & 76 & $w$ \\
\hline 2 & S & 76 & 0 \\
\hline 6 & $x$ & 126 & $w$ \\
\hline 6 & $x$ & 100 & $w$ \\
\hline 6 & $x$ & 72 & $\mathbf{w}$ \\
\hline 6 & $x$ & 134 & $w$ \\
\hline 6 & $x$ & 130 & $w$ \\
\hline 6 & $x$ & 90 & $w$ \\
\hline 6 & $x$ & 65 & $w$ \\
\hline 6 & $x$ & 170 & $w$ \\
\hline 6 & $x$ & 175 & $w$ \\
\hline 6 & $x$ & 81 & 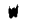 \\
\hline 6 & $x$ & 98 & $\mathbf{W}$ \\
\hline 6 & $x$ & 50 & $w$ \\
\hline 6 & $x$ & 70 & $w$ \\
\hline 6 & $x$ & 350 & $T$ \\
\hline 6 & $x$ & 275 & $w$ \\
\hline 2 & 0 & 48 & $T$ \\
\hline 2 & 0 & 19 & $T$ \\
\hline 2 & D & 33 & $T$ \\
\hline 2 & 0 & 50 & $T$ \\
\hline 2 & 0 & 49 & $T$ \\
\hline 2 & 0 & 5 & $T$ \\
\hline 2 & D & 27 & $T$ \\
\hline 2 & D & 53 & $T$ \\
\hline 8 & $x$ & -- & $T$ \\
\hline 6 & $x$ & 186 & $w$ \\
\hline 6 & $x$ & 200 & $w$ \\
\hline 6 & $x$ & 350 & $w$ \\
\hline 6 & $x$ & 124 & $w$ \\
\hline - & - & 95 & $w$ \\
\hline 42 & - & 64 & $w$ \\
\hline 0 & $x$ & 110 & $w$ \\
\hline 6 & $x$ & 100 & $w$ \\
\hline 6 & $x$ & 250 & $w$ \\
\hline
\end{tabular}

$\begin{array}{rr}110 & = \\ 10 & = \\ 19 & = \\ 14 & \\ 8 & = \\ 30 & = \\ 2 & = \\ 9 & = \\ 14 & \\ 14 & \\ 28 & - \\ 34 & \\ 60 & \\ 6 & \end{array}$

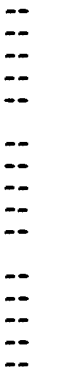

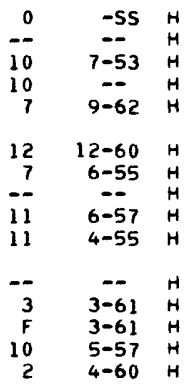

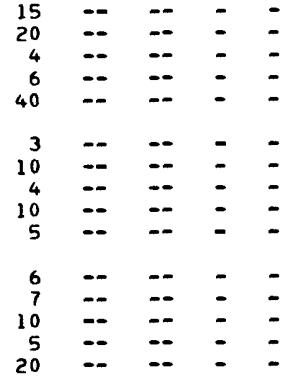

$\begin{array}{rrrrr}7 & -- & -- & -- & H \\ 2 & -- & 19 & 9-58 & H \\ 4 & -- & 16 & 11-63 & P \\ 82 & -- & 20 & 12-64 & H \\ 92 & -- & 60 & 10-70 & H\end{array}$

$41 \quad-$

30

$2-70 \quad H$

30
+1
10
12
10

$\begin{array}{ll}2-70 & H \\ 3-49 & H \\ 5-63 & - \\ 7-58 & H \\ 1-41 & P\end{array}$

$3 \quad 12-40 \quad U$

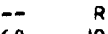

$\begin{array}{rr}60 & 10 \\ 61 & -- \\ 1 & --\end{array}$

$\overline{--}$

$=-$
$=-4$

$\begin{aligned} 23 & - \\ 44 & =- \\ 54 & =- \\ 3 & --\end{aligned}$

20
2
40
--
20

$10-66 \mathrm{C}$

$\begin{array}{rr}8-61 & H \\ 12-64 & H\end{array}$

-50
-6

$\begin{array}{ll}24 & =- \\ 38 & =-\end{array}$

20
21
-2
20

$0-52$
$6-54$

$9-54$

$9-69$

$\begin{array}{rrr}26 & -- & 3 \\ 47 & T & 4 \\ 19 & -- & - \\ 33 & -1 & 2 \\ 51 & -- & 2\end{array}$

$\begin{array}{ll}4-48 & H \\ 8-71 & U \\ --7 & U \\ 7-71 & U \\ 7-71 & U\end{array}$

\begin{tabular}{c}
9 \\
\hdashline- \\
--
\end{tabular}

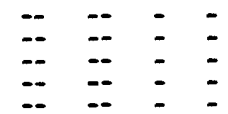

HAKVARD TOWN

HARVARD TOWN

HARVARD TDWN

MARVARD TDWN

HAKVARD TOWN

1971

1971

1971

1971
1953

DAY DORIS $M$

COX ELIZABETH

BISSDN RAYMONO

BUNCH JERRY

REEDY JOHN H

$1960 \mathrm{C}$

$1970 \mathrm{~A}$

1970

1966

FDRT DEVENS

1952 -

HARRIS REV JOHN 1955

HARRIS REV JOHN
HOLDEN

CONDON CO 1949

SKANTZ CONRAD

WELCH JAMES E

GRODEUR HECTOR

1949 C

$8 x$

$100 w$

DOMENITIS TONY 1942

DDURDEVILLE T

DOURDEVILLE

DOURDEVILLE
YOUNG HARDLD

--
--

PATRIDGE WARREN 1939

PAPALIA PHILIP

NIELSON A J

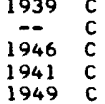

PROUTY RICHARU
97
53

$\begin{array}{rrr}-- & 4 \\ - & -2\end{array}$

$\begin{array}{cc}7-71 & u \\ -- & u\end{array}$

$\begin{array}{ll}7-71 & U \\ 9-71 & U\end{array}$

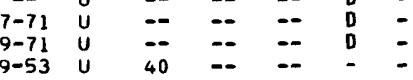

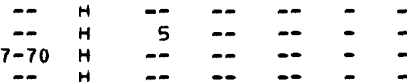

$\begin{array}{rrrrrr}-- & P & 1050 & -- & - & = \\ -55 & H & 22 & -- & - & = \\ - & H & -- & -- & -- & -\end{array}$

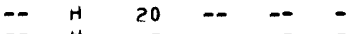




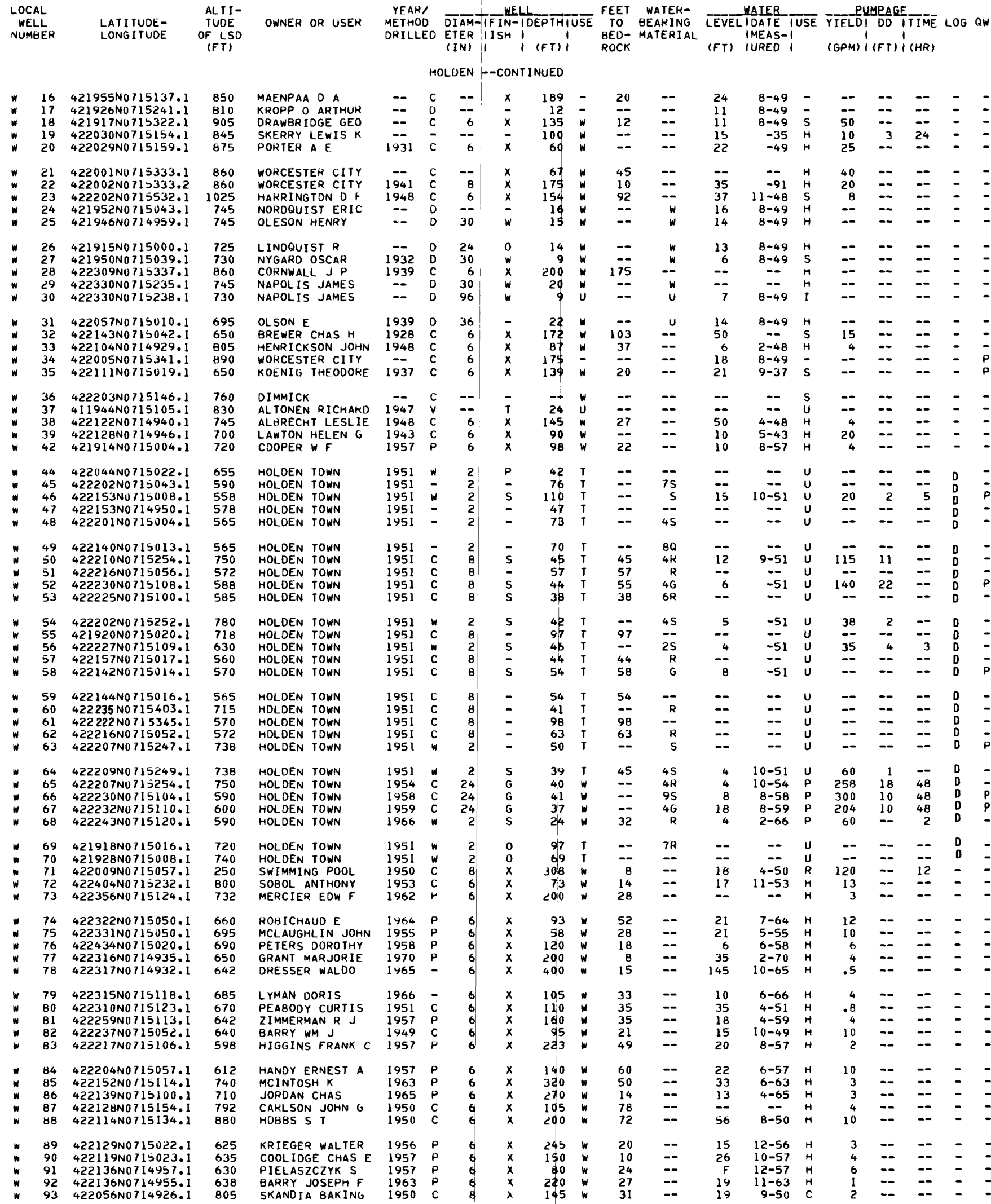


LOCAL

NULLL

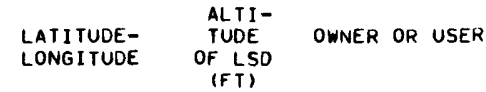

YEAR/
METHOD DIAM-IFIN-IDEFTHIUSE TO BET WATER-
BEARING

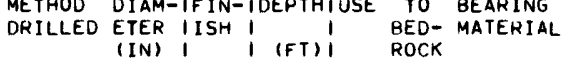
(IN) I I (FT)

$\begin{array}{llll} & 94 & 422049 N 0714956.1 & 752 \\ 95 & 422037 N 0714949.1 & 782 \\ & 96 & 422041 N 0714837.1 & 680 \\ w & 97 & 422029 N 0714854.1 & 772 \\ w & 98 & 422034 N 0714854.1 & 778\end{array}$

$98422034 \mathrm{NO} 14854.12778$

$100422014 N 0715031.1$

* 101 422046NOT15039.1

102422048 NOT15038.1

103 422010NOTIS126.1 761

W $104 \quad 422006 N 0715131.1780$

$\begin{array}{lll}\text { w } 105 & 421948 N 0715154.1 & 860 \\ 106 & 421806 N 0715152.1 & 780\end{array}$

W 107 422133N0715411.1 955

W 108 422053N0715332.1 842

w $109422027 N 0715240.1930$

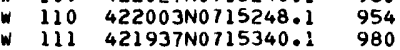
$\begin{array}{lll}w 12 & 421941 N 0715337.1 & 982 \\ & \end{array}$

w $114 \quad 421926$ N071S241.2 810 W 115 422302N0715248.1 738 $116 \quad 422459 N 0715052.1$ 117 422320N0715144.1

W 119422341 N0715108.1 W 120 422211N0715309.1 * 121 422142N0715005. $122422131 N 0714910$. $123422127 N 0714918.1$

w $124 \quad 422103$ N0712306.1 820 $\begin{array}{lll}w 125 & 422316 N 0715054.1 & 672 \\ 126 & 422328 N 0715121.1 & 715\end{array}$ $\begin{array}{lll}126 & 422328 N 0715121.1 & 715 \\ 127 & 422438 N 0715055.1 & 688\end{array}$ W 128 422317N0714955.1 655

* 129 422219N0715118.1 662 $\begin{array}{lll}W 130 & 422316 N 0714943.1 & 606 \\ W 131 & 422231 N 0714920.1 & 580\end{array}$ w 132 422234N0714919.1 595

W $133422355 N 0715057.1$

w $134 \quad 422401 N 0715057.2$ W $135422326 N 0715041.1$

w $136 \quad 422300 N_{0} 715017.1$

w $137422200 N 0715031.1$

W 138 422029N0715009.1

* $139422035 N 0715012.1655$ $\begin{array}{lll}140 & 422042 N 0715019.1 & 648 \\ 141 & 422217 N 0715336.1 & 785\end{array}$

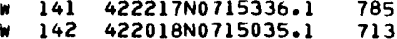
W 143 422310NOT15209.1 638

$\begin{array}{lll}144 & 422314 N 0715212.1 & 670 \\ 145 & 422220 N 071523 B .1 & 730\end{array}$ $\begin{array}{lll}145 & 422220 N 0715238.1 & 730 \\ 146 & 422216 N 0715246.1 & 738\end{array}$ $147 \quad 422219 N 0715245.1$
$148 \quad 422218 N 0715249.1$

w $149 \quad 422222 N 0715253.1750$ w 150 422214N0715250.1 735 isl 422212N0715216.1 $152422207 N 0715254.1$
$153 \quad 422303 N 0715329.1$

W $154 \quad 422303$ N0715132.1 605 $\begin{array}{rrr}155 & 422221 N 0715105.1 & 580 \\ 156 & 422227 N 0715109.2 & 575\end{array}$

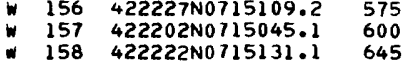

W $159422227 N 0715130.1650$ W $160 \quad 422259 N 0715001.15510$ $\begin{array}{lll}161 & 422303 N 0714959.1 & 510 \\ 162 & 422230 N 0714954.1 & 535\end{array}$ W 163422227 N0715050.1 570

W 164 422130N0715402.1 885 W $165422115 \mathrm{~N} 0715020.1 \quad 643$ $\begin{array}{lll}166 & 422043 N 0714840.1 & 675 \\ 167 & 422253 N 0715019.1 & 563\end{array}$ 168 422258N0715223.1 723 $\begin{array}{llllll}\text { DESROCHES C R } & 1952 & C & 6 & x & 160 \\ \text { WASKIEWICZ F } & 1952 & C & 6 & X & 160\end{array}$

$\begin{array}{llllllll}\text { KIMBALL THOMAS } & 1963 & \mathrm{P} & 6 & \mathrm{x} & 102 & 30 \\ \text { ZOKOWSKI EDWAKD } & 1963 & - & 6 & \mathrm{x} & 73 & 34 \\ \text { BOMBA CHAS } & 1952 & \mathrm{p} & 6 & \mathrm{x} & 88 & 31\end{array}$

OLSON MFG CO

ALMSTROM HOWARD

AINNO CLARENCE 1957 P

LACROIX GEO L

PERRY ROGEH

MCGRAIL WM H

RICHARDS $H G$

MOROSKI EDWARD

FOLEY THOMAS

$\begin{array}{lll}\text { FOLEY THOMAS } & 1968 & \\ \text { STARBARD K A } & 1952 & C \\ \text { WHITE GEORGE A } & 1953 & C \\ \text { REED }\end{array}$

$\begin{array}{lll}\text { REED A B } & 1959 & P \\ \text { MASSEY ROBT K } & 1961 & P\end{array}$

LEMAY JOSEPH E

WARMER GOROON

OUGAN DAVID

GARDNER INAS

CRYSTOFF W

LINDQUIST USCAR 1950

GARDNER WARREN

MARSHAL $C$

HINE STANLEY $R$

BLAKE W 0

KEATING ROBERT

CARVER EVERE

MASON ORRIN

FORSBERG E $\bar{F}$ JR

FORSBERG E $F$

HOLDEN TOWN

HOLDEN TOWN

HOLDEN TOWN

HOLDEN TOWN

HOLOEN TOWN

HOLDEN TOWN

HOLDEN TOWN
HOLDEN TOWN

HOLOEN TOWN

HOLDEN TOWN

HOLOEN TOWN

HOLOEN TOWN
HOLDEN TOWN

HOLOEN TOWN

HOLOEN TOWN

HOLDEN TOWN

HOLOEN TOWN

HOLOEN TOWN

HOLOEN TOWN

HOLOEN TOWN

HOLOEN TOWN

HOLDEN TOWN

HOLDEN TOWN

HOLDEN TOWN

HOLOEN TOWN

HOLDEN TOWN

HOLDEN TOWN

HOLDEN TOWN

HOLDEN TOWN

HOLDEN TOWN

HOLDEN TOWN

LARSON ROLF L

HEINOLO THOMAS

PARKER C R

PARKER
MOC

$1966 w 2$
$1969 \mathrm{C}$

1971

1958

1971

1955

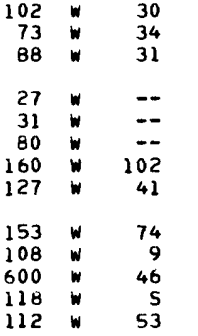

-

$125 w 11$

125
217
137

$104 \%$

$100 \mathrm{~W} 20$

125

100

295
90

68

$217 w 12$

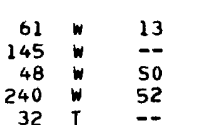

28

20

21
44

28

$1965 w$

$1965 W$

1965

$1966 W$

1966

1966

$\begin{array}{lll}1966 & 2 & 0 \\ 1966 & 2 & 0\end{array}$

1966

1953

1953

1953

$1953 w$

$1953 \mathrm{~V}$

1953

$\begin{array}{lll}1953 & 2 & 0 \\ 1953 & 2 & 0\end{array}$

48

$\begin{array}{ll}50 & 1 \\ 40 & 1 \\ 18 & \end{array}$

$41 \quad 6 \mathrm{R}$

21 I $=--=0$

$\begin{array}{lll}19 & 1 & - \\ 38 & T & 38\end{array}$

$\begin{array}{lll}35 & \mathrm{~T} & 38 \\ 48 & \mathrm{~T} & --\end{array}$

$44 T$

58

38

$\begin{array}{ll}55 & 1 \\ 41 & 1\end{array}$

1953

1953

1953

1953

1953 w

1953

1953

1953

$1962 R$

1965 C

1927
1927

22

50

48

43
37

49
24

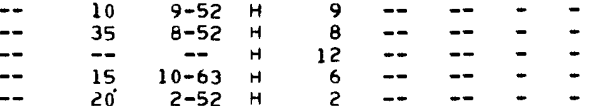

$\begin{array}{lllllllll}4 \mathrm{G} & 2 & 7-69 & \mathrm{~N} & 122 & 14 & 24 & - & -\end{array}$

$\begin{array}{rrrrrrrrr}U & 64 & 8-51 & H & 2 & -- & -- & - & - \\ -- & 55 & 7-57 & H & 10 & -- & -- & - & - \\ -- & 15 & 5-52 & H & 12 & -- & -- & - & -\end{array}$

$\begin{array}{rrrrrrr}20 & 11-68 & H & -- & -- & -- & - \\ 33 & 2-58 & H & 4 & -- & - & -\end{array}$

$\begin{array}{rrrrrrr}2-58 & H & 4 & - & -- & - & - \\ -- & H & 20 & -- & -- & - & - \\ 2-53 & H & 5 & -- & -- & - & - \\ -- & H & 8 & -- & 4 & - & -\end{array}$

$\begin{array}{rrrrrrrl}11 & 12-68 & U & .5 & =- & = & = & - \\ 22 & 2-52 & H & 2 & = & = & = & = \\ 45 & 4-53 & H & 15 & =- & = & = & - \\ 50 & 8-59 & H & 20 & =- & = & = & = \\ 44 & 6-61 & H & 12 & =- & -- & - & -\end{array}$

$\begin{array}{lllllll}-- & H & 5 & -- & -- & - & - \\ -70 & H & 4 & -- & -- & = & - \\ -- & H & 11 & -- & -- & = & -\end{array}$

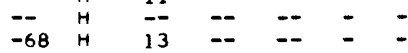

$\begin{array}{lllllll}-- & H & -- & -- & -- & - & -\end{array}$

$\begin{array}{cccccc}--72 & H & 10 & =- & =- & \overline{-} \\ 0-7 & H & 20 & =- & -- & =\end{array}$

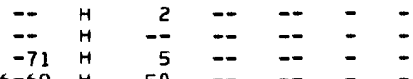

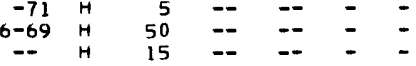

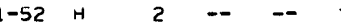

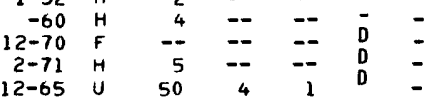

$\begin{array}{cccccc}2-65 & U & -- & -- & -- & 0 \\ -- & U & -- & -- & -- & 0 \\ -- & U & -- & -- & -- & 0 \\ 1-66 & U & 20 & -- & -- & 0 \\ 1-66 & U & -- & -- & -- & 0\end{array}$

$\begin{array}{llllll}1-66 & U & -- & -- & -- & 0 \\ 1-66 & U & 25 & -- & 15 & 0\end{array}$

$\begin{array}{ccccccc}1-66 & U & -- & -- & -- & 0 & - \\ -- & U & -- & -- & -- & 0 & - \\ -- & U & -- & -- & -- & 0 & -\end{array}$

$\begin{array}{llllllll}-- & -- & -- & U & -- & -- & -- & 0\end{array}$

$\begin{array}{rrcr}5 & -- & -- & u \\ 95 & -- & -- & u \\ 5 & 4 & 11-53 & u\end{array}$

$\begin{array}{llll}5 & 16 & 10-53 & U\end{array}$

--

-

$11-53$
$11-53$

$11-53$
$11-53$

$11-53 u$

$11-53$

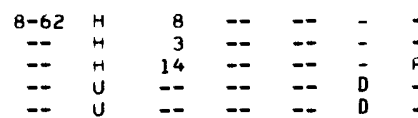


LOCAL

ALTI NUMBE
LATITUDELONGITUDE
ALTITUDE
OWNER OR USER
YEAR' METHOD OIAM-IFIN-IDEPTHIUSE TO BEARING
DRILLED ETER IISH (IN) ROCK

HOLDEN --CONTINUED $\begin{array}{llll}x & 1 & 422340 \mathrm{~N} 0715251.1 & 730 \\ x & 2 & 422340 \mathrm{NO} 715258.1 & 720\end{array}$ $\begin{array}{lll}2 & 422340 N 0715258.1 & 720 \\ 3 & 422340 N 0715304.1 & 720\end{array}$

$\begin{array}{lll}1 & 423114 N 0713802.1 & 219 \\ 2 & 423116 N 0713810.1 & 230\end{array}$

$423111 N 0714135.1 \quad 369$

$422651 N 0714010.1234$

$242273 B N 0714138.1 \quad 375$

3 422803N0714116.1 290

S 422854 N0714107.1 250

$422703 \mathrm{~N} 0714224.1$

$7422711 N 0714139.1 \quad 355$

$\begin{array}{lll}8 & 422519 N 0714254.1 & 340 \\ 9 & 422511 N 0714254.1 & 335\end{array}$

$10 \quad 422506 \mathrm{NO} 0714254.1 \quad 330$

$11422502 \mathrm{NO} 714254.1330$

$12 \quad 422508 \mathrm{~N} 0714253.1340$

$13422502 N 0714252.1 \quad 340$

15 422500NO714253.1 335

17422825 N0714058.1 240

$18422619 \mathrm{NO} 713944.1 \quad 246$

$\begin{array}{lll}19 & 422620 N 0713943.1 & 245 \\ 20 & 423117 . N 0114207.1 & 415\end{array}$

$21423117 \mathrm{~N} 0714207.2 \quad 415$

22423113 N0714137.1 368

$23 \quad 423051$ N0714122.1 355

$\begin{array}{lll}24 & 423054 N 0714255.1 & 405 \\ 25 & 423054 N 0714252.1 & 408\end{array}$

* 26 423114N0714236.1 422

$27 \quad 423144 N 0714130.1395$

$\begin{array}{lll}28 & 423126 \text { N0714102.1 } & 355 \\ 29 & 423121 N 0714056.1 & 345\end{array}$

30 423115N0714108.1 365

31423137 N0714001.1 385

32423057 No $114125.1 \quad 355$

33 423056N0714112.1 360

$34 \quad 423048 N 0714105.1 \quad 354$

$\begin{array}{lll}35 & 423036 N 0714056.1 & 400 \\ 36 & 422738 N 0714300.1 & 492\end{array}$

37 422659N0714215.1 445

$38 \quad 422636 \mathrm{~N} 0714110.1 \quad 295$

$\begin{array}{lll}39 & 422619 \mathrm{~N} 0714056.1 & 235 \\ 40 & 422808 \mathrm{NO} 713913.1 & 245\end{array}$

$\begin{array}{lll}41 & 422817 N 0714057.1 & 238\end{array}$

$42422632 \mathrm{~N} 0714140.1 \quad 275$

43 422609N0714128.1 265

45 422611N0714135.1 268

46 422610NO114146.1 278

$47 \quad 422608 \mathrm{~N} 0714153.1280$

$48 \quad 422609 \mathrm{~N} 0714149.1 \quad 283$

$\begin{array}{lll}49 & 422613 \mathrm{~N} 0714049.1 & 265 \\ 50 & 422627 \mathrm{~N} 0713949.1 & 238\end{array}$

$51422607 N 0713946.1$

$52 \quad 422618$ NOT13932.1 264

53422613 N0713950.1 260

$54422623 \mathrm{NO} 713946.1$

$55423115 \mathrm{~N} 0713830.1$

56 422924NOI14257.1 271

57 422934N0714301.1

58422928 N0714248.1

$59422927 N 0714242.1$

$\begin{array}{lll}60 & 422937 N 0714315.1 & 275 \\ 61 & 422938 N 0714308.1 & 271\end{array}$

272

271

* $62423054 N 0713956.1415$

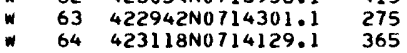

WORCESTER CITY WORCESTER CITY

1930

$1930=-=-$

\section{LANCASTER}

MOPW

MDPW

MDPW

PREST JUDGE W

BAYARD

DEXTER WILLIAM

DEXTER WILLIAM

THCANN ICE CRM

WILLIAM

LANCASTER TOWN

LANCASTER TOWN

LANCASTER TOWN
LANCASTER TOWN

LANCASTER TOWN

LANCASTER TOWN

LANCASTER TDWN
LANCASTER TOWN

LANCASTER TDWN

LANCASTER TOWN

LANCASTER TOWN

HOWARD JDHNSON

HOWARD JOHNSON

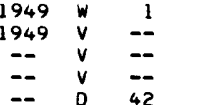

-- 030

$\begin{array}{lll}=- & 0 & 30 \\ =- & 0 & 36 \\ -- & 0 & 36\end{array}$

--

1935

1935

1935

1935
1935

1935

1934

BOY SCOUTS

HOWE ROBERT

ELMO EOWARD

MDPW MAINT BLOG

1934

1962

1968

1954
$1954 \mathrm{C}$

$1970 \mathrm{~A}$

BLANCHETTE

WINTHROP JACK

DAIGLE ANTONIO

BROWN DAVIO

GOULU MORRIS

HUNTER DONALD

DOYLE COTTAGE

TYLER ROBT

FABIAN N W

ATLANTIC UNION

BARMAR PROOUCT

LANCASTER TOWN

LANCASTER TOWN

LANCASTER TOWN

LANCASTER TDWN

LANCASTER TOWN

LANCASTER TOWN

LANCASTER TOWN

LANCASTER TOWN

LANCASTER TOWN

LANCASTER TDWN

LANCASTER TOWN 1960

LEOMINSTER CITY I963

LEOMINSTER CITY 1963

LEOMINSTER CITY $1963=$

LEOMINSTER CITY $1963=$

LEOMINSTER CITY 1963

GLEEZEN KENT 1971 R

$\begin{array}{lll}\text { LEOMINSTER CITY } & 1963 & \text { W } \\ \text { SPARL ING SEDA } & 1974 & -\end{array}$

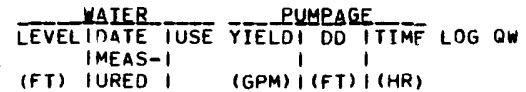
(GPM) I (FT) I(HR) 
LOCAL

WELL

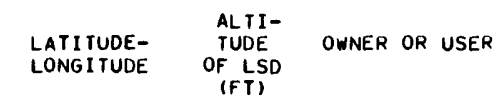

METHOD DIAM-IFIN-IDEPTHIUSE TO WET WATER-

METHOD DIAM-IFIN-IDEPTHIUSE TO BEARING
DRILLED ETER IISH:
(IN) I I (FT)!

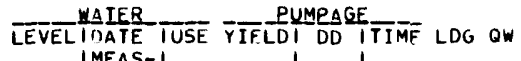
ETER IISH I
(IN) I I
LEOMINSTER

$\begin{array}{ll}423310 N 0714557.1 & 387 \\ 423235 N 0714510.1 & 364\end{array}$ $423213 N 0714443.1347$ 423036NO714331.1 340

$3423040 N 0714332.1335$ 423040NO714332.2 335 423107 NO714508.1 $423245 N 0714719.1$ $423012 N 0714448.1410$

8 $423155 N 0714709.1550$ $\begin{array}{rll}9 & 423243 N 0714713.1 & 500 \\ 10 & 423144 N 0714404.1 & 350\end{array}$ $\begin{array}{lll}10 & 423144 N 0714404.1 & 350 \\ 11 & 423154 N 0714405.1 & 363\end{array}$ 12 423111N0714538.1 410

$13423104 N 0714404.1 \quad 345$ $\begin{array}{lll}14 & 423037 N 0714426.1 & 325 \\ 15 & 422913 N 0714526.1 & 525\end{array}$ $\begin{array}{lll}16 & 422902 N 0714547.1 & 520 \\ 18 & 423230 N 0714423.1 & 385\end{array}$

19 423254NO714452.1 385 $20 \quad 423229 N 0714431.1 \quad 365$ $21423119 N 0714601.1 \quad 410$ 23 423107NO714549.1 398

$24 \quad 423116$ NO714610.1 430 $\begin{array}{lll}25 & 423115 N 0714611.1 & 450 \\ 26 & 422912 N 0714539.1 & 534\end{array}$ $\begin{array}{lll}26 & 422912 N 0714539.1 & 534 \\ 27 & 422912 N 0714534.1 & 538\end{array}$ $28 \quad 422911$ NO714525.1 538

$29 \quad 422913$ N0714523.1 530 $\begin{array}{lll}30 & 422900 N 0714458.1 & 548 \\ 31 & 422949 N 0714517.1 & 493\end{array}$ $\begin{array}{lll}31 & 422949 N 0714517.1 & 493 \\ 32 & 422958 N 0114646.1 & 635\end{array}$ 33 423012N0714645.1 640

34 423049N0714645.1 640 35 423051N0714642.1 36
37 $423053 N_{0} 714640.1$ 38 423108NO714701.1

$39 \quad 423105 N 0714621.1 \quad 515$ $40 \quad 423054 N 0714622.1$ $41423051 N 0714613.1$ $42423102 N 0714612.1$

$44423103 N 0714546.1$ $45 \quad 423105 N 0714532$. $46423100 N 0714530$. $47423045 N 0714522.1$ 48 423036NO714511.1

$49423051 N 0714512.1$ $50423101 N 0714505.1$ $51423116 N 0714507.1 \quad 382$ $\begin{array}{lll}52 & 423130 N 0714528.1 & 412 \\ 53 & 423124 N 0714548.1 & 398\end{array}$

54 423138N0714520.1 352 55 423137N0714522.1 352 $\begin{array}{lll}56 & 423137 N 0714521.1 & 352 \\ 57 & 423151 N 0714533.1 & 435\end{array}$ 58 423154NO714540.1 500

59423208 N0714513.1 385 $60 \quad 423139$ N0714624.1 $61423145 N 0714700.1$ 62423201 NO714627.

$64423255 N_{0714618.1}$ 64 423255N0714618.1 $66423324 N 0714634.1$ $67423350 N 0714516.1 \quad 475$ 68 423357NO714507.1 491

$69423400 N 0714453.1508$ $70423316 N 0714436.1 \quad 476$ $71 \quad 423234 N 0714456.1338$ $\begin{array}{lll}72 & 423159 N 0714335.1 & 486 \\ 73 & 423146 N 0714328.1 & 513\end{array}$
MDPW
MDPW
MDPW

GERRY WILLIAM

$\begin{array}{cc}1936 & V \\ 1948 & V \\ 1948 & V \\ -- & D \\ -- & D\end{array}$

LAMOUREUX W

LAMOUREUX

US PLASTIC CO

POWERS R

MCCAFFERY $W$

CHANEY

MERRIAM HFC 1841

PIERCE GE

TISOALE L

VERGE

VISCOLOIO CO

HAOLEY GEORGE

I

1
1
330
30
30
36
48
60
6
36
48
20
24
30

$\begin{array}{rr}38 & 1 \\ 52 & 1 \\ 59 & 1 \\ 4 & 2 \\ 3 & 2\end{array}$

$\begin{array}{llr}-- & - & -- \\ -- & -- & 0 \\ -- & -- & -- \\ -- & -- & 3\end{array}$

$\begin{array}{rlll}8 & U & -- & - \\ 10 & U & -- & - \\ 0 & 2 & -- & - \\ 4 & U & -- & U\end{array}$

$128 \mathrm{U} \quad 9 \quad--\quad 40$

$\begin{array}{rll}16 & U & = \\ 26 & U & = \\ 4 & U & = \\ 11 & Z & = \\ 10 & U & =\end{array}$

WYMAN J $P$

PHELPS E H

GT AMER NOVEL

HARRIS CW W

LEOMINSTER ICE $1959 R$

LEOMINSTER ICE $1963 \mathrm{R}$

LECLERC ARMAND $1965 \mathrm{P}$

$\begin{array}{ll}\text { BILOTTA RAYMOND } & 1957 \\ \text { WILSON TREVOR C } & 1956\end{array}$

CONNOLLY JAMES 1966

OONATELLY V N 1960 P

LAGOY CONST CO 1957

$\begin{array}{ll}\text { POWERS GERDON } & 1966 \\ \text { PRINGLE J W } & 1962\end{array}$

$\begin{array}{lll}\text { WILLRUTH THEO } & 1957 & P \\ \text { TERRY THOMAS J } & 1966 & P\end{array}$

$\begin{array}{lll}\text { FRYE ROY L } & 1952 & c \\ \text { RIVAKD ALBERT J } 1967 & P\end{array}$

PORTER HOWARD 1958

LAVALLEE JOS A $1964 \%$

NEARY ARTHUR $1964 \mathrm{~V}$

MARSHALL OLEN P 1952

COURTOIS 1965.

A J RENZI CO 1965

$\begin{array}{lll}\text { LANCO PLASTICS } & 1966 & P \\ \text { FIXIT AUTO BODY } & 1966 & P\end{array}$

FIXIT AUTO BODY 1966

$\begin{array}{ll}\text { TOCCI AMERIGO } & 1964 \\ \text { MILLER PLASTICS } & 1957\end{array}$

AMOCO CHEM CORP $1970 P$

REFRIGERI UGO 1965

$\begin{array}{lll}\text { GABARELLI } & 1965 & - \\ \text { VENTRA PLASTIC } & 1967 & \text { P } \\ \text { STD PYROXOLOID } & 1964 & \end{array}$

STAR MFG CO

IILTON T COOK

IILTON COOK

KAVANAUGH D L 1965

$1965=$

LYNCH BRUCE H 1967

KINGMAN E B CO 1964

LEOM SKI AREA 1965

$\begin{array}{lll}\text { DESPO ALEXANDER } & 1956 \\ \text { RUBIN L } & 1965-\end{array}$

DOYLE LOUISE $1965 P$

DOYLE LOUISE I 1965

GRN ACRE NURS 1960

$\begin{array}{ll}\text { FITZGERALD E V } & 1966 \\ \text { TWINING MARION } & 1964\end{array}$

DEBONIS FLAVIO 1965 -

MAINVILLE A L

PLASTICRAFT

DESHARNAI5 GEO 1962

ROMANO NICHOLAS 1965
26

$\begin{array}{rr}26 & 0 \\ 8 & u \\ 13 & 0\end{array}$

13

$$
\begin{array}{rr}
24 & - \\
8 & x \\
-- & W \\
24 & W
\end{array}
$$

24

$\begin{array}{rr}8 & 4 \\ 120 & 4 \\ 120 & 0\end{array}$

120
12
41

202

270
415
230

328
235

103

153
300

385

$\begin{array}{rrrrr}385 & w & 190 & -- & 85 \\ 67 & w & 18 & =- & 30 \\ 162 & w & 1- & =- & 14 \\ 250 & w & 10 & -- & 14\end{array}$

240
17

135
145

950

265

24
14

$18 \mathrm{~T}=$

1000
450

450
325

325
1120

$120 w=-$

645
503

965

$300 W$
$\angle 07-6$

125

$\begin{array}{ll}17 & 1 \\ 32 & 1\end{array}$

140

145

750

68

390
230

$85 w$

145
327
27

390
328

$360-22$
IMEAS-
IUT) IURED

(GPM) I (FT) ' (HR) 
LOCAL NELL

LATITUDELATITUDE-
LONGITUDE
ALTI (FT) TUDE OWNER OR USER

YEAR

METHOD DIA-DWELL METHOD DIAM-IFIN-IDEPTHIUSE TO BEARING \begin{tabular}{l|l|l} 
ETER IISH & BED- MATERIAL \\
(IN) & IFT) & ROCK
\end{tabular}

LEVEL IDATE IUSE YIELPUMPAGE IMEAS-I UMINSTER --CONTINUED

$423134 N 0714356.1335$ 75 423110N0714345.1 300 $77423057 N 0714433.1361$ $78423046 N 0714440.1380$ $\begin{array}{lll}79 & 423016 N 0714402.1 & 324 \\ 80 & 423014 N 0714456.1 & 400\end{array}$ $81423221 N 0714521.1 \quad 319$ $\begin{array}{lll}82 & 423254 N 0714713.1 & 478 \\ 83 & 422927 N 0714337.1 & 360\end{array}$

$84 \quad 422929$ N0714332.1 348 85 422835N0714345.1 325 $\begin{array}{lll}86 & 422840 N 0714346.1 & 330 \\ 87 & 422836 N 0714349.1 & 328\end{array}$ 88 423032N0714507.1 357

$89423035 N 0714509.1358$ $90423039 N 0714501.1 \quad 352$ $\begin{array}{lll}91 & 423043 N 0714502.1 & 348 \\ 92 & 423039 N 0714455.1 & 346\end{array}$ $93423034 N 0714458.1351$

$94 \quad 423144 N 0714237.1 \quad 395$ 96 423028N0714517.1 382 97 42302INO714403.1 290 98 423022N0714359.1

99423019 N0714407.1 301 $\begin{array}{lll}100 & 423018 N 0714419.1 & 325 \\ 101 & 423031 N 0714402.1 & 290\end{array}$ * 102 423002NO714337.1 295 103423247 N0714316.1 390

$\begin{array}{lll}104 & 423144 N 0714445.1 & 298 \\ 105 & 423013 N 0714501.1 & 395\end{array}$ $\begin{array}{lll}105 & 423013 N 0714501.1 & 395 \\ 106 & 422936 N 0714433.1 & 421\end{array}$ $106422936 N 0714433.1$
$107423035 N 0714416.13$
108 * 108 422954N0714344.1 300

W 109422954 N0714339.1 290 11 422955N0714334.1 280 w 112 423111N0714408.1 292

* $114 \quad 422940$ N0714326.1 310 * 115423217 NO714256.1 390 - 116 423149N0714518.1 328

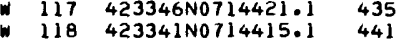

w 119423051 N0714558.1 426 $\begin{array}{lll}W 120 & 423143 N 0714634.1 & 425 \\ w 121 & 423019 N 0714429.1 & 345\end{array}$

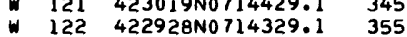
w 123 422949NO714858.1 754

$\begin{array}{rrr}124 & 423240 \text { N0714309.1 } & 410 \\ 125 & 423242 \text { N0714305.1 } & 410\end{array}$

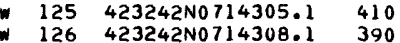
$\begin{array}{r}127 \\ -128323239 N 0714455.1 \\ \hline\end{array}$

w $129 \quad 423156 N 0714441.1308$ * $130 \quad 423147 N 0714429.1297$ 132 423131N0714416.1 290
-132.1 * 133 423150N0714444.1 302

W 134 423146N0714439.1 300 $W \quad 135 \quad 423143 N 0714428.1295$ $\begin{array}{lll}136 & 423223 N 0714437.1 & 31 B\end{array}$ $\begin{array}{rrr}137 & 423217 N 0714446.1 & 315 \\ 138 & 423256 \text { No714548.1 } & 385\end{array}$

- 139423227 N0714451.1 318 149 423132N0714412.1 294

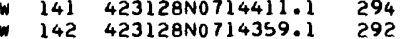
w $143423331 N 0714354.1386$

* $144 \quad 423331 N 0714350.1380$ $\begin{array}{lll}145 & 423331 N 0714347.1 & 375 \\ 146 & 423029 N 0714451.1 & 370\end{array}$ $\begin{array}{lll}146 & 423029 N 0714451.1 & 370 \\ 147 & 423028 N 0714451.1 & 372\end{array}$ w 148 423026NO714451.1 373
GOODWIN JEAN $1964-6 \quad 6 \quad \times \quad 122$

PAM PLASTIC CO $1964 \quad \bar{P}$

LAVERDIERE A 1965 P

CLEAR SHIELD CO 1966 P

HILLTOP PLASTIC 1966 C

$\begin{array}{lll}\text { LEOM MOTEL RLTY } & 1965 & \text { W } \\ \text { LESTER GRANT } & 1958\end{array}$

FOSTER GRANT 1958

$\begin{array}{lll}\text { LEOMINSTER CITY } & 1961 \\ \text { LEOMINSTER CITY } & 1957\end{array}$

LEOMINSTER CITY 1957 -

LEOMINSTER CITY $1957-$

LEOMINSTER CITY 1957 -

$\begin{array}{lll}\text { LEOMINSTER CITY } & 1957 \\ \text { LEOMINSTER CITY } & 1956\end{array}$

LEOMINSTER CITY 1956

LEOMINSTER CITY 1956

LEOMINSTER CITY 1956

LEOMINSTER CITY 1956

LEOMINSTER CITY 1956

LEOMINSTER CITY 1954

LEOMINSTER CITY 1954

LEOMINSTER CITY 1954

LEOMINSTER CITY 1954

LEOMINSTER CITY 1954

EEOMINSTER CITY 1954

LEOMINSTER CITY 1954

LEOMINSTER CITY 1954

LEOMINSTER CITY 1954

LEOMINSTER CITY 1954 -

LEOMINSTER CITY 1954

$\begin{array}{lll}\text { LEOMINSTER CITY } & 1954 \\ \text { LEOMINSTER CITY } & 1954\end{array}$

LEOMINSTER CITY 1954

LEOMINSTER CITY 1954

LEOMINSTER CITY 1954

LEOMINSTER CITY 1954

LEOMINSTER CITY 1954

LEOMINSTER CITY 1955

LEOMINSTER CITY 1955

LEOMINSTER CITY 1955

LEOMINSTER CITY 1955

LEOMINSTER CITY 1955

LEOMINSTER CITY 1953

LEOMINSTER CITY 1959

$\begin{array}{lll}\text { LEOMINSTER CITY } & 1959 \\ \text { LEOMINSTER CITY } & 1963\end{array}$

CORMIER EDWARO $1970 \mathrm{R}$

GENDRON RENE A 1963 R

KIMBALL CHARLES

LEOMINSTER CITY 1950 W

LEOMINSTER CITY 1950

LEOMINSTER CITY 1950

LEOMINSTER CITY 1950

LEOMINSTER CITY 1950

LEOMINSTER CITY 1950

LEOMINSTER CITY 1950

LEOMINSTER CITY 1950

LEOMINSTER CITY 1950

LEOMINSTER CITY 1950

LEOMINSTER CITY 1950

LEOMINSTER CITY 1950

$\begin{array}{lll}\text { LEOMINSTER CITY } & 1950 \\ \text { LEOMINSTER CITY } & 1950\end{array}$

LEOMINSTER CITY 1950

LEOMINSTER CITY 1950

LEOMINSTER CITY 1950

LEOMINSTER CITY 1950

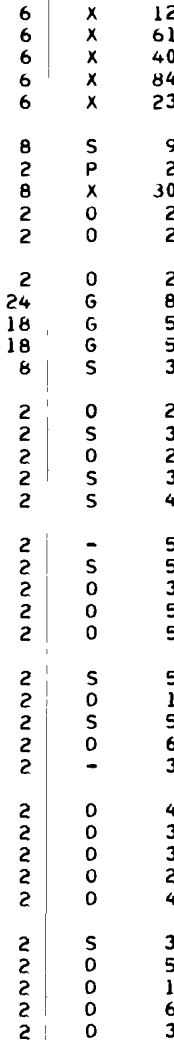

28

\begin{tabular}{l|l}
33 \\
29 \\
36
\end{tabular}

59

53
34
53

53

58

57
69

$37 \mathrm{~T}$

31

31

10

$38 \mathrm{~T}$

24

29

21

150

160

35
52
50

30

40

30

65

62

if
LEOMINSTER CITY 1954 -

$\begin{array}{rrr}122 & - & 10 \\ 610 & W & - \\ 400 & w & 115 \\ 840 & w & 100 \\ 235 & W & 62 \\ 97 & W & -- \\ 22 & T & 22 \\ 300 & w & 16 \\ 23 & T & -- \\ 25 & T & -\end{array}$

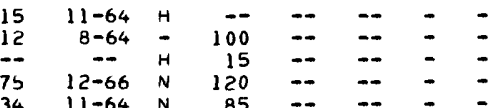

\begin{tabular}{l|ll}
27 & $T$ & $=-$ \\
81 & $W$ & $=-$ \\
54 & $W$ & -- \\
53 & $W$ & 53 \\
38 & $T$ & 33
\end{tabular}

$34 \mathrm{~T}=-$

$\begin{array}{lllll}23 & T & -- & = & =-\end{array}$

$\begin{array}{cc}-- & N \\ 1-65 & U \\ --5 & \end{array}$

40
5
90

$2 S$
$2 R$

-57
$\mathrm{~N}$

$11-57$

$11-57$
$11-57$
$2-57$

$12-56$

$\begin{array}{llll}33 & 1 & - & 4 S \\ 51 & 1 & = & 25 \\ 15 & 1 & =- & -- \\ 68 & 1 & -- & --\end{array}$

$\begin{array}{llll}47 & T & 47 & 25\end{array}$

$28 T \quad 14$

$\begin{array}{llll}30 & 1 & = & =\end{array}$

$\begin{array}{llll}2 & -2 & -- & -\end{array}$

$\begin{array}{rrrrr}17 & T & - & -- & = \\ 19 & T & - & - & = \\ 35 & T & - & S & = \\ 15 & T & - & R & = \\ 18 & T & -- & K & -\end{array}$

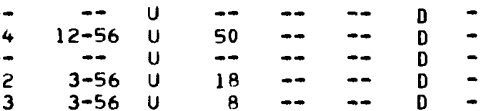

$\begin{array}{lllllll}1 & 1-54 & u & 15 & \because- & -- & 0 \\ - & - & u & -- & -- & - & 0\end{array}$

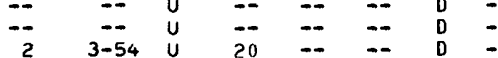

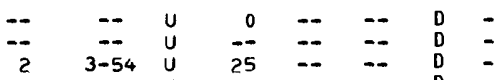

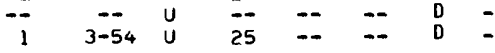

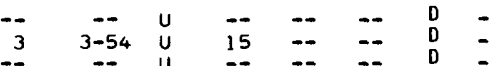

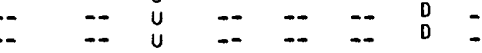

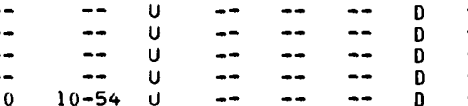

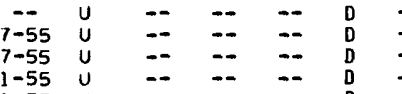

$\begin{array}{llllll}1-55 & U & = & -- & = & 0 \\ 1-55 & u & -- & -- & -- & 0\end{array}$

$\begin{array}{cccccccc}1 & 2-55 & u & -- & -- & -- & 0 & = \\ - & -- & u & - & - & - & 0 & -\end{array}$

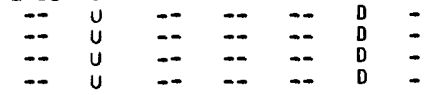

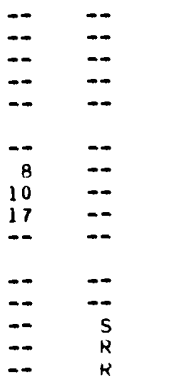

$\because$

$\begin{array}{rr}-70 & H \\ -69 & H\end{array}$

$\begin{array}{lllll}-70 & H & -- & =- & = \\ -69 & H & 75 & \bar{Z} & = \\ -- & - & -- & -- & -\end{array}$

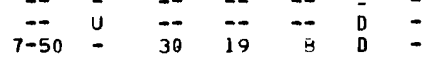

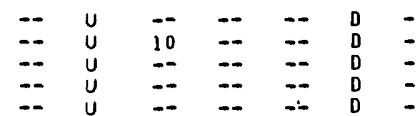

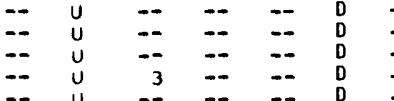

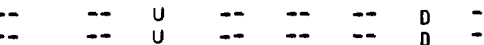

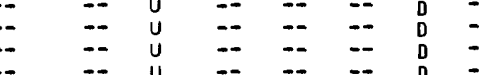

$=15$

41 ${ }^{36}+\frac{T}{4}$

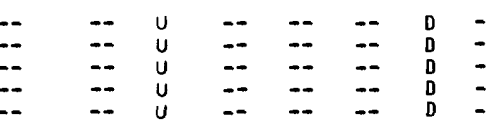




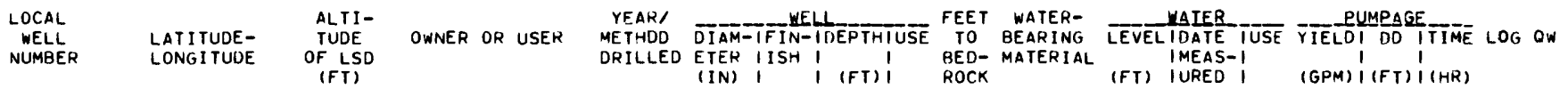

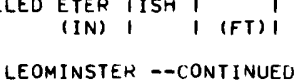

* $149423204 N 0714633.1$ $150423202 N 0714634.1$ 151423159 N0714634.1 $152423241 N 0714655.1$

w 154 423231N0714456.1 - $155423225 \mathrm{~N} 0714522.1$ - 156 423228N0714522. $157423113 N 0714601.1$
$158423245 N 0714454.1$

159423109 N071455B.1 - $160423115 N 0714552.1$ $161423112 N 0714550.1$
$163423248 N 0714521.1$ 164 422933N0714407.1

* $165423310 \mathrm{N0714322.1}$ $166422955 N 0714325.1$ 423246 N0714456. $423241 N 0714451$.

4 423356N0714436.1 5 423342N0714411.1 $6423331 N 0714356.1$ 7
8 $423319 N 0714416.1$

9 423133NOT14355.1 $10423019 N 0714404.1$ I1 423033N0714449.1 2 423029N0714510. $\times \quad 14 \quad 422947 N 0714532.1$ $x \quad 15423323 N 0714503.1$
$\times \quad 16 \quad 423125 N 0714406.1$

$1423414 \mathrm{NO} 714238.1$ $2423230 N 0714042.1$ $3423229 \mathrm{NO} 714042.1$ $423209 N_{0} / 14136.1$

6 423257N0714113.1 305 $\begin{array}{lll}6 & 423257 N 0714113.1 & 305 \\ 7 & 423252 N 0714111.1 & 305\end{array}$ $\begin{array}{lll}7 & 423252 N 0714111.1 & 305 \\ 8 & 423311 N 0714139.1 & 335\end{array}$ 9 423333N0714138.1 298

$11423432 \mathrm{NO} 714140.1372$ $\begin{array}{lll}12 & 423451 N 0714150.1 & 391 \\ 13 & 423436 N 0714154.1 & 395\end{array}$ 13423436 NOT14154.1 395 $\begin{array}{lll}15 & 423508 N 0714047.1 & 507\end{array}$

$16423532 N 0714041.1$ $17423533 N 0714055.1$ $18423422 N 0714246.1$ 20 423404NO/14403.1

$21423402 N 0714411.1$ 22423418 N0714529. $23423408 N 0714555$. 25 423507N0714455.1

$26423520 N 0714506.1$ 27 423526N0714501.1 28 423618N0714611. 29
30 $423625 N 0714541.1$

$31423627 N 0714511.1$ $32423625 N 0714506$. $\begin{array}{ll}33 & 423628 N 0714453.1 \\ 34 & 423632 N 0714455.1\end{array}$ 34 423632N0714455.

36 423626N0714421.1 37 423628N0714418. $38423621 N 0714318$. $39423703 N 0714462.1$

\section{432 472 472 \\ 321 399
320} 400
399 399 386
385

359

325

320

440

$\begin{array}{llll}485 & \text { RAND-WHITNEY } & 1964 & \mathrm{v} \\ & \text { LEOMINSTER CITY } & 1963\end{array}$

LEOMINSTER CITY 1950

LEOMINSTER CITY 1950

LEOMINSTER CITY 1950

LEOMINSTER CITY 1950

LEOMINSTER CITY 1950

LEOMINSTER CITY 1950

LEOMINSTER CITY 1950

$\begin{array}{lll}\text { LEOMINSTER CITY } & 1950 \\ \text { LEOMINSTER CITY } & 1950\end{array}$

LEOMINSTER CITY 1950 W

LEOMINSTER CITY 1950

LEOMINSTER CITY 1950

$\begin{array}{llll}\text { LEOMINSTER CITY } & 1950 \\ \text { LEOMINSTER CITY } & 1950 \quad \mathrm{w}\end{array}$

LEOMINSTER CITY 1950 W

LEOMINSTER CITY 1963

FITCHBURG CITY 1968

$\begin{array}{ll}\text { FITCHBURG CITY } & 1968 \\ \text { FITCHBURG CITY } & 1968\end{array}$

LEOMINSTER CITY $1963 \mathrm{~V}$

LEOMINSTER CITY $1963 \mathrm{~V}$

LEOMINSTER CITY 1963

LEOMINSTER CITY 1963

LEOMINSTER CITY 1963 W

LEOMINSTER CITY 1963 W

LEOMINSTER CITY 1963

LEOMINSTER CITY 1963

LEOMINSTER CITY 1963
LEOMINSTER CITY 1963 w

2
2
2
2
2
2
2
2
2
2
2
2
2
2
2
2
2
2
2
2
2
2
2
2
2
2
2
2
2
2
2
2
2
2
2
2
2
2
2
2
2
2
2
2
2
2
2
2
2
2
2
2
2
2
2
2
2

317

315

(1)

480

480
360

449
528

630

423

500

574

641

465

578
568

550

550
515
555

545

592

592
570
515

570
600
LUNENBURG

LUNENBURG TOWN

KEATING $P$ S

P KEATING CO

NIEMI GENE

$1961 \mathrm{C} 24$

PERRAULT HENRY

1971
1964

CONNORS JOHN 1968

PERRAEULT RO甘T 1969 C

PATRY ROBT

1968

DELMONICO LEON

AMBERT ERNEST

$1953 \mathrm{C}$

FEEMAN ELMER L 1958 P

WHITNEY RAYMOND 1962 C

PEARSON WILLIAM 1964

PROCTOR CARL W 1951

$\begin{array}{ll}\text { CADWELL HOWARD } & 1956 \\ \text { RIVERS ROBT } & 1968 \\ \text { DEBONIS A } & 1960\end{array}$

1968

MURPHY GEO A

PIERMARINI DINO 1950 C

MCGINNIS ROBT E 1965

HANEY OSWALO 1958

WHITE EDGAR 1955 P

PENDLETON F E 1957 P

$\begin{array}{lll}\text { PICARD ARTHUR R } & 1964 & \text { P } \\ \text { MAPLEWOOD GOLF } & 1965 & \end{array}$

$\begin{array}{ll}\text { MAPLEWOOD GOLF } & 1965 \\ \text { CHARRON } & 1965\end{array}$

GIONET RUDOLPH 1967 -

$\begin{array}{lll}\text { KOIVISTO E } & 1963 & P \\ \text { KELLEY HENRY D } & 1969 & P\end{array}$

$\begin{array}{lll}\text { KELLEY HENRY U } 1969 & P \\ \text { MAROUART ROBT T } 1963 & P\end{array}$

LEHLANC ROLAND $1969 \mathrm{P}$

SCHUSTER W A SR 1955 P

$\begin{array}{lll}\text { TACKETT RL L } & 1965 & \mathrm{P} \\ \text { HOPE RUBBER CO } & 1965 & \mathrm{P}\end{array}$

DAOUST HAROLD 1965 P

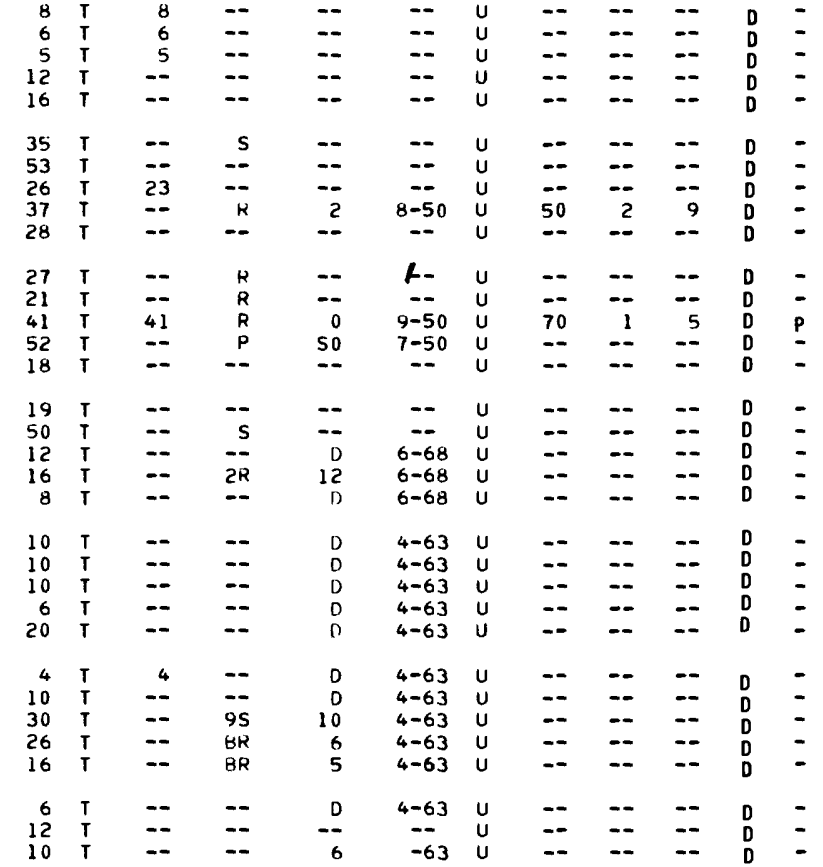

$\begin{array}{rrrrrrrr}2 & 7-61 & \mathrm{P} & 290 & 14 & 191 & \mathrm{D} & \mathrm{P} \\ 11 & 1-71 & \mathrm{~N} & 1560 & 21 & 12 & \mathrm{D} & \mathrm{P} \\ 4 & 9-64 & \mathrm{~N} & 940 & 14 & 24 & \mathrm{D} & - \\ 2 & 9-71 & \mathrm{~N} & 62 & 4 & 3 & \mathrm{D} & \mathrm{P} \\ 140 & 2-68 & - & 4 & -- & -- & - & -\end{array}$

$\begin{array}{rrrrrr}30 & 11-67 & H & .8 & -- & -- \\ 2 & 11-68 & H & 50 & 18 & = \\ 33 & 6-64 & H & 4 & -- & -- \\ 4 & 8-69 & H & 6 & 18 & -- \\ 35 & 1-68 & H & 15 & -- & --\end{array}$

13

$11-53$

$11-53$
$11-50$

$11-58$
$3-62$

366
35
90
250
390

$\begin{array}{rr}4 & - \\ 41 & - \\ 159 & - \\ 119 & -\end{array}$

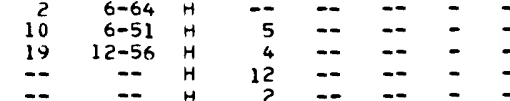

$300 \div 218$

$95 w 30$

150 -

$145-13=$

$\begin{array}{llll}160 & w & -- & -- \\ 200 & w & 22 & -- \\ 140 & w & 22 & -- \\ 500 & - & -- & -\end{array}$

$\begin{array}{rrrrrrr}147 & 1-68 & H & 100 & -- & -- & - \\ 15 & 5-50 & H & 4 & -- & -- & -\end{array}$

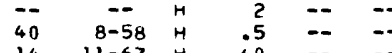

$527 w$

185

265

245

$--$

5
63
82

--

$\begin{array}{rrrrr}18 & 3-55 & H & 2 & =- \\ 35 & 1-57 & H & 3 & =- \\ 16 & 11-64 & H & 3 & =- \\ -- & -- & 4 & 1 & =-\end{array}$

$20 \quad 10-65$

80
83
165
335

8
7
19
102
124

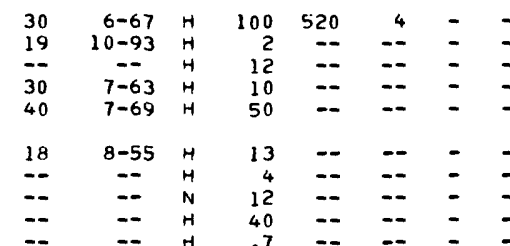




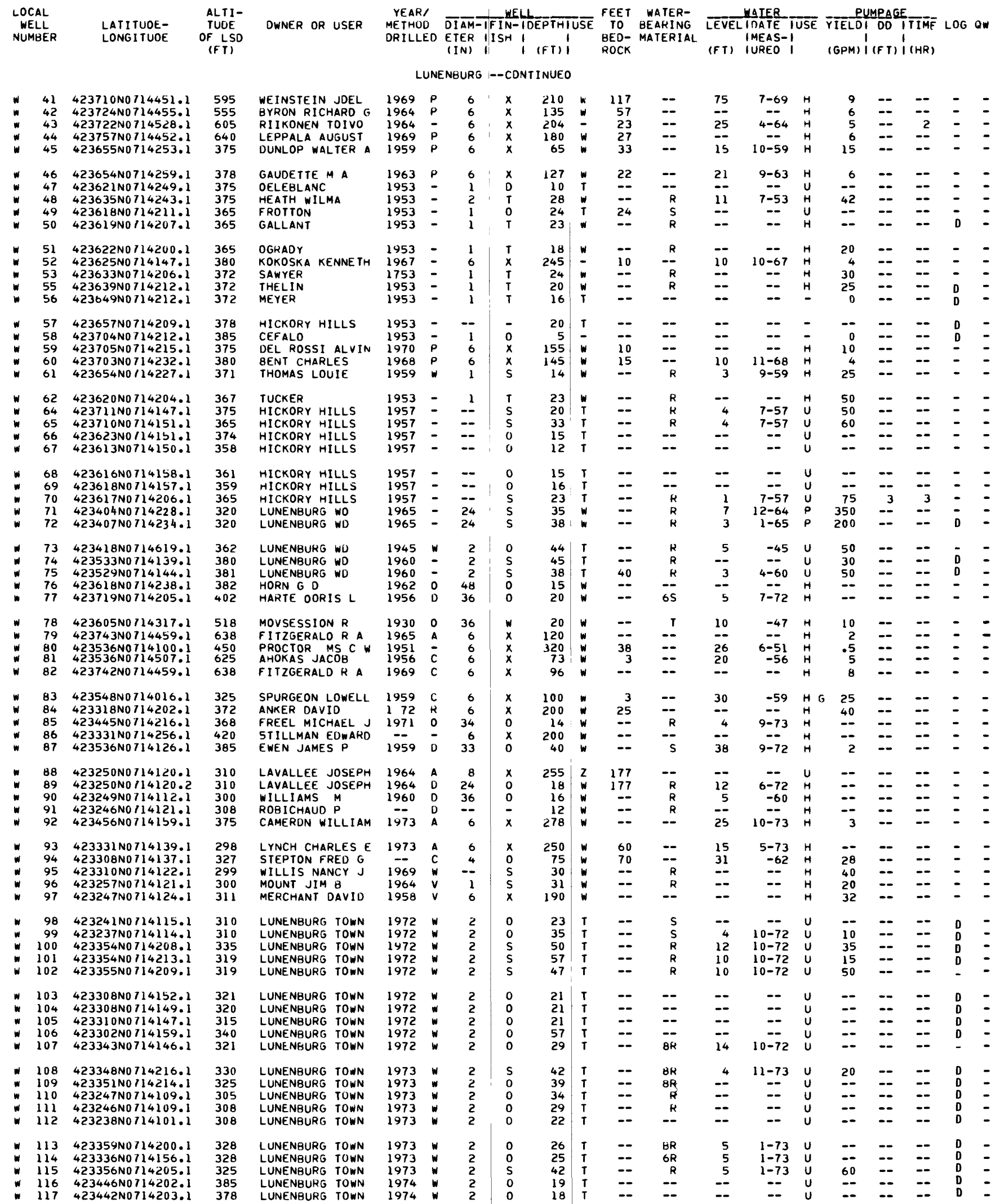


LOCAL NUMBL
LATI TUDELONGITUOE
ALTI-
TUDE OF LSD
OWNER OR USER

YEARI ORILLED ETER IISH I IFI)
(IN) I

LUNENBURG --CONTINUEO

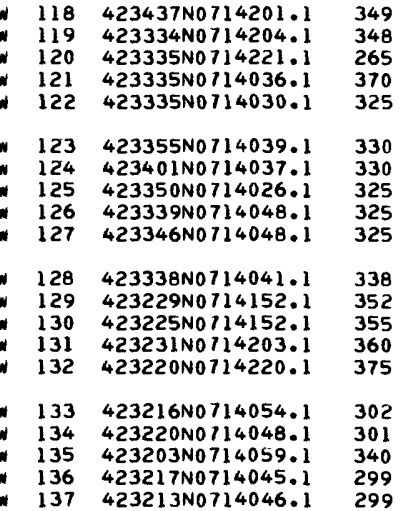

LUNENBURG TOWN LUNENBURG TOWN LUNENBURG TOWN LUNENBURG TOWN LUNENBURG TOWN

LUNENBURG TOWN LUNENBURG TOWN LUNENBURG TOWN LUNENBURG TOWN
LUNENBURG TOWN

LUNENBURG TOWN LUNENBURG TOWN LUNENBURG TOWN LUNENBURG TOWN

LUNENBURG TOWN LUNENBURG TOWN LUNENBURG TOWN LUNENBURG TOWN LUNENBURG TOWN

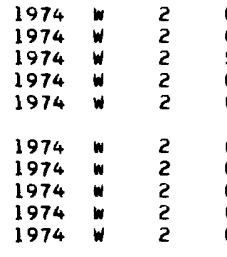

$\begin{array}{l:l}1974 & 2 \\ 1974 & \\ 1974 & 2 \\ 1974 & 2 \\ 1974 & 2\end{array}$

1975 w
1975
1975 w
1975 w

\begin{tabular}{|c|c|c|c|}
\hline 14 & $T$ & -- & - \\
\hline $\begin{array}{l}21 \\
49\end{array}$ & $T$ & $=$ & $\overline{8}$ \\
\hline 33 & $T$ & -- & - \\
\hline 48 & $T$ & -- & - \\
\hline 43 & $\boldsymbol{T}$ & -- & \\
\hline 35 & $T$ & -- & \\
\hline 3 & $T$ & $=$ & \\
\hline 31 & $T$ & 31 & \\
\hline 77 & T & -- & 8 \\
\hline & & & \\
\hline 13 & I & -- & 8 \\
\hline & $T$ & - & 6 \\
\hline
\end{tabular}

$\begin{array}{rrrr}81 & 1 & -- & 6 x \\ 87 & 1 & - & 95 \\ 71 & 1 & 71 & 95 \\ 91 & 1 & -- & 5\end{array}$

\begin{tabular}{|c|c|c|c|c|c|}
\hline$=$ & u & -- & -- & -- & D \\
\hline $1=74$ & u & 0 & $\overline{-}$ & $=$ & D \\
\hline-- & 政 & -- & -- & -- & D \\
\hline & & & & & \\
\hline$=$ & u & -- & $=$ & $=$ & 0 \\
\hline $5-74$ & u & 10 & $=$ & $=$ & $D_{0}^{0}$ \\
\hline-0 & $u$ & -- & -- & + & D \\
\hline & u & -- & -- & -- & \\
\hline- & u & -- & - & -- & 0 \\
\hline $7-74$ & u & $=$ & - & -- & D \\
\hline $9-$ & $u$ & 25 & - & -- & D \\
\hline $\begin{array}{l}8-74 \\
8-74\end{array}$ & u & .5 & $\because$ & $=$ & $\begin{array}{l}\text { D } \\
\text { D }\end{array}$ \\
\hline $1-75$ & u & -- & -- & -. & 0 \\
\hline & $u$ & & & & D \\
\hline & $u$ & 25 & & 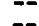 & 0 \\
\hline $1-75$ & u & 6 & 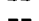 & 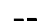 & D \\
\hline
\end{tabular}

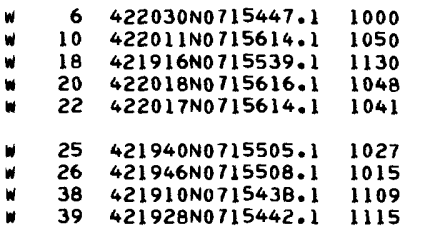

MORRIS $O H$ ERSKINE L M JR SAVIGNAC

JONES ROBERT E GLEASON HAROLO

ANNA MARIA COLL

ANNA MARIA COLL PAXTON TOWN
PAXTON TOWN

\begin{tabular}{ccc} 
& & \multicolumn{2}{c}{ PAXTON } \\
1942 & $C$ & 6 \\
1971 & $p$ & 6 \\
-- & 0 & - \\
1964 & - & 6 \\
1954 & $C$ & 6 \\
1954 & $C$ & 6 \\
1959 & $C$ & 8 \\
1950 & $w$ & 2 \\
1950 & $W$ & 2
\end{tabular}

PEPPERELL

HEBERT LOUIS
WINN HC
MAYNARD
HEBERT L
PEPPERELL YARN
NASHUA R PAPER
BEAMUS
HERRIG
LOUIS
RICHARDSON
TUNE
WILLIAMS
MCGRAW
MORANT

POER RICHARD POER RICHARO
ROCHETTE R

FOX W

STEINHOLTZ LAAS STE INHOLTZ LAAS BUCK R W

PEPPERELL TOWN PEPPERELL TOWN PEPPERELL TOWN PEPPERELL TOWN PEPPERELL TOWN

PEPPERELL TOWN PEPPERELL TOWN PEPPERELL TOWN PEPPERELL TOWN
TOLMAN ROBERT E

\section{TOLMAN ROBERT E}

EVELETH LARTER HILGENDORF R

$\begin{array}{lll}1908 & 0 & 36 \\ 1910 & 0 & 24\end{array}$ $\begin{array}{lll}1908 & 0 & 36 \\ 1-0 & 0 & 36\end{array}$ $1900 \quad 36$ $\begin{array}{lll}= & D & 60 \\ = & D & -\end{array}$

$=00$$$
\text { - }
$$$$
\begin{array}{ccc}
-- & 0 & 48 \\
1923 & 0 & 60 \\
-- & 0 & --
\end{array}
$$$$
1970 \mathrm{C}
$$$$
\begin{aligned}
& 1966 \\
& 1966 \\
& 1966 \\
& 1966
\end{aligned}
$$$$
1966
$$

1966 1966 1967
1966 - D 50

1967 A

$1967=$
$1951=$
$1972=$ PEPPERELL TONN ADRIAN GRANT $K$$$
1966
$$
$\begin{array}{lll}39 & 423852 N 0713808.1 & 340 \\ 40 & 423945 N 0713859.1 & 455\end{array}$

41424017 N0713750.1 410 42 424037N0713814.1 412 $44424124 N 0713857.1 \quad 360$ $45424142 \mathrm{NO} 713846.1350$
REESE EUGENE H 19650

$\begin{array}{llll}\text { HAUE ISEN C C } & -\overline{0} & - \\ \text { MINER LAURENCE } & 1971 & v & -\end{array}$

MINER LAURENCE

LEVI HERBERT "

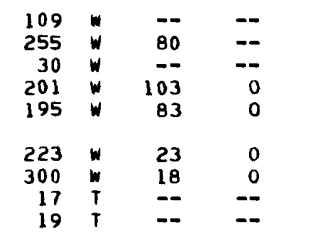

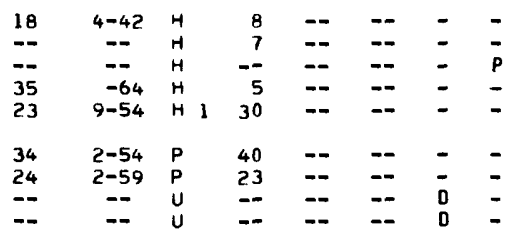


TABLE 1.--DESCRIPTION OF SELECTEO WELLS, TEST WELLS, AND BORINGS -- CONTINUED

LOCAL WELL
LATITUDELONGI TUDE
ALT1OF LSO (FT)
OWNER OR USER C

METHOO DIAM-IFIN-IDEPTHIUSE TO BEARING ORILLED ETER IISH I
(IN) I I IFT) I ROCK

$$
\begin{aligned}
& \text { IIN) IISH I (FT)! } \\
& \text { PEPPERELL }
\end{aligned}
$$

\begin{tabular}{|c|c|c|c|}
\hline & $\begin{array}{l}46 \\
47 \\
48 \\
49 \\
50\end{array}$ & $\begin{array}{l}424154 N 0713822.1 \\
4238450713821.1 \\
423851 N 0713837.1 \\
423829 N 0713529.1 \\
423832 N 0713540.1\end{array}$ & $\begin{array}{l}285 \\
348 \\
333 \\
209 \\
212\end{array}$ \\
\hline & $\begin{array}{r}51 \\
1 \\
2 \\
3 \\
4\end{array}$ & $\begin{array}{l}423757 N 0713630.1 \\
424106 N 0713726.1 \\
424105 N 0713656.1 \\
424059 N 0713636.1 \\
424045 N 0713606.1\end{array}$ & $\begin{array}{l}215 \\
370 \\
280 \\
245 \\
317\end{array}$ \\
\hline & $\begin{array}{l}5 \\
6 \\
7 \\
8 \\
9\end{array}$ & $\begin{array}{l}424013 N 0713649.1 \\
424004 N 0713625.1 \\
424021 N 0713641.1 \\
424033 N 0713632.1 \\
423951 N 0713622.1\end{array}$ & $\begin{array}{l}290 \\
295 \\
280 \\
260 \\
310\end{array}$ \\
\hline & $\begin{array}{l}10 \\
11 \\
12 \\
13 \\
14\end{array}$ & $\begin{array}{l}423948 N 0713550.1 \\
423958 N 0713522.1 \\
423953 N 0713441.1 \\
424050 N 0713519.1 \\
424016 N 0713441.1\end{array}$ & $\begin{array}{l}258 \\
250 \\
210 \\
232 \\
202\end{array}$ \\
\hline & $\begin{array}{l}15 \\
16 \\
17 \\
18 \\
19\end{array}$ & $\begin{array}{l}424031 N 0713418.1 \\
424015 N 0713422.1 \\
424020 N 0713416.1 \\
424013 N 0713410.1 \\
424004 N 0713355.1\end{array}$ & $\begin{array}{l}190 \\
182 \\
178 \\
179 \\
185\end{array}$ \\
\hline & $\begin{array}{l}20 \\
21 \\
22 \\
23 \\
24\end{array}$ & $\begin{array}{l}424001 N 0113332.1 \\
423945 N 0713710.1 \\
424028 N 0713352.1 \\
424032 N 0713358.1 \\
423936 N 0713629.1\end{array}$ & $\begin{array}{l}210 \\
315 \\
175 \\
190 \\
375\end{array}$ \\
\hline & $\begin{array}{l}25 \\
26 \\
27 \\
28 \\
29\end{array}$ & $\begin{array}{l}424004 N 0713554.1 \\
424026 N 0713455.1 \\
424105 N 0713549.1 \\
424043 N 0713446.1 \\
424007 N 0713417.1\end{array}$ & $\begin{array}{l}312 \\
200 \\
282 \\
218 \\
198\end{array}$ \\
\hline & $\begin{array}{l}30 \\
31 \\
32 \\
33\end{array}$ & $\begin{array}{l}423956 N 0713421.1 \\
423939 N 0713421.1 \\
424002 N 0713310.1 \\
423932 N 0713644.1\end{array}$ & $\begin{array}{l}218 \\
238 \\
202 \\
396\end{array}$ \\
\hline
\end{tabular}

423028 N0715225.1 912 $2422647 N 0713311.11060$ $3422749 N 0175138.1785$ $\begin{array}{lll}4 & 422906 N 0715432.1 & 1255 \\ 5 & 422903 N 0715428.1 & 1248\end{array}$ $6 \quad 422821 N 0715336.1 \quad 1300$ 7 422812NO715315.1 1330 8 422751N0715502.1 1150 9
11 422722 2N0712N0715236.1 1110

12422711 N0715233.1 1075 $13422659 N 0715240.1 \quad 1165$ $14 \quad 422652 N 0715245.1 \quad 1110$ 16 422653N0715250.1 1108

17 422649N0715257.1 1060 is 42270.3N0715319.1 1105 $\begin{array}{lll}18 & 422703 N 0715319.1 & 1105 \\ 19 & 422700 N 0715319.1 & 1102\end{array}$ 20 422654N0715342.1 1035 21422513 NOT15346.1 690

22422503 N0715333.1 800 23 422504N0715327.1 800 24 422457N0715317.1 790 $\begin{array}{lll}25 & 422458 N 0715315.1 & 800 \\ 26 & 422431 N 0715240.1 & 880\end{array}$

27422440 NO115242.1 921 $28422442 N 0715233.1920$ $29422445 N 0715235.1925$ $\begin{array}{lll}30 & 422507 N 0715240.1 & 980 \\ 31 & 422439 N 0715227.1 & 915\end{array}$

$32 \quad 422626 \mathrm{~N} 0715227.1 \quad 1040$ 33 422607N0715110.1 720 $34 \quad 422610 N 0714956.1 \quad 670$ 35 422625N0714955.1 652
KOHUT B N

BENCSIK FRANK PICKARD JOHN H WALENT WALTER BRIGHAM MASON

WILKINS GARY G PEPPERELL TOWN PEPPERELL TOWN PEPPERELL TOWN PEPPERELL TOWN

PEPPERELL TOWN PEPPERELL TUWN PEPPERELL TOWN PEPPERELL TOWN PEPPERELL TOWN PEPPERELL TOWN PEPPERELL TOWN PEPPERELL TOWN PEPPERELL TOWN

PEPPERELL TOWN PEPPERELL TOWN PEPPERELL TOWN PEPPERELL TOWN PEPPERELL TOWN

PEPPERELL TOWN PEPPERELL TOWN PEPPERELL TOWN PEPPERELL TOWN PEPPERELL TOWN PEPPERELL TOWN PEPPERELL TOWN PEPPERELL TOWN

PEPPERELL TOWN PEPPERELL TOWN PEPPERELL TOWN

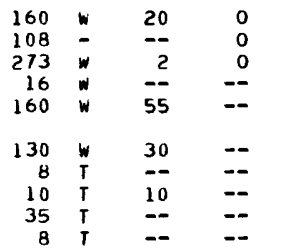

\begin{tabular}{c|ccc}
15 & $T$ & -- & $=-$ \\
15 & $T$ & -- & $=-$ \\
15 & $T$ & -- & $=-$ \\
15 & $T$ & -- & $=-$ \\
8 & $T$ & 8 & --
\end{tabular}

14

11

14
14
15

15
35
23
35

35
15

15

11
20
18

18
9

7
5
10

5
10
15

3

7
6
6

$1969 \mathrm{~V}$
$1969 \mathrm{~V}$
NIEMI ARVO

MINNS SUSAN

ANTONIO CHRIS

PORTER STANLEY 1971

ANDREWS

WILO LUCY

STIMPSON

1965
BELLTHROP ARTHUK
1957

GENDRON HOMER D 1968

PRINC CONGO CH 1950

SUNDIN

PRNCTN CTR SCH

$1965=$

WHITTEMORE O J $1955 \mathrm{P}$

BURR ALAN T 1959

MORSE RICHARD C $1955 \%$

$\begin{array}{lll}\text { SMITH JAMES B } & 1963 & P \\ \text { CONWAY RICHARO } & 1965-\end{array}$

KOBERTS RONALD

LAJOIE ALBERT * 1966

BOUCHER ALBERT 1955 P

$\begin{array}{lll}\text { GUSTAFSON ALLEN } & 1966 & - \\ \text { WIGGINS FRANK } & 1964 & -\end{array}$

COLE

COLE OURWARO

LUKEY JUL I AN

JOHNSON KEN

HUNT WILLIAM

$1963=$

$1964=$

$1964=$
$1971=$
1967

MACGEACHY $R$

PAINE DAVID I 1963

ALTBERG OLAF A $1 \overline{950} \bar{c}$
PRINCETON

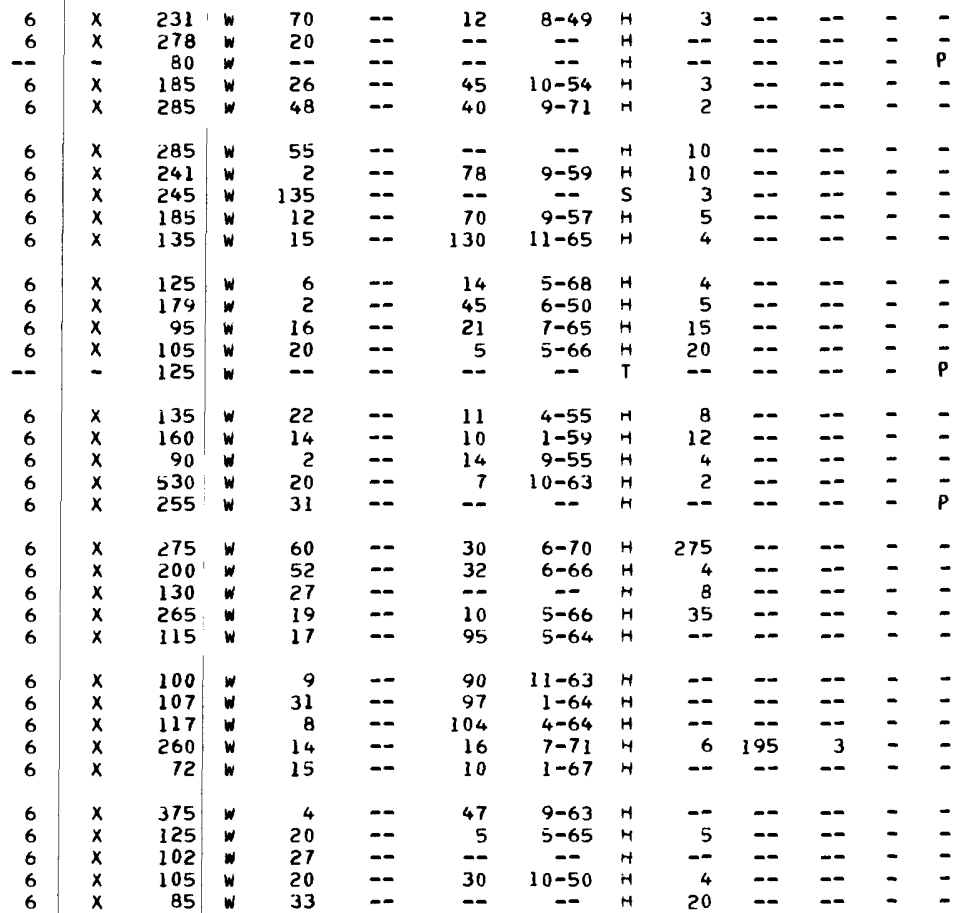

LEVELIDATE TUSE YIELDI DO ITIME LOG OW (FT) IURED I (GPM) I (FT) I (HR) 
$37422703 \mathrm{~N} 0715120.1820$ $\begin{array}{rlr}38 & 422709 N 0715149.1 & 995 \\ 39 & 422709 N 0715157.1 & 1025\end{array}$ $\begin{array}{rrr}39 & 422709 N 0715157.1 & 1025 \\ 40 & 422719 N 0715146.1 & 965\end{array}$ 41422713 N0715220.1 1020

$42 \quad 422739$ NO 715216.1953 $\begin{array}{lll}43 & 422752 N 0715225.1 & 970 \\ 44 & 422803 N 0715225.1 & 960\end{array}$ $\begin{array}{rrr}44 & 422803 N 0715225.1 & 960 \\ 45 & 422847 N 0715157.1 & 1072\end{array}$ $46 \quad 422924 N 0715213.1 \quad 1205$

$47 \quad 422932 N 0715208.1 \quad 1160$ $48 \quad 422943 N 0715203.11070$ $\begin{array}{rrr}49 & 422952 N 0715201.1 & 1050 \\ 50 & 422933 N 0715041.1 & 850\end{array}$ $51422931 N 0715046.1 \quad 840$

$52422904 N 0715117.1915$ 53 422849N0715105.2 815 $54 \quad 422832 N 0715006.1708$ 55 422829N0715006.1 711

57422804 N0715111.1 800 58 422815N0715057.1 760 $59422824 N 0715028.1700$ $\begin{array}{lll}60 & 422823 N 0715016.1 & 715 \\ 61 & 422824 N 0715019.1 & 713\end{array}$

$62 \quad 422822 N 0115019.1712$ 63 422825N0715012.1 715 $64 \quad 422756 N 0715458.11125$ 66 422656NO715541.1 1005

$67 \quad 422713$ N0715534.1 1048 68 422650N0715306.1 1078 $69422953 N 0715204.1 \quad 1042$ 71422703 N0715300.1 1133 $\begin{array}{rrrr}3 & 1 & 422351 N 0715501.1 & 955 \\ * & 4 & 422044 N 0715641.1 & 1038\end{array}$ $\begin{array}{llll}w & 14 & 422520 \text { NO715556.1 } & 1205 \\ & 18 & 422458 \text { NOT15545.1 } & 1175\end{array}$

$1423307 \mathrm{~N} 0713834.1240$ $\begin{array}{lll}2 & 423252 N 0713954.1 & 278 \\ 3 & 423206 N 0713823.1 & 270\end{array}$ $\begin{array}{lll}4 & 423143 N 0713831.1 & 260 \\ 6 & 423550 N 0713905.1 & 384\end{array}$

7423603 N0713748.1 262 $\begin{array}{lll}8 & 423557 N 0713925.1 & 342 \\ 9 & 423607 N 0713901.1 & 422\end{array}$ $10423436 N 0713825.1 \quad 295$ $11423402 N 0713735.1270$

\begin{tabular}{lll}
12 & $423707 N 0713938.1$ & 382 \\
\hline
\end{tabular} 13 423413N0713840.1 335 is $423455 \mathrm{~N} 0713939.1348$

$16423557 \mathrm{N0713851.1} 403$

$\begin{array}{lll}17 & 423423 N 0713755.1 & 282 \\ 18 & 423320 N 0713859.1 & 387\end{array}$ $19423356 N 0713939.1 \quad 330$ $20 \quad 423430$ N0713956.1 355

$22423456 \mathrm{~N} 0114004.1305$ 23 423510N0714003.1 320 24 423515NO71340S.1 278 $\begin{array}{lll}25 & 423521 N 0713916.1 & 285 \\ 26 & 423520 N 0713953.1 & 288\end{array}$

$27 \quad 423439 N 0714005.1 \quad 320$ 28 423550NO713949.1 312 $\begin{array}{lll}29 & 423547 \mathrm{NO} 713953.1 & 305 \\ 30 & 423545 \mathrm{~N} 0713950.1 & 305\end{array}$ $31423546 N 0713945.1 \quad 290$

$32 \quad 423515$ NO $713958.1 \quad 288$ 33 423526NOI13946.1 278 $34 \quad 423513 N 0713858.1288$ $\begin{array}{lll}35 & 423500 N O 713838.1 & 288 \\ 36 & 423314 N 0713734.1 & 230\end{array}$

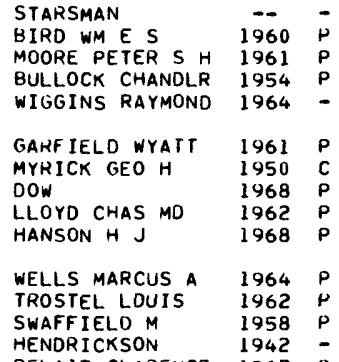

SLUNGWHITE S 1965 -

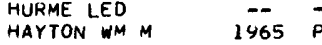

CHAPMAN KENNETH $1966-$

SLONGWHITE A

1957

JENDZA PETEK

MEDLA CHRIS

MILLER IVY

ALBERT GEO V JR 1965 P

8IANCHI DAVID A 1970 P

LISTOVICH GS $1964 P$

$\begin{array}{lll}\text { LYNCH THDMAS F } & 1959 & \text { P } \\ \text { GIHSON R I } & 1956-\end{array}$

$\begin{array}{lll}\text { RUSSELL HER8EKT } & 1968 & \text { A } \\ \text { NELSDN KOBERT } & 1958\end{array}$

LOYSEN D W

OUALLY

RIBEIRO J S S 1947

CADWELL OLIVE D $1 \overline{964}$

LEOM ST FOREST 1965 P

$\begin{array}{ll}\text { MDPW } & 1957 \\ \text { ARL IN KARL M } & 1967\end{array}$
BELAIR CLARENCE 1967 P

SPANACH N S $1967=$

$\begin{array}{lllll}6 & x & 205 & w & 16 \\ 6 & x & 190 & w & \\ 6 & x & 385 & w & \\ 6 & x & 215 & w & 16 \\ 6 & x & 150 & w & 56 \\ 6 & x & 207 & w & 37 \\ 6 & x & 153 & w & 21 \\ 6 & x & 170 & w & 28 \\ 6 & x & 110 & w & 1 \\ 6 & x & 245 & w & 131\end{array}$

$245 W$

$\begin{array}{rrrr}215 & 136 & =- & 69\end{array}$

$\begin{array}{lllll}70 & w & 44 & -- & 33 \\ 75 & w & -- & -- & --\end{array}$

126 W $36 \quad--24$

92

85
245

245

100 W $63 \quad-\quad 145$

28

230

170
380

$\begin{array}{rrr}12 & -- & 162 \\ 40 & = & 30\end{array}$

160

225

150
220

162

90

90
100
147

147

305

RUTLAND

SHIRLEY VILLAGE

SHIRLEY VILLAGE

FORT DEVENS

INDUST SCHOOL

DAW8DRN ALICE C 1965 P

MADDEN WM J JR

HURD LEON $H$

1964

$\begin{array}{ll}\text { HALLETT ROBT } & 1970 \\ & \end{array}$

GREGG PETER $r$

DROBISH A P

1969

BURSON JAMES

CLEVELAND MARY

PALSTON PAUL

BEQUN ROBERT

EVANS ELSIE

LANGLEY MELVIN

SHIRLEY VILLAGF

SHIRLEY VILLAGE

SHIRLEY VILLAGE

SHIRLEY VILLAGE

SHIRLEY VILLAGE

SHIRLEY VILLAGE

SHIRLEY VILLAG

SHIRLEY VILLAGE

SHIRLEY VILLAGE

SHIRLEY VILLAGE 1971 -

SHIRLEY VILLAGE 1971 -

SHIRLEY VILLAGF. 1971

SHIRLEY VILLAGE 1971

SHIRLEY VILLAGE 1971

1
6
6

24
350
200

190

SHIRLEY

$\begin{array}{rr}202 & \\ 12 & 0 \\ 2 & \\ -6 & x \\ 6 & x \\ 0 & x \\ 6 & x \\ 6 & x \\ 6 & x \\ 28 & x \\ 12 & x \\ 8 & x \\ -- & - \\ -- & x \\ -- & - \\ -- & - \\ -- & - \\ -2 & 0 \\ 2 & \\ 2 & 0 \\ 2 & 0 \\ 2 & 0 \\ 2 & 0 \\ 2 & 0 \\ 2 & 0 \\ 2 & 5 \\ 2 & 5 \\ 2 & 5 \\ 2 & 5 \\ 2 & 0 \\ 2 & 0 \\ 2 & 0 \\ 2 & 0 \\ 2 & 5 \\ & \end{array}$

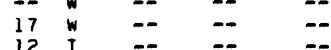

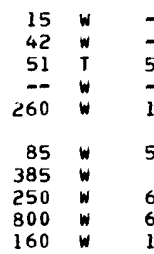

$\begin{array}{rllll}22 & w & -- & - & 11\end{array}$

$105 w \quad 8 \quad--10$

$100 w \quad--\quad=$

$\begin{array}{lllll}250 & w & = & = & = \\ 180 & w & = & = & = \\ 17 & w & -- & - & =\end{array}$

$\begin{array}{lllll}51 & 1 & -- & -- & --\end{array}$

$\begin{array}{lllll}17 & 1 & = & = & = \\ 18 & 1 & -z & = & =\end{array}$

$\begin{array}{lllll}13 & 1 & - & - & -\end{array}$

$\begin{array}{rrrr}8 & T & -- & - \\ 28 & T & - & R \\ 26 & T & - & K \\ 28 & T & -- & H \\ 35 & T & -- & R\end{array}$

--
2
3
5
4

$\begin{array}{ll}8-71 & 0 \\ 8-71 & 0 \\ 8-71 & 4 \\ 8-71 & 0\end{array}$

$\begin{array}{llll}25 & T & -- & -- \\ 30 & T & -- & - \\ 16 & T & -- & - \\ 14 & T & -- & -\end{array}$

翌

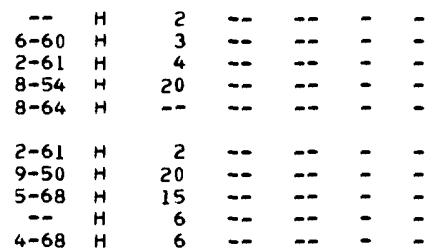

$12-64$
$6-62$

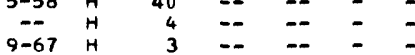

$\begin{array}{llllll}6-65 & \mathrm{H} & 3 & -- & -- & -\end{array}$

$\begin{array}{ccccccc}-- & H & -- & -- & = & = & = \\ 4-66 & H & H & 2 & -- & = & =\end{array}$

$\begin{array}{ccccccc}-- & H & - & -- & -- & - & P \\ 5-65 & H & 12 & = & = & = & \bar{P}\end{array}$

$\begin{array}{lllllll}5-65 & H & - & - & - & - & P \\ 2-71 & H & 15 & =- & -- & = & - \\ 6-70 & H & 15 & -- & -- & =\end{array}$

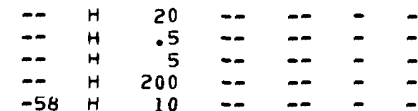

$\begin{array}{llllllll}- & H & H & 2 & -- & - & - & -\end{array}$

$\begin{array}{llllllll}-- & - & H & 3 & -- & -- & - & P \\ -- & 11-64 & H & 5 & -- & -- & - & P \\ & -- & H & -- & -- & -- & - & P\end{array}$

F $6-65 P \quad 40 \quad \ldots \quad \ldots \quad-$. 

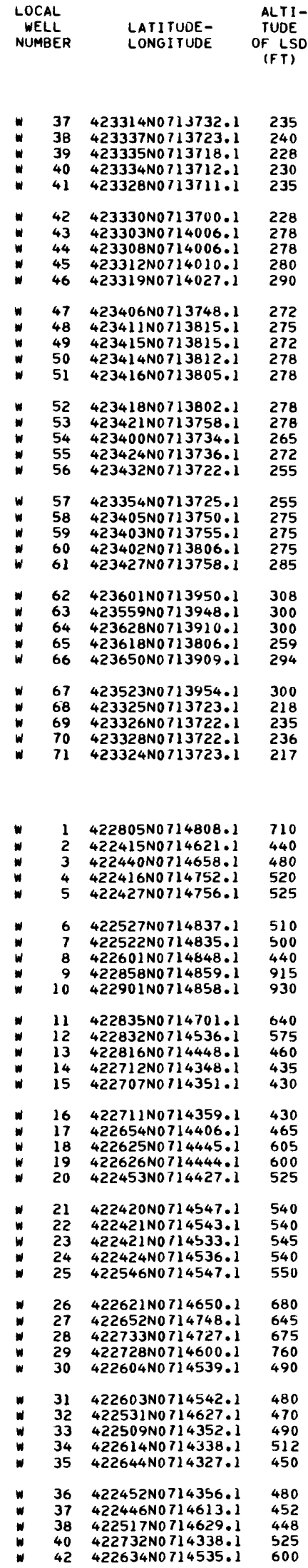

OWNER OR USER METHOU ḊIAM-IATIN-IDEPTHIUSE FEET WATERMETHOU DIAM-IFIN-IDEPTHIUSE TO BEARING LEVEL INATE IUSE ỸIELOI DO ITIME LOG OW

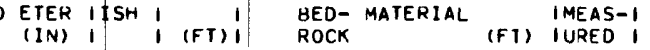
(GPM) Í(FT) Í(HR) SHIRLEY - CONTINUED

\begin{tabular}{|c|c|c|c|c|c|c|c|c|c|c|c|c|c|c|c|}
\hline $\begin{array}{l}\text { IIRLEY VILLAGE } \\
\text { IRLEY VILLAGE } \\
\text { IRLEE VILLAGE } \\
\text { IRLEY VILLAGE } \\
\text { IRLEEY VILLAGE }\end{array}$ & $\begin{array}{l}1972 \\
1971 \\
1971 \\
1971 \\
1971\end{array}$ & $\begin{array}{l}\overline{-} \\
\overline{-} \\
\overline{-}\end{array}$ & $\begin{array}{l}8 \\
2 \\
2 \\
2 \\
2\end{array}$ & $\begin{array}{l}\text { S } \\
0 \\
\text { S } \\
\text { S } \\
\text { S }\end{array}$ & $\begin{array}{l}40 \\
25 \\
43 \\
37 \\
39\end{array}$ & $\begin{array}{l}\mathbf{T} \\
\mathbf{T} \\
\mathbf{T} \\
\mathbf{T} \\
\mathbf{T}\end{array}$ & $\begin{array}{l}-- \\
-- \\
37 \\
--\end{array}$ & $\begin{array}{r}B R \\
-- \\
8 R \\
R \\
7 R\end{array}$ & $\begin{array}{l}7 \\
5 \\
6 \\
5 \\
6\end{array}$ & $\begin{array}{l}10-71 \\
10-71 \\
10-71 \\
10-71 \\
10-71\end{array}$ & $\begin{array}{l}u \\
u \\
u \\
u \\
u\end{array}$ & $\begin{array}{r}125 \\
-- \\
50 \\
15 \\
10\end{array}$ & $\begin{array}{l}18 \\
-- \\
=- \\
--\end{array}$ & $\begin{array}{l}168 \\
=- \\
=- \\
=-\end{array}$ & $\begin{array}{l}D \\
D \\
D \\
D \\
D\end{array}$ \\
\hline $\begin{array}{l}\text { IRLEY VILLAGE } \\
\text { IRLEY VILLAGE } \\
\text { IRLEY VILLAGE } \\
\text { IRLEY VILLAGE } \\
\text { IRLEY VILLAGE }\end{array}$ & $\begin{array}{l}1971 \\
1971 \\
1971 \\
1971 \\
1971\end{array}$ & $\begin{array}{l}\bar{z} \\
\overline{-} \\
-\end{array}$ & $\begin{array}{l}2 \\
2 \\
2 \\
2 \\
2\end{array}$ & $\begin{array}{l}\mathrm{S} \\
0 \\
\mathrm{~S} \\
0 \\
\mathrm{~S}\end{array}$ & $\begin{array}{l}35 \\
34 \\
36 \\
40 \\
29\end{array}$ & $\begin{array}{l}1 \\
T \\
T \\
T \\
T\end{array}$ & $\begin{array}{l}=- \\
=- \\
=- \\
=-\end{array}$ & $\begin{array}{l}8 K \\
7 R \\
8 K \\
-\bar{T} \\
T\end{array}$ & $\begin{array}{r}9 \\
5 \\
2 \\
-5 \\
7\end{array}$ & $\begin{array}{l}10-71 \\
10-71 \\
10-71 \\
=- \\
10-71\end{array}$ & $\begin{array}{l}u \\
u \\
u \\
u \\
u\end{array}$ & $\begin{array}{l}12 \\
10 \\
25 \\
--3\end{array}$ & $\begin{array}{l}=- \\
=- \\
=-\end{array}$ & $\begin{array}{l}=- \\
=- \\
=-\end{array}$ & $\begin{array}{l}D \\
D \\
D \\
D \\
D\end{array}$ \\
\hline $\begin{array}{l}\text { BERT JOHN A } \\
\text { RNSWORTH RAY } \\
\text { OMPSON D } \\
\text { LLIS CARLTON } \\
\text { GHES DANIEL F }\end{array}$ & $\begin{array}{l}1951 \\
1957 \\
-- \\
1967 \\
1967\end{array}$ & $\begin{array}{l}v \\
0 \\
v \\
- \\
-\end{array}$ & $\begin{array}{r}1 \\
36 \\
3 \\
-- \\
--\end{array}$ & $\begin{array}{l}\text { S } \\
0 \\
\text { T } \\
- \\
-\end{array}$ & $\begin{array}{l}22 \\
14 \\
15 \\
32 \\
20\end{array}$ & $w$ & $\begin{array}{l}=- \\
=- \\
=-\end{array}$ & $\begin{array}{l}K \\
T \\
S \\
R \\
S\end{array}$ & $\begin{array}{c}-- \\
8 \\
-- \\
--\end{array}$ & $\begin{array}{c}-- \\
6-73 \\
=- \\
=-\end{array}$ & $\vec{H}$ & $\begin{array}{l}-- \\
-- \\
30 \\
--\end{array}$ & $\begin{array}{l}=- \\
=- \\
=- \\
--\end{array}$ & $\begin{array}{l}=- \\
=- \\
=-\end{array}$ & $\begin{array}{l}- \\
- \\
-\end{array}$ \\
\hline $\begin{array}{l}\text { MP PHILIPO P } \\
\text { RO ERNES } \\
\text { HWECKENDIECK } \\
\text { OD JAMES A } \\
\text { LLOWELL HENRY }\end{array}$ & $\begin{array}{l}1967 \\
1957 \\
1970 \\
-- \\
1950\end{array}$ & $\begin{array}{l}v \\
o \\
v \\
- \\
-\end{array}$ & $\begin{array}{l}-- \\
-- \\
10 \\
--\end{array}$ & $\begin{array}{l}\overline{0} \\
0 \\
- \\
-\end{array}$ & $\begin{array}{r}40 \\
5 \\
50 \\
89 \\
60\end{array}$ & $w$ & $\begin{array}{l}=- \\
=- \\
=-\end{array}$ & $\begin{array}{r}H \\
S \\
R \\
- \\
S\end{array}$ & $\begin{array}{r}-- \\
2 \\
10 \\
15 \\
8\end{array}$ & $\begin{array}{r}-- \\
-57 \\
7-70 \\
-69 \\
11-63\end{array}$ & $\underset{H}{H}$ & $\begin{array}{l}-- \\
\overline{32} \\
\overline{20}\end{array}$ & $\begin{array}{l}=- \\
=- \\
=- \\
=\end{array}$ & $\begin{array}{l}=- \\
=- \\
=- \\
--\end{array}$ & $\begin{array}{l}- \\
\overline{-} \\
\overline{-}\end{array}$ \\
\hline $\begin{array}{l}\text { YLOR DANIEL H } \\
\text { LMARTIN JOHN } \\
\text { NDRON ROBERT } \\
\text { OK GLADYS T } \\
\text { OK GL MICHAEL } 8\end{array}$ & $\begin{array}{l}1971 \\
1955 \\
1965 \\
--\end{array}$ & $\begin{array}{l}\bar{v} \\
v \\
\bar{o}\end{array}$ & $\begin{array}{r}-- \\
1 \\
-\frac{1}{30}\end{array}$ & $\begin{array}{l}\dot{s} \\
\text { I } \\
\bar{w}\end{array}$ & $\begin{array}{r}300 \\
30 \\
20 \\
-- \\
15\end{array}$ & & $\begin{array}{l}=- \\
=- \\
=- \\
=-\end{array}$ & $\begin{array}{r}-- \\
R \\
K \\
-S\end{array}$ & $\begin{array}{l}-- \\
10 \\
10 \\
--\end{array}$ & $\begin{array}{l}-- \\
-72 \\
-73 \\
-- \\
-72\end{array}$ & 4 & $\begin{array}{r}-- \\
1 \\
5 \\
--\end{array}$ & $\begin{array}{l}=- \\
=- \\
=- \\
=\end{array}$ & $\begin{array}{l}=- \\
=- \\
=-\end{array}$ & $\begin{array}{l}- \\
\overline{-} \\
\overline{-}\end{array}$ \\
\hline $\begin{array}{l}\text { ERICOLA ROBERT } \\
\text { HELPS JESSE J } \\
\text { AINES WILLIAM } \\
\text { OUTENIS S } \\
\text { EANNOTTE ROY L }\end{array}$ & $\begin{array}{l}1969 \\
1968 \\
1964 \\
1970 \\
1972\end{array}$ & $\begin{array}{l}w \\
- \\
k \\
v \\
v\end{array}$ & $\begin{array}{r}-- \\
-2 \\
8 \\
6\end{array}$ & $\begin{array}{l}5 \\
5 \\
5 \\
0 \\
5\end{array}$ & $\begin{array}{l}15 \\
45 \\
21 \\
20 \\
65\end{array}$ & & $\begin{array}{l}-- \\
22 \\
-- \\
--\end{array}$ & $\begin{array}{l}R \\
R \\
R \\
R \\
S\end{array}$ & $\begin{array}{l}14 \\
20 \\
-8\end{array}$ & $\begin{array}{l}-= \\
7-64 \\
=-72 \\
6-72\end{array}$ & $\mathrm{H}$ & $\begin{array}{l}9 \\
-23 \\
-- \\
10\end{array}$ & $\begin{array}{l}=- \\
=- \\
=-\end{array}$ & $\begin{array}{l}=- \\
=- \\
=- \\
--\end{array}$ & $\begin{array}{l}- \\
\overline{-} \\
\overline{-}\end{array}$ \\
\hline $\begin{array}{l}\text { EELER } \\
\text { IRLEY VHSTER } \\
\text { IRLEY VILLAGE } \\
\text { IRLEY VILLAGE } \\
\text { IRLEY VILLAGE }\end{array}$ & $\begin{array}{l}1941 \\
1973 \\
1973 \\
1973 \\
1973\end{array}$ & $\frac{0}{w}$ & $\begin{array}{r}30 \\
8 \\
2 \\
2 \\
2\end{array}$ & $\begin{array}{l}w \\
\text { s } \\
\text { S } \\
\text { s } \\
\text { s }\end{array}$ & $\begin{array}{l}10 \\
52 \\
62 \\
75 \\
49\end{array}$ & T & $\begin{array}{l}-- \\
52 \\
62 \\
75 \\
49\end{array}$ & $\begin{array}{l}K \\
R \\
R \\
R \\
R \\
R\end{array}$ & $\begin{array}{r}6 \\
6 \\
17 \\
65 \\
3\end{array}$ & $\begin{array}{r}7-73 \\
10-73 \\
8-73 \\
10-73 \\
9-73\end{array}$ & 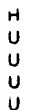 & $\begin{array}{r}-- \\
240 \\
-- \\
--\end{array}$ & $\begin{array}{l}-- \\
16 \\
-- \\
--\end{array}$ & $\begin{array}{l}-- \\
24 \\
-- \\
--\end{array}$ & $\begin{array}{l}\bar{D} \\
\bar{D} \\
\bar{D}\end{array}$ \\
\hline
\end{tabular}

STERLING

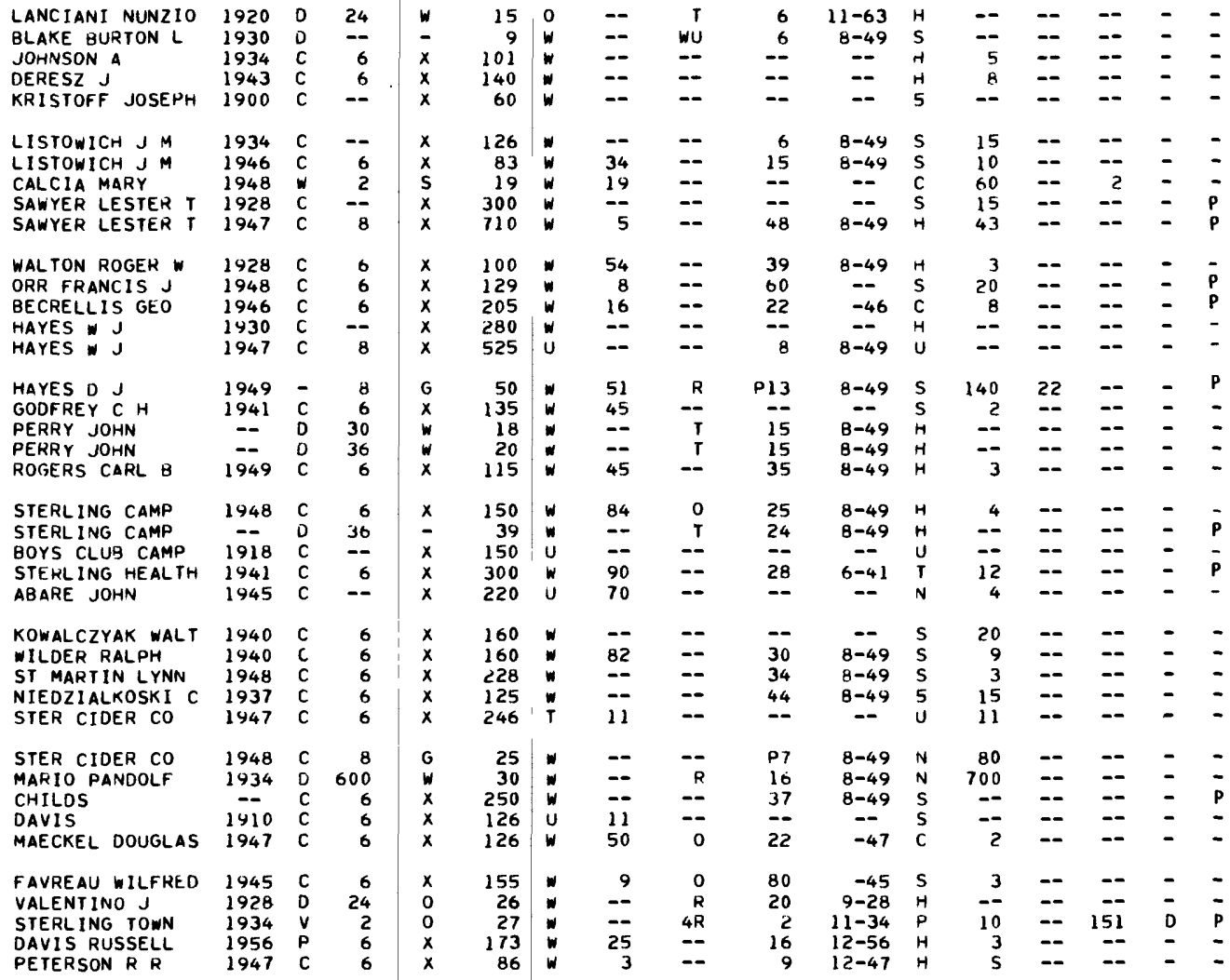


LOCAL

WELL ALTITI LATITUDE- TUDE
LONGITUDE OF LSD (FT)

OWNER OR USER YEAK/ METHOD DIAM-IFIN-IDEPTHIUSE FEET WATERDRILLED ETER IISH I । (IN) I ISH IFT) GEARING STERLING --CONTINUED

1. $43422525 N 0714747.1440$ $44 \quad 422623 N 0714446.1 \quad 610$ $422850 N 0714600.1 \quad 530$ $422444 \mathrm{NO} 714804.1 \quad 490$

$51422457 N 0114438.1445$ $\begin{array}{lll}52 & 422518 N 0714627.1 & 440 \\ 53 & 422510 N 0714400.1 & 490\end{array}$ $\begin{array}{lll}53 & 422510 N 0714400.1 & 490 \\ 54 & 422451 N 0714708.1 & 418\end{array}$ 55 422749N0714631.1

$56422843 \mathrm{~N} 0714851.1$ $57422433 \mathrm{NO} 714518.1$ $58422604 N 0714850.1$ 60422840 NOT14634.1

$61422537 \mathrm{~N} 0714754.1$ $62422447 \mathrm{NO} 714402$ 63 422503N0714354.1

$64 \quad 422745 N 0714452.1$

$66422756 \mathrm{~N} 0714455.1$ $67422718 N 0714458$. 1 $68422715 N 0714450.1$ $69422452 \mathrm{NO} / 14450.1$

$71422444 N 0714435.1$ 72422436 NOT14436.1 $73422425 N 0714446.1$

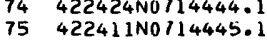

$76422804 N 0714508.1$ $77422802 N 0714503.1$ $78 \quad 422837 N 0714649.1$ 89422745 N0714726.1

B1 422642N0714752.1 82422639 N0714727.1 $83422725 N 0714629.1$ $84422649 N 0714629.1$ 45 422638N0714535.1

$86 \quad 422548 N 0714546.1$ 87422445 N0714523.1 88 422444NOT14515. 99 422443N0714517.

$91422404 N 0714616.1$ 92 422418N0714007.1 $93422413 N 0714752$. 94 422449N0714806.1

96422452 NO 114754.1 $97422539 N 0714840.1$ 98 422543N0714915.1 99 422556N0714908.1

W 100 422602N0714951.1

w 101 422617N0714918.1 - $102422522 N 0714637.1$ - 103422523 N0713634.1 104 422525N0714628.1 105 422523N0114631.1

W $106 \quad 422522 N 0714637.2$ $107422525 N 0714632.1$ $108422525 N 0714632.2$ W 110 422631N0114846.1

w 111422827 N0114448.2 $112422824 N 0714443.1$ $113422824 N 0714452.1$ 114
115
1

- 116422858 N0714636.1 714 - 117422840 NO114642.1 005 w 118 422716N0714745.1 551

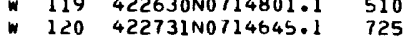

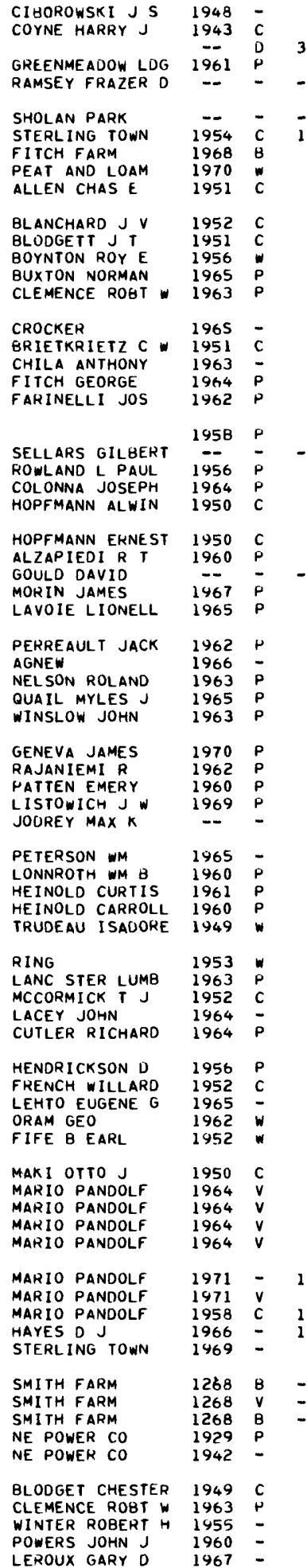

\begin{tabular}{|c|c|c|c|c|c|c|c|c|c|c|c|c|c|}
\hline $\begin{array}{l}1 \\
6\end{array}$ & $\begin{array}{l}s \\
x\end{array}$ & $\begin{array}{r}42 \\
108\end{array}$ & $\bar{z}$ & $\because$ & $u$ & 10 & $7-48$ & - & 6 & $=$ & 3 & D & $\begin{array}{l}P \\
-\end{array}$ \\
\hline 36 & $\hat{w}$ & 15 & u & - & $=$ & 9 & $5-39$ & $\hat{u}$ & - & - & $=$ & - & $=$ \\
\hline 6 & $x$ & 190 & $w$ & 98 & -- & -- & $\ldots$ & c & 8 & -- & -- & 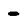 & P \\
\hline-- & - & 96 & $\omega$ & $=$ & - & $=$ & - & H & -- & - & - & - & P \\
\hline$\cdots$ & - & -- & $w$ & - & - & -- & - & $\mathbf{R}$ & - & -- & - & $=$ & P \\
\hline 12 & G & 31 & $w$ & - & $B R$ & 4 & $3-54$ & P & 410 & 16 & 196 & D & P \\
\hline 2 & $x$ & 27 & $T$ & 27 & $=$ & - & - & $u$ & - & $=$ & 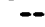 & D & - \\
\hline 2 & S & 52 & $W$ & -- & $4 R$ & 4 & $1-70$ & $N$ & 65 & 2 & 2 & - & - \\
\hline 6 & $x$ & 123 & $\omega$ & 22 & -- & 29 & $6-51$ & $\mathrm{H}$ & 5 & -- & - & - & - \\
\hline 6 & $x$ & 140 & $w$ & 2 & - & 40 & $2-52$ & $H$ & 6 & -. & - & - & 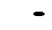 \\
\hline 6 & $x$ & 95 & $w$ & 33 & -- & $F$ & $7-51$ & H & 2 & -- & -- & - & - \\
\hline 2 & S & 35 & $w$ & - & $R$ & - & -- & H & 8 & - & - & D & - \\
\hline 6 & $x$ & 40 & $w$ & 11 & - & 16 & $7-65$ & $H$ & 30 & -- & -- & $=$ & 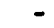 \\
\hline 6 & $x$ & 310 & $w$ & 7 & -- & 20 & $10-63$ & $H$ & 3 & -- & -- & - & - \\
\hline 6 & $x$ & 110 & $w$ & 105 & - & 110 & $7-65$ & $H$ & - & -- & - & - & 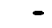 \\
\hline 6 & $x$ & 170 & $\omega$ & 11 & -- & 22 & $10-51$ & $H$ & 2 & -- & - & - & - \\
\hline 6 & $x$ & 206 & $\omega$ & 15 & - & 44 & $10-63$ & H & - & -- & - & - & - \\
\hline 6 & $x$ & 115 & $w$ & 5 & -- & 22 & $8-64$ & H & 10 & $\cdots$ & - & - & - \\
\hline 6 & $x$ & 170 & $w$ & 80 & -- & $=$ & - & $H$ & 15 & -- & -- & - & - \\
\hline 6 & $x$ & 320 & $w$ & 87 & -- & 59 & $3-58$ & H & 7 & - & - & - & 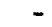 \\
\hline-- & - & 205 & $W$ & - & $=$ & - & $=$ & $H$ & - & - & - & - & $\mathbf{P}$ \\
\hline 6 & $x$ & 190 & $w$ & 41 & -- & 15 & $5-56$ & $H$ & 4 & -- & - & - & - \\
\hline 6 & $x$ & 150 & $w$ & 89 & - & -- & -- & $H$ & 2 & -- & -- & - & - \\
\hline 6 & $x$ & 237 & 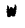 & 33 & -- & 10 & $7-50$ & H & 4 & $\cdots$ & - & - & - \\
\hline 6 & $x$ & 222 & $w$ & 39 & -- & 6 & $7-50$ & H & 4 & - & -- & - & - \\
\hline 6 & $x$ & 580 & $w$ & 97 & -- & -- & - & $P$ & .5 & -- & - & 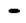 & - \\
\hline-- & $=$ & 92 & $w$ & - & U & $\cdots$ & $\cdots$ & $H$ & - & - & -- & - & 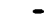 \\
\hline 6 & $x$ & 325 & $w$ & 106 & -- & $F$ & $11-67$ & $H$ & 2 & - & $\cdots$ & - & - \\
\hline 6 & $x$ & 200 & $\omega$ & 89 & -- & -- & -- & $H$ & 4 & -- & $=-$ & - & - \\
\hline 6 & $x$ & 375 & $w$ & 57 & -- & 36 & $6-62$ & $H$ & 20 & -- & -- & - & - \\
\hline 6 & $x$ & 145 & $\omega$ & 105 & -- & 15 & $4-66$ & $H$ & 15 & -- & -- & - & 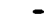 \\
\hline 6 & $x$ & 100 & $w$ & 33 & - & 30 & $9-63$ & $H$ & 35 & -- & - & - & . \\
\hline 6 & $x$ & 50 & $w$ & 8 & -- & -- & -- & $H$ & 6 & -- & -- & - & 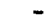 \\
\hline 6 & $x$ & 345 & $w$ & 61 & -- & 21 & $9-63$ & H & 2 & - & - & - & - \\
\hline 6 & $x$ & 290 & $W$ & 95 & 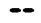 & 20 & $2-70$ & $H$ & 4 & -- & - & - & - \\
\hline 6 & $x$ & 160 & $w$ & 42 & - & 7 & $6-62$ & H & 6 & - & -- & - & - \\
\hline 6 & $x$ & 170 & $\omega$ & 94 & -- & 25 & -60 & H & 6 & - & -- & - & 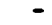 \\
\hline 6 & $x$ & 245 & $W$ & 103 & -- & 25 & $6-69$ & H & 4 & - & - & - & $=$ \\
\hline 1 & $x$ & 120 & $\omega$ & -- & - & -- & -- & $H$ & $=-$ & -- & $\cdots$ & - & P \\
\hline 6 & $x$ & 230 & $W$ & 135 & - & 30 & $11-65$ & $H$ & 2 & -- & - & - & - \\
\hline 6 & $x$ & 115 & $w$ & 64 & -- & 9 & $3-60$ & $H$ & 6 & - & - & - & - \\
\hline 6 & $x$ & 216 & $W$ & 62 & -- & $=$ & $\cdots$ & H & 2 & - & $\cdots$ & - & - \\
\hline 6 & $x$ & 327 & $w$ & 67 & - & 50 & $3-60$ & $H$ & .5 & -- & $\cdots$ & - & 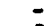 \\
\hline 2 & s & 34 & $w$ & -- & $u$ & 6 & $10-49$ & $H$ & 5 & -- & 1 & - & $\mathbf{P}$ \\
\hline 2 & $P$ & 18 & $w$ & - & $R$ & 4 & $3-53$ & $H$ & 28 & -- & -- & D & - \\
\hline 6 & $x$ & 110 & 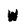 & 38 & - & - & - & $H$ & 5 & - & - & - & - \\
\hline 6 & $x$ & 109 & $w$ & 16 & - & 12 & $10-52$ & $H$ & 5 & -- & - & - & - \\
\hline 6 & $x$ & 163 & $w$ & 50 & - & - & - & $H$ & 8 & - & - & - & - \\
\hline 6 & $x$ & 225 & $w$ & 18 & - & -- & - & $H$ & 5 & -- & $=$ & - & - \\
\hline 6 & $x$ & 102 & $m$ & 28 & - & 14 & $12-56$ & $H$ & 2 & -- & -- & - & - \\
\hline 6 & $x$ & 160 & $w$ & 6 & -- & 5 & $8-52$ & $H$ & 2 & - & -- & - & - \\
\hline 6 & $x$ & 204 & $w$ & 19 & - & -- & -- & $H$ & 5 & -- & 2 & - & - \\
\hline 2 & $P$ & 32 & 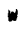 & -- & $R$ & 9 & $10-62$ & $H$ & 4 & $\cdots$ & $\cdots$ & D & - \\
\hline 2 & S & 25 & $w$ & - & $u$ & 15 & $9-52$ & $H$ & 25 & -- & -- & - & - \\
\hline 6 & $x$ & 66 & $w$ & 18 & -- & 18 & $10-50$ & H & 5 & -- & -- & - & - \\
\hline 2 & $\mathbf{S}$ & 42 & $T$ & 48 & $R$ & 9 & $9-64$ & $u$ & 45 & 4 & 2 & D & - \\
\hline 2 & $\mathbf{S}$ & 43 & $T$ & 43 & $R$ & 7 & $9-64$ & $u$ & 45 & - & 2 & D & - \\
\hline 2 & S & 33 & $\mathrm{~T}$ & - & R & 15 & $9-64$ & $u$ & 20 & -- & - & D & - \\
\hline 2 & 5 & 36 & $T$ & -- & $R$ & 10 & $9-64$ & u & 35 & -- & -- & D & - \\
\hline 12 & G & 45 & $w$ & - & R & 4 & $3-71$ & $N$ & 200 & - & -- & 0 & - \\
\hline 2 & $S$ & 39 & $T$ & -- & $R$ & 7 & $4-71$ & $u$ & 10 & -- & -- & D & - \\
\hline 12 & G & 32 & $w$ & 32 & R & 13 & $4-58$ & $\mathbf{N}$ & 318 & 7 & 12 & D & - \\
\hline 12 & G & 37 & $w$ & 56 & $R$ & 3 & $3-66$ & $\mathrm{~s}$ & 250 & - & - & - & - \\
\hline 8 & s & 75 & $\mathbf{T}$ & - & $B R$ & 4 & $10-69$ & $u$ & 500 & 6 & 49 & D & - \\
\hline- & $x$ & 7 & $T$ & -- & $\mathbf{T}$ & -- & - & $u$ & -- & -- & - & D & - \\
\hline- & 0 & 19 & $T$ & $=$ & $\mathbf{T}$ & 2 & $2-68$ & $u$ & -- & - & - & D & 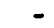 \\
\hline- & $x$ & 24 & $T$ & 24 & $\boldsymbol{T}$ & 4 & $2-68$ & $u$ & - & -- & -- & D & - \\
\hline 0 & $x$ & 190 & $u$ & 40 & - & 97 & $6-65$ & $u$ & 3 & 73 & 5 & - & - \\
\hline 8 & $x$ & 362 & $w$ & 56 & -- & 68 & $6-62$ & $H$ & 4 & - & - & D & - \\
\hline 6 & $x$ & 204 & $w$ & 0 & -- & 27 & $5-49$ & $H$ & 2 & - & -- & - & - \\
\hline 6 & $x$ & 310 & $w$ & 7 & - & 20 & $10-63$ & H & 3 & -- & -- & & \\
\hline 6 & - & 155 & $w$ & 20 & - & 6 & -55 & $H$ & 3 & - & $\cdots$ & - & - \\
\hline 6 & $x$ & 107 & $w$ & 40 & - & 2 & -60 & H & 20 & - & - & - & - \\
\hline 6 & $x$ & 180 & $w$ & 91 & - & - & - & H & 7 & - & - & . & \\
\hline
\end{tabular}


TABLE 1.--DESCRIPTIDN OF SELECTEO WELLS, TEST WELLS, AND BORINGS -- CONTINUED

$\begin{array}{lll}\text { LOCAL } & \text { ALTI- } \\ \text { WELL } & \text { LATITUDE- } & \text { TUDE } \\ \text { NUMBER } & \text { LONGITUDE } & \text { OF LSO }\end{array}$

WULL

$$
\text { (FT) }
$$

1423939 N0714213.1 $\begin{array}{ll}2 & 424018 N 0714221.1 \\ 3 & 424035 N 0714421.1\end{array}$ $\begin{array}{ll}3 & 424035 N 0714421.1 \\ 4 & 423955 N 0714228.1\end{array}$ $\begin{array}{ll}4 & 423955 N 0714228.1 \\ 5 & 424021 N 0714203.1\end{array}$

$6424021 N 0714200.1$ 7
8 $424030 \mathrm{~N} 0714116.1$ 8
9 $424030 N 0714116.2$ 11 424012N0714250.1

$12424006 \mathrm{~N} 0714220.1$ $13424055 N 0714353.1$ is 424019N0714556.1 16 423902NO113934.1

17 424033N0714626.1 18 424019N0714554.1 $19424047 N 0714548.1$ $\begin{array}{ll}20 & 423754 N 0714359.1 \\ 21 & 423806 N 0714351.1\end{array}$

22 423810N0714411.1 $23423836 N 0714437$. 24 423846NO714440.

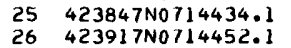

27 423925N0714422.1

28 423925N0714334.1

30
31 423830 NO714341.1

32 423747N0714113.1

33 423924N0714007.1

$34 \quad 423953 N 0714017.1$

36 424101N0714037.1

37 424112N0714037.1

$3 B \quad 424129 N 0714037$.

$424043 N 0714331$.

40
41 $424051 N 0714259.1$

$42423852 \mathrm{~N} 0714030.1$

$43424014 N 0714118.1$

44
45 $424136 N 0714049.1$

45 424152N0713958.1
$46 \quad 424133 N 0714516.1$

47 424143N0714544.1

$48 \quad 424215 N 0714338.1$

49 424048N0714331.

$51424044 \mathrm{NO} / 14220.1$

$52424041 N 0714206.1$

53 424202N0713954.

54 424208N0713956.

56 423942NO714002.1

$57424022 N 0713915.1$

58 424016NO113927.

$60424044 N 0714126.1$

61 424035NO/14150.1

b2 423848N0714613.1

$63423922 N 0714320$.

64 423854N0714349.1

65
66 423850 N 2714410.1

$67423833 N 0714231.1$

68 423856N0714119.

$69423754 N 0714118.1$

$70424035 N 0714515$.
270
310

330

295

290

335

335
315
315

308
310

310
440

440
470
275

530

530
965
340

340
468

478

535
625

625
648

640
615

622
512

325

325
485
472

350
280

395

560
550

532

470
290

305
322

285

310
360

360
482

325

340
690

308

325

480

410

348

308
330

305
310

310
298

468
500

518
575

355

360
280

280
361
315

315
275

$\begin{array}{lcc}\text { UAGHINI JOHN R } & 1968 & - \\ \text { WHITMAN B } & 1972 & R \\ \text { HUL ICK H } & 1955 & R \\ \text { LAFAYETTE LE E } & -- & - \\ \text { STERLING TOWN } & 1963 & B \\ & & \\ \text { STERLING TOWN } & 1963 & \text { G } \\ \text { STERLING TOWN } & 1963 & B\end{array}$

FESSENOEN CO WT TOWNSEND SCH
FESSENDEN CO FESSENDEN

ADAMS U S
SHERRIN MARY

SHERRIN MARY

DDLE A

$\begin{array}{ll}\text { EDWARDS JDHN } & 1890 \\ \text { SPAULDING MEM S } & 1931\end{array}$

TOWNSEND TOWN 1886

US GEOL SURVEY 1964

PEARL HILL BRK

N MIDDLESEX HS 1960

HAGSTROM ROBT N 1960 P

CONNORS WILLIAM 1965 -

ETANI KENJI 1969 P

BOURQUE RAYMOND 1964

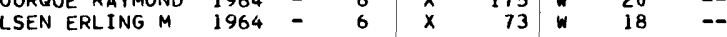

HARTMANN M

ANNULA

WILSON DONALD 1964

MAKI MARTIN

1965

POUDRIER JOS

PANANOS ANGELO

SPAULDING WM 1970

AHO VILJO
AHO ARVO

FDRD CLIFFORD

LEWIS BURTON

SULLIVAN $R P$

OJALA JORMI L

1966

$1964=$
$1964=$

PACKARD WALTER

AHO SELMA

BEESE WALTER T

TREEMAN GEORGE

SISSOM HAROLU

1965

1965

1965

ARCHAMBAULT L

WORNHAM AUBRE

HOOPER ROBT

LARSON LEE J

1966

IDE EDWARD L

1950
1965

1965
1961

ROBERTS HERBERT 1967 A

LOWE CLARENCE O 1966

$\begin{array}{ll}\text { ESPOSITO MARY B } & 1971 \\ \text { WARNER JAMES A } & 1971\end{array}$

WILSON GEORGE R 1964

PHINNEY PAUL T 1971

MAUMAN HERBORT 1967 D

MEAUREGARD FE 1950 - 24

\begin{tabular}{lcll} 
ROBERTS WILLIAM & -- & 0 & $\mathbf{3 0}$ \\
\hdashline-
\end{tabular}

$\begin{array}{llll}\text { GREELEY OLINT } & 1956 & - & 6 \\ \text { PERIGHY LIONEL } & 1964 & \overline{0} & 36\end{array}$

FRANCOEUR JAMES 1965 D 42

$\begin{array}{lccc} & -- & D & 48 \\ \text { LAITINEN PAUL } & 1966 & - & -- \\ \text { AALTO WAINO } & 1962 & D & 30 \\ \text { GOULD CHARLES } & 1937 & D & --\end{array}$

BECHARD WILFRED

LEAHY FRANK

TOWNSEND TOWN

HICKS R M INC

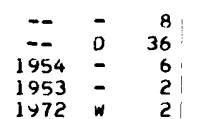

TOWNSEND

WELL- - FEET WATER(IN) I I I (FT)! ROCK

LEVE LEVEL IMATE
IMEAS(FT) IURES- I YIELOI DO ITIME LOG OW STERLING --CONTINUED

$\begin{array}{rrrrrrrrrrrrrr}6 & X & 208 & W & 175 & -- & -- & -- & H & 20 & -- & -- & - & - \\ 8 & X & 280 & W & 8 & -- & 8 & -72 & H & 5 & -- & -- & - & - \\ 4 & X & 88 & W & 3 & -- & 60 & -55 & H & 15 & -- & - & - & - \\ 6 & X & 100 & W & -- & -- & -- & - & H & -- & -- & -- & - & - \\ 2 & - & 15 & T & -- & -- & 0 & -63 & 0 & -- & -- & -- & D & - \\ 2 & - & 7 & T & -- & -- & 0 & -63 & 0 & -- & -- & -- & 0 & - \\ 2 & - & 10 & T & -- & -- & 0 & -63 & 0 & -- & -- & -- & D & -\end{array}$


TABLE 1.--DESCRIPTION OF SELECTED WELLS, TEST WELLS, AND BORINGS -- CONTINUEN

LOCAL WELL

LATITUDELONG I TUDE

ALTI-
TUDE OF LSO

OWNER OR USER METHOD DIAM-IFIN-IDEPTHTUSE TO BEARING \begin{tabular}{c|l|l} 
DRILLED ETER IISH I BED- MATERIAL \\
(IN) I I IFT) I ROCK
\end{tabular} (IN) I I (FT)

$72423842 N 0714055.1$ 73 424025N0714624.1 $74 \quad 424045 N 0714257.1$ 75 423828N0713958.1

1422116 N0714809.1 2
3 $422116 N 0714818.2$ 3 422324N0714731.1 $4 \quad 422125 N 0714808.1$
5

645 645
395
645

$6422312 N 0714752.1$

7 422224N0714818.1

8 422224N0714818. 10 4223221N0714903.1

$11422328 \mathrm{No} 714806.1$ $12422131 N 0714730.1$

$13422033 \mathrm{NO} 714611.1$

18 422141N0714833.

20 422335N0714626.1

20 422335N0714626.1

22 422052N0712319.

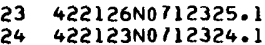

492

1423332 N0715556.1 1106 2 $423254 N 0715434.11009$ $3423239 \mathrm{N0715356.1} 930$ 2 423337N0715622.1 1170

3 423348N0715558.1 1160

$4 \quad 423458$ N0715522.1 892

$\begin{array}{rrr}5 & 423458 N 0715522.2 & 892 \\ 6 & 423458 N 0715522.3 & 892\end{array}$

$\begin{array}{rrr}6 & 423458 N 07115522.3 & 892 \\ 7 & 423310 N 0715519.1 & 1085\end{array}$

$8423302 N 0715436.1 \quad 1030$ 9423300 NOT15432.1 1035 $\begin{array}{lll}10 & 423258 N 0715435.1 & 1020 \\ 11 & 423402 N 0715418.1 & 1035\end{array}$ $12423449 N 0715412.1815$

$13423523 N 0715417.1$

14 423522NOI15400.1

15 423459NOr15406.1 805

$\begin{array}{lll}16 & 423451 \text { NO715410.1 } & 805 \\ 17 & 423403 N 0713246.1 & 760\end{array}$

18423400 NOT15247.1 745 $19423233 \mathrm{~N} 0715434.11022$ 20423049 N0715407.1 1150 $22423549 N 0715150.1 \quad 715$

$24 \quad 423247$ N0715448.1 1078 25 423233N0715433.1 1080 $\begin{array}{rrr}28 & 423130 N 0715242.1 & 905 \\ 30 & 423218 N 0715409.1 & 1050\end{array}$ $\begin{array}{lll}30 & 423218 N 0715409.1 & 1050 \\ 31 & 423148 N 0715515.1 & 1060\end{array}$

32423113 N0715454.1 1245 $36 \quad 423258$ No715310.1 860 $\begin{array}{lll}38 & 423229 N 0715619.1 & 1110 \\ 39 & 423230 N 0715622.1 & 1130\end{array}$ 40 423135NO715646.1 1110

42423457 N0715412.1 795 $43 \quad 423455 N 0715411.1790$ 44 423458NO715412. $\begin{array}{lll}45 & 423457 N 0715410.1 & 790 \\ 46 & 423501 \text { NO } 715413.1 & 795\end{array}$

$47 \quad 423502$ NOT15418.1 797 48 423502NO715417.1 787 $\begin{array}{lll}49 & 423059 N 0715323.1 & 900\end{array}$ 51423053 NO715323.1 898
HICKS R M INC ILLARO BROOK TOWNSEND TOWN TOWNSEND TOWN TOWNSEND TOWN

TOWNSEND TOWN

W BOYLSTON WD W BOYLSTON WD CHAPMAN ROBT E WOYLSTON WD WHITING ALFRED LEWIS 8 HAROLD MEYER WALTER

NORDSTROM B WACHUSETT CC AMER ICAN LEGION PIERPONT HARLAN

TOWNSEND - - CONT INUED

$\begin{array}{rrrrrrrr}1972 & - & 18 & G & 61 & W & 62 & R \\ -1975 & W & 6 & X & 188 & W & - & H \\ 1975 & W & 2 & 0 & 58 & T & -- & 25 \\ 1974 & W & 2 & 0 & 95 & T & -- & 25 \\ 1975 & W & 2 & & 63 & T & -- & 25\end{array}$

$1975 \mathrm{~W}$

WEST BOYLSTON

* BOYLSTON WD W BOYLSTON WD W BOYLSTON WD WOYLSTON WD W BOYLSTON WD MDC

\section{MOPW}

MDPW

HAYNES ENOS

KARKKAEN ANDRE

JAASKELAINEN H

GARDNER ST HOSP

GARDNER ST HOS

GARDNER ST HOSP
SORILA ARVO

LUOMA ELMER E

SAINI JACOB

KEYSER F A

WILEN A

ENGMAN $S$

ADAMS CHARLES

MIKI E

SABILAMPI BROS

$\begin{array}{lll}1940 & C & 10 \\ 1940 & C & 10 \\ 1950 & C & 12 \\ 1966 & C & 16 \\ 1952 & C & 10\end{array}$

S
S
S
$G$
$G$

$\begin{array}{ll}41 & w \\ 44 & w \\ 34 & w \\ 56 & w\end{array}$

1953

1959

1952

$1949 \mathrm{C}$

$1940 \mathrm{C}$

$1966 \mathrm{P}$

$1951 \mathrm{C}$

1952

$1952=$
$1952=$
$1966=$

$1966=$
$1966=$

1966

$1970 \mathrm{C}$
1926

WESTMINSTER

$\begin{array}{lll}1961 & - & -- \\ 1964 & B & -- \\ 1964 & W & -- \\ 1939 & C & 6 \\ 1939 & D & 36\end{array}$

$\begin{array}{rr}22 & T \\ 20 & T \\ 150 & \end{array}$

1918 D 42

$28 W 12$

$\begin{array}{lll}= & D & 24 \\ = & D & 24\end{array}$

1949 U 40

$\begin{array}{rlll}65 & w & 18 & --\end{array}$

1948

1941
1948

$\begin{array}{rrr}1948 & D & 24 \\ 1943 & C & 6\end{array}$

$14 W$

19490

$1948 \mathrm{C}$

$1941 \quad c$

$\begin{array}{rrr}1918 & V & 1 \\ 1926 & 0 & 36\end{array}$

$18 w$
* BOYLSTDN WD

DAHM MAYNARD

SAGILAMPI BROS LAUGHLIN SCHOOL LEHTONEN OSCAR WISWELL WALTEK 1919 D 30

$\begin{array}{lllr}14 & w & - & T \\ 14 & w & 95\end{array}$

$16 w \quad--95$

$16 \quad T$

$\begin{array}{rlll}133 & w & -- & - \\ 30 & = & -\end{array}$

6

6
261
22
9$$
\text { - U }
$$

RAHAIM PETER

UPTON SCHOOL

BABINEAU A

CHATA WAINO

BENNETT W C

OLD MILL REST

BAUM RUSSELL
LEMIEUR HENRY

LEMIEUR HENRY
WESTMINSTER

$\begin{array}{rlllllll}1945 & C & 6 & X & 300 & U & 20 & =\end{array}$

$\begin{array}{cccccccc}-1 & C & 6 & X & 190 & 108 & -- \\ 1949 & C & 6 & 0 & 143 & 143 & R \\ -1944 & 0 & 30 & - & 89 & 30 & 30 & -\end{array}$

GARDNER ST HOSP

GAKDNER ST HOSP

GARDNER ST HOSP

GARONER ST HOSP

1944030

$\begin{array}{rrr}-- & D & 36 \\ 1946 & C & 6\end{array}$

$\begin{array}{rrrrrrrr}1948 & v & -- & - & 12 & w & -- & R \\ 1969 & v & 2 & - & 118 & w & 14 & =\end{array}$

GARDNER ST HOSP

GARDNER ST HOSP

WESTMINSTER

WESTMINSTER

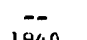

$52 \quad--\quad 95$

95
5
95
5
5

$1940=-2$

-- $-=$

$\begin{array}{lll}22 & 1 & - \\ 24 & 1 & 28\end{array}$

$\begin{array}{llll}20 & 1 & -2 & 5\end{array}$

18

$\begin{array}{rrrrrrrr}4 & 8-72 & P & 1215 & 34 & 48 & D & P \\ 18 & 3-60 & H & 10 & 50 & 24 & - & - \\ 11 & -74 & U & -- & -- & -- & D & - \\ 13 & -74 & U & -- & -- & - & 0 & - \\ 9 & -74 & U & -- & -- & -- & D & - \\ 16 & -74 & U & 32 & -- & -- & D & C\end{array}$

$\begin{array}{rrrrrrrr}-- & -- & P & 150 & 6 & 440 & - & - \\ - & -- & P & 165 & -- & 216 & \bar{D} & - \\ 11 & 6-50 & H & 150 & 7 & - & D & - \\ 5 & 7-66 & P & 251 & 12 & 48 & D & - \\ 6 & 6-52 & P & 300 & 11 & 48 & - & P\end{array}$

$\begin{array}{rrrrrrrr}9 & -53 & U & 725 & 10 & 144 & D & - \\ -- & -- & H & 20 & -- & -- & - & - \\ 30 & 6-52 & H & 2 & -- & -- & - & - \\ 33 & 9-45 & H & 4 & -- & -- & - & - \\ 21 & 12-49 & H & 6 & -- & -- & - & -\end{array}$

$\begin{array}{ccccc}-- & H & -- & =- & =- \\ 12-66 & 1 & 18 & =- & =- \\ 8-51 & H & 15 & =- & =-\end{array}$

$\begin{array}{cccccc}38 & 8-51 & H & .5 & =- & --\end{array}$

$\begin{array}{rrrrrrrr}-- & -- & U & -- & -- & -- & 0 & - \\ 5 & 10-52 & U & 100 & 3 & 6 & 0 & = \\ 22 & 7-66 & U & -- & -- & -- & 0 & - \\ 10 & 7-66 & U & -- & -- & -- & 0 & - \\ 17 & 7-66 & U & 20 & -- & -- & D & - \\ 20 & 5-70 & P & 402 & 24 & 48 & D & P \\ -- & -- & U & -- & -- & -- & - & -\end{array}$

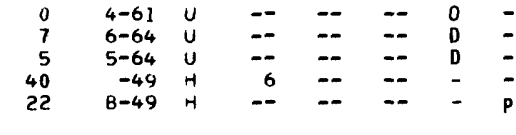

17
$P 12$
$P 13$

$\begin{array}{llllll}8-49 & 5 & -- & -- & = & - \\ 8-49 & 1 & -- & -- & = & -\end{array}$

$\begin{array}{rrrrrr}\text { P13 } & 8-49 & T & -- & =- & = \\ \text { P15 } & 8-49 & T & -- & =- & =- \\ 5 & 8-49 & H & -- & -- & --\end{array}$

25
25
--

-39
-48

-49
-43

$\begin{array}{lll}1 & -- & = \\ 10 & = & = \\ -- & = & =\end{array}$

$\frac{14}{7}$

-49
$8-49$

$8-49$

$\begin{array}{rrr}-- & -- & = \\ 2 & = & = \\ 7 & =- & = \\ -- & = & =\end{array}$

2

$8-49$
$5-51$

$5-51$
$B-49$
$B-49$

30

$\therefore$

10 8-49 5 -.

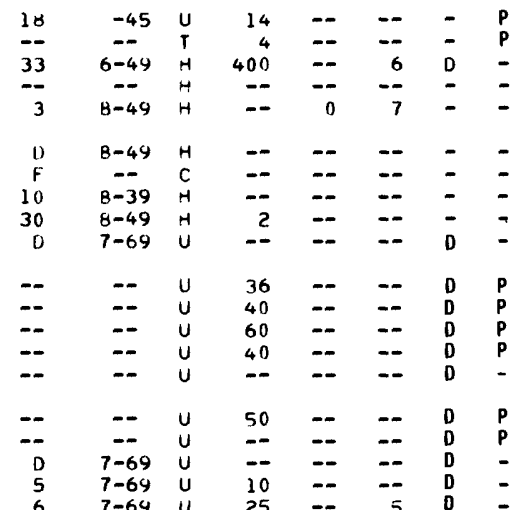


LOCAL

WELL

LATITUDE- ALTILONGITUDE OF LSO

YEAR/ TI- WELL DRILLED ETER IISH । \begin{tabular}{l|l|l} 
(IN) I ISH I (FT)! BEO- & ROCK
\end{tabular}

LEVELINATE IUSE YIELOI DO ITIMF LOG OW WESTMINSTEK - -CONTINUED

$52 \quad 423051 N 0713327.1 \quad 900$ 55 423257N0715533.1 1090 423253N0715544.1 1120 7 423252N0715547.1 1115

$58 \quad 423256 N 0715548.1 \quad 1088$ $59423128 \mathrm{NO} 11530 \mathrm{~B} .1925$ $\begin{array}{lll}60 & 423129 N 0715309.1 & 905 \\ 61 & 423132 N 071530 B_{1} 1 & 900\end{array}$ $\begin{array}{lll}61 & 423132 N 071530 B .1 & 900 \\ 62 & 423432 N 0115417.1 & 835\end{array}$

$63 \quad 423337 N 0715459.1 \quad 1135$ $64 \quad 423339 N 0715459.11120$ $65423353 N 0715509.11050$ $\begin{array}{rrr}66 & 423351 N 0715513.1 & 1060 \\ 67 & 423428 N 0715311.1 & 790\end{array}$

$68 \quad 423435 N 0715314.1 \quad B 00$ 69423450 N0715344.1 805 $70 \quad 423459$ NO $115402.1 \quad 830$ 72 423558N0715438.1 845

73 423551N0715436.1 830 $74 \quad 423516 N 0715418.1 \quad 815$ $\begin{array}{lll}76 & 423131 N 0715320.1 & 900 \\ 77 & 423058 N 0715254.1 & 895\end{array}$ 78 423036N0715231.1 920

$79423106 N 0715241.1920$ $80 \quad 423234 N 0715453.1 \quad 1130$ $\begin{array}{lll}82 & 423511 N 0115219.1 & 1005 \\ 83 & 423311 N 0715640.1 & 1135\end{array}$ $\begin{array}{llr}83 & 423311 N 0715640.1 & 1135 \\ 84 & 423331 N 0715322.1 & 955\end{array}$

85 423249N0715237.1 900 $86 \quad 423149 N 0715421.1 \quad 1098$ $89423049 N 0715407.21152$ 92423256 N0715236.1 885

94 423153N0715224.1 905 $95423041 N 0715237.1902$ $\begin{array}{lll}96 & 423521 N 0715417.1 & 855 \\ 98 & 423137 N 0715219.1 & 955\end{array}$ $99 \quad 423135 N 0715233.1 \quad 889$

$101423528 N 0715403.1980$ $102423538 N 0715401.17010$ $\begin{array}{lll}104 & 423156 N 0715224.1 & 895 \\ 110 & 423151 N 0715258.1 & 889\end{array}$ - 112 423612N071544B.1 B35

* 113423620 NO715208.1 775 $\begin{array}{rrr}114 & 423109 N 0715454.1 & 1238 \\ 115 & 423305 N 0715201.1 & 741\end{array}$ $\begin{array}{rrr}115 & 423305 N 0715201.1 & 741 \\ 116 & 423126 N 0715333.1 & 952\end{array}$ * 117 423221N071ל618.1 1132

w 118 423328N0113313.1 915 * $120423057 N 0715509.11235$ w 123 423309N0715523.1 1092

w 126 423122N0115334.1 955

w $127 \quad 423213 N 0715630.1 \quad 1120$ w 130 423215N0715409.1 1175 * 131 423111N0715241.1 905

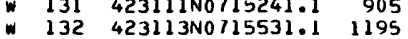

* $134 \quad 423433 \mathrm{~N} 0715314.1 \quad 782$ $\begin{array}{rrr}135 & 423232 N 0715428.1 & 1121 \\ 136 & 423137 N 0115236.1 & 890\end{array}$ * 136 423116N0715452.1 1238 * 138 423304N0715325.1 925

* 141423345 N0115228.1 680 $142 \quad 423116 N 0715118.1$
$-143423307 N 0715211.1$ * $143423307 N 0715211.1 \quad 784$ w 145 423251N0715539.1 1105 $146 \quad 423255 N 0715537.11090$

$12422023 N_{0} 714714.1610$

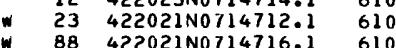

$\begin{array}{lccr}\text { WESTMINSTER } & 1969 & V & 2 \\ \text { SMITH WATER CO } & 1954 & 0 & - \\ \text { WESTMINSTER } & 1969 & V & 2 \\ \text { WESTMINSTER } & 1969 & V & 2 \\ \text { WESTMINSTER } & 1969 & V & 2 \\ & & & \\ \text { WESTMINSTER } & 1969 & V & 2 \\ \text { WESTMINSTER } & 1970 & V & 2 \\ \text { WESTMINSTER } & 1970 & & 2 \\ \text { WESTMINSTER } & 1 N 70 & w & \\ \text { HONKALA LOUIS F } & -- & \mathrm{U} & 30\end{array}$

MICHELSON KEN $1965 P$

$\begin{array}{lclr}\text { WOCSKI HENRY } & 1965 & P & 6 \\ \text { ECKELS GEORGE R } & -- & 0 & 48\end{array}$

WIINIKAINEN W 1948 -

PAGE CHARLES $E$ 1951 C 6

HAY ADAM

PUCKO PAUL

KAMILA DAVID A

WALLIN GEORGE I -. $0-40$

TN GEORGE

STOL Z IRWIN

HARRINGTON F

DIBATTISTA UINO 1925

BRAGOON ROGER N

$\begin{array}{llrr}\text { BRAGOON ROGEK N } & 1951 & 0 & 32 \\ \text { WESTMNSTR AQUED } & 1951 & 0 & 109 \\ \text { HEIKKURINEN J } & 1963 & - & 6\end{array}$

MARCEAU PAUL

LANISA TOIVO

RAMEAU RICHARO

DCONNDR PHILIP

$\begin{array}{llll}\text { ALLEN } & & 1965 & - \\ \text { BEAUREGARD V } & 1964 & P \\ \text { BEAUREGARD V M } & 1949 & \mathrm{C}\end{array}$

BIRCH WARREN

BONINI ANTONID $1963-$

GROWN STEPHAN S 1964 P

CORMIER EMILE F 1971

ENGMAN WALTER

GILLILANO JOHN

GRAVES JDHN

HUGHES RICHARO

ILVONEN TOIVO

$1964 P$

JARVELA EOWAKD

JARVIS STEPHEN

$\begin{array}{llll}\text { JESS CARL } & 1962 & P & 6 \\ \text { KASPER JAMES E } & 1965 & \sim & 6\end{array}$

LALIBERTE MENKY -.. - -

LEBLANC L O SR 1968

MAKELA OLAVI

MALONEY JAMES

MATESOWICZ Z

MUROOCK WARREN

OBRIEN WM

PHELPS RUSSELL

QUINN THOMAS

RAITA AARO

$1965 \mathrm{P}$

$1971 \mathrm{C}$

RINGQUIST CARL

SARGEANT JOHN

SDARI LYLLI

$\begin{array}{lll}\text { SOARI LYLLI } & 1971 & P \\ \text { TORNI WALTER } & 1965 & - \\ \text { TRINQUE LIONEL } & 1964 & P\end{array}$

DECOTONE PROD

LEOM ST FOREST
ADV COATINGS CO

WESTMINSTER

$1970 \mathrm{P}$

$\begin{array}{ll}1966 & P \\ 1966 & P\end{array}$

1966 P

1964

$1971 P$

$\begin{array}{ll}1971 & P \\ 1953 & C\end{array}$

WESTMIÑSTER

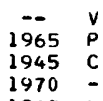

$1970=$

1969 w

\begin{tabular}{l|l}
2 & 5 \\
\hline 2 & - \\
2 & 5 \\
2 & 5 \\
2 & 5 \\
2 & 5 \\
2 & 5 \\
2 & 5 \\
30 & 0
\end{tabular}

$\begin{array}{lll}36 & T & 50 \\ 20 & 1 & -19 \\ 19 & T & 19 \\ 25 & \mathbf{T} & 45 \\ 25 & \mathbf{1} & 45 \\ 34 & 1 & 45 \\ 47 & \mathbf{1} & 58 \\ 73 & \mathbf{8 5} \\ 45 & 0 & 51 \\ 12 & -1 & \end{array}$

$U$
$8 P$
$-R$
$R$
$R$
SR
$2 S$
$4 S$
$S$
$R$

$\begin{array}{rrrrrrrr}7 & 7-69 & U & 60 & -- & -- & D & P \\ -- & -- & P & -- & -- & -- & - & P \\ D & 7-69 & U & -- & -- & -- & D & - \\ 3 & 7-69 & U & 40 & -- & -- & D & P \\ 6 & 7-69 & U & 40 & -- & -- & D & - \\ 0 & 10-69 & U & 45 & -- & -- & D & - \\ 14 & 12-70 & U & 32 & 2 & 2 & D & - \\ 4 & 10-70 & U & 34 & 7 & 336 & D & P \\ 2 & 11-70 & U & 10 & -- & -- & D & P \\ 6 & -71 & H & 3 & -- & -- & - & -\end{array}$

$180 \# 63$

108
12
14

240

70
260
320
160

WORCESTER
MONTROSE DAIRY 1936 MEOLA DAIRY BAR 1942

MEOLA ANTMONY 1942
85

150
80

102

102
17

12

165

108

14
230

35

14
157

157

120

320

405

225
240

126

126
175
155
105

155
105

$260 * 120$

97

505

150
330

220

200
200
495

265

110

185

189
440

440
73
140

140

110
70

160

470
258

160
65

39
275
400

400
350

350
60

\begin{tabular}{r}
24 \\
16 \\
\hdashline- \\
15 \\
-- \\
131 \\
60 \\
6 \\
22 \\
2 \\
20 \\
20 \\
-0 \\
60
\end{tabular}

$\begin{array}{ll}63 & 0 \\ 50 & 0 \\ -- & T \\ 15 & 0 \\ -- & R\end{array}$

$\begin{array}{cccccccc}30 & 8-65 & H & 3 & -- & -- & - & - \\ 30 & -- & H & B & -- & -- & = & - \\ 1 & 10-72 & H & -- & -- & -- & = & - \\ 30 & -70 & H & 14 & -- & -- & - & = \\ -- & -- & H & -- & -- & -- & - & -\end{array}$

$\begin{array}{rrr}15 & -- & 30 \\ 17 & R & 1\end{array}$

- $15 \quad \ldots \quad$ -

$-51$

-51
$9-70$

$9-72$

$9-72$

-69
$9-72$

$10-72$

9-72

$\begin{array}{ll}-- & R \\ -- & U\end{array}$

70

10
3
22
18

22

18
90

15
59
20

17
5
--

23
62

85

19

-.

$\begin{array}{rrr}3 & \cdots & --\end{array}$

97

60

$22 \quad 40$

20
20
--
60
28

28

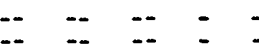

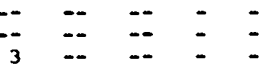

$\begin{array}{lllll}-- & H & 26 & - & -\end{array}$

$7-65$
--

$7 \quad 11-63$

17

$=$

$\begin{array}{ccc}- & - & H \\ -- & - & H \\ 0 & 10-69 & H\end{array}$

$\begin{array}{rrrrrrrr}+3 & 1-65 & H & 24 & -- & 2 & - & - \\ 35 & 11-64 & H & 3 & -- & -- & - & -\end{array}$

$\begin{array}{rrrrrrr}32 & 8-65 & H & 50 & -- & -- & - \\ - & -- & H & \ldots & -. & -- & -\end{array}$

$\begin{array}{rrrrrrr}-- & - & H & 7 & -- & -. & - \\ -2 & 9-65 & H & 12 & -- & -- & -\end{array}$

$\begin{array}{ccccccc}4- & -- & H & 4 & - & -- & - \\ -- & -- & H & -- & -- & - & -\end{array}$

$\begin{array}{ccccccc}-- & - & H & 10 & - & - & - \\ - & -- & H & 12 & - & - & -\end{array}$

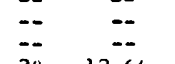

$30 \quad 8-71$

40

$10-65$

18

23
32
18

--
$2-65$
$3-45$
--
--

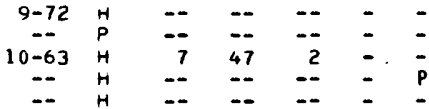


Table 1A. -- Description of selected wells, test wells and borings

[a dash indicates no data are available]

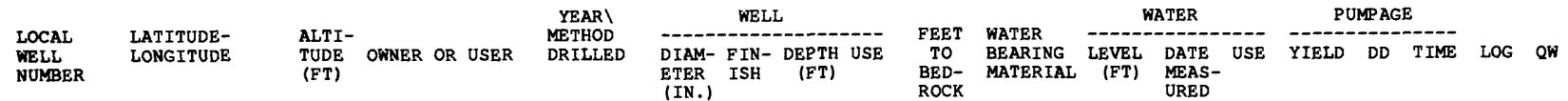

BOLTON

$\begin{array}{llllllll}\text { W } & 133 & 4225230713954.1 & 420 & \text { SCHULTZ JOHN H } & 1981 & \mathrm{P} & 6 \\ \text { W } & 134 & 4227590713741.1 & 275 & \text { DUNELLS GEORGE } & 2-82 & \mathrm{P} & 6 \\ \text { w } & 135 & 4228010713752.1 & 265 & \text { RUSSELL BRUCE } & 1-80 & \mathrm{P} & 6 \\ \text { W } & 136 & 4225490713850.1 & 415 & \text { INT'L GOLF CLUB } & 4-81 & \text { C } & \\ \text { W } & 137 & 422653071375501 & 405 & \text { LEDUC RICHARD } & 8-82 & \mathrm{P} & 6 \\ & & & & & & \\ \text { W } & 138 & 422653071375301 & & \text { LEDUC RICHARD } & 6-82 & \mathrm{P} & 6 \\ \text { W } & 139 & 422813071373701 & 285 & \text { DUNELLS GEORGE } & 9-81 & \mathrm{P} & 6\end{array}$

$\begin{array}{rrrrrrrrrrrrr}\mathrm{X} & 224 & \text { W } & 2 & \mathrm{H} & 15 & 10-81 & \mathrm{H} & 30 & - & - & - & - \\ \mathrm{X} & 400 & \text { W } & 4 & \mathrm{H} & -1 & 05-82 & \mathrm{H} & 3 & - & - & - & - \\ \mathrm{X} & 50 & \text { W } & 16 & \mathrm{H} & & & \mathrm{H} & 100 & - & - & - & - \\ \mathrm{P} & 128 & \text { W } & 128 & \mathrm{G} & 72 & 11-81 & \mathrm{H} & 1170 & 6 & 24 & - & - \\ \mathrm{X} & 363 & \text { W } & 108 & \mathrm{H} & 50 & 09-82 & \mathrm{H} & 8 & - & - & - & - \\ \mathrm{X} & 303 & \text { W } & 98 & \mathrm{H} & 50 & 08-82 & \mathrm{H} & 5 & - & - & - & - \\ \mathrm{X} & 343 & \text { W } & 31 & \mathrm{H} & 20 & 10-81 & \mathrm{H} & 12 & - & - & - & -\end{array}$

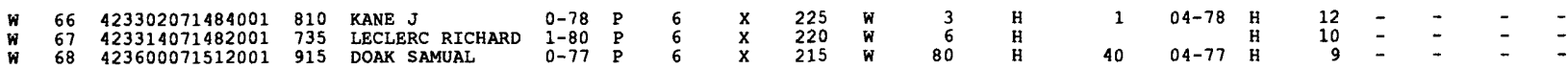

FITCHBURG

GROTON

\begin{tabular}{|c|c|c|c|c|}
\hline $\begin{array}{l}1 \\
87 \\
88 \\
89 \\
90\end{array}$ & $\begin{array}{l}423806071391601 \\
423908071342801 \\
423907071342301 \\
423906071342701 \\
423831071342401\end{array}$ & $\begin{array}{l}275 \\
245 \\
240 \\
250 \\
230\end{array}$ & $\begin{array}{l}\text { GROTON } \\
\text { MORGAN } \\
\text { MORGAN } \\
\text { MORGAN } \\
\text { MORGAN }\end{array}$ & $\begin{array}{l}\text { TOWN } \\
\mathrm{C} \\
\mathrm{C} \\
\mathrm{C} \\
\mathrm{C}\end{array}$ \\
\hline $\begin{array}{l}91 \\
92 \\
93 \\
94 \\
95\end{array}$ & $\begin{array}{l}423906071342702 \\
423910071342501 \\
423901071342901 \\
423831071341401 \\
423910071341401\end{array}$ & $\begin{array}{l}240 \\
260 \\
220 \\
235 \\
240\end{array}$ & $\begin{array}{l}\text { MORGAN } \\
\text { MORGAN } \\
\text { MORGAN } \\
\text { MORGAN } \\
\text { MORGAN }\end{array}$ & 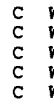 \\
\hline $\begin{array}{r}96 \\
97 \\
98 \\
99 \\
100\end{array}$ & $\begin{array}{l}423910071342801 \\
423904071342401 \\
423901071340201 \\
423822071341601 \\
423821071341601\end{array}$ & $\begin{array}{l}260 \\
230 \\
230 \\
250 \\
250\end{array}$ & $\begin{array}{l}\text { MORGAN } \\
\text { MORGAN } \\
\text { MORGAN } \\
\text { MORGAN } \\
\text { MORGAN }\end{array}$ & $\begin{array}{l}\mathrm{C} \\
\mathrm{C} \\
\mathrm{C} \\
\mathrm{C} \\
\mathrm{C}\end{array}$ \\
\hline $\begin{array}{l}101 \\
102 \\
103\end{array}$ & $\begin{array}{l}423754071325801 \\
423822071342001 \\
423908071340201\end{array}$ & $\begin{array}{l}310 \\
250 \\
230\end{array}$ & $\begin{array}{l}\text { KETCHAM } \\
\text { HILL } \\
\text { MORGAN }\end{array}$ & C \\
\hline
\end{tabular}

$\begin{array}{rllllll}7-84 & \mathrm{~B} & 8 & - & - & \mathrm{U} & \\ 0-80 & \mathrm{P} & 6 & \mathrm{X} & 400 & \mathrm{~W} & 25 \\ 2-80 & \mathrm{P} & 6 & \mathrm{X} & 460 & \mathrm{~W} & 30 \\ 1-80 & \mathrm{P} & 6 & \mathrm{X} & 320 & \mathrm{~W} & 20 \\ 1-80 & \mathrm{P} & 6 & \mathrm{X} & 560 & \mathrm{~W} & 24 \\ 5-81 & \mathrm{P} & 6 & \mathrm{X} & 422 & \mathrm{~W} & 12 \\ 3-80 & \mathrm{~A} & 6 & \mathrm{X} & 380 & \mathrm{~W} & 30 \\ 6-81 & \mathrm{P} & 6 & \mathrm{X} & 582 & \mathrm{~W} & 12 \\ 1-78 & \mathrm{~A} & 6 & \mathrm{X} & 250 & \mathrm{~W} & 38 \\ 7-81 & \mathrm{P} & 6 & \mathrm{X} & 340 & \mathrm{~W} & 27 \\ 3-81 & \mathrm{P} & 6 & \mathrm{X} & 400 & \mathrm{~W} & 27 \\ 9-80 & \mathrm{P} & 6 & \mathrm{X} & 460 & \mathrm{~W} & 7 \\ 0-82 & \mathrm{P} & 6 & \mathrm{X} & 303 & \mathrm{~W} & 9 \\ 1-79 & \mathrm{P} & 6 & \mathrm{X} & 345 & \mathrm{~W} & 77 \\ 1-79 & \mathrm{P} & 6 & \mathrm{X} & 405 & \mathrm{~W} & 82 \\ & & & & & & \\ 1-83 & \mathrm{P} & 6 & \mathrm{X} & 460 & \mathrm{~W} & 20 \\ 7-79 & \mathrm{~A} & 6 & \mathrm{X} & 500 & \mathrm{~W} & 25 \\ 4-81 & \mathrm{P} & 6 & \mathrm{X} & 400 & \mathrm{~W} & 6\end{array}$

$\begin{array}{rrrrrrrr}22 & 08-84 & & - & - & - & - & - \\ 25 & 06-80 & \text { H } & 2 & - & - & - & - \\ 40 & 03-80 & \text { H } & 20 & - & - & - & - \\ & & \text { H } & 15 & - & - & - & - \\ & & \text { H } & - & - & - & - & - \\ 10 & 08-81 & \text { H } & 2 & - & - & - & - \\ 40 & 03-80 & \text { H } & 25 & - & - & - & - \\ 30 & 08-81 & \text { H } & 6 & - & - & - & - \\ 40 & 03-81 & \text { H } & 25 & - & - & - & - \\ 20 & 03-81 & \text { H } & 4 & - & - & - & - \\ 15 & 06-80 & \text { H } & - & - & - & - & - \\ 20 & 09-82 & \text { H } & 9 & - & - & - & - \\ & & \text { H } & 2 & - & - & - & - \\ & & \text { H } & 1 & - & - & - & - \\ 30 & 02-79 & \text { H } & 3 & - & - & - & - \\ 5 & 05-81 & \text { H } & 1 & - & - & - & -\end{array}$

พ $106 \quad 423029071343601 \quad 280$ NEWSHAM W

107422854071350401565 DIRUSSO DONALD

$\begin{array}{llll}108 & 422956071342101 & 520 & \text { NESBEDA PETER }\end{array}$

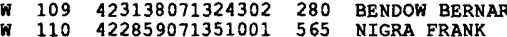

W $111423104071324801 \quad 570$ DECK HOUSE

W $112423137071325501 \quad 300$ GOLD STEPHEN

W 113423107071324601550 ROSATO DAVID

$\begin{array}{lllll}\text { W } & 114 & 422847071343001 & 385 & \text { SHIRING JAMES } \\ \text { W } & 115 & 423049071345702 & 280 & \text { JOHNSON JOHN }\end{array}$

W $116 \quad 423141071342701 \quad 290$ HIGGINS JEFF

$\begin{array}{lllll}W & 117 & 423156071341201 & 345 & \text { NELSON JAME } \\ \text { W } & 118 & 423006071355501 & 370 & \text { DORWARD MR }\end{array}$

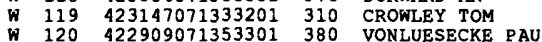

W 121423034071344901280 MARTINEAU DENNI

* 122423223071335501305 NORTZ RICHARD

W 123422843071344601 410 BROGAN EUGENE

$\begin{array}{lllll}\text { w } & 124 & 422903071353601 & 390 & \text { QUINE WILLARD } \\ \text { w } & 125 & 423104071315401 & 260 & \text { FINKEL STEVEN }\end{array}$

W $126 \quad 423244071340501290$ NELSON HAROLD

W $127 \quad 422818071343301 \quad 450$ GIBSON GORDON

W $128 \quad 423106071330501 \quad 550$ HOLMES STEVEN

$\begin{array}{lllll}\text { W } & 129 & 423138071324301 & 280 & \text { BENDOW BERNARD } \\ \text { W } & 130 & 422711071333601 & 280 & \text { HUGHES } J \\ \end{array}$

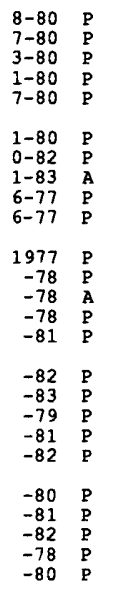

HARVARD

W $138 \quad 423455071422001 \quad 375$ CLARK HOWARD

$\begin{array}{lllll}139 & 423641071461801 & 475 & \text { WOOD JOHN C }\end{array}$

$\begin{array}{lllll}\text { w } & 141 & 423653071420001 & 375 & \text { PASSIOS DAVID } \\ \text { W } & 145 & 423404071430901 & 370 & \text { DOUGLAS DONALD } \\ \text { W } & 146 & 423332071421701 & 365 & \text { GEORGE }\end{array}$

W 147423327071413301300 PATRY RICHARD

W $148 \quad 423251071411101 \quad 305$ NORMANDIN ROBT

W $150 \quad 423400071430001380$ WHITNEY PETER

W $\begin{array}{lllll}152 & 423451071412401 & 385 & \text { FOREST NORMAN } \\ \text { W } & 153 & 423343071415301 & 350 & \text { KULJU } c\end{array}$

\% $156 \quad 423705071420501 \quad 395$ SEAVER ARTHUR

$\begin{array}{lllll}\text { W } & 157 & 423657071454001 & 605 & \text { LOLLI RALPH } \\ \text { W } & 158 & 423349071424201 & 485 & \text { STILLMAN GLEN }\end{array}$

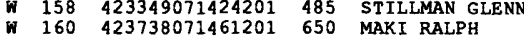

$\begin{array}{llll}161 & 423430071413801 & 370 & \text { MELTULA MARTTI }\end{array}$

$\begin{array}{rlllllrl}-81 & \mathrm{P} & 6 & \mathrm{X} & 300 & \text { W } & 42 & \mathrm{~F} \\ -81 & \mathrm{P} & 6 & \mathrm{X} & 75 & \text { W } & 15 & \mathrm{H} \\ -77 & \mathrm{P} & 6 & \mathrm{X} & 275 & \text { W } & 20 & \mathrm{H} \\ -76 & \mathrm{P} & 6 & \mathrm{X} & 190 & \text { W } & 28 & \mathrm{H} \\ -76 & \mathrm{P} & 6 & \mathrm{X} & 250 & \text { W } & 23 & \mathrm{H} \\ -76 & \mathrm{P} & 6 & \mathrm{X} & 365 & \text { W } & 35 & \mathrm{H} \\ -77 & \mathrm{P} & 6 & \mathrm{X} & 340 & \text { W } & 100 & \mathrm{H} \\ -77 & \mathrm{~A} & 6 & \mathrm{X} & 245 & \text { W } & 50 & \mathrm{H} \\ -80 & \mathrm{~A} & 6 & \mathrm{X} & 140 & \text { W } & 20 & \mathrm{H} \\ -81 & \mathrm{P} & 6 & \mathrm{X} & 375 & \text { W } & 80 & \mathrm{H} \\ -83 & \mathrm{P} & 6 & \mathrm{X} & 325 & \text { W } & 8 & \mathrm{~F} \\ -78 & \mathrm{P} & 6 & \mathrm{X} & 440 & \text { W } & 14 & \mathrm{H} \\ -79 & \mathrm{P} & 6 & \mathrm{X} & 645 & \text { W } & 165 & \mathrm{H} \\ -78 & \mathrm{P} & 6 & \mathrm{X} & 200 & \text { W } & 2 & \mathrm{H} \\ -79 & \mathrm{P} & 6 & - & 180 & \text { W } & 59 & \mathrm{H}\end{array}$

\begin{tabular}{|c|c|c|c|c|}
\hline$x$ & 100 & ต & 57 & $\mathrm{H}$ \\
\hline$x$ & 240 & ต & 24 & H \\
\hline$x$ & 140 & ต & 13 & H \\
\hline$x$ & 180 & w & 38 & H \\
\hline$x$ & 480 & พ & 5 & H \\
\hline$x$ & 300 & พ & 20 & H \\
\hline$x$ & 125 & w & 8 & $\mathrm{H}$ \\
\hline$x$ & 300 & w & 40 & H \\
\hline$x$ & 260 & w & 19 & H \\
\hline$x$ & 200 & w & 80 & H \\
\hline$x$ & 365 & พ & 70 & H \\
\hline$x$ & 365 & พ & 118 & H \\
\hline$x$ & 180 & พ & 38 & H \\
\hline$x$ & 60 & พ & 4 & H \\
\hline$x$ & 300 & w & 5 & H \\
\hline$x$ & 225 & w & 40 & H \\
\hline$x$ & 290 & $w$ & 135 & $\mathrm{H}$ \\
\hline$x$ & 180 & พ & 28 & H \\
\hline$x$ & 302 & $w$ & 13 & $\mathrm{H}$ \\
\hline$x$ & 355 & $w$ & 20 & H \\
\hline$\lambda$ & 500 & พ & & H \\
\hline$x$ & 215 & พ & 3 & 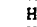 \\
\hline$x$ & 160 & พ & 35 & H \\
\hline$x$ & 200 & $\omega$ & 6 & $\mathrm{H}$ \\
\hline$x$ & 265 & พ & 62 & $\mathrm{H}$ \\
\hline
\end{tabular}

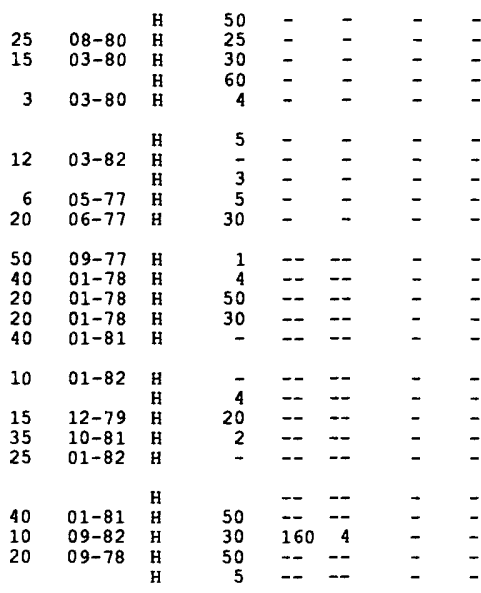

\section{LUNENBURG}


Table 1A. -- Description of selected wells, test wells and borings -(Continued)

[a dash indicates no data are available]

\begin{tabular}{|c|c|c|c|c|c|c|c|c|c|c|c|c|c|c|c|c|c|c|}
\hline \multirow[b]{2}{*}{$\begin{array}{l}\text { LOCAL } \\
\text { WELL } \\
\text { NUMBER }\end{array}$} & \multirow[b]{2}{*}{$\begin{array}{l}\text { LATITUDE- } \\
\text { LONGITUDE }\end{array}$} & \multirow[b]{2}{*}{$\begin{array}{l}\text { ALTI- } \\
\text { TUDE } \\
\text { (FT) }\end{array}$} & \multirow[b]{2}{*}{ OWNER } & \multirow[b]{2}{*}{ OR USER } & \multirow{2}{*}{$\begin{array}{l}\text { YEARI } \\
\text { METHOD } \\
\text { DRILLED }\end{array}$} & \multicolumn{3}{|c|}{ WELL } & \multirow[b]{2}{*}{$\begin{array}{l}\text { FEET } \\
\text { TO } \\
\text { BED- } \\
\text { ROCK }\end{array}$} & \multirow[b]{2}{*}{$\begin{array}{l}\text { WATER } \\
\text { BEARING } \\
\text { MATERIAL }\end{array}$} & \multicolumn{3}{|c|}{ WATER } & \multicolumn{3}{|c|}{$\begin{array}{l}\text { PUMPAGE } \\
\end{array}$} & \multirow[b]{2}{*}{ L.OG } & \multirow[b]{2}{*}{ QW } \\
\hline & & & & & & $\begin{array}{l}\text { DIAM- } \\
\text { ETER } \\
\text { (IN.) }\end{array}$ & $\begin{array}{l}\text { FIN- } \\
\text { ISH }\end{array}$ & $\begin{array}{l}\text { DEPTH USE } \\
\text { (FT) }\end{array}$ & & & (ET) & $\begin{array}{l}\text { DATE } \\
\text { MEAS- } \\
\text { URED }\end{array}$ & USE & YIELD & DD & TIME & & \\
\hline
\end{tabular}

LUNEMBURG - Continued

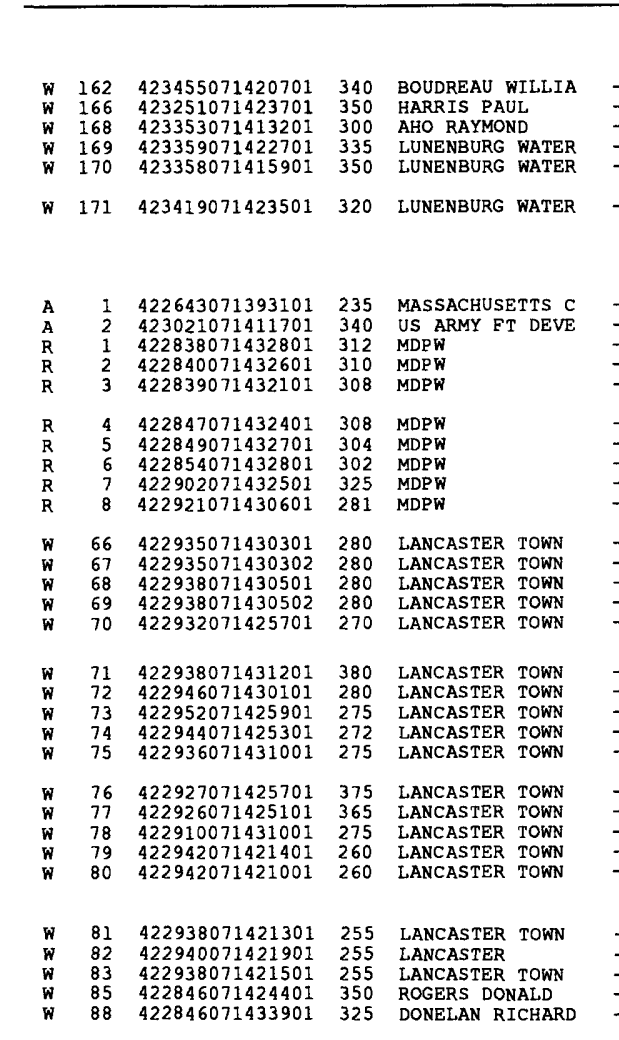

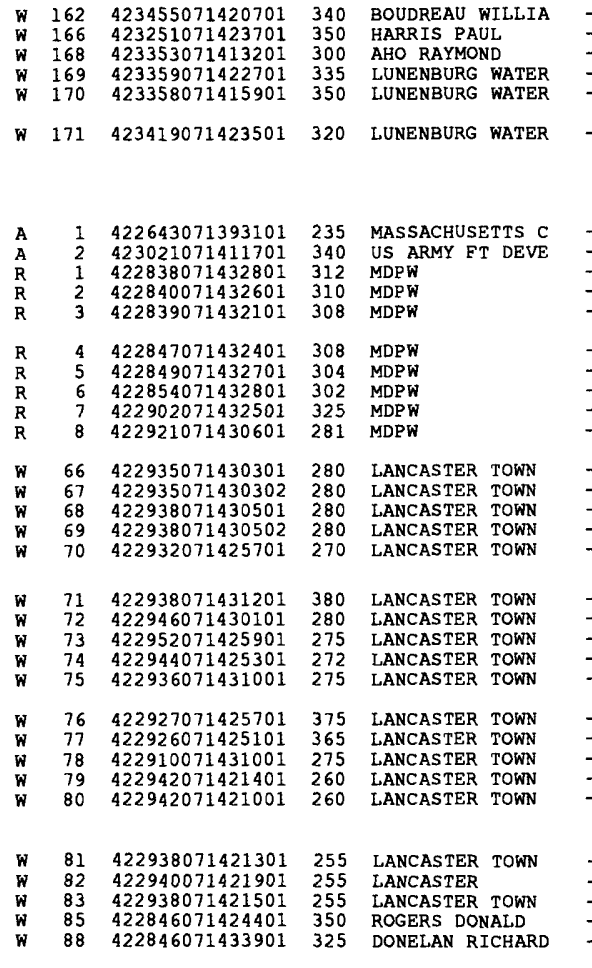

\begin{tabular}{rrr|lr|rrr}
-78 & A & 6 & X & 220 & W & 22 & H \\
-80 & P & 6 & X & 305 & W & 89 & H \\
-78 & P & 6 & - & - & W & 20 & H \\
-77 & C & 48 & P & 46 & W & & R \\
-75 & C & 8 & P & 40 & T & & C \\
-84 & B & 8 & P & 34 & W & & R \\
& & & & & & \\
LANCASTER & & &
\end{tabular}

$\begin{array}{lllll}\mathrm{R} & 1 & 422928071433401 & 381 & \text { MDPW }\end{array}$

R $\quad 422950071434901387$ MDPW

$\begin{array}{rlll}5 \quad 422949071432401 & 319 & \text { MDPW }\end{array}$

$\begin{array}{lll}167 & 423104071460601 & 435 \\ \text { PRIDDY SKIP }\end{array}$

W $168 \quad 423015071485601 \quad 830$ VAILLETTE MICHA W $169423145071464401 \quad 510$ MARSH ROL.AND W $170 \quad 423027071485001 \quad 850$ TREOTIN RICHARD W $173 \quad 423013071463501$ 600 GUILMETTE JAMES

W $174 \quad 422945071463801625$ DIVOLL EARL C
W 52424207071375901222 PEPPERELL TOWN $\begin{array}{llll}52 & 424207071375901 & 222 & \text { PEPPERELL TOWN } \\ 52 & 424207071375902 & 223 & \text { PEPPERELL TOWN }\end{array}$ $\begin{array}{llll}52 & 424207071375903 & 222 & \text { PEPPERELL TOWN }\end{array}$ $\begin{array}{llll}53 & 424212071375901 & 220 & \text { PEPPERELL TOWN } \\ 53 & 424212071375902 & 220 & \text { PEPPERELL TOWN }\end{array}$

W $53 \quad 424212071375903 \quad 221$ PEPPERELL TOWN 54424204071380001226 PEPPERELL TOWN $\begin{array}{llll}54 & 424204071380002 & 226 & \text { PEPPERELL TOWN } \\ 54 & 424204071380003 & 226 & \text { PEPPERELL TOWN }\end{array}$ $\begin{array}{llll}54 & 424204071380003 & 226 & \text { PEPPERELL, TOWN } \\ 55 & 424208071380001 & 224 & \text { PEPPERELL, TOWN }\end{array}$

W $55 \quad 424208071380002 \quad 222$ PEPPERELL, TOWN $\begin{array}{llll}56 & 424208071375901 & 222 & \text { PEPPERELL TOWN }\end{array}$ 59
524207071375802

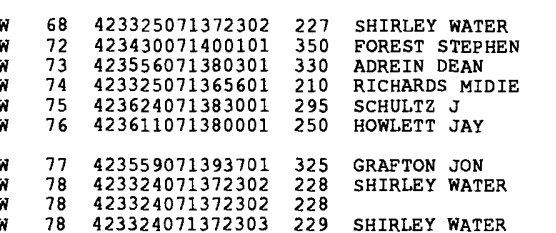

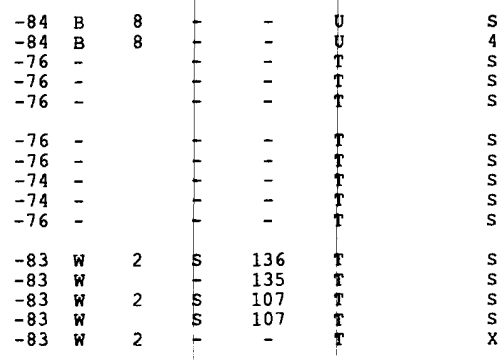

$\begin{array}{llllll}-83 & \text { W } & 2 & X & - & t \\ -83 & \text { A } & 2 & - & - & T \\ -83 & \text { W } & 2 & = & - & T \\ -83 & \text { W } & 2 & = & - & T \\ -83 & \text { W } & 2 & - & -\end{array}$

$\begin{array}{llllll}-83 & W & 2 & \text { S } & 96 & I \\ -83 & W & 2 & - & - & I \\ -83 & W & 2 & 5 & 91 & T \\ -83 & - & 2 & - & - & T \\ -83 & \text { W } & 2 & - & -\end{array}$

$\begin{array}{ll}-83 & W \\ -83 & W \\ -83 & W \\ -77 & P \\ -84 & B\end{array}$ $\begin{array}{rrrrrrrr} & & \text { H } & 4 & -- & -- & - & - \\ 50 & 01-78 & \text { H } & 8 & - & -- & - & - \\ 16 & 10-77 & \text { P } & 225 & - & - & - & - \\ 5 & 06-75 & \text { U } & 140 & 3 & -- & \text { D } & - \\ 2 & 02-84 & \text { H } & - & -- & -- & \text { D } & -\end{array}$

$33108-$

$08-84$

$08-84$

$10-76$
$10-76$
$10-76$

$4 \begin{array}{r}10-76 \\ 10-76\end{array}$

$\begin{array}{rll}4 & 10-76 & U \\ 2 & 05-74 & U \\ 44 & 05-74 & U \\ 6 & 01-76 & U\end{array}$

$-3 \quad 04-83 \mathrm{U}$

$\begin{array}{r}04-83 \\ -\quad 09-83 \\ -\quad 04-83 \\ \hline\end{array}$

-2 09-83

8

5
2
2

04-83-

$04-83=$
$04-83$

$\begin{array}{lll}- & -- & - \\ - & - & -\end{array}$

-2 05-83

\begin{tabular}{l}
$05-83$ \\
$3 \quad 05-83$ \\
\hline
\end{tabular}

U

$\begin{array}{ccc}5 & -- & - \\ 15 & - & -\end{array}$

$\begin{array}{lll}5 & -Z & - \\ - & -- & -\end{array}$

$11 \quad 08-84$

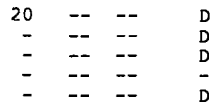

LEOMINSTER

$\begin{array}{rrr}41 & 01-76 & \mathrm{U} \\ & & \mathrm{U} \\ 18 & 01-76 & \mathrm{U} \\ 30 & 08-82 & \mathrm{H} \\ & \\ 6 & 12-75 & \mathrm{H} \\ 30 & 03-77 & \mathrm{H} \\ 30 & 08-77 & \mathrm{H} \\ 8 & 08-80 & \mathrm{H} \\ 30 & 11-78 & \mathrm{H}\end{array}$

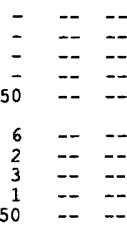

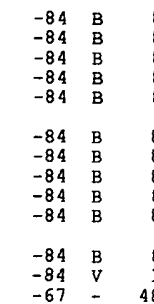

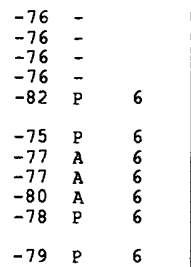

PEPPERELL

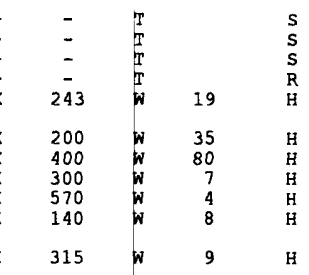

-76
-81
-79 $\begin{array}{lll}-82 & \mathrm{P} \\ -82 & \mathrm{~A}\end{array}$

-81
-84
-84
-84

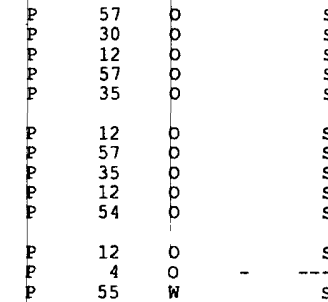

SHIRLEY

53
146
182
300
303
230
405
45
45
21

$10-84$
$10-84$ $10-84 \quad \mathrm{U}$ $12-84$
$12-84$

$12-84 \quad U$

$10-84 \quad U$ $10-84$
$10-84$ $10-84$
$10-84$

$5 \quad 10-84$ 11-83

$$
\begin{aligned}
& \begin{array}{ll}
-- & - \\
-- & -
\end{array} \\
& \because= \\
& --- \\
& =
\end{aligned}
$$$$
\begin{array}{lll}
\overline{7} & -- & - \\
780 & 27 & 120
\end{array}
$$ 
Table 1A. -- Description of selected wells, test wells and borings - (Continued)

[a dash indicates no data are available]

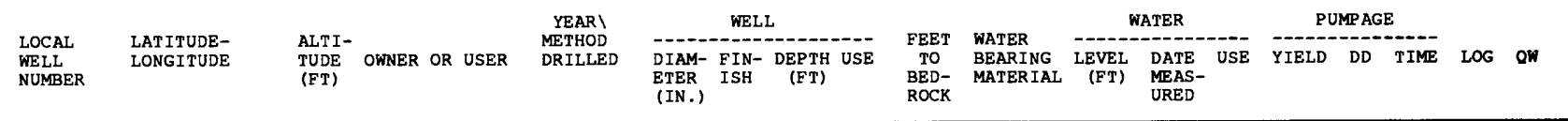

SHIRLEY - Cont inued

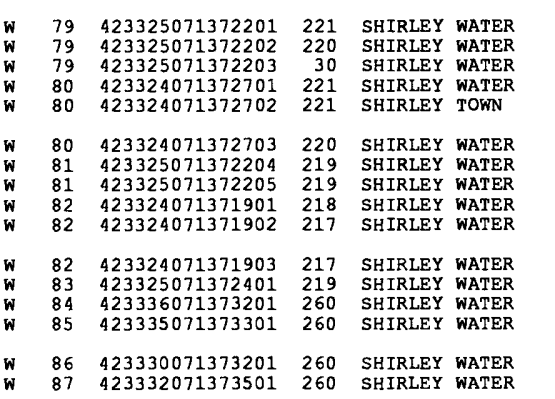

$R$
$R$
$R$
$R$
$R$
$R$
$R$
$R$
$R$
$R$
$R$

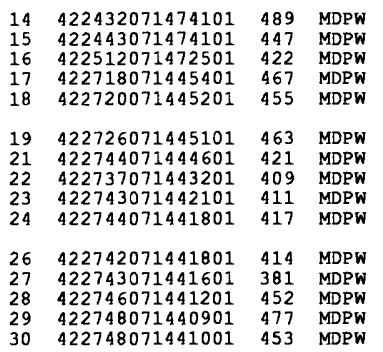$$
\text { tinting }
$$

$31422748071441101 \quad 451 \quad$ MDPW

$\begin{array}{llll}31 & 422748071441101 & 451 & \\ 32 & 422754071435001 & 415 & \text { MDPW }\end{array}$

$\begin{array}{llll}33 & 422754071435001 & 415 & \text { MDPW } \\ 34 & 422804071435101 & 360 & \text { MDPW }\end{array}$

$\begin{array}{llll}35 & 422816071433901 & 342 & \text { MDPW }\end{array}$

$36 \quad 422819071433401 \quad 332$ MDPW

$\begin{array}{lllll}R & 38 & 422822071433301 & 324 & \text { MDPW } \\ R & 39 & 422824071433301 & 323 & \text { MDPW }\end{array}$

$\begin{array}{lllll}R & 39 & 422824071433301 & 323 & \text { MDPW } \\ R & 40 & 422827071433001 & 317 & \text { MDPW }\end{array}$

W $110 \quad 422631071484602 \quad 450$ STERLING TOWN W $125 \quad 422429071441501 \quad 460$ MUSSELLO RICHAR $\begin{array}{lllll}W & 126 & 422456071450201 & 450 & \text { O'TOOLE R RCHARD } \\ \text { W } & 127 & 422632071470301 & 725 & \text { MEOLA ANTHONY }\end{array}$

w $128 \quad 422409071472301$ 430 LUKSHA JOESEPH

W $129422806071440301 \quad 400$ FORD JOHN M M

W 132 422817071450601 480 PRESCOTT MANSON

$\begin{array}{lllll}\text { W } & 133 & 422527071480201 & 420 & \text { JOSTI LAWRENCE } \\ \text { W } & 134 & 422526071480501 & 420 & \text { LAPRADE DAVID }\end{array}$

W $135 \quad 422632071452801 \quad 610$ HOGLUND CHARLES

$\begin{array}{lllll}\text { W } & 136 & 422835071465501 & 590 & \text { CAISSE BARRY L } \\ \text { W } & 137 & 422658071451301 & 475 & \text { LANCASTER STERL }\end{array}$

$\begin{array}{lllll}W & 137 & 422658071451301 & 475 & \text { LANCASTER STERI } \\ W & 138 & 422635071465801 & 740 & \text { PICHIERRI RON }\end{array}$

W 139422424071461801 440 BLANCHFLOWER RI

W $140 \quad 422436071444201 \quad 450$ BAUMAN R

W $141 \quad 422413071444301 \quad 500$ SMITH TED H

$\begin{array}{lllll}\text { W } & 141 & 422413071444301 & 500 & \text { SMITH TED } \\ \text { W } & 141 & 422822071440101 & 350 & \text { STERLING TOWN }\end{array}$

$\begin{array}{lllll}\mathbf{W} & 141 & 422822071440101 & 350 & \text { STERLING TOWN } \\ \mathbf{W} & 143 & 422827071434902 & 330 & \text { STERLING TOWN } \\ \mathbf{W} & 144 & 422827071434901 & 330 & \text { STERLING TOWN }\end{array}$

W $145 \quad 422828071435601 \quad 350$ STERLING TOWN

W $146 \quad 422756071442901 \quad 385$ STERLING TOWN

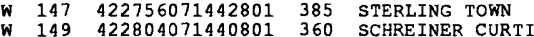

$\begin{array}{lllll}W & 149 & 422804071440801 & 360 & \text { SCHREINER CURT } \\ W & 150 & 422750071442501 & 380 & \text { HAYES DONALD }\end{array}$

W $151422521071480301 \quad 420$ CUTLER ROBERT $w$ W $152 \quad 422815071435201 \quad 345$ STERLING TOWN $\begin{array}{lllll}W & 153 & 422812071440001 & 350 & \text { STERLING TOWN } \\ W & 154 & 422810071440501 & 360 & \text { STERLING TOWN }\end{array}$ W $155422448071472401 \quad 420$ STERLING TOWN

W $156 \quad 422447071472101 \quad 440$ STERLING TOWN

$\begin{array}{lllllll}01-85 & U & - & -- & -- & D & - \\ 01-85 & U & - & -- & -- & - & = \\ 01-85 & U & - & -- & - & - & = \\ 01-85 & U & - & -- & -- & D & - \\ 01-85 & U & - & -- & -- & - & - \\ 01-85 & U & - & -- & -- & - & - \\ 12-84 & U & - & -- & -- & - & - \\ 12-84 & U & - & -- & -- & - & - \\ 11-84 & U & - & -- & -- & D & - \\ 11-84 & U & - & -- & - & - & - \\ 11-84 & U & - & -- & -- & - & - \\ 08-82 & U & - & -- & -- & - & - \\ 08-82 & U & - & -- & -- & D & - \\ 08-82 & U & - & -- & -- & D & - \\ 08-82 & U & - & -- & -- & D & -\end{array}$

STERLING

$\begin{array}{rlllll}-76- & - & - & T & & 75 \\ -76- & - & - & T & \\ -76- & - & - & T & 3 \\ -76- & - & - & T & 3 \\ -76- & - & - & T & \\ -76- & - & - & T & \\ -76- & - & - & T & \\ -76- & - & - & T & \\ -76- & - & - & T & \\ -76- & - & - & T & \\ -76- & - & - & T & \\ -76- & - & - & T & \\ -76- & - & - & T & 10 \\ -76- & - & - & T & 3 \\ -76- & - & - & T & 39\end{array}$

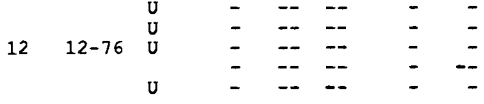

$$
\begin{aligned}
& \begin{array}{rlllllll}
1 & 11-76 & U & - & -- & - & - & - \\
39 & 11-76 & U & - & -- & -- & - & \overline{-} \\
42 & 11-76 & U & - & -- & - & - & =
\end{array} \\
& \begin{array}{llllllll}
40 & 11-76 & U & - & -- & - & - & - \\
16 & 11-76 & U & - & -- & -- & - & - \\
& & U & - & -- & -- & - & \overline{-} \\
& & U & - & - & - & - & -
\end{array}
\end{aligned}
$$

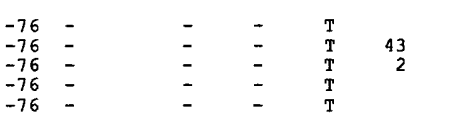$$
\begin{array}{llll}
-76- & - & - & T \\
-76 & - & - & T \\
-76 & - & - & T \\
-76 & - & - & T \\
-76- & - & - & T
\end{array}
$$$$
\begin{array}{rrrrrrrr}
-73 & \mathrm{C} & 30 & \mathrm{P} & 76 & \text { W } & & \mathrm{G} \\
-77 & \mathrm{P} & 6 & \mathrm{X} & 525 & \mathrm{~W} & 5 & \mathrm{H} \\
-79 & \mathrm{P} & 6 & \mathrm{X} & 305 & \mathrm{~W} & 51 & \mathrm{H} \\
-82 & \mathrm{~A} & 6 & \mathrm{X} & 460 & \mathrm{~W} & 50 & \mathrm{H} \\
-79 & \mathrm{P} & 6 & \mathrm{X} & 575 & \mathrm{~W} & 12 & \mathrm{H}
\end{array}
$$$$
\begin{array}{rrrrrrrr}
-78 & \text { A } & 6 & \text { X } & 300 & \text { W } & 109 & \text { H } \\
-79 & \text { A } & 6 & \text { X } & 500 & \text { W } & 42 & \text { H } \\
-78 & \mathrm{P} & 6 & \text { X } & 200 & \text { W } & 19 & \text { H } \\
-78 & \text { P } & 6 & \text { P } & 102 & \text { W } & 102 & \text { S } \\
-77 & \mathrm{P} & 6 & \text { X } & 205 & \text { W } & 118 & \text { H }
\end{array}
$$

$-77 \mathrm{P}$

$\begin{array}{ll}-76 & P \\ -78 & P \\ -77 & P\end{array}$ $\begin{array}{rrrr}185 & \text { W } & 8 & \text { H } \\ 275 & \text { W } & 38 & \text { H } \\ 185 & \text { W } & 35 & \text { H } \\ 140 & \text { W } & 32 & \text { H } \\ 200 & \text { W } & 19 & \text { H }\end{array}$

$\begin{array}{ll}-78 & P \\ -80 & P \\ -82 & \text { W }\end{array}$

$\begin{array}{rrr}600 & \text { W } & 45 \\ 440 & \text { W } & 96\end{array}$

$\begin{array}{lllll}-82 & \mathrm{~W} & 3 & \mathrm{P} & 70\end{array}$

$\begin{array}{ll}-82 & W \\ -82 & P \\ -82 & P \\ -84 & B \\ -84 & B\end{array}$

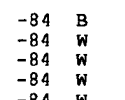

$\begin{array}{ll}-84 & W \\ -84 & W\end{array}$

40
40


1
3
3

5
40
54
40
10
20
45
10

60
15
30
30
15

15
2
2

$\begin{array}{ll}40 & 11-76 \\ 40 & 11-76\end{array}$

$$
\begin{array}{ll}
0 \\
\mathrm{U}
\end{array}
$$$$
\bar{\vdots}
$$$$
\begin{array}{cccc}
--- & - & - & - \\
-- & - & - & -
\end{array}
$$

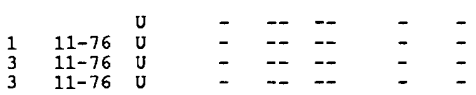
$11-76$ v

$06-73$

$4 \quad 07-82 \quad \overline{\mathrm{H}}$

$01-78$
$09-79$

$09-78$

$04-78$ H

$12-77$
$04-76$

$04-76$

$\begin{array}{ll}01-78 & \mathrm{H} \\ 04-77 & \mathrm{H}\end{array}$

$07-77$

$\begin{array}{ll}10-80 & \mathrm{H} \\ 09-82 & \mathrm{U}\end{array}$

$09-82$ U

$09-82$ U

$\begin{array}{ll}56 & T \\ - & T \\ - & T \\ 13 & 0 \\ 12 & 0\end{array}$

(1)

$09-82$
$08-84$
$08-84$

10-84

$\begin{array}{ll}09-84 & U \\ 09-84 & U\end{array}$

$\begin{array}{ll}09-84 & U \\ 09-84 & U\end{array}$

$\begin{array}{ll}09-84 & U \\ 09-84 & U\end{array}$

09-84 U 
Table 1A. -- Description of selected wells, test wells and borings-(Continued)

[a dash indicates no data are available]

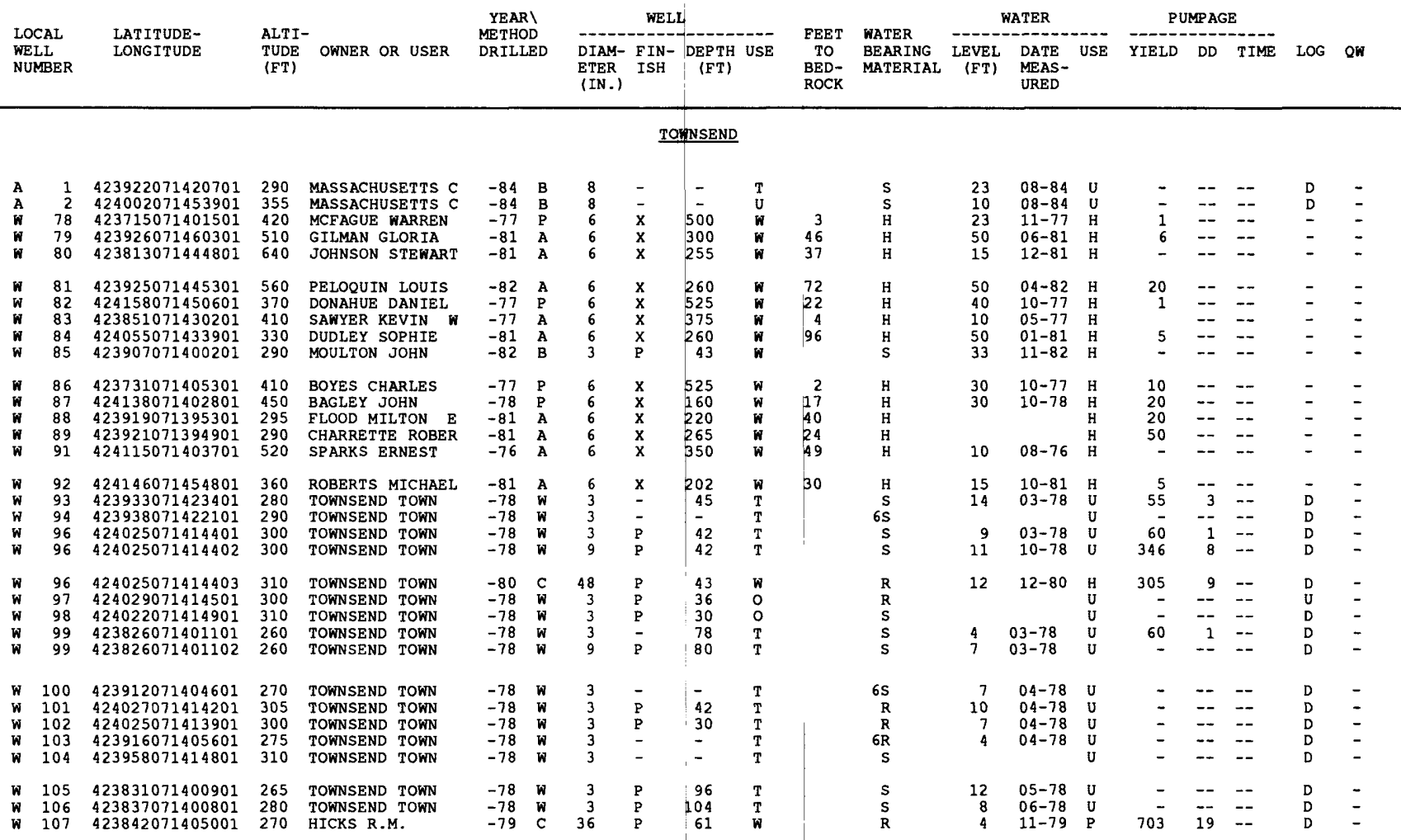


Table 2.--Logs of selected wells and borings

(Depths are given in feet below land surface)

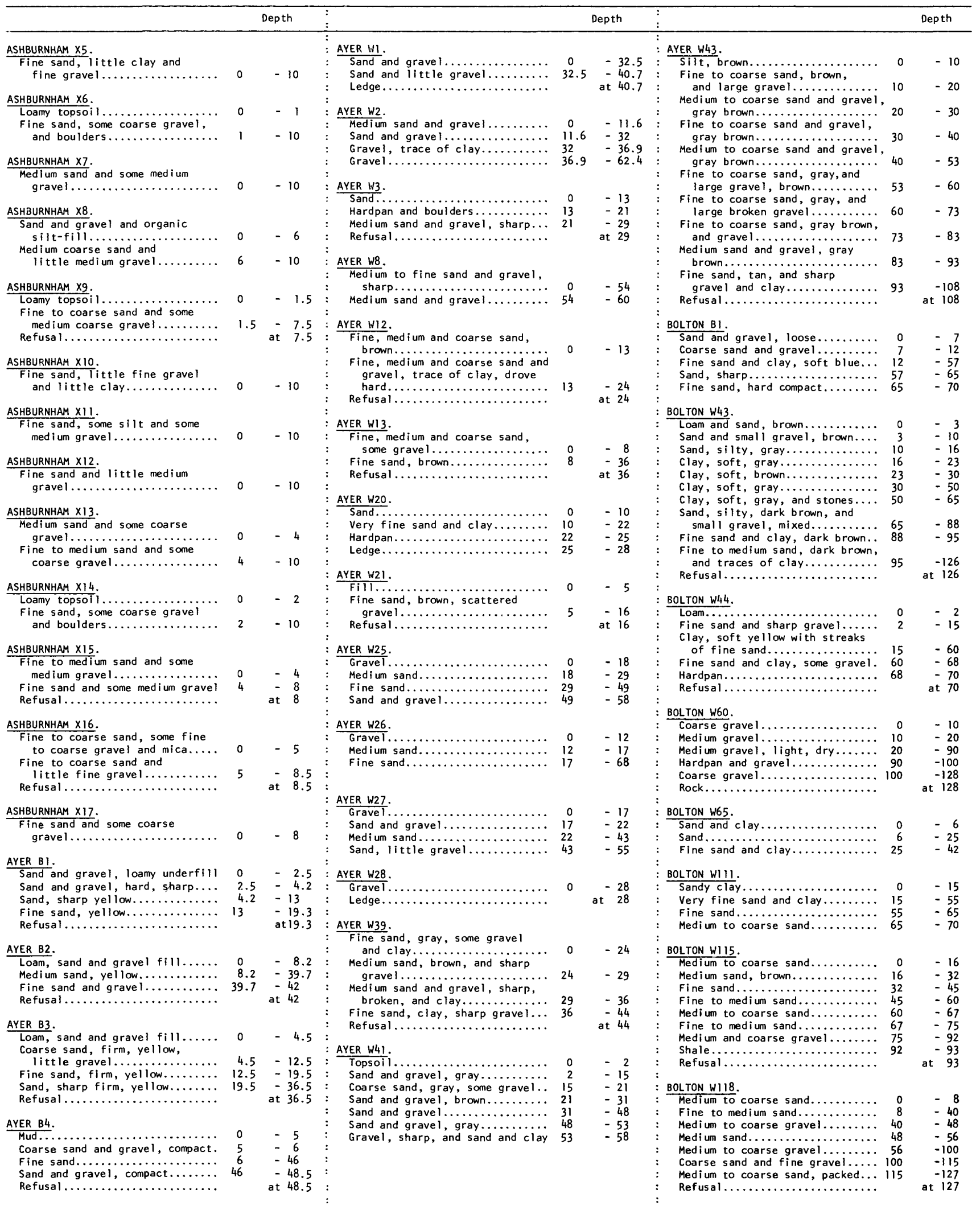


Table 2.--Logs of selected wells and borings (Continued)

(Depths are given in feet below land surface)

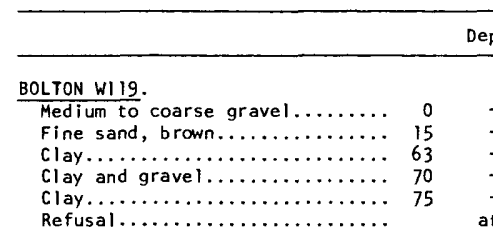

BOLTON WI20.

Fine sand, yellow, with st reaks

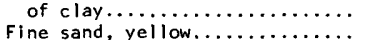
Fine sand, yellow............. 15 Medium sand, gray, and large

gravel................... 42

Sand, compact, gray, and gravel,

and some clay............... 53

Fine to medium sand, traces

of clay.................... 67

Fine to medium sand, gray........ $110 \quad-125$

Refusal.................... at 125

BOLTON WI2I.

Fine sand ................ 0.10

$\begin{array}{ll}\text { Medium to coarse gravel......... } 10 & 10 \\ \text { Clay.......................... } & 21\end{array}$

Medium to coarse gravel......... 48

CLINTON BI.

Mud, soft, black............. 0 - 3

Coarse sand, loose..................

and little clay, hard........ 15.5 at
Refusal....................

CLINTON B2.

Sand and gravel fill .

Sand and gravel fill.......... 0
Very fine sand with some clay... 32
Sand and gravel, compact......

CLINTON W5.

Gravel fill.

Medium coarse gravei.

Clay and sand....................

Gravel, sharp, packed............

Refusal...

CLINTON W6.

Sand and gravel.

$\ldots \ldots \ldots \ldots$

Refusal...

CLINTON W9.

Sand and sharp gravel, compact.

Sand and sharp gravel, compact.

Fine sand and gravel, gray...

Fine sand and clay, brown......... 32

Fine sand and gravel, gray....... 39

Fine sand and clay.................

CLINTON WII.

Sand clayey, yellow.

Sand, clayey, yellow........... 0

Peat.......................... very hard,

packed.....................

Gravel, water bearing.........
Coarse gravel, clean, water

bearing..................... 20

sand, very silty..................

CLINTON WI2.

Gravel and til

Clay and gravel.

Gravel, dirty.

CLINTON WI3.

Topsoil, loam...............

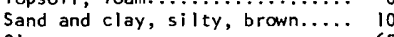

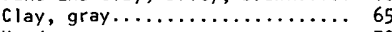
Hardpan.......

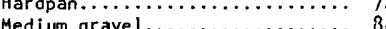

Medium gravel.....................

CLINTON WI4.

Topsoil and sand $. \ldots \ldots \ldots \ldots \ldots, 0$

Clay, gray...........

Clay and sand.

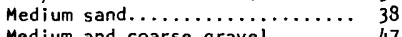

-8
-23
$-\quad 32$

$-10$

Depth

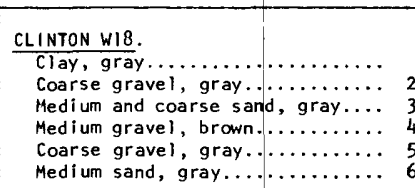

CLINTON WI9.

Medium and fine sand brown....

Medium and fine sand, brown.....
Fine and medium gravel, gray...

Fine and medium gravel, gray....

Ceat, brown...........

Coarse gravel, brown..............

Coarse gravel, gray, and

medium sand................ $84 \quad-100$

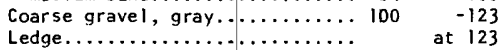

\section{CLINTON W20.}

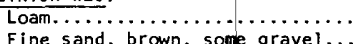

Fine sand, gray, and clay......

Fine sand, gray, and gravel.......

Fine to medium sand and gravel,

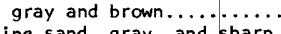

gravel............................. 69

Fine sand and sharp gravel.

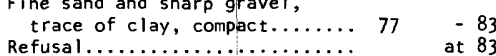

CLINTON W2I.

\section{Ash fill.}

Fine sand, yeliow.

Sand and clay, yellow.

Clay, silty, yellow.

Fine sand, gray, smalli sharp

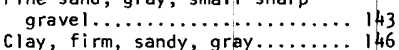

CLINTON W22.

Loam.................... 0

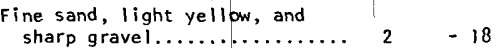

Sand, silty, yellow, and small

$\begin{array}{lll}\text { sharp gravel....................... } 18 & 27 & -27\end{array}$

Sand, silty gray, with streaks

of hard clay................ 72

CLINTON W23.

Loam........................ 00 -

Sand and gravel, medium compact.

Fine to medium sand and gravel.

fark brown

Fine sand and gravel, dark

brown......................

Refusal.................... at ${ }^{49}$

CLINTON X2.

Loam, some sand...

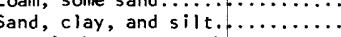

Gravel, heavy, gray brown.

Gravel, heavy, sharp, brown...

Gravel, brown, and clay...........

Clay and silt.................... 16

Clay and silt (sample)........... at

Refusal.................... at 55

CLINTON X4.

Loam and clay................ 0 . 2

Sand, gravel, and clay, hard

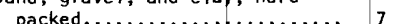

Hardpan.................... $12-16$

Refusal.................... at 16

FITCHBURG BI.

Silty sand, gravel and stones,

Coarse sand, gravel and stones,

hard.....................

Refusal on ledge or boulders...

FITCHBURG 82 .

Sand and gravel, some clay,

FITCHBURG WI.

Gravel and
Depth

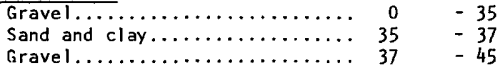

FITCHBURG W2.

FITCHBURG W3.

Gravel and boulders............ $0 \quad-12$

FITCHBURG W4.

Gravel and boulders............ 0 - 5

FITCHBURG U15

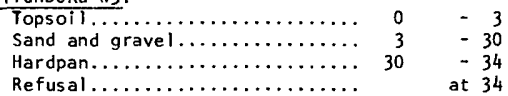

FITCHBURG W6.

Fine sand.

Clay, blue........................ 10.10

Hardpan.................... $12 \quad-13$

FITCHBURG W7.

Fine sand.

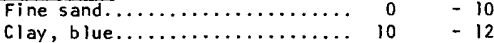

Hardpan.................. $12, \begin{aligned} & -14 \\ & 4\end{aligned}$

ITCHBURG WB.

Silt.........

$-19$

FITCHBURG W9.

Sand, dirty, fill $\ldots \ldots \ldots \ldots \ldots \ldots \ldots$

Coarse sand, dirty..............

Fine sand...

Fine sand and clay...

Coarse sand, water........... 20

Coarse gravel, water bearing.... 40

Hardpan..................... 60

Ledge..

FITCHBURG WIO

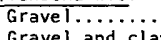

Gravel and clay............. 0

FITCHBURG WII.

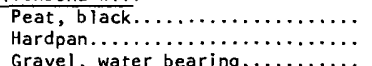

Hardpan..................... 50

Ledge...

FITCHBURG WI2.

Peat, black.

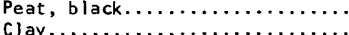

Sand, water bearing..............

Gravel, water bearing.

Gravel, water bearing.......... 45

Ledge...

FITCHBURG WI3.

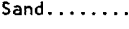

FITCHBURG WI4.

Sand...$\ldots \ldots \ldots$

Clay and sand.....................

Sand and gravel................. 26

Fine sand...................... 32

FITCHBURG WI6.

$\begin{array}{lll}\text { Loam.......................... } & 0 & -2 \\ \text { Sand and gravel, compact...... } & 2 & -14\end{array}$

Fine to medium sand, brown,

some gravel............... 14

Medium sand and gravel, brown... 45

FITCHBURG WI9.

75 gallons per minute..........

135 gallons per minute...........

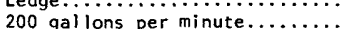

FITCHBURG W22.

Sand and $g$ ras

Hardpan.
Refusal.

$-13.5$

FITCHBURG W23.

Fopsoil.....

Fine sand...............

Big rocks...

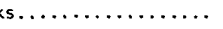

Fine sand and gravel..............

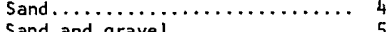

Sand and gravel $\ldots \ldots \ldots \ldots \ldots \ldots \ldots$
Gravel and rocks............. 50
$-5$

-10
-15
-20

-
$-\quad 20$
$-\quad 35$

-40
-60

- 66.5

at 66.5

-40
-60

$\begin{array}{cc}- & 3 \\ - & 8 \\ - & 50 \\ - & 58.5 \\ \text { at } 58.5\end{array}$

-5
-15
-15

$-45$

-60
-66

at 66

$-40$

- 18

-26
$-\quad 32$
$-\quad 40$

40

-45
-48

at 25

at 43

at 45
at 50

-14
$-\quad 38$
-38

at 38

-5
$=10$
-20
$=30$
$=40$
$=50$
-55
-61 
Table 2.--Logs of selected wells and borings (Continued)

(Depths are given in feet below land surface)

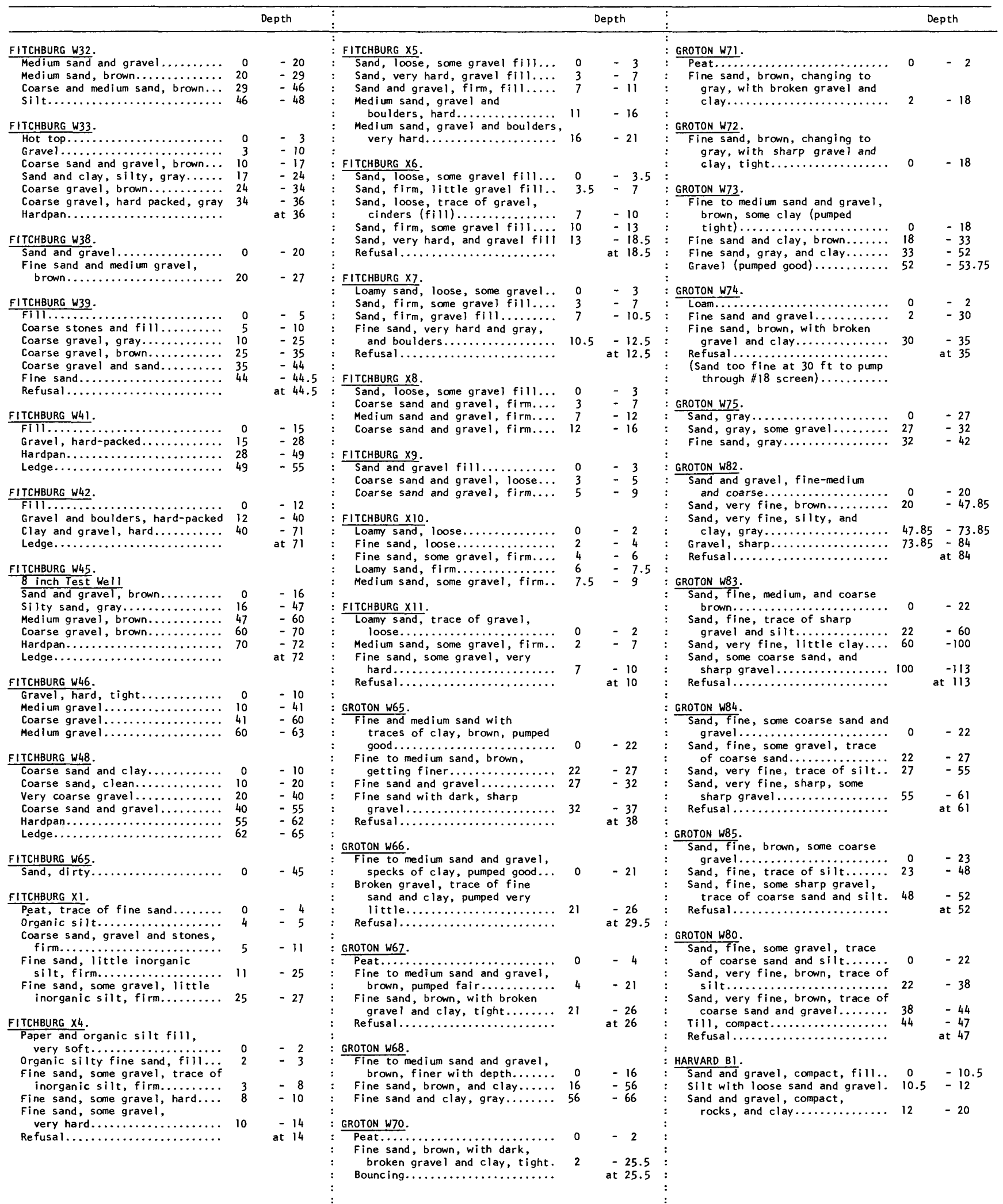


Table 2.--Logs of selected wells and borings (Continued)

(Depths are given in feet below land surface)

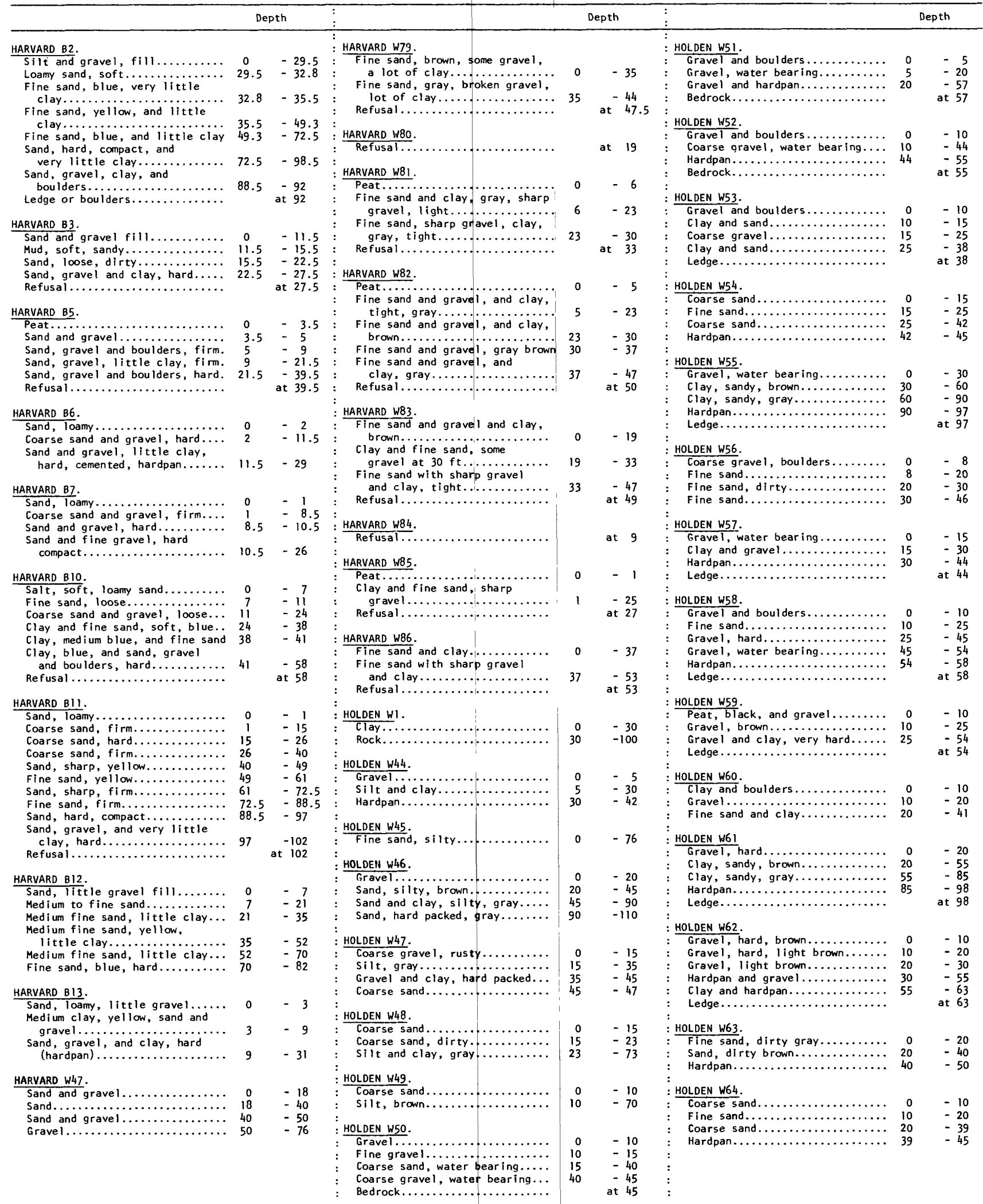


Table 2.--Logs of selected wells and borings (Continued)

(Depths are given in feet below land surface)

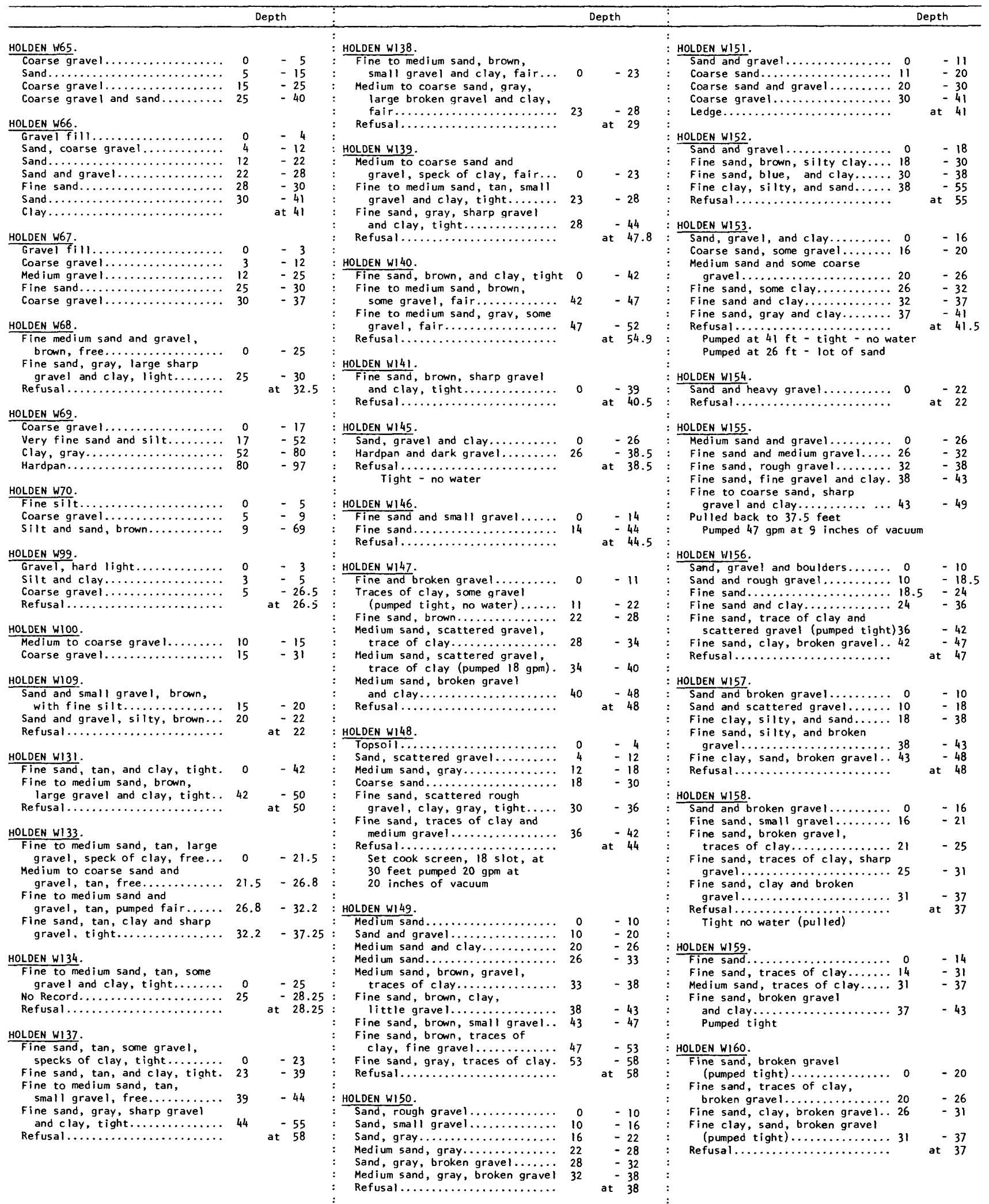


Table 2.--Logs of selected well's and borings (Continued)

(Depths are glven in feek below land surface)

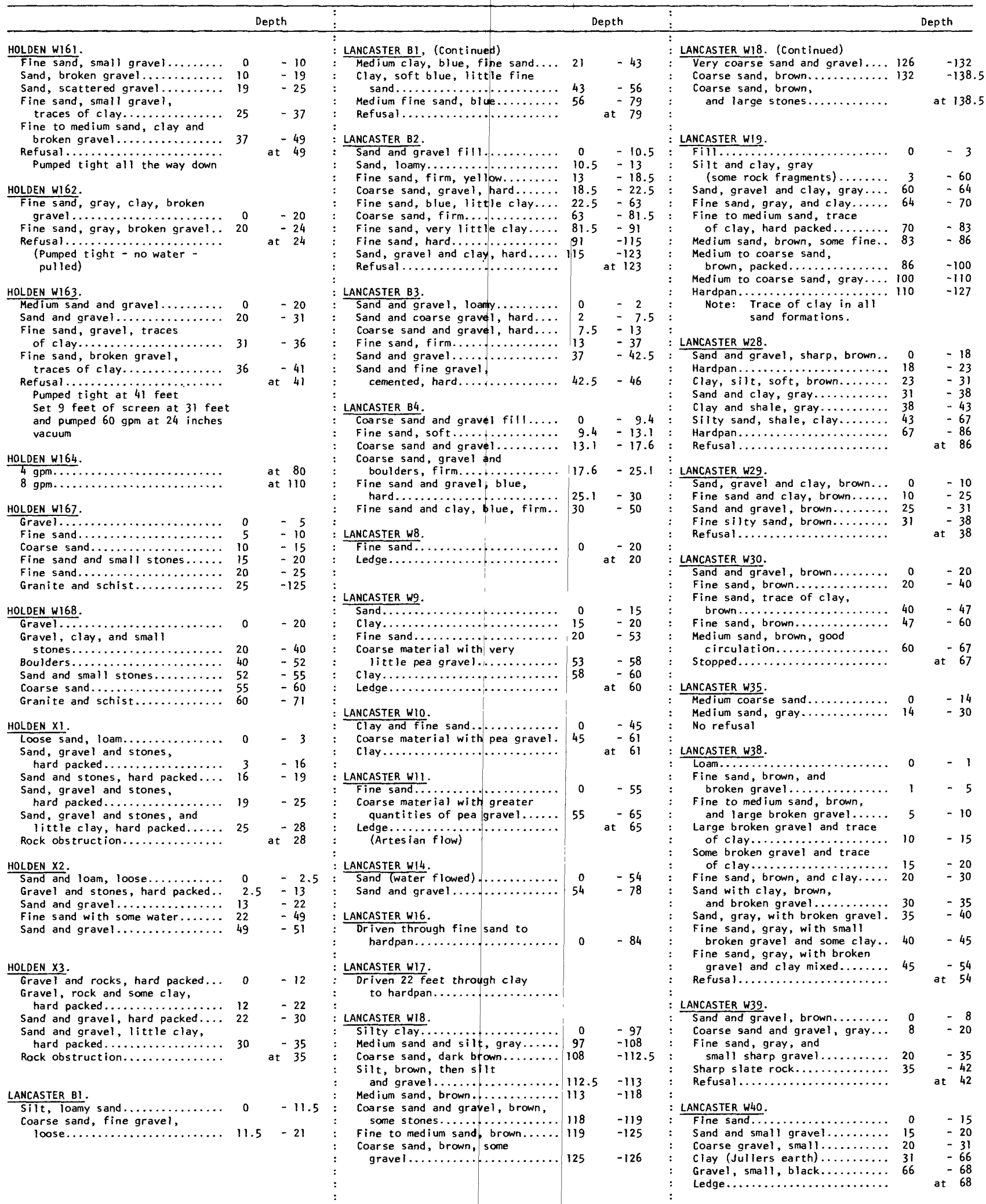


Table 2.--Logs of selected wells and borings (Continued)

(Depths are given in feet below land surface)

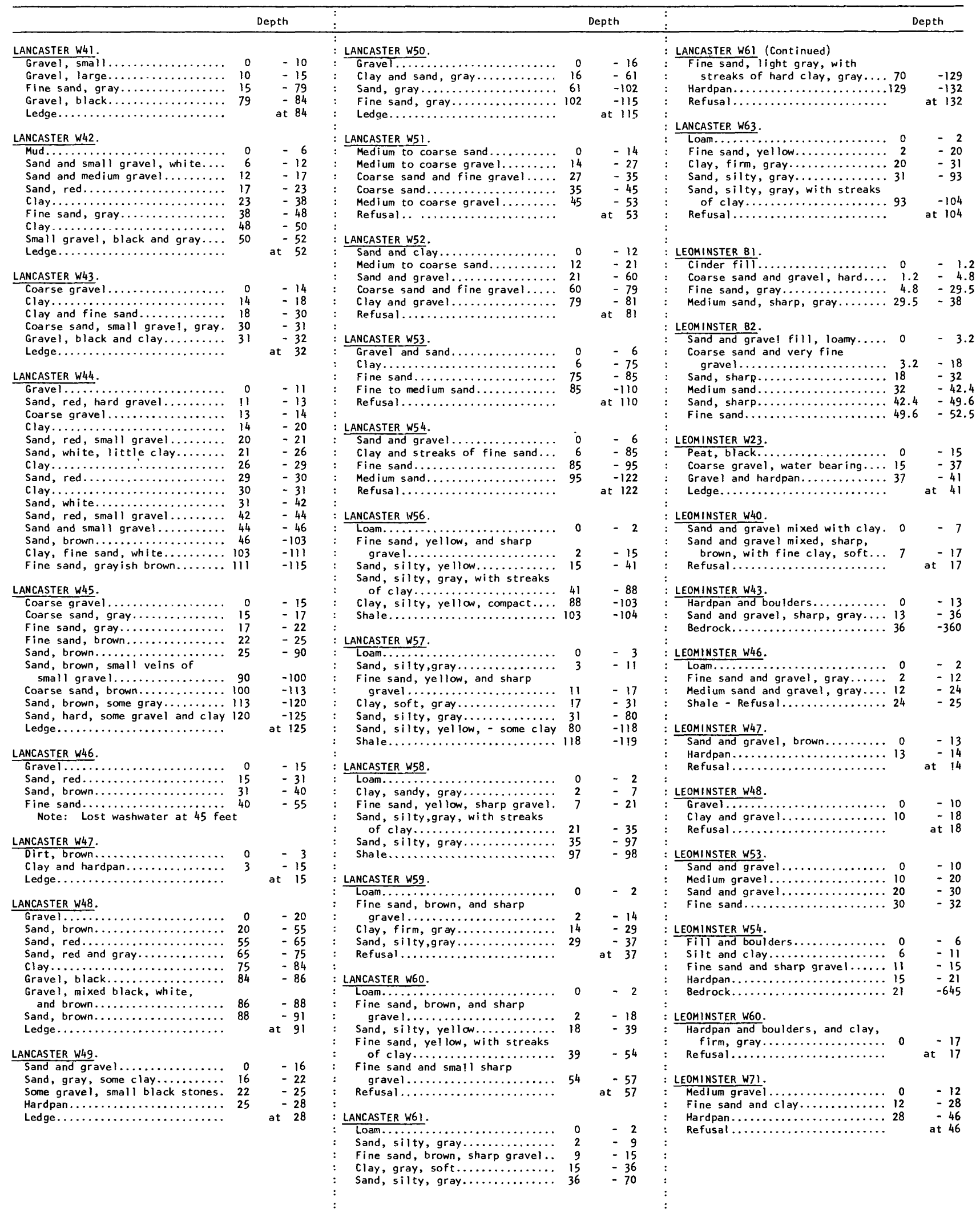


Table 2.--Logs of selected whells and borings (Continued)

(Depths are given in feet be low land surface)

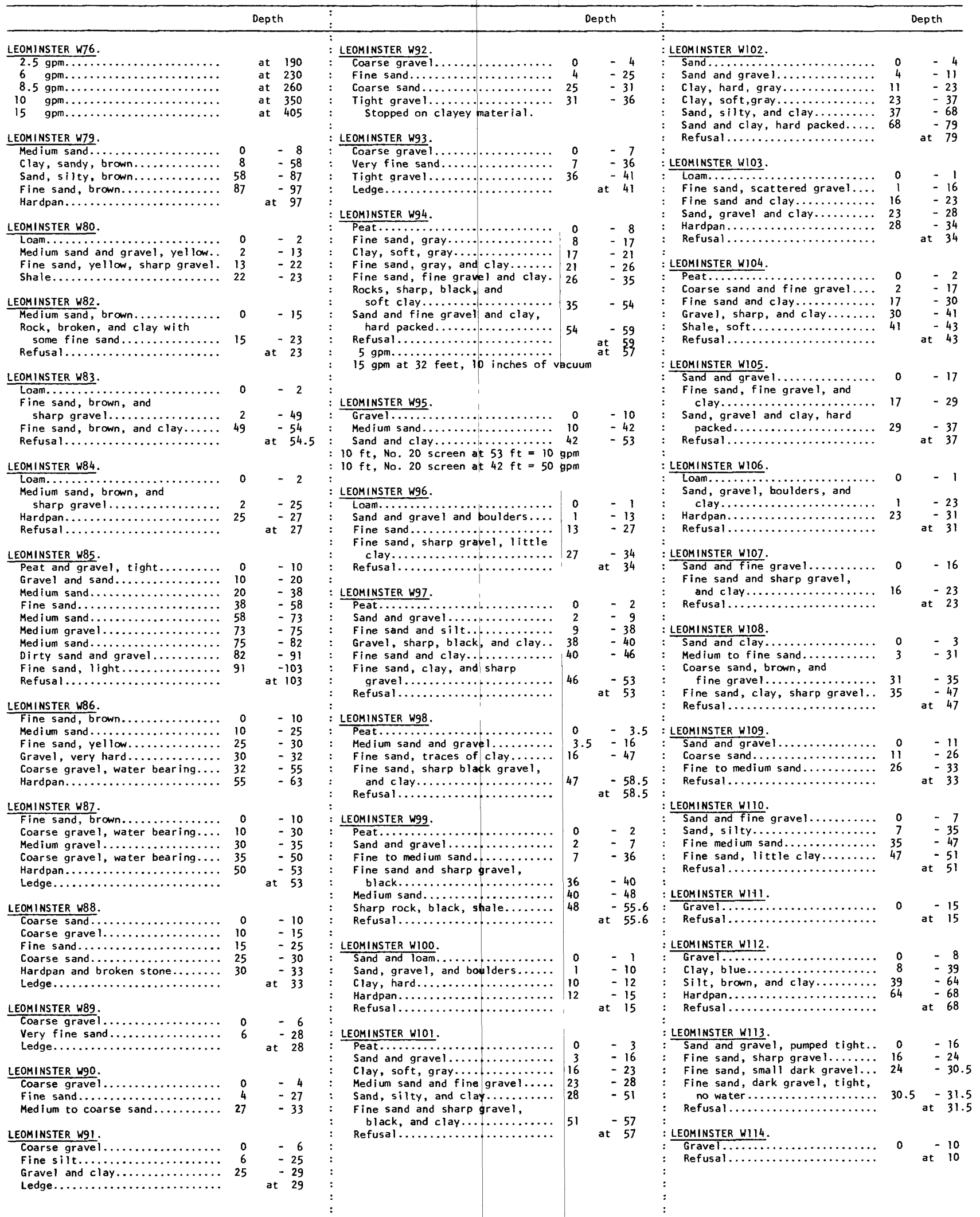


Table 2.--Logs of selected wells and borings (Continued)

(Depths are given in feet below land surface)

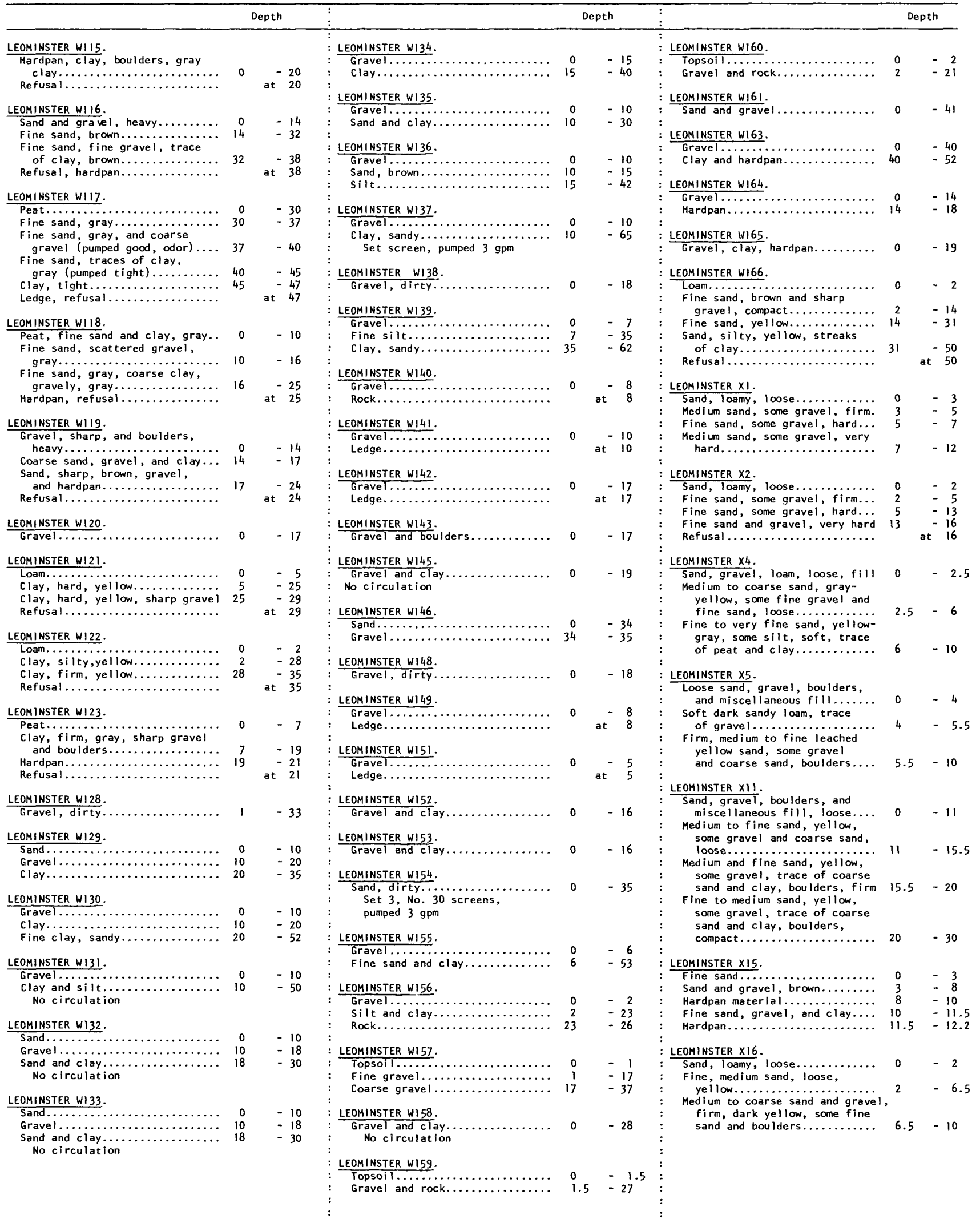


Table 2.--Logs of selected wells and borings (Continued)

(Depths are given in feet below land surface)

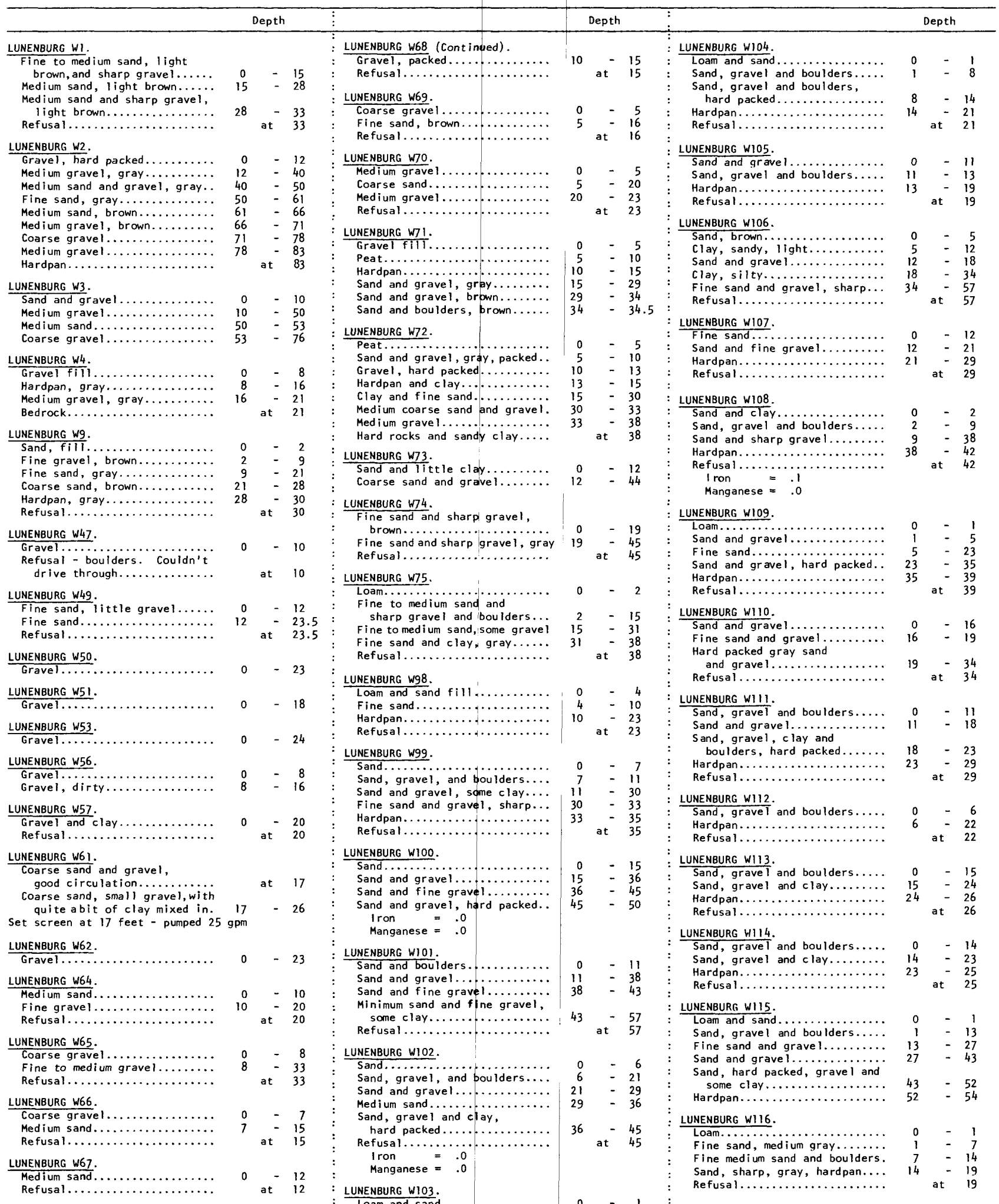

LUNENBURG W68.

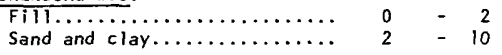

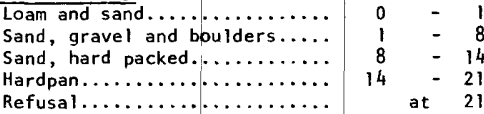


Table 2.--Logs of selected well ls and borings (Continued)

(Depths are given in feet below land surface)

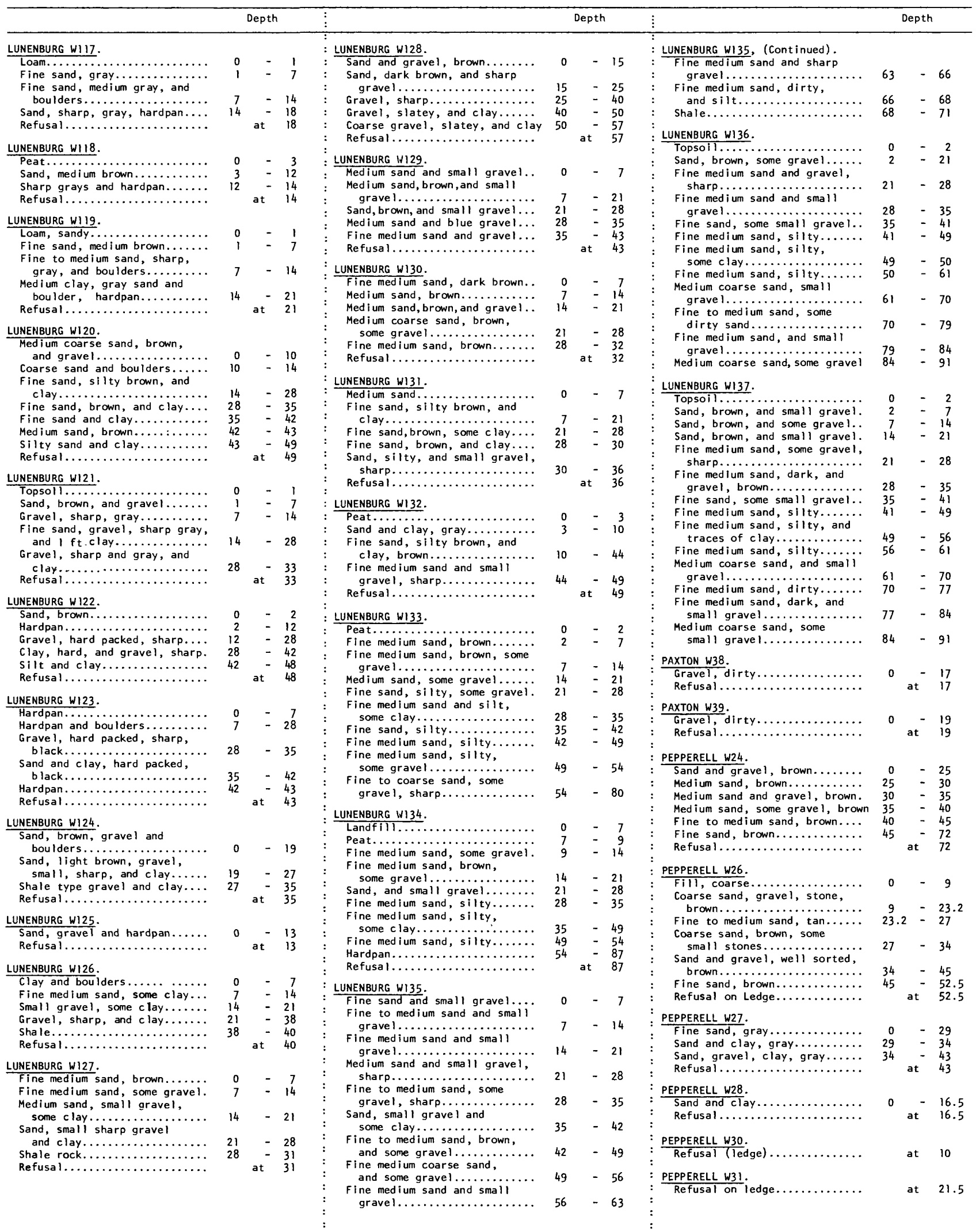


Table 2.--Logs of selected wells and borings (Continued)

(Depths are given in feet below land surface)

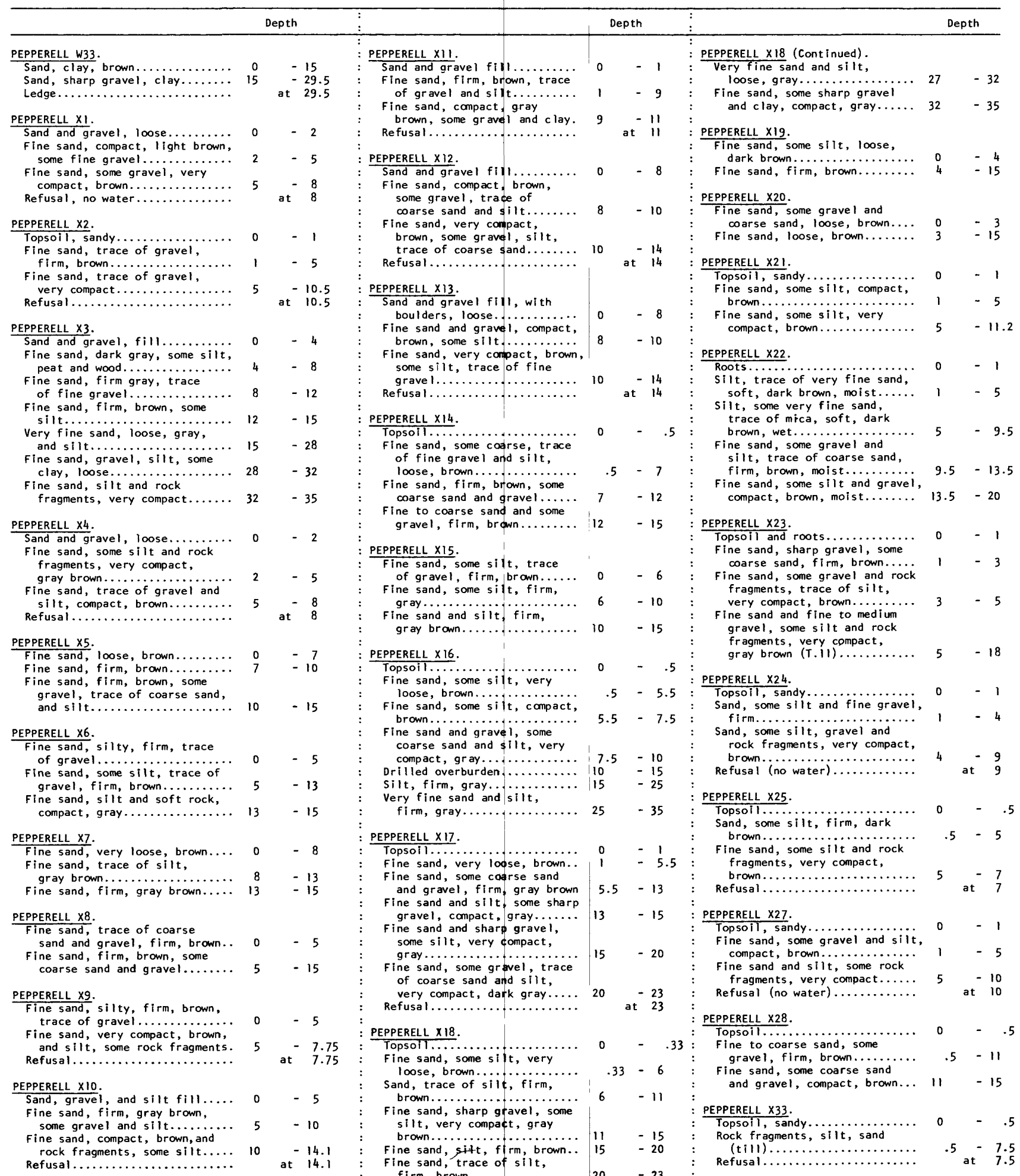


Table 2,--Logs of selected wells and borings (Continued)

(Depths are given in feet below land surface)

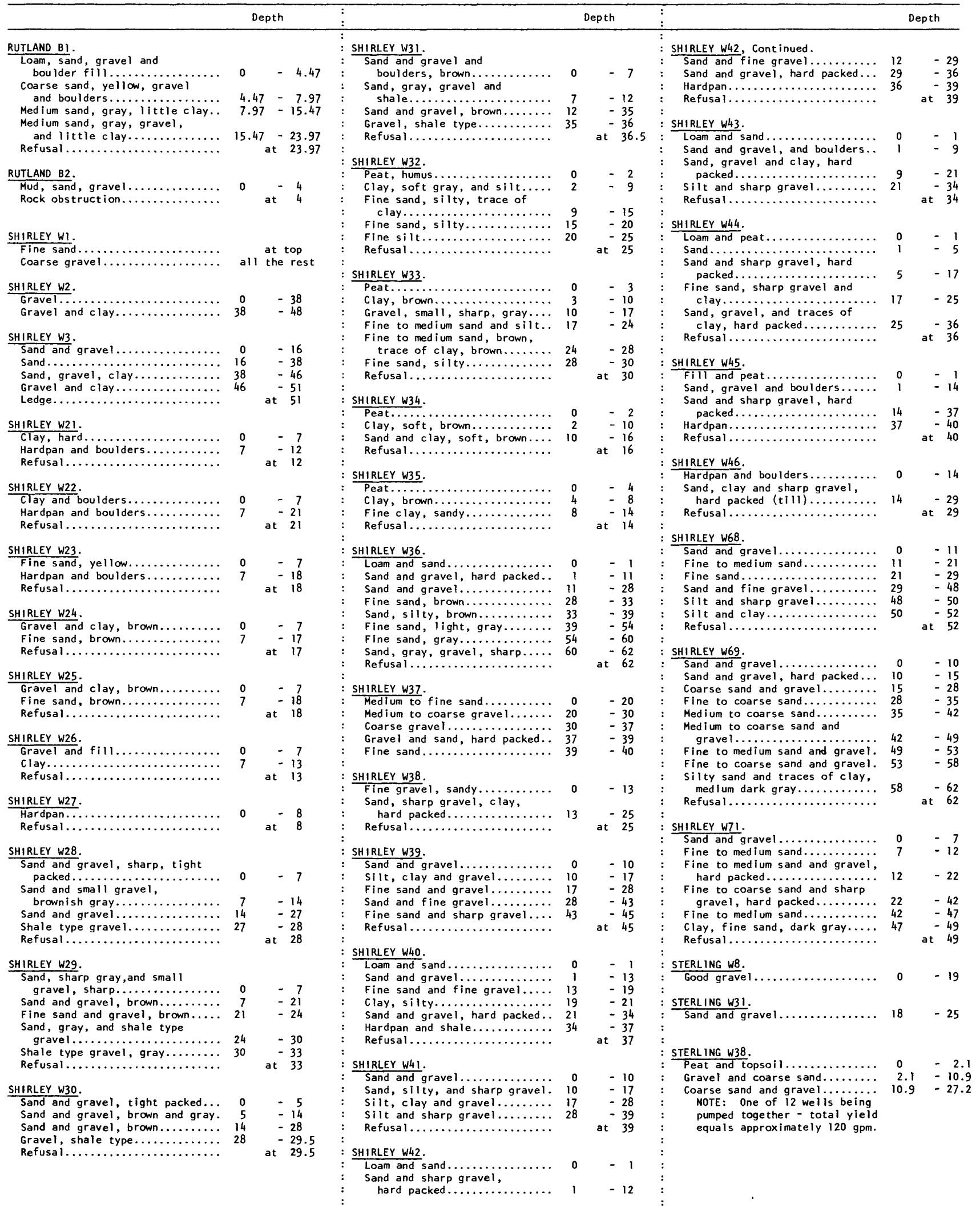


Table 2.--Logs of selected wel is and borings (Continued)

(Depths are given in feet below land surface)

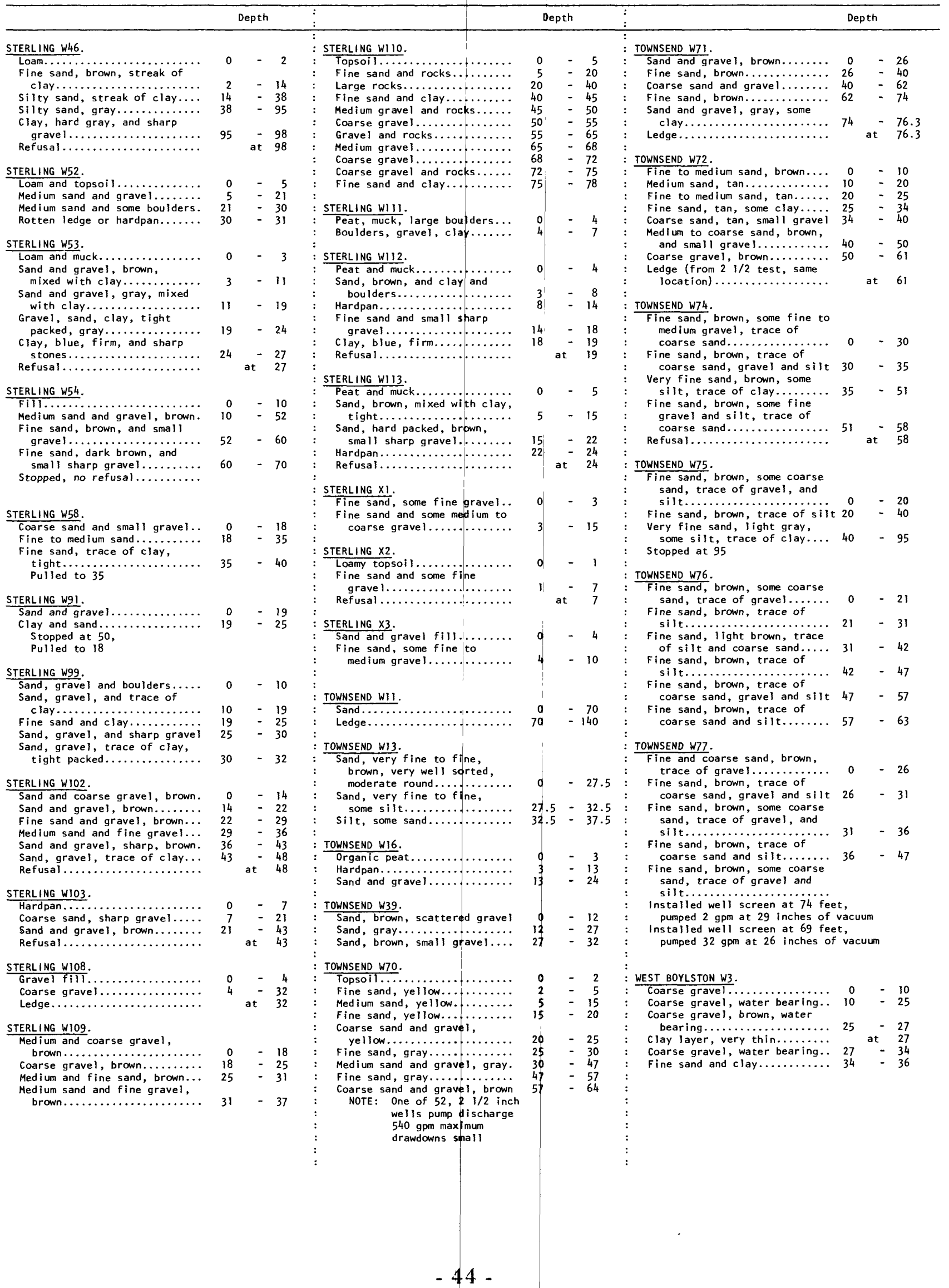


Table 2.--Logs of selected wells and borings (Continued)

(Depths are given in feet below land surface)

\begin{tabular}{|c|c|c|c|c|c|c|c|c|c|c|c|c|c|}
\hline & \multicolumn{3}{|c|}{ Depth } & $\vdots$ & & & Depth & & 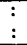 & & & pth & \\
\hline WEST BOYLSTON W4. & & & & & WEST BOYLSTON XI. & & & & $\vdots$ & WESTMINSTER W40. & & & \\
\hline Silt and loam... & 0 & - & -10 & $:$ & Quartzite...... & & at & 32 & : & Hardpan and grave $1 \ldots \ldots \ldots \ldots \ldots$ & 0 & - & 5 \\
\hline Clay and small stones.......... & 10 & - & -20 & : & Penetration -9.8 feet & & & & : & Hardpan and boulders........... & 5 & - & - 10 \\
\hline Clay and gravel.............. & 20 & - & -35 & : & & & & & $:$ & Hardpan..................... & 10 & - & - 12 \\
\hline Medium gravel.............. & 35 & & -40 & : & & & & & : & Refusal...................... & & at & $=12$ \\
\hline Coarse gravel............... & 40 & & -45 & : & & & & & : & & & & \\
\hline Coarse gravel and stones...... & 45 & & -50 & & WESTMINSTER BI. & & & & $:$ & & & & \\
\hline 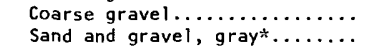 & $\begin{array}{l}50 \\
58\end{array}$ & & $\begin{array}{r}58 \\
-\quad 65\end{array}$ & $\vdots$ & $\begin{array}{l}\text { Peat, soft........................ } \\
\text { Silt, sandy, very loose, }\end{array}$ & I & & 23 & $:$ & $\frac{\text { WESTMINSTER W } 42}{\text { Sand, medium }}$ and coarse, & & & \\
\hline $\begin{array}{l}\text { Refusal* } \ldots \ldots \ldots \ldots \ldots \ldots \ldots \\
\quad \text { *from } 8 \text { inch test weli, same }\end{array}$ & & at & 65 & $\vdots$ & $\begin{array}{l}\text { wet, and trace of gravel.... } \\
\text { Sand, medium gray, some }\end{array}$ & 23 & - & 31.5 & $\vdots$ & $\begin{array}{l}\text { appears to have rust........ } \\
\text { Sand, med ium, some gravel }\end{array}$ & 0 & - & - 10 \\
\hline location & & & & $\vdots$ & $\begin{array}{l}\text { gravel, trace of clay, } \\
\text { and boulders................ }\end{array}$ & 31.5 & $5-$ & 39 & $:$ & $\begin{array}{l}\text { (pumped } 45 \mathrm{gpm}) \ldots \ldots \ldots \ldots \ldots \\
\text { Sand, med ium }(60 \mathrm{gpm},\end{array}$ & 10 & - & - 15 \\
\hline WEST BOYLSTON W5. & & & & : & Refusal...................... & & at & 39 & : & water clearer) $\ldots \ldots \ldots \ldots \ldots$ & 15 & - & - 20 \\
\hline 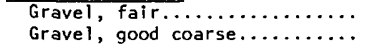 & $\begin{array}{r}0 \\
30\end{array}$ & & $-\begin{aligned} 30 \\
-\quad 48\end{aligned}$ & : & WESTMINSTER B2. & & & & $:$ & $\begin{array}{l}\text { Sand, medium ( } 36 \mathrm{gpm} \text {, began } \\
\text { driving harder)............. }\end{array}$ & 20 & - & \\
\hline 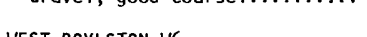 & & & & & Medium sand, brown, trace of & & & & $:$ & Boulders.................... & $-\infty$ & at & 25 \\
\hline WEST BOYLSTON W6. & & & & & silt, trace of medium & & & & : & & & & \\
\hline Coarse gravel......... & 0 & & -30 & : & gravel $\ldots \ldots \ldots \ldots \ldots \ldots \ldots$ & 0 & - & 4 & : & & & & \\
\hline $\begin{array}{l}\text { Sand and gravel} \ldots \ldots \ldots \ldots \ldots \ldots \\
\text { Clay, gray } \ldots \ldots \ldots \ldots \ldots \ldots\end{array}$ & $\begin{array}{l}30 \\
60\end{array}$ & & $\begin{array}{r}60 \\
-\quad 80\end{array}$ & $:$ & $\begin{array}{l}\text { Fine to medium sand, little } \\
\text { silt, trace of coarse }\end{array}$ & & & & $:$ & $\frac{\text { WESTMINSTER W46. }}{\text { Sand, med i } 4 \text { and coarse, }}$ & & & \\
\hline Hardpan and ledge............. & & at & 80 & $\vdots$ & $\begin{array}{c}\text { gravel..................... } \\
\text { Fine sand, brown, little silt, }\end{array}$ & 4 & - & 10 & $\vdots$ & 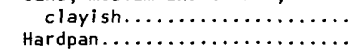 & $\begin{array}{r}0 \\
15\end{array}$ & & $\begin{array}{l}15 \\
20\end{array}$ \\
\hline WEST BOYLSTON WI9. & & & & & trace of decomposed mica & & & & : & & & & \\
\hline Clay, sandy...... & 0 & - & -21 & : & $\begin{array}{l}\text { schist.............................. } \\
\text { Mica schist, gray, with }\end{array}$ & 10 & - & 12 & $:$ & WESTMINSTER W47. & & & \\
\hline WEST BOYLSTON W2O. & & & & : & quartz seams, medium to & & & & : & Medium and coarse sand........ & 0 & - & 10 \\
\hline Gravel and boulders........... & 0 & - & 21 & $\vdots$ & $\begin{array}{l}\text { hard } \ldots \ldots \ldots \ldots \ldots \ldots \ldots \ldots \\
\text { Mica schist, gray, with }\end{array}$ & 12 & - & 17 & $:$ & $\begin{array}{l}\text { Coarse sand, some clay........ } \\
\text { Sand, clayish, and some }\end{array}$ & 10 & - & 15 \\
\hline WEST BOYLSTON W21. & & & & : & quartz seams, soft........... & 17 & - & 22 & : & fine gravel........... & 15 & - & 20 \\
\hline Gravel and boulders.. & 0 & - & -42 & : & WESTMINSTER B3. & & & & $\vdots$ & $\begin{array}{l}\text { Coarse sand and fine gravel... } \\
\text { Medium and coarse sand, }\end{array}$ & 20 & - & 25 \\
\hline WEST BOYLSTON W22. & & & & : & Fine to medium sand, brown, & & & & : & clayish................ & 25 & - & 30 \\
\hline Sand and gravel.. & 0 & & -16 & : & topsoil $1 \ldots \ldots \ldots \ldots \ldots \ldots$ & 0 & - & 2 & : & Broken gravel, clayish sand... & 30 & - & 33 \\
\hline 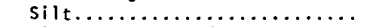 & 16 & & -25 & : & Fine to coarse sand and gravel, & & & & : & Rock....................... & & at & 33 \\
\hline silt and trace of clay.......... & 25 & & -30 & : & brown, boulder chips........ & 2 & - & 8 & : & & & & \\
\hline 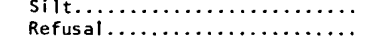 & 30 & & $\begin{array}{r}68 \\
68\end{array}$ & $:$ & $\begin{array}{l}\text { Fine to medium sand, brown, } \\
\text { cobbles, some silt.......... }\end{array}$ & & & & $:$ & & & & \\
\hline Retusal..................... & & & & & Fine to medium sand and & 8 & - & 15 & $\vdots$ & 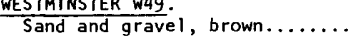 & 0 & - & 5 \\
\hline WEST BOYLSTON W23. & & & & : & silt, brown gray, trace of & & & & : & Mardpan $\ldots \ldots \ldots \ldots \ldots \ldots \ldots \ldots$ & 5 & - & 7 \\
\hline Loam............. & 0 & - & 2 & : & gravel, boulder chips, & & & & : & Refusal.................... & & at & 7 \\
\hline Clay...$\ldots \ldots \ldots \ldots \ldots$ & 2 & & -4 & : & mica schist............. & 15 & - & 19.7 & : & & & & \\
\hline $\begin{array}{l}\text { Gravel, sandy, hard packed.... } \\
\text { Sand and gravel, brown, and }\end{array}$ & 4 & & -10 & $\vdots$ & Refusal................. & & at & 19.7 & $:$ & $\frac{\text { WESTMINSTER W50 }}{\text { Sand and grave } 1 . . \ldots \ldots \ldots}$ & & & \\
\hline 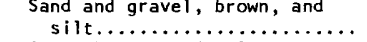 & 10 & 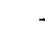 & 25 & : & & & & & $\vdots$ & Sand and gravel, with clay..... & $\begin{array}{r}0 \\
15\end{array}$ & $\overline{-}$ & $\begin{array}{l}15 \\
25\end{array}$ \\
\hline Sand, brown, and silt......... & 25 & & -40 & & WESTMINSTER W4. & & & & : & Coarse gravel and sand, with & & & \\
\hline Sand, fine to medium, brown, & & & & & 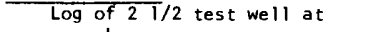 & & & & $:$ & 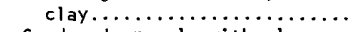 & 25 & & \\
\hline and silt $\ldots \ldots \ldots \ldots \ldots \ldots$ & 40 & & -45 & : & pump house & & & & $:$ & Sand and gravel, with clay.... & 30 & & \\
\hline Sand, fine, brown, and silt... & 45 & & -58 & & Sand and scattered gravel..... & 0 & - & 22 & : & Refusal $\ldots \ldots \ldots \ldots \ldots \ldots \ldots$ & & at & 40 \\
\hline Sand, fine to coarse, brown... & $\begin{array}{l}58 \\
60\end{array}$ & & 60 & $:$ & $\begin{array}{l}\text { Fine sand............................... } \\
\text { Fine to medium sand, sharp }\end{array}$ & 22 & - & 33 & $\vdots$ & & & & \\
\hline & & & & $\vdots$ & gravel and some clay........ & 33 & - & 44.8 & : & WESTMINSTER W5I. & & & \\
\hline WEST BOYLSTON W24. & & & & : & Refusal.................. & & at & 44.8 & : & Sand and boulders, brown.. & 0 & - & 5 \\
\hline Loam............. & 0 & & - 2 & : & $5 \mathrm{gpm}$ at 38 feet & & & & : & Sand and gravel, with clay.... & 5 & - & 10 \\
\hline Silt.. & 2 & & 20 & : & $45 \mathrm{gpm}$ at 19 feet with & & & & : & Sand and clay mixed............. & 10 & - & 15 \\
\hline Sand, sharp, gravel and silt.. & 20 & & -30 & : & 10 inches of vacuum & & & & & 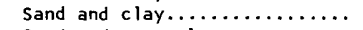 & 15 & - & \\
\hline Sand, fine, gravel and silt... & 30 & & 40 & : & & & & & $:$ & Sand and gray clay............ & 30 & & \\
\hline Sand, fine, brown.............. & 40 & & -45 & : & & & & & & Sand and gravel, with clay.... & 35 & - & \\
\hline Sand, fine to medium, brown... & 45 & & -50 & : & WESTMINSTER W7. & & & & ; & Gravel, sharp, with clay....... & 40 & - & 41 \\
\hline Sand, medium to coarse, brown. & 50 & & -64 & : & Clay, blue $\ldots \ldots \ldots \ldots \ldots \ldots$ & 0 & & 5 & $:$ & Refusal.................... & & at & 41 \\
\hline $\begin{array}{l}\text { Sand, fine, brown, and silt... } \\
\text { Refusal }\end{array}$ & & & $\begin{array}{r}81.5 \\
815\end{array}$ & : & Boulders, flat................ & 5 & ${ }^{-}$ & 6 & $:$ & & & & \\
\hline Rerusal........................... & & & & $:$ & Quicksand, water-bearing...... & & & & $\vdots:$ & $\frac{\text { WESTMINSTER W52 }}{\text { FIII }}$ & 0 & - & 5 \\
\hline & & & & : & & & & & : & Clay and peat... & 5 & & 10 \\
\hline WEST BOYLSTON W25. & & & & & WESTMINSTER W25. & & & & : & clay and sand................. & 10 & - & 15 \\
\hline Sand and gravel.. & 0 & & & : & Clay...$\cdots \ldots \ldots$ & 0 & & 65 & : & Sand and gray clay............. & 15 & - & 25 \\
\hline Medium gravel.... & 40 & & 57 & : & Gravel. & 65 & & 108 & : & Coarse sand and clay............ & 25 & - & 30 \\
\hline Sand and grave $1, \ldots \ldots \ldots \ldots \ldots$ & 57 & & -91 & : & Rock....................... & 108 & & 190 & & Gravel, sand, and clay, mixed. & 30 & & 35 \\
\hline Coarse sand $\ldots \ldots \ldots \ldots \ldots \ldots$ & 91 & & -101 & : & & & & & : & Sand and gravel with clay..... & 35 & - & 40 \\
\hline Sand and grave $1 \ldots \ldots \ldots \ldots \ldots$ & 101 & & 104 & & WESTMINSTER W28. & & & & : & Fine sand and clay.............. & 40 & - & 45 \\
\hline Coarse sand $\ldots \ldots \ldots \ldots \ldots \ldots \ldots$ & 104 & & 107 & : & Fine sand..... & 0 & & 120 & : & Sand and clay............... & 45 & - & 50 \\
\hline Sand and gravel.................. & 107 & & 110 & : & Clay, soft, brown.. & 120 & & 130 & : & Refusal $\ldots \ldots \ldots \ldots \ldots \ldots \ldots$ & & at & 50 \\
\hline Coarse sand $\ldots \ldots \ldots \ldots \ldots \ldots \ldots$ & 110 & & 112 & : & Gravel, good....... & 130 & & 143 & : & & & & \\
\hline Fine sand ${ } \ldots \ldots \ldots \ldots \ldots \ldots \ldots$ & 114 & & 124 & : & Rock $\ldots \ldots \ldots \ldots \ldots \ldots \ldots \ldots$ & 143 & & 153 & : & WESTMINSTER W55. & & & \\
\hline Sand and gravel, dirty*...... & 124 & & 137 & : & Well pulled to 143 & & & & : & Boulders and peat.... & 0 & & 5 \\
\hline Ledge $^{*} \ldots \ldots \ldots \ldots \ldots \ldots \ldots \ldots$ & & at & 137 & : & & & & & : & Peat and clay $\ldots \ldots \ldots \ldots \ldots \ldots$ & 5 & - & 10 \\
\hline tFrom 8 inch test well, & & & & : & & & & & : & Clay and hardpan.............. & 10 & & 15 \\
\hline same location & & & & : & & & & & : & Hardpan and boulders........... & 15 & - & 19 \\
\hline & & & & : & & & & & : & Refusal.................... & & at & 19 \\
\hline & & & & : & & & & & : & & & & \\
\hline & & & & : & & & & & : & & & & \\
\hline & & & & : & & & & & : & & & & \\
\hline & & & & : & & & & & : & & & & \\
\hline & & & & : & & & & & : & & & & \\
\hline & & & & : & & & & & : & & & & \\
\hline & & & & : & & & & & : & & & & \\
\hline & & & & : & & & & & : & & & & \\
\hline & & & & : & & & & & : & & & & \\
\hline & & & & : & & & & & : & & & & \\
\hline & & & & : & & & & & : & & & & \\
\hline & & & & : & & & & & : & & & & \\
\hline & & & & : & & & & & $:$ & & & & \\
\hline
\end{tabular}


Table 2.--Logs of selected wells and borings, (Continued)

(Depths are given in feet below land surface)

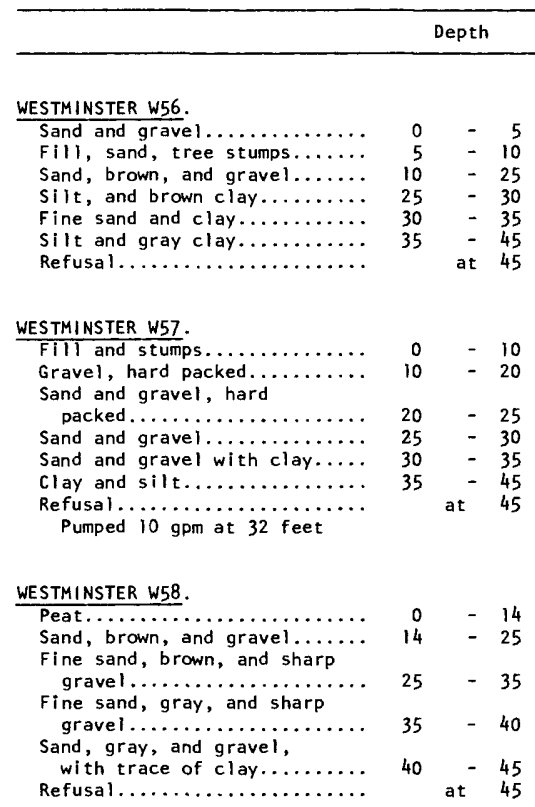

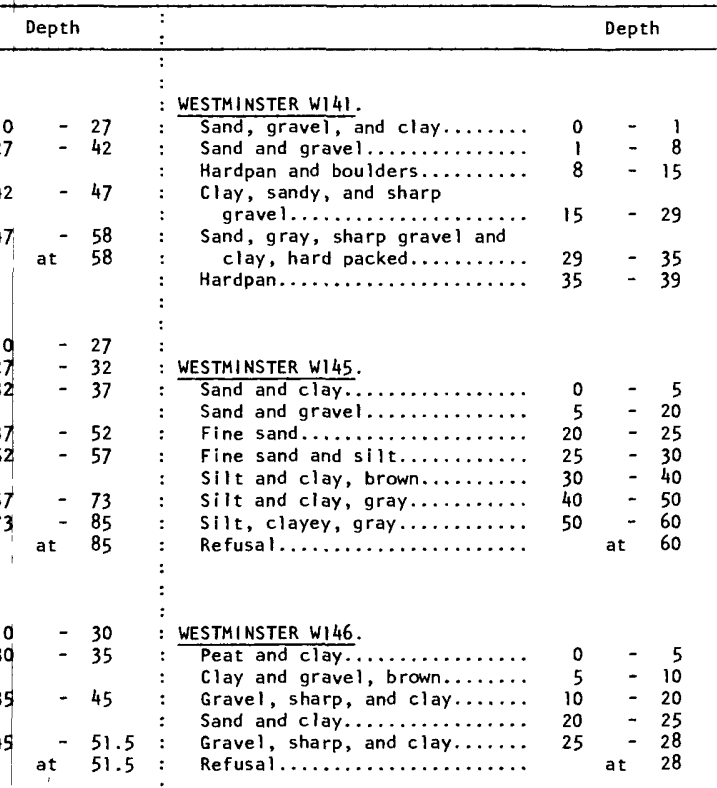


Table 2A. -- Logs of selected wells and borings

(Depths are given in feet below land surface)

\begin{tabular}{|c|c|c|}
\hline \multicolumn{3}{|l|}{ GROTON A1 } \\
\hline Topsoil............. & 0 & 2 \\
\hline Coarse to very coarse sand.... & 2 & 7 \\
\hline Fine sand.............. & 7 & -37 \\
\hline silt and clay $\ldots \ldots \ldots \ldots \ldots \ldots$ & 47 & -60 \\
\hline Very fine sand and silt....... & 60 & -75 \\
\hline Very fine sand with fine gravel & 75 & -77 \\
\hline 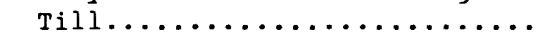 & 77 & -85 \\
\hline 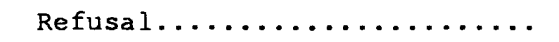 & & at 85.5 \\
\hline LANCASTER A1 & & \\
\hline Mud and loam............... & 0 & 2 \\
\hline Gravel............. & 2 & 4.5 \\
\hline Coarse to very coarse sand.... & 4.5 & -12 \\
\hline Clay, silt, and sand......... & 12 & -50 \\
\hline Very fine sand and silt....... & 50 & -75 \\
\hline Fine to coarse sand........... & 75 & -99 \\
\hline Cobbles............. & 99 & -100 \\
\hline 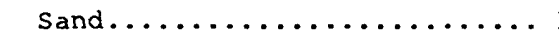 & 100 & -122 \\
\hline LANCASTER A2 & & \\
\hline Fill $\ldots \ldots \ldots \ldots \ldots \ldots \ldots \ldots \ldots$ & 0 & -3 \\
\hline Very coarse sand and & & \\
\hline fine gravel....... & 3 & -40 \\
\hline Fine to coarse sand.......... & 40 & -55 \\
\hline 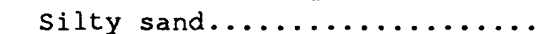 & 55 & -75 \\
\hline silt and clay..... & 75 & -95 \\
\hline Fine sand, some silt......... & 95 & -122 \\
\hline
\end{tabular}

\begin{tabular}{|c|c|c|}
\hline ANCASTER W88 & & \\
\hline Topsoil $\ldots \ldots \ldots \ldots \ldots \ldots \ldots$ & 0 & 2.5 \\
\hline 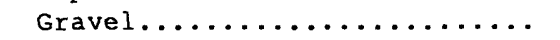 & 2.5 & 4 \\
\hline 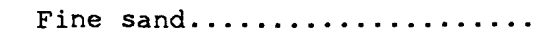 & & -117 \\
\hline STERLING W150 & & \\
\hline 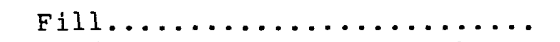 & 0 & -20 \\
\hline Sand with some fine gravel.... & 20 & -92 \\
\hline 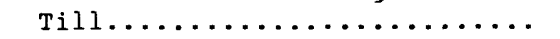 & 92 & -94.5 \\
\hline 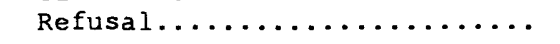 & & at 94.5 \\
\hline STERLING W151 & & \\
\hline 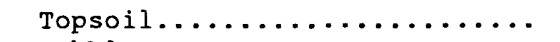 & 0 & 2 \\
\hline 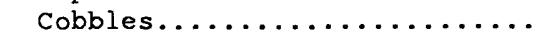 & 2 & 6 \\
\hline Fine sand with some coarse sand & 6 & -50 \\
\hline Fine to medium sand......... & 50 & -75 \\
\hline Refusal $\ldots \ldots \ldots \ldots \ldots \ldots$ & & at 75 \\
\hline TOWNSEND A1 & & \\
\hline Cuarse sand and gravel....... & 0 & -8 \\
\hline 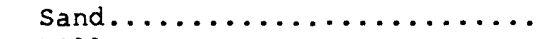 & 8 & -89 \\
\hline Till $\ldots \ldots \ldots \ldots \ldots \ldots \ldots \ldots$ & 89 & -100.5 \\
\hline Refusal $\ldots \ldots \ldots \ldots \ldots \ldots$ & & at 100.5 \\
\hline TOWNSEND A2 & & \\
\hline Medium to coarse sand and & & \\
\hline 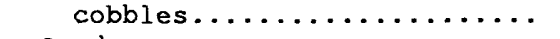 & 0 & -20 \\
\hline 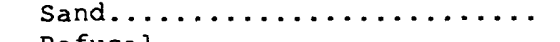 & 20 & -54 \\
\hline 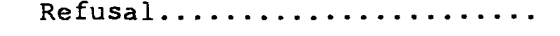 & & at 54 \\
\hline
\end{tabular}




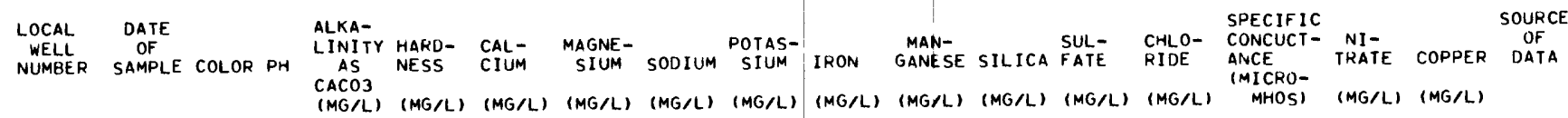

$\begin{array}{llrrrr}5 & 6- & -66 & 25 & 6.2 & 47\end{array}$

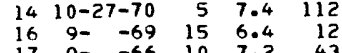
$\begin{array}{rrrrrr}17 & 0- & -66 & 10 & 7.2 & 43 \\ 17 & 12- & -66 & 2 & 7.2 & 44\end{array}$ $\begin{array}{rrrrrr}17 & 12- & -66 & 2 & 7.2 & 44 \\ 29 & 4- & -71 & 25 & 6.4 & 39\end{array}$ $\begin{array}{rrrrr}29 & 4- & -71 & 25 & 6.4 \\ 31 & 9- & -71 & 5 & 6.1\end{array}$ $\begin{array}{rrrrr}31 & 9- & -71 & 5 & 6.1 \\ 32 & 8- & -71 & 20 & 6.3\end{array}$ $33 \quad 7-\quad-71 \quad 3 \quad 6.3 \quad 12$ $34-10 \quad 7.1 \quad 28$ $\begin{array}{rrrrr}35 & 8-06-71 & 8 & 6.1 & 14 \\ 35 & 8-27-71 & 10 & 6.0 & 13\end{array}$

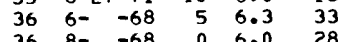
$\begin{array}{llllll}36 & 8- & -68 & 0 & 6.0 & 28 \\ 38 & 1- & -71 & 3 & 6.1 & 85\end{array}$ $\begin{array}{llrrrr}38 & 1- & -71 & 3 & 6.1 & 85 \\ 40 & 0-19-64 & 20 & 7.1 & 39\end{array}$ $4511-70$ - 00.2

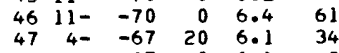
$\begin{array}{rrrrrr}47 & 4- & -67 & 20 & 6.1 & 34 \\ 48 & 11- & -67 & 0 & 6.1 & 9 \\ 55 & 0- & -71 & 15 & 6.1 & 16\end{array}$

\begin{tabular}{rrr}
64 & -- & - \\
\hline- & 90 & 90 \\
80 & -- & -- \\
2 & -- & - \\
40 & -- & - \\
12 & -- & - \\
12 & -- & - \\
20 & -- & - \\
-- & 36 & 36 \\
132 & 44 & - \\
146 & 50 & - \\
92 & -- & - \\
80 & -- & - \\
84 & -- & - \\
-- & 34 & 34 \\
54 & -- & - \\
46 & -- & - \\
104 & -- & - \\
74 & -- & - \\
34 & - & -
\end{tabular}

\section{ASHBURNHAM}

$\begin{array}{lllll}5 & 6-01-54 & 4 & 6.6 & 48\end{array}$ $\begin{array}{lllll}5 & 2-08-60 & 3 & 6.4 & 36 \\ 5 & 2-14-61 & 1 & 6.9 & 34 \\ 5-15-63 & 2 & 6.6 & 41\end{array}$ $\begin{array}{lllll}5 & 5-15-63 & 2 & 6.6 & 41 \\ 5 & 7-26-66 & 4 & 7.5 & 56\end{array}$

$\begin{array}{rr}71 & 24 \\ 66 & 2 \\ 93 & 30 \\ 72 & 2 \\ 61 & 20 \\ 166 & 5\end{array}$

70
12
16
--
34
34
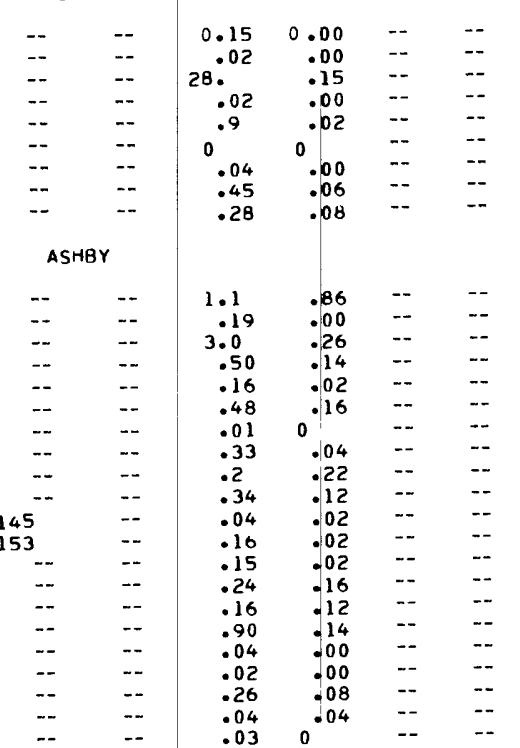

60.
3.
19.
4.5
2.5
7.5
10.5
1.5
5.0

$\begin{array}{cccr}-- & 1.0 & -- & 3 \\ -- & .05 & -- & 3 \\ -- & .30 & -- & - \\ -- & 1.7 & -- & 3 \\ -- & .00 & -- & -- \\ -- & .3 & -- & - \\ -- & 5.4 & -- & 3 \\ -- & .1 & - & - \\ & .0 & -- & 3\end{array}$

.28 .08 -.

$\begin{array}{llll}1.1 & .86 & -- & -- \\ .19 & .00 & -- & - \\ 3.0 & .26 & -- & - \\ .50 & .14 & -- & -- \\ .16 & .02 & -- & - \\ .48 & .16 & -- & -- \\ .01 & 0 & -- & - \\ .33 & .04 & -- & -- \\ .2 & .22 & -- & -- \\ .34 & .12 & -- & -- \\ .04 & .02 & -- & -- \\ .16 & .02 & -- & -- \\ .15 & .02 & -- & -- \\ .24 & .16 & -- & -- \\ .16 & .12 & -- & -- \\ .90 & .14 & -- & -- \\ .04 & .00 & -- & -- \\ .02 & .00 & -- & -- \\ .26 & .08 & -- & -- \\ .04 & .04 & -- & -- \\ .03 & 0 & -- & --\end{array}$

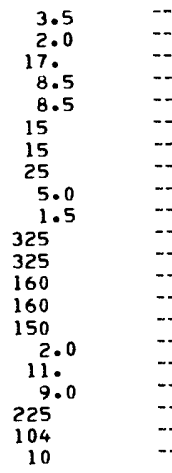

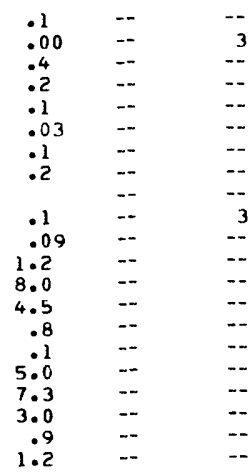

$\begin{array}{rlrrrr}59 & 0- & -69 & 0 & 8.7 & 53 \\ 60 & 2-13-56 & 0 & 6.9 & -2 \\ 62 & 4-21-60 & & 8.3 & 58 \\ 114 & 4- & -70 & 50 & 6.3 & 30 \\ w & 115 & 2-13-61 & 6 & 7.2 & 27 \\ 117 & 2-13-61 & 7 & 7.1 & 29 \\ w & 117 & 3-10-61 & 4 & 7.0 & 27 \\ 121 & 3-13-61 & 3 & 6.8 & 23\end{array}$

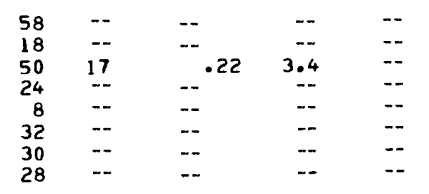

$: 03$
$: 00$
$\vdots$
$\vdots$

$\begin{array}{lll}.00 & & \\ .09 & .18 & 14 \\ .02 & .1 & 14 \\ .00 & .32 & 13 \\ .03 & .60 & 12\end{array}$

$4.1 \quad 14$

AYER

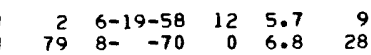

BOYLSTON

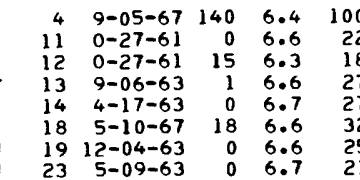

$\begin{array}{lll}27 & -- & - \\ 40 & -- & -\end{array}$

\begin{tabular}{lc}
-- & -- \\
-- & -- \\
\multicolumn{2}{c}{ CLINTON } \\
-- & -- \\
-- & -- \\
-- & -- \\
-- & -- \\
-- & -- \\
-- & - \\
-- & -
\end{tabular}

.80
.05

13

--
--
--
18
83

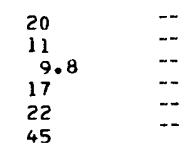

$\begin{array}{rr}2.2 & -- \\ 5.0 & -- \\ 7.7 & -- \\ 5.1 & -- \\ .9 & -- \\ 5.3 & -\end{array}$

$\begin{array}{llll}.03 & .04 & - & -- \\ .06 & .0 & -- & -- \\ .06 & - & 16 & 3.3 \\ .0 & .50 & -- & -. \\ .13 & .00 & -- & -- \\ .18 & 000 & -- & -- \\ .04 & .00 & -- & - \\ .03 & .00 & -- & --\end{array}$

2.0
3.0
4.7
2.0
1.0
2.0
2.0
1.0

$\begin{array}{cccc}-- & .0 & -- & - \\ -- & 1.5 & -- & - \\ -- & .03 & -- & -- \\ -- & .0 & -- & - \\ -- & .05 & -- & 3 \\ - & .05 & -- & 3 \\ -- & .05 & -- & 3 \\ & .30 & -- & 3\end{array}$

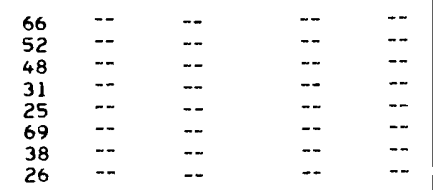

$\begin{array}{rccc}11 & 2.4 & -- & -- \\ .03 & 00 & -- & - \\ .55 & .06 & -- & - \\ .02 & .00 & -- & -- \\ .07 & 000 & -- & -- \\ .65 & 005 & -- & -- \\ .02 & 000 & -- & -- \\ .00 & .00 & -- & --\end{array}$

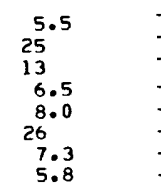

$\begin{array}{cccc}- & .10 & - & - \\ - & 1.0 & -- & 3 \\ - & .06 & -- & 3 \\ -- & 2.1 & -- & - \\ -- & .26 & -- & - \\ -- & -- & -- & - \\ -- & 2.1 & -- & - \\ - & .70 & -- & -\end{array}$
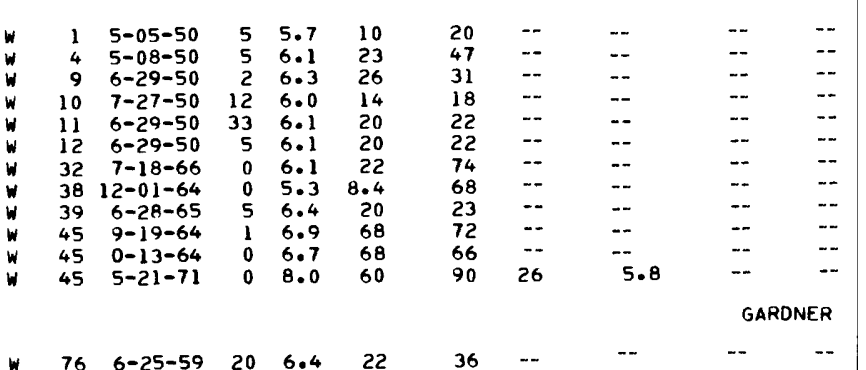

$\begin{array}{llll}.09 & .00 & -- & -- \\ .03 & .00 & -- & -- \\ .03 & .20 & -- & -- \\ .75 & .06 & -- & -- \\ 1.4 & .35 & -- & -- \\ .61 & .50 & -- & -- \\ .12 & .00 & -- & -- \\ .28 & .08 & -- & -- \\ .42 & .0 & -- & -- \\ .23 & .55 & -- & -- \\ .09 & .45 & -- & --\end{array}$

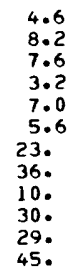

4.6
8.2
7.6
3.2
7.0
5.6
23.
36.
10.
30.
29.
45.

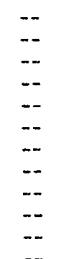

$\begin{array}{lll}.34 & -- & 3 \\ 3.0 & -- & 3 \\ .2 & -- & 3 \\ .10 & -- & 3 \\ .15 & -- & 3 \\ .12 & -- & 3 \\ 2.4 & -- & -- \\ 1.1 & -- & -- \\ .12 & -- & -- \\ .08 & -- & -- \\ .18 & -- & - \\ .10 & 0 & 3\end{array}$

$\begin{array}{lllll}76 & 6-25-59 & 20 & 6.4 & 22\end{array}$ 
Table 3.--Chemical analyses of ground water (Continued)

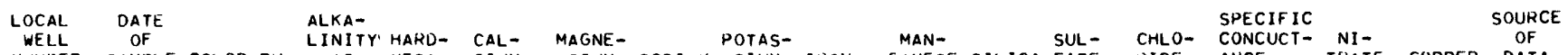
NUMBEP SAMPLE COLOR PH AS NESS CIUM SIUM SODIUM SIUM IRON GANESE SILICA FATE RIDE ANCE TRATE CUPPER DATA

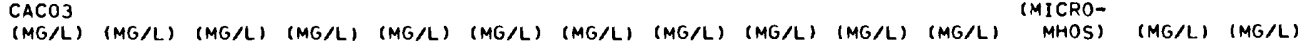

GROTON

$\begin{array}{rr}94 & -- \\ & \\ 26 & -- \\ 2 & -- \\ 56 & -- \\ 72 & -- \\ 82 & 28 \\ 84 & 78 \\ -- & -- \\ 92 & 31 \\ 93 & 32 \\ 48 & -- \\ 70 & -- \\ 46 & -- \\ 0 & -- \\ 124 & -- \\ 102 & 32 \\ 96 & 31 \\ 96 & 30 \\ 94 & 30 \\ 110 & 33 \\ 140 & 46\end{array}$

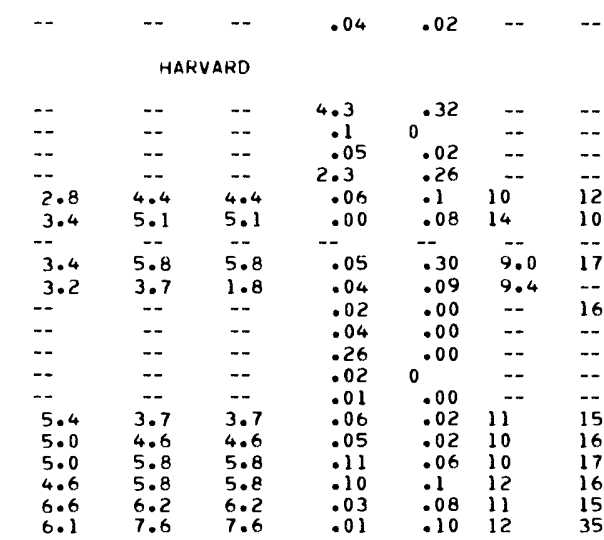

10.

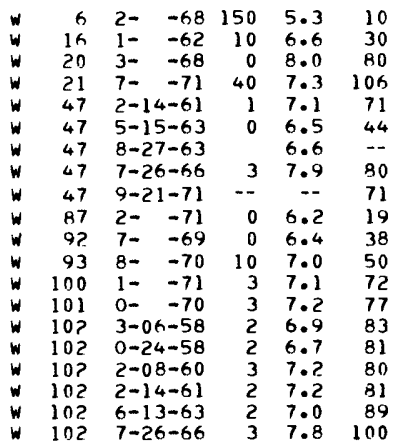

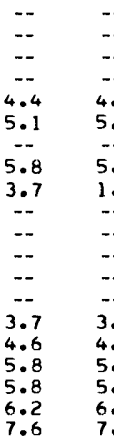

HOLDEN
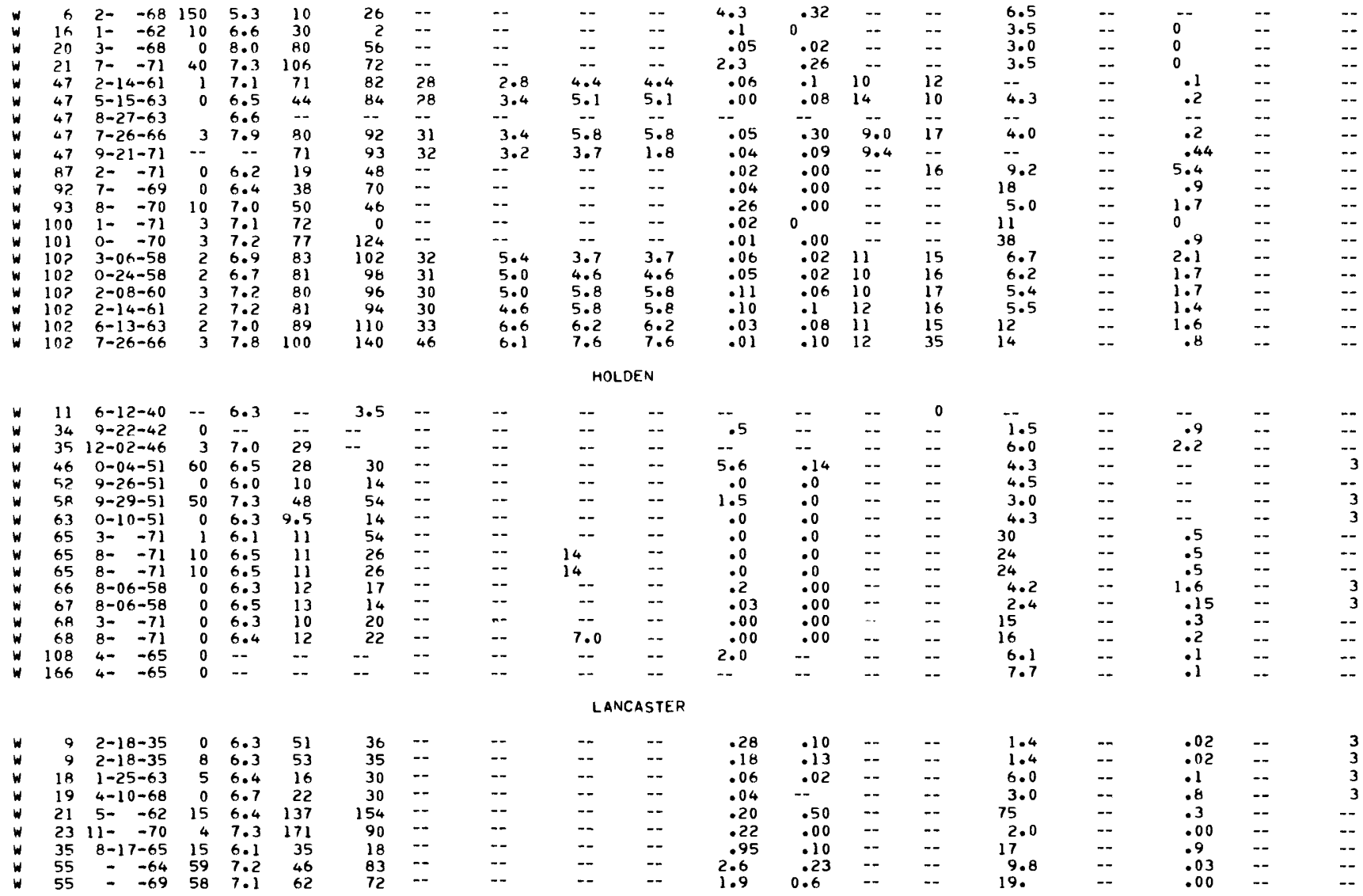

LANCASTER
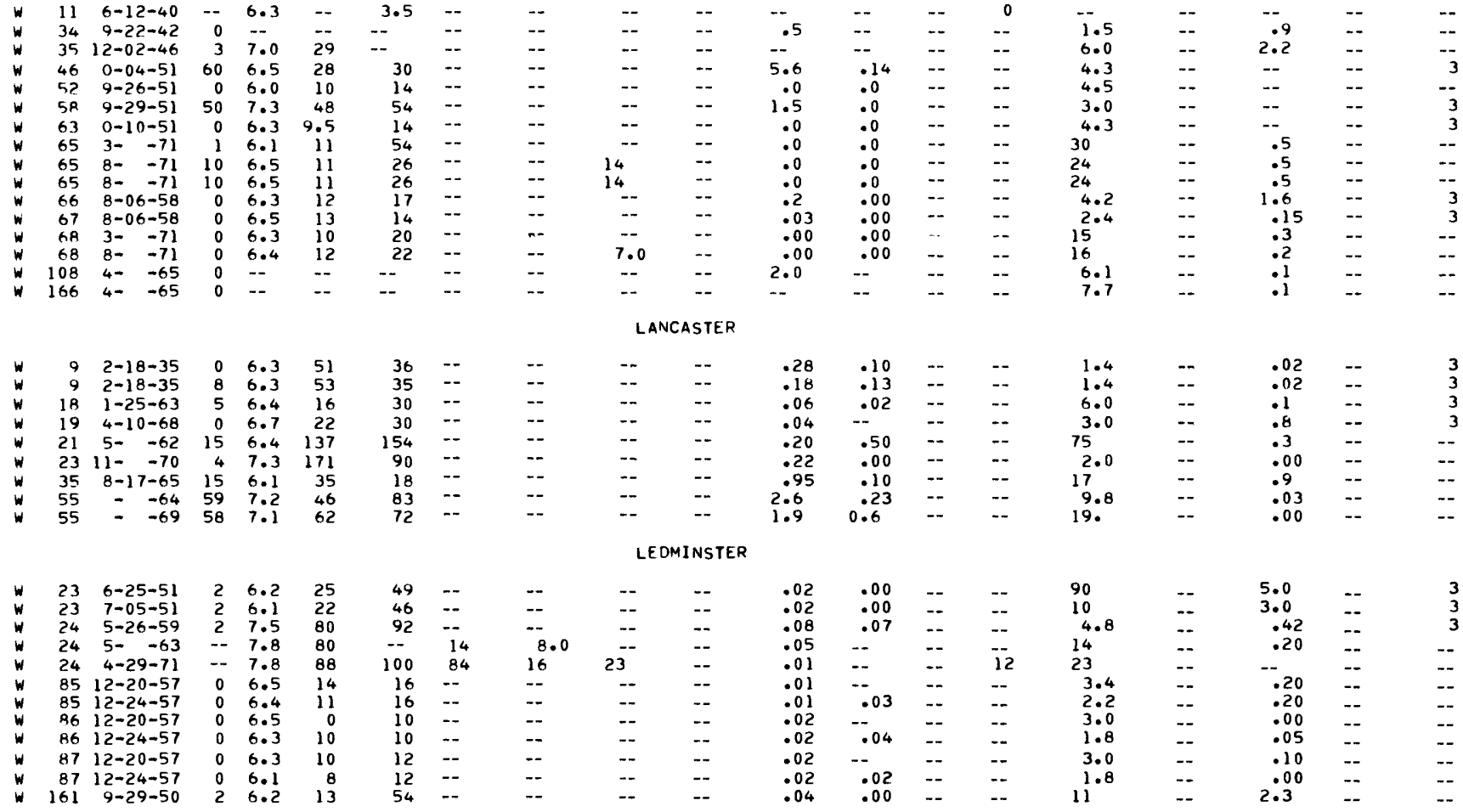

LEDMINSTER
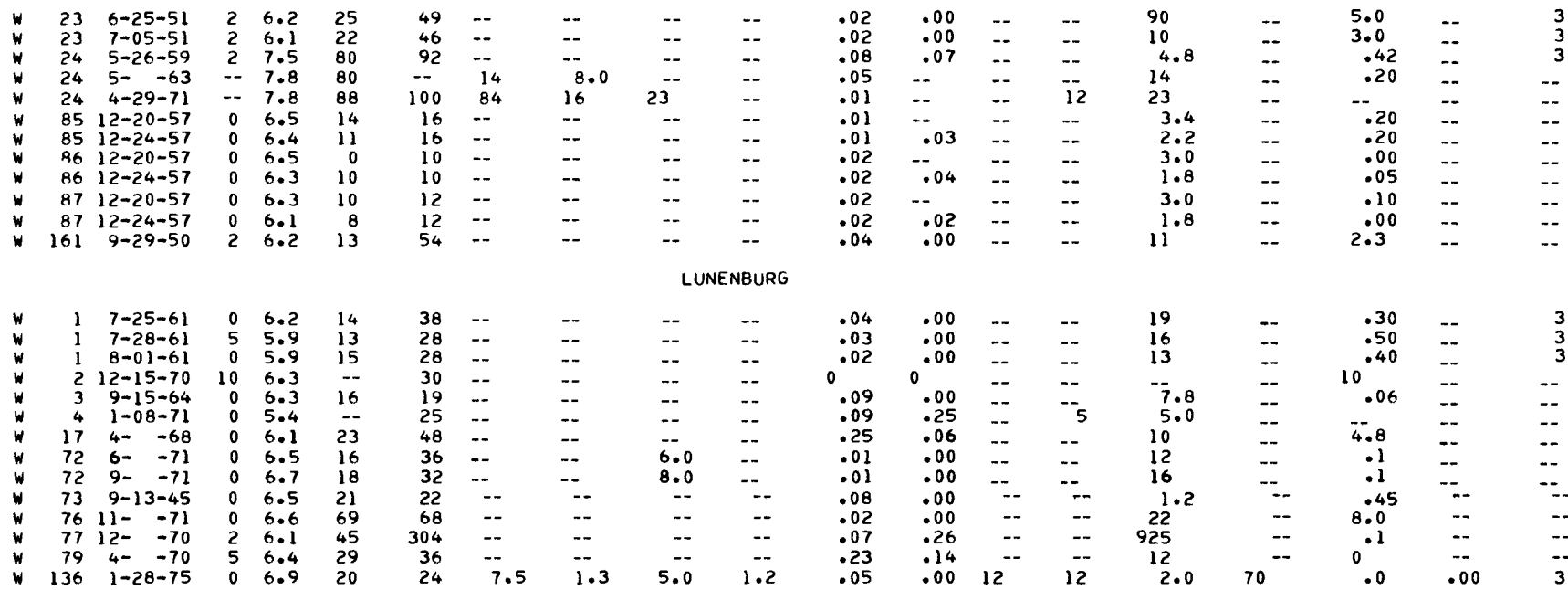

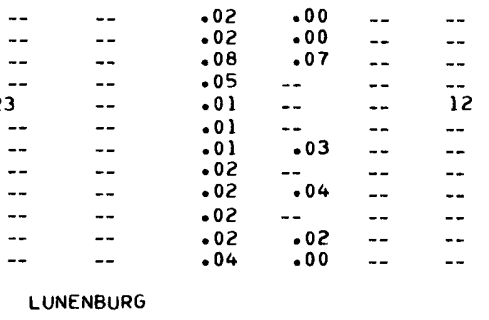

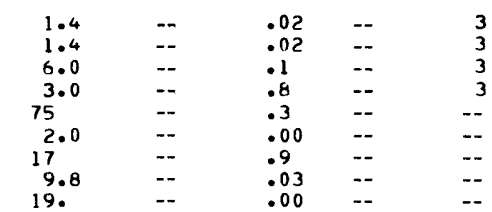

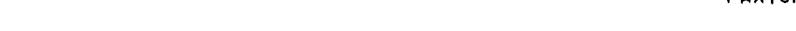

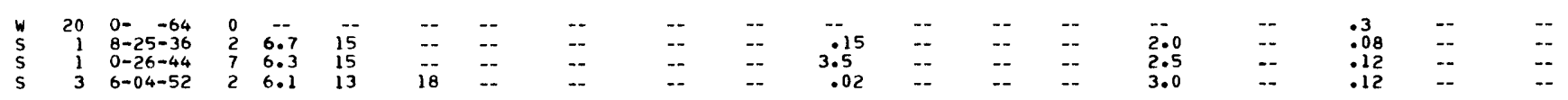


Table 3.--Chemical analyses of ground water (Continued)

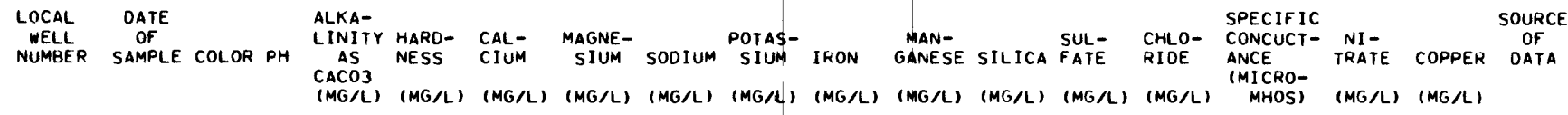
PEPPERELL

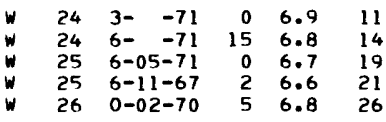

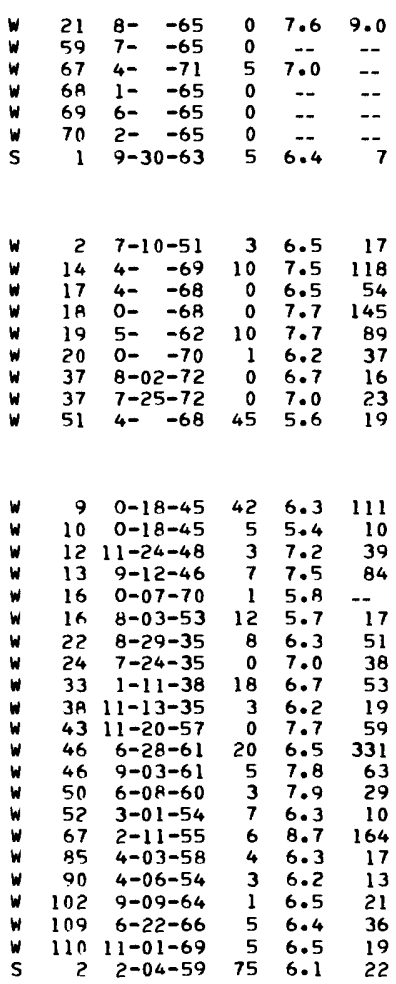

$\begin{array}{lllrr}20 & - & -- & 4.0 & -- \\ 20 & -- & -- & 4.0 & -- \\ 52 & -- & -- & -- & -- \\ 60 & -- & -- & -- & -- \\ 44 & -- & -- & - & \end{array}$

PRINCETON

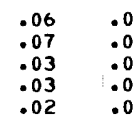

$\begin{array}{llll}-- & -- & -- & - \\ -- & - & -- & - \\ .00 & .04 & -- & - \\ -- & -- & -- & - \\ -- & -- & -- & - \\ -- & -- & -- & -- \\ .03 & .02 & -- & -\end{array}$
7.6
20
46
26
22
5.0

5.8
10
14
14
1.0
14
2.0
6.0
27

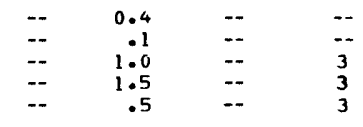

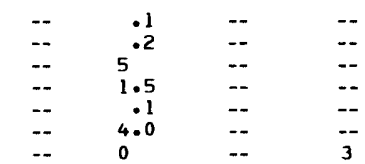
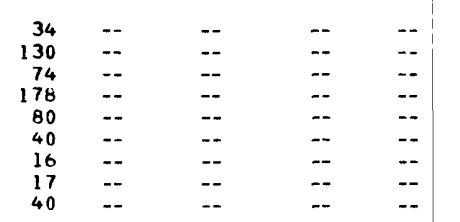

\begin{tabular}{l|lll}
.09 & .00 & -- & -- \\
.17 & .02 & -- & -- \\
.07 & .10 & -- & -- \\
.01 & .04 & -- & -- \\
.07 & .00 & -- & -- \\
.01 & .00 & -- & -- \\
.02 & .01 & -- & -- \\
.01 & .00 & -- & -- \\
.14 & .06 & -- & --
\end{tabular}

STERLING

\begin{tabular}{|c|c|}
\hline -- & -- \\
\hline Tr & - \\
\hline -- & -- \\
\hline 114 & -- \\
\hline 58 & -- \\
\hline 43 & - \\
\hline 51 & $\rightarrow$ \\
\hline-- & -- \\
\hline-- & -- \\
\hline 52 & - \\
\hline-- & - \\
\hline 58 & -- \\
\hline 52 & \\
\hline 46 & -- \\
\hline $\begin{array}{l}12 \\
43\end{array}$ & $\overline{--}$ \\
\hline $\begin{array}{l}43 \\
22\end{array}$ & -- \\
\hline 38 & -- \\
\hline 60 & -- \\
\hline 12 & $\cdots$ \\
\hline
\end{tabular}

19

\begin{tabular}{|c|c|c|c|}
\hline & -- & - & $\cdots$ \\
\hline .05 & -- & -- & - \\
\hline .25 & -- & - & - \\
\hline .05 & - & -- & \\
\hline $\begin{array}{l}.07 \\
1.08\end{array}$ & .68 & $=$ & \\
\hline 1.1 & .01 & -- & \\
\hline .12 & .00 & -- & \\
\hline 1.4 & -- & -- & \\
\hline $\begin{array}{r}.40 \\
.03\end{array}$ & $\because$ & -- & \\
\hline .60 & .00 & -- & \\
\hline .05 & .00 & - & \\
\hline .18 & .00 & $\begin{array}{l}-- \\
--\end{array}$ & \\
\hline .13 & - & -- & \\
\hline .16 & .00 & -- & \\
\hline .18 & -- & -- & \\
\hline .05 & .02 & -- & \\
\hline $\begin{array}{l}.02 \\
.04\end{array}$ & $\begin{array}{l}.02 \\
.00\end{array}$ & -. & \\
\hline & & & \\
\hline
\end{tabular}

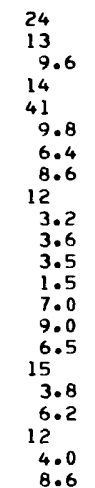

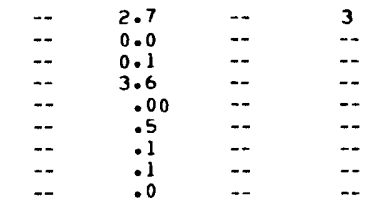
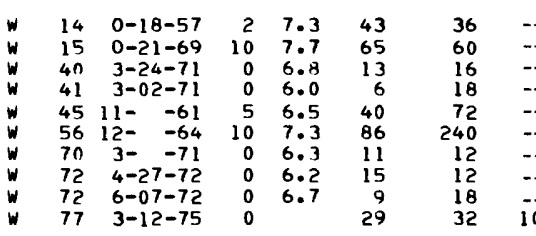

TOWNSENO

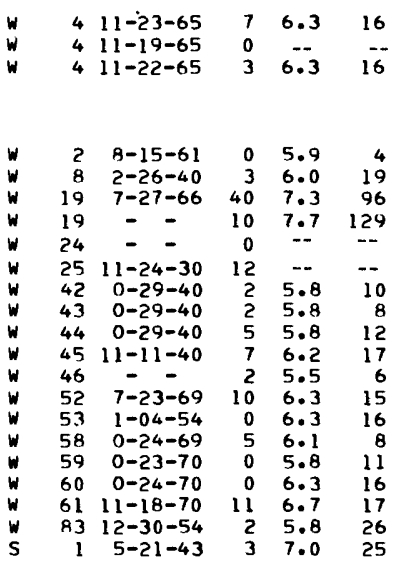

$\begin{array}{lll}17 & -- & - \\ 42 & -- & -- \\ 17 & -- & --\end{array}$

\section{WEST BOYLTION}

\begin{tabular}{l|lll}
.15 & .00 & -- & -- \\
.03 & .02 & -- & -- \\
.03 & .00 & -- & -- \\
.10 & .04 & -- & -- \\
.04 & .02 & -- & -- \\
.02 & .02 & -- & -- \\
.00 & .00 & -- & -- \\
.01 & .00 & -- & -- \\
.01 & .00 & -- & -- \\
.05 & .01 & 9.8 & 5
\end{tabular}

$\begin{array}{cc}1.6 & - \\ 3.5 & - \\ 2.0 & - \\ 7.0 & - \\ 30 & - \\ 20 & = \\ 4.5 & - \\ 2.5 & = \\ 2.0 & = \\ 27 & 100\end{array}$

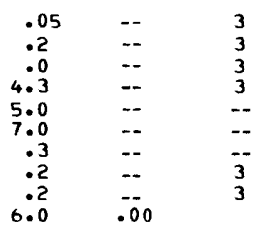

$\begin{array}{lllll}9.0 & -- & .04 & -- & -- \\ 8.0 & -- & .2 & -- & -- \\ 9.0 & -- & .4 & -- & -\end{array}$

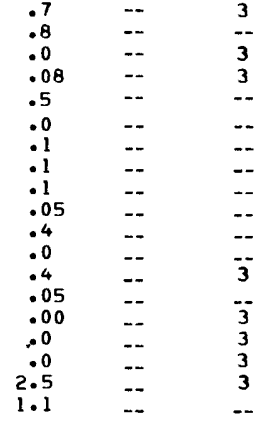


Table 4.--Chemical analys is of precipitation, October 29, 1973

(Analytical results in milligrams per liter except as indicated. Analyses by U.S. Geological Survey.)

Ashby, Massachusetts, Lat 42040'24", long 71046'33"

Middlesex County, elev. $488 \mathrm{ft}$. At weather station at Willard Brook State Forest headquarters.

\begin{tabular}{|c|c|}
\hline Calcium (Ca) & 0 \\
\hline Magnes ium (Mg) & .10 \\
\hline Sodium (Nà) & .50 \\
\hline Potassium $(K)$ & .10 \\
\hline Bicarbonate $\left(\mathrm{HCO}_{3}\right)$ & 2.00 \\
\hline Carbonate $\left(\mathrm{CO}_{3}\right)$ & 0 \\
\hline Sulfate $\left(\mathrm{SO}_{4}\right)$ & 1.00 \\
\hline Chloride $(\mathrm{Cl})$ & 1.20 \\
\hline Fluoride & .10 \\
\hline Nitrate $\left(\mathrm{NO}_{3}\right)$ & .087 \\
\hline Nitrite $\left(\mathrm{NO}_{2}\right)$ & .003 \\
\hline Alkalinity, total as $\mathrm{CaCO}_{3}$ & 2.000 \\
\hline $\begin{array}{l}\text { Specific conductance } \\
\text { (micromhos) }\end{array}$ & 14 \\
\hline Tota1 hardness & 0 \\
\hline Silica & 0 \\
\hline $\mathrm{pH}$ & 4.7 \\
\hline
\end{tabular}


Table 5.--Water levels in observation wells

[Water levels in feet below land-surface datum. Measured by U.S. Geological Survey.]

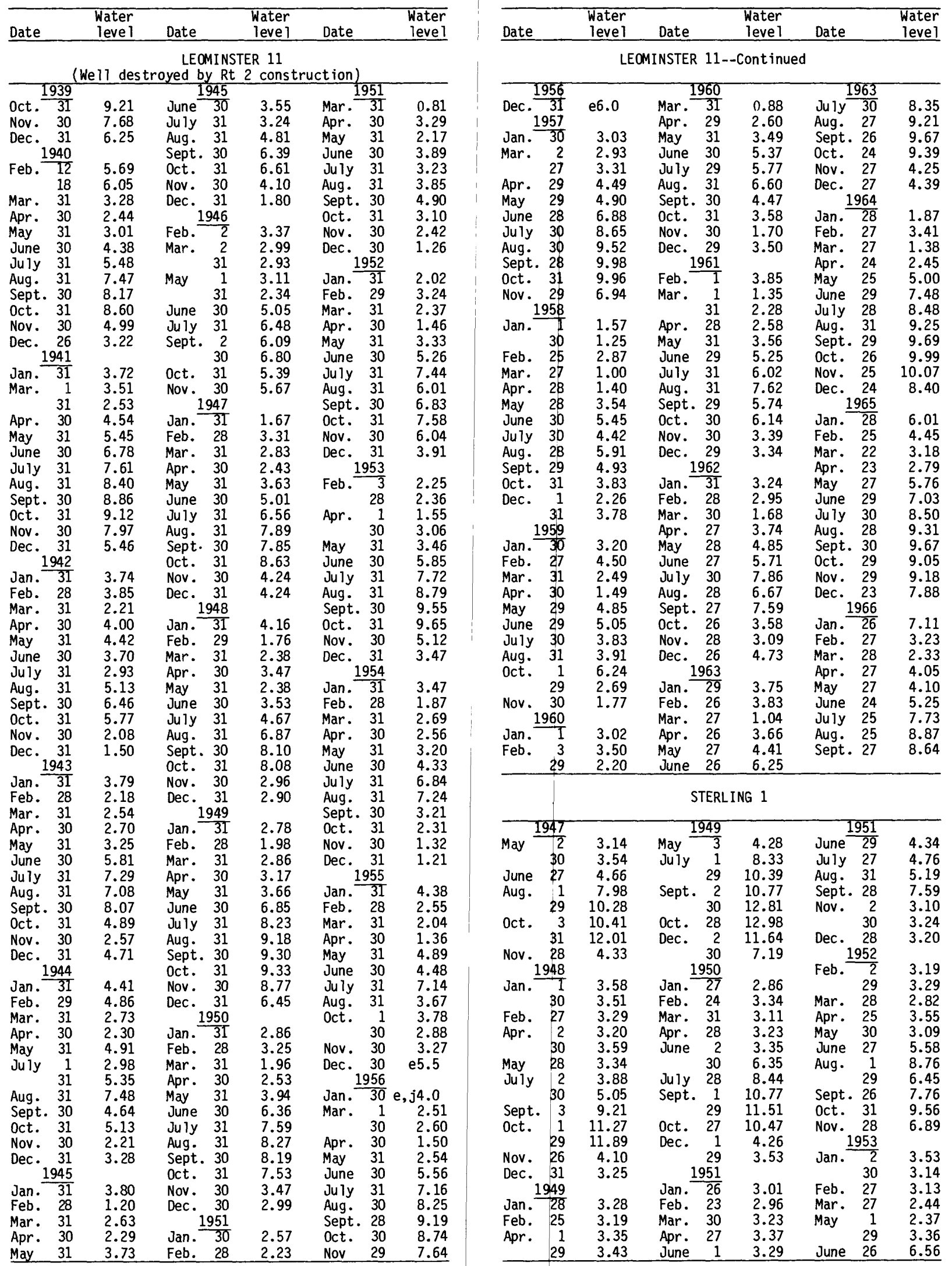

e estimated.

j frozen. 
Table 5.--Water levels in observation wells--Cont inued

\begin{tabular}{lllll}
\hline Wate & $\begin{array}{l}\text { Water } \\
\text { level }\end{array}$ & Date & $\begin{array}{l}\text { Water } \\
\text { level }\end{array}$ Date & Water \\
leve
\end{tabular}

STERLING 1--Continued

\begin{tabular}{|c|c|c|c|c|c|}
\hline$\frac{1953}{31}$ & 9.96 & $\frac{1959}{31}$ & 4.26 & $\frac{1965}{28}$ & 5.27 \\
\hline 1954 & & $\begin{array}{l}31 \\
\text { fug. } 28\end{array}$ & 4.20 & $\begin{array}{ll}\text { Jan. } & 28 \\
\text { Feb. } & 26\end{array}$ & $\begin{array}{l}3.21 \\
3.70\end{array}$ \\
\hline in. 27 & 3. & $c \hat{t}$. & & Mar. 22 & 3. \\
\hline $\begin{array}{l}\text { eb. } 26 \\
\text { ar. }\end{array}$ & & 30 & & pr. 23 & 3 \\
\hline $\begin{array}{ll}\text { ar. } & 26 \\
\text { pr. } & 30\end{array}$ & $\begin{array}{l}3.01 \\
3.25\end{array}$ & Nov. $\begin{array}{r}27 \\
1960 \\
\end{array}$ & & $\begin{array}{ll}\text { May } & 27 \\
\text { June } & 28\end{array}$ & $\begin{array}{l}4.57 \\
6.73\end{array}$ \\
\hline ay 28 & & Jan. 1 & 3.17 & July 30 & 10. \\
\hline $\begin{array}{ll}\text { une } & 25 \\
\text { uly } & 30\end{array}$ & & Feb. & & $\begin{array}{ll}\text { Aug. } & 24 \\
\text { Sept. } & 30\end{array}$ & \\
\hline dg. 27 & 7 & Apr. $\quad 1$ & 2.46 & 0ct. 29 & 14. \\
\hline$c t$. & & 29 & & Nov. 29 & \\
\hline ov. & & $\begin{array}{lr}\text { May } & 27 \\
\text { July } & 1\end{array}$ & $\begin{array}{l}3.02 \\
5.86\end{array}$ & Dec. $\begin{array}{r}23 \\
1966\end{array}$ & \\
\hline c. 31 & & 29 & & Jan. -26 & 7.48 \\
\hline 1955 & & Sept. & & $\begin{array}{ll}\text { Feb. } & 27 \\
\text { Mar. }\end{array}$ & 3. \\
\hline b. 25 & & ct. 28 & & Apr. 27 & \\
\hline pr. & $\begin{array}{l}3 . \\
2 .\end{array}$ & $\begin{array}{r}2 \\
30\end{array}$ & $\begin{array}{l}3 . \\
2 .\end{array}$ & $\begin{array}{ll}\text { May } & 27 \\
\text { June } & 24\end{array}$ & 3. \\
\hline 27 & 4. & 1961 & & July 25 & 8. \\
\hline ine & & $\operatorname{lan} . \frac{27}{27}$ & 3.26 & Aug. & 11 \\
\hline uly 27 & & Mar. & & Sept. 26 & \\
\hline $\begin{array}{ll}\text { ug. } & 26 \\
\text { ept. } & 30\end{array}$ & & $\begin{array}{l}31 \\
28\end{array}$ & & $\begin{array}{ll}\text { Oct. } & 27 \\
\text { Nov. } & 26\end{array}$ & 4 \\
\hline ct. 28 & & 29 & 3. & Dec. 29 & \\
\hline ec. & & $\begin{array}{ll}\text { une } & 30 \\
\text { uly } & 28\end{array}$ & & $\frac{1967}{27}$ & \\
\hline 1956 & & dg. 28 & & Feb. 28 & 3. \\
\hline $\mathrm{b}$. & 3. & pt. 29 & 7 & Mar. 30 & 2. \\
\hline & & t. 28 & & Apr. 28 & $\begin{array}{l}2 \\
3\end{array}$ \\
\hline $\begin{array}{ll}\text { ar. } & 30 \\
\text { r. } & 27\end{array}$ & 3. & $\begin{array}{ll}\text { Nov. } & 28 \\
\text { Dec. } & 29\end{array}$ & 3. & $\begin{array}{ll}\text { May } & 23 \\
\text { June } & 28\end{array}$ & 3 \\
\hline une & 3. & 1962 & & Julv & \\
\hline & 5. & n. 22 & & Aug. 25 & \\
\hline Aly & 8. & b. 27 & & Sept. 29 & \\
\hline dg. 31 & 11. & ir. $\quad 28$ & & Oct. 25 & \\
\hline ov. 2 & 11. & y. 27 & 2 & Dec. 26 & \\
\hline & & ine 27 & & $\underline{1968}$ & \\
\hline $\begin{array}{l}28 \\
1957\end{array}$ & & $\begin{array}{ll}17 y & 27 \\
19 . & 27\end{array}$ & & $\begin{array}{ll}\text { Jan. } & 29 \\
\text { Feb. } & 23\end{array}$ & \\
\hline b. & 3. & pt. 28 & 10 & Mar. 27 & \\
\hline ar. & & 27 & & Apr. 23 & \\
\hline r. & 3. & $\begin{array}{ll}\text { ov. } & 27 \\
\text { ec. } & 28\end{array}$ & 3. & $\begin{array}{l}\text { May } \\
\text { June }\end{array}$ & \\
\hline & & 196 & & 71 & \\
\hline une & 5. & 1. 28 & 3. & Aug. & \\
\hline dg. & 10 & 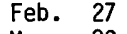 & & Sept. 25 & \\
\hline & & $\therefore$ & & $\begin{array}{ll}\text { Oct. } 25 \\
\end{array}$ & \\
\hline 25 & & $\because \quad 29$ & & $\begin{array}{ll}\text { Nov. } & 22 \\
\text { Dec. } & 27\end{array}$ & \\
\hline v. 29 & & 28 & & 1969 & \\
\hline c. & & & & $\operatorname{Jan} . \overline{24}$ & \\
\hline 1 & & & & Feb. 2 & \\
\hline in. & 3. & t. 27 & & Mar. & \\
\hline & & & & Apr. & \\
\hline & & & & & \\
\hline r. & $\begin{array}{l}3 \\
3\end{array}$ & $\begin{array}{l}\text { Dec. } 27 \\
1964\end{array}$ & & $\begin{array}{l}\text { June } \\
\text { July }\end{array}$ & \\
\hline & 4. & 0 & & $\begin{array}{l}\text { Ju } \\
\text { Au }\end{array}$ & \\
\hline & & & & Sept. & \\
\hline & & $=$ & & $0 \mathrm{ct}$. & \\
\hline & & 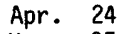 & & Nov. & \\
\hline & & & & Dec. 24 & 2. \\
\hline v. $\begin{array}{r}28 \\
1959\end{array}$ & & 28 & 7 & $\frac{1970}{23}$ & \\
\hline & 3.2 & . 31 & & . 20 & \\
\hline & 2.87 & Sept. 29 & 13. & Mar. 25 & \\
\hline 27 & 3.3 & 26 & 14.60 & Apr. & \\
\hline & 2.8 & v. & & May & \\
\hline & & Dec. 28 & 13.16 & July & \\
\hline & & & & & \\
\hline
\end{tabular}

\begin{tabular}{|c|c|c|c|c|c|}
\hline ate & $\begin{array}{l}\text { Water } \\
\text { level }\end{array}$ & ate & $\begin{array}{l}\text { Water } \\
\text { level }\end{array}$ & Date & $\begin{array}{l}\text { Water } \\
\text { leve } 1\end{array}$ \\
\hline \multicolumn{6}{|c|}{ STERLING 1--Continued } \\
\hline $\begin{array}{lr} & \frac{1970}{19} \\
\text { Sept. } & 23 \\
\text { Oct. } & 26 \\
\text { Nov. } & 23 \\
\text { Dec. } & 24 \\
& \frac{1971}{25} \\
\text { Jan. } & \frac{1}{25} \\
\text { Feb. } & 23 \\
\text { Mar. } & 24 \\
\text { Apr. } & 26 \\
\text { May } & 21 \\
\text { June } & 28 \\
\text { July } & 26 \\
\text { Aug. } & 25 \\
\text { Sept. } & 22 \\
\text { Oct. } & 27 \\
\text { Nov. } & 24 \\
\text { Dec. } & 23 \\
& 1972 \\
\text { Jan. } & 26 \\
\end{array}$ & $\begin{array}{r}11.80 \\
12.60 \\
9.97 \\
6.27 \\
\\
4.91 \\
2.70 \\
2.79 \\
3.04 \\
3.01 \\
5.84 \\
9.44 \\
11.19 \\
11.32 \\
11.18 \\
9.87 \\
2.96\end{array}$ & $\begin{array}{ll}\text { Feb. } & \frac{1972}{28} \\
\text { Mar. } & 27 \\
\text { Apr. } & 25 \\
\text { May } & 24 \\
\text { June } & 28 \\
\text { July } & 26 \\
\text { Aug. } & 28 \\
\text { Sept. } & 26 \\
\text { Oct. } & 30 \\
\text { Nov. } & 27 \\
\text { Dec. } & 22 \\
& \frac{1973}{26} \\
\text { Jan. } & \frac{1}{26} \\
\text { Feb. } & 23 \\
\text { Mar. } & 26 \\
\text { Apr. } & 23 \\
\text { May } & 24 \\
\text { June } & 26 \\
\text { July } & 27\end{array}$ & $\begin{array}{r}2.94 \\
2.77 \\
2.78 \\
2.90 \\
3.20 \\
4.58 \\
8.48 \\
10.35 \\
8.88 \\
2.75 \\
2.77\end{array}$ & $\begin{array}{lr} & \frac{1973}{27} \\
\text { Aug. } & \frac{17}{\text { Sept. }} 25 \\
\text { Oct. } 26 \\
\text { Nov. } & 27 \\
\text { Dec. } & 21 \\
& \frac{1974}{24} \\
\text { Jan. } & 25 \\
\text { Feb. } & 25 \\
\text { Mar. } & 26 \\
\text { Apr. } & 29 \\
\text { May } & 22 \\
\text { June } & 27 \\
\text { July } & 26 \\
\text { Aug. } & 28 \\
\text { Sept. } & 27 \\
\text { Oct. } & 31\end{array}$ & $\begin{array}{r}8.75 \\
10.75 \\
12.14 \\
9.66 \\
3.00 \\
\\
2.95 \\
3.00 \\
3.02 \\
3.08 \\
3.23 \\
3.29 \\
7.10 \\
1.80 \\
10.35 \\
5.66\end{array}$ \\
\hline
\end{tabular}

TOWNSEND 13

\begin{tabular}{|c|c|c|c|c|c|}
\hline 1965 & & 1968 & & 1971 & \\
\hline n. & .75 & May & 12.46 & ept. 24 & 14.33 \\
\hline b. 25 & & June 26 & & ct. 27 & \\
\hline ar. 23 & 1 & July 26 & & lov. 29 & 4.98 \\
\hline pr. 29 & 1 & Aug. 27 & & Dec. 23 & 14.78 \\
\hline & & Sept. 25 & & $\frac{1972}{20}$ & \\
\hline 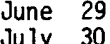 & & ct. 29 & & an. 28 & $\begin{array}{l}14.36 \\
14.04\end{array}$ \\
\hline ug. 28 & & Dec. 26 & & Mar. 29 & 13.18 \\
\hline ept. 30 & & 1969 & & Apr. & 11.63 \\
\hline t. 29 & 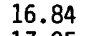 & Jan. $\overline{28}$ & 13 & May & 10.77 \\
\hline ov. 29 & 17. & Feb. & & June 28 & 11.62 \\
\hline Dec. 23 & & Mar. & & July 28 & 13 \\
\hline an. $\frac{1700}{26}$ & 17. & May & 11 & Sept. 28 & 20 \\
\hline 27 & & June & 12 & 0ct. 27 & 1 \\
\hline 24 & 16. & July & & Nov. & 13 \\
\hline $\begin{array}{ll}r . & 27 \\
\text { iy } & 25\end{array}$ & $\begin{array}{l}15 . \\
15 .\end{array}$ & $\begin{array}{l}\text { Aug. } 27 \\
\text { Sept. } 24\end{array}$ & & Dec. $\begin{array}{r}28 \\
1973\end{array}$ & 12.8 \\
\hline une 24 & & oct. 29 & 14 & $\operatorname{Jan} . \quad 30$ & 12 \\
\hline uly & & Nov. & & Feb. $\quad 27$ & \\
\hline ug. & 15 & Dec. $\quad 30$ & & Mar. & 10.97 \\
\hline 28 & & 19 & & Apr. & 10 \\
\hline vi. & & =eb. & & $\begin{array}{l}\text { May } \\
\text { June }\end{array}$ & \\
\hline c. & & Mar. & 11 & Juły & 11 \\
\hline 196 & & $A p r$ & & Aug. & \\
\hline n. 27 & 15. & May & 11 & Sept. 27 & 13 \\
\hline 28 & & June & & Oct. & \\
\hline 29 & 15 & July & & Nov. & 14 \\
\hline $\begin{array}{ll}r . & 28 \\
y & 26\end{array}$ & $\begin{array}{l}13 . \\
12 .\end{array}$ & $\begin{array}{l}\text { Aug. } 27 \\
\text { Sept. } 25\end{array}$ & & Dec. $\begin{array}{r}28 \\
1974\end{array}$ & \\
\hline 27 & & Oct. & & n. 2 & \\
\hline uly & & Nov. & & Feb. & \\
\hline 30 & & Dec. 2 & & Mar. & 12.45 \\
\hline ept. & & 37 & & & \\
\hline 2 & & Feb. & 15 & June & \\
\hline 2 & & Mar. & 14 & $\mathrm{Ju}$ & \\
\hline 1968 & & Apr. & & & \\
\hline an. 26 & 14. & & 11 & Sept. 2 & \\
\hline 07 & 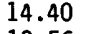 & June & 12 & 0 ct. 31 & 14.38 \\
\hline $\begin{array}{l}\text { r. } \quad 27 \\
\text { r. } \quad 25\end{array}$ & & $\begin{array}{l}\text { July } \\
\text { Aug. }\end{array}$ & $\begin{array}{l}13 \\
13\end{array}$ & & \\
\hline
\end{tabular}


Table 6.--Stream sites and discharge measurements

\begin{tabular}{|c|c|c|c|c|c|}
\hline $\begin{array}{l}\text { Map } \\
\text { no. }\end{array}$ & $\begin{array}{l}\text { Stream and } \\
\text { U.S. Geolog- } \\
\text { ical Survey } \\
\text { station no. }\end{array}$ & Location & $\begin{array}{l}\text { Drainage } \\
\text { area } \\
\left(m i^{2}\right)\end{array}$ & Date & $\begin{array}{c}\text { Discharge } \\
\left(\mathrm{ft}^{3} / \mathrm{s}\right)\end{array}$ \\
\hline 1 & $\begin{array}{l}01094340 \\
\text { Whitman } \\
\text { River }\end{array}$ & $\begin{array}{l}\text { Lat } 42033^{\prime} 35^{\prime \prime}, \text { long } 71052 \cdot 02^{\prime \prime}, \\
\text { Worcester County, at State } \\
\text { Highway } 2 \mathrm{~A}, 2.5 \text { miles nor theast } \\
\text { of Westminster, Mass. }\end{array}$ & -- & $\begin{array}{l}3-19-73 \\
7-30-73 \\
8-27-73 \\
8-29-73 \\
1-08-74\end{array}$ & $\begin{array}{l}173 \\
7.9 \\
12 \\
11 \\
40\end{array}$ \\
\hline 2 & $\begin{array}{l}01094342 \\
\text { Whitman } \\
\text { River } \\
\text { tributary }\end{array}$ & $\begin{array}{l}\text { Lat } 42^{\circ} 33^{\prime} 24^{\prime \prime}, \text { long } 71^{\circ} 52^{\prime} 25^{\prime \prime} \text {, } \\
\text { Worcester County, at Depot Rd., } \\
2 \text { miles northeast of Westminster, } \\
\text { Mass. }\end{array}$ & - & $\begin{array}{l}7-30-73 \\
8-27-73 \\
8-29-73\end{array}$ & $\begin{array}{l}3.1 \\
1.3 \\
1.6\end{array}$ \\
\hline 3 & $\begin{array}{l}01094350 \\
\text { Flag Brook }\end{array}$ & $\begin{array}{l}\text { Lat } 42032^{\prime} 07^{\prime \prime} \text {, long } 71051^{\prime} 01^{\prime \prime} \text {, } \\
\text { Worcester County, at access road } \\
\text { to Fitchburg mun ic ipal dump, } \\
0.5 \text { mile above Sawmill Road, } \\
3 \text { miles east of Westminster, } \\
\text { Mass. }\end{array}$ & 1.28 & $\begin{array}{r}9-04-71 \\
9-09-71 \\
9-29-71 \\
10-19-71 \\
8-24-72 \\
9-25-72 \\
10-06-72 \\
9-11-73 \\
9-13-73\end{array}$ & $\begin{array}{c}.62 \\
.67 \\
.64 \\
.63 \\
1.0 \\
.88 \\
.81 \\
.71 \\
.60\end{array}$ \\
\hline 4 & $\begin{array}{l}01094355 \\
\text { Flag Brook }\end{array}$ & $\begin{array}{l}\text { Lat } 42033^{\prime} 31^{\prime \prime} \text {, long } 71050^{\prime} 42^{\prime \prime} \text {, } \\
\text { Worcester County, } 500 \text { feet below } \\
\text { rallroad trestle, } 2.5 \text { miles } \\
\text { southwest of Fitchburg, Mass. }\end{array}$ & -- & $\begin{array}{l}7-30-73 \\
8-27-73 \\
8-29-73\end{array}$ & $\begin{array}{l}7.8 \\
3.6 \\
6.0\end{array}$ \\
\hline 5 & $\begin{array}{l}01094380 \\
\text { Pin illips } \\
\text { Brook }\end{array}$ & $\begin{array}{l}\text { Lat } 42035^{\prime} 28 \text { ", long } 71051^{\prime} 41^{\prime \prime}, \\
\text { Worcester County, at Potato } \\
\text { Hill Rd., } 4.2 \text { miles northeast of } \\
\text { Westminster, Mass. }\end{array}$ & $-\infty$ & $3-19-73$ & 69 \\
\hline 6 & $\begin{array}{l}01094395 \\
\text { Phillips } \\
\text { Brook }\end{array}$ & $\begin{array}{l}\text { Lat } 42034^{\prime} 33^{\prime \prime}, \text { long } 71050^{\prime} 19^{\prime \prime} \text {, } \\
\text { Worcester County, } 800 \text { feet above } \\
\text { mouth, } 2 \text { miles southwest of } \\
\text { Fitchburg, Mass. }\end{array}$ & -- & $\begin{array}{l}7-30-73 \\
8-27-73 \\
8-29-73\end{array}$ & $\begin{array}{l}3.8 \\
3.4 \\
2.1\end{array}$ \\
\hline 7 & $\begin{array}{l}01094398 \\
\text { North Nashua } \\
\quad \text { River }\end{array}$ & $\begin{array}{l}\text { Lat } 42034^{\prime} 30^{\prime \prime}, \text { long } 71050^{\prime} 06^{\prime \prime} \text {, } \\
\text { Worcester County, at Depot St.., } \\
1.5 \text { miles southwest of Fitchburg, } \\
\text { Mass. }\end{array}$ & -- & $8-01-73$ & 31 \\
\hline 8 & $\begin{array}{l}01094430 \\
\text { Falulah } \\
\text { Brook }\end{array}$ & $\begin{array}{l}\text { Lat } 42035^{\prime} 22^{\prime \prime}, \text { long } 71046^{\prime} 54^{\prime \prime} \text {, } \\
\text { Worcester County, at Pear } 1 \text { St., } \\
0.4 \text { mile east of State Teachers } \\
\text { College, Fitchburg. Mass. }\end{array}$ & a12.0 & $\begin{array}{r}8-24-72 \\
9-25-72 \\
9-29-72 \\
10-05-72 \\
9-11-73 \\
9-13-73 \\
9-14-73 \\
10-02-73 \\
10-17-73\end{array}$ & $\begin{array}{c}.41 \\
.84 \\
.35 \\
.83 \\
.81 \\
.37 \\
.35 \\
1.2 \\
.53\end{array}$ \\
\hline
\end{tabular}


Table 6.--Stream sites and discharge measurements (Continued)

\begin{tabular}{|c|c|c|c|c|c|}
\hline $\begin{array}{l}\text { Map } \\
\text { no. }\end{array}$ & $\begin{array}{l}\text { Stream and } \\
\text { U.S. Geolog- } \\
\text { icai Survey } \\
\text { station no. }\end{array}$ & Location & $\begin{array}{l}\text { Orainage } \\
\text { area } \\
\left(m i^{2}\right)\end{array}$ & Date & $\begin{array}{c}\text { Discharge } \\
\left(\mathrm{ft}^{3} / \mathrm{s}\right)\end{array}$ \\
\hline 9 & $\begin{array}{l}01094440 \\
\text { Baker Brook }\end{array}$ & $\begin{array}{l}\text { Lat } 42033^{\prime} 41^{\prime \prime} \text {, long } 71045 \cdot 56 " \text {, } \\
\text { Worcester County, at Falulah Rd., } \\
2.5 \text { miles southeast of Fitchburg, } \\
\text { Mass. }\end{array}$ & 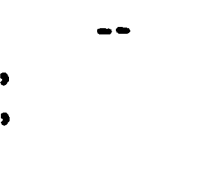 & $\begin{array}{l}7-30-73 \\
8-29-73\end{array}$ & $\begin{array}{l}2.8 \\
1.0\end{array}$ \\
\hline 10 & $\begin{array}{l}01094450 \\
\text { North Nashua } \\
\text { River }\end{array}$ & $\begin{array}{l}\text { Lat } 42^{\circ} 32^{\prime} 32^{\prime \prime} \text {, long } 71044 ' 47 " \text { ", } \\
\text { Worcester County, at Hamiltion } \\
\text { St., North Leominster. Mass. }\end{array}$ & -- & $\begin{array}{l}3-21-73 \\
1-08-74\end{array}$ & $\begin{array}{l}386 \\
170\end{array}$ \\
\hline 11 & $\begin{array}{l}01094460 \\
\text { Monoosnoc } \\
\text { Brook }\end{array}$ & $\begin{array}{l}\text { Lat } 42031^{\prime} 47^{\prime \prime} \text {, long } 71045 \text { '08", } \\
\text { Worcester County, at Whitney } \\
\text { St., Leominster, 2,500 feet } \\
\text { northeast of intersect ion of } \\
\text { Mechanic St. and Main St. }\end{array}$ & 11.0 & $\begin{array}{r}8-26-71 \\
9-27-71 \\
10-04-71 \\
10-20-71 \\
8-24-72 \\
9-28-72 \\
10-06-72 \\
3-19-73 \\
7-30-73 \\
8-29-73 \\
8-31-73 \\
9-12-73 \\
9-14-73 \\
10-02-73 \\
1-08-74\end{array}$ & $\begin{array}{c}1.3 \\
1.2 \\
1.1 \\
.98 \\
3.6 \\
1.7 \\
1.4 \\
94 \\
4.2 \\
2.6 \\
2.4 \\
2.3 \\
2.0 \\
1.7 \\
10\end{array}$ \\
\hline 12 & $\begin{array}{l}01094480 \\
\text { Fall Brook }\end{array}$ & $\begin{array}{l}\text { Lat } 42030 \text { '32", long } 71044 \text { ' } 43 " \text { ", } \\
\text { Worcester County, at Elm Hil1 } \\
\text { Ave., Leominster, 2,200 feet } \\
\text { above State Highway } 117 \text {. }\end{array}$ & 5.82 & $\begin{array}{r}8-26-71 \\
9-27-71 \\
10-01-71 \\
10-20-71 \\
8-24-72 \\
9-28-72 \\
10-06-72 \\
3-19-73 \\
8-31-73 \\
9-12-73 \\
9-14-73 \\
10-02-73 \\
1-08-74\end{array}$ & $\begin{array}{r}3.3 \\
6.3 \\
3.2 \\
2.7 \\
4.4 \\
7.5 \\
6.8 \\
51 \\
3.5 \\
2.7 \\
2.5 \\
2.9 \\
11\end{array}$ \\
\hline 13 & $\begin{array}{l}01094490 \\
\text { Fall Brook }\end{array}$ & $\begin{array}{l}\text { Lat } 42030^{\prime} 40^{\prime \prime} \text {, long } 71044 ' 20 " \text {, } \\
\text { Worcester County, at State } \\
\text { Highway } 117,1.5 \text { miles south- } \\
\text { east of Leominster, Mass. }\end{array}$ & -- & $\begin{array}{l}7-30-73 \\
8-29-73\end{array}$ & $\begin{array}{l}4.4 \\
3.8\end{array}$ \\
\hline 14 & $\begin{array}{l}01094500 \\
\text { North Nashua } \\
\text { River }\end{array}$ & $\begin{array}{l}\text { Lat } 42030^{\prime} 06^{\prime \prime}, \text { long } 71043^{\prime} 23^{\prime \prime}, \\
\text { Worcester County, } 1.3 \text { miles } \\
\text { upstream from Wekepeke Brook, } \\
2.5 \text { miles southeast of } \\
\text { Leominster. Gaging station } \\
\text { data available since September } \\
1935 \text { (see U.S. Geological Survey } \\
1954 \text {, 1964, 1966-75, and 1969). }\end{array}$ & al10 & & \\
\hline
\end{tabular}


Table 6.--Stream sites and discharge measurements (Cont inued)

\begin{tabular}{|c|c|c|c|c|c|}
\hline $\begin{array}{l}\text { Map } \\
\text { no. }\end{array}$ & $\begin{array}{l}\text { Stream and } \\
\text { U.S. Geolog- } \\
\text { ical Survey } \\
\text { station no. }\end{array}$ & Location & $\begin{array}{l}\text { Drainage } \\
\text { area } \\
\left(m i^{2}\right)\end{array}$ & Date & $\begin{array}{c}\text { Discharge } \\
\left(\mathrm{ft}^{3} / \mathrm{s}\right)\end{array}$ \\
\hline \multirow[t]{2}{*}{15} & $\begin{array}{l}01094550 \\
\text { Wekepeke } \\
\text { Brook }\end{array}$ & $\begin{array}{l}\text { Lat } 42029^{\prime} 19^{\prime \prime}, \text { long } 71042 ' 51 " \text {, } \\
\text { Worcester County, at State } \\
\text { Highway } 117,3 \text { miles northwest } \\
\text { of Lancaster, Mass. }\end{array}$ & 11.5 & $\begin{array}{r}8-26-71 \\
9-28-71 \\
10-01-71 \\
10-04-71 \\
10-22-71 \\
8-23-72 \\
9-25-72 \\
10-05-72 \\
7-31-73 \\
8-01-73 \\
8-29-73 \\
9-11-73 \\
9-13-73 \\
9-14-73 \\
9-14-73\end{array}$ & $\begin{array}{l}3.5 \\
3.5 \\
4.0 \\
3.3 \\
4.6 \\
4.7 \\
6.0 \\
4.7 \\
7.5 \\
8.7 \\
4.4 \\
4.1 \\
4.2 \\
4.5 \\
4.4\end{array}$ \\
\hline & & & & $\begin{array}{r}9-28-84 \\
10-11-84 \\
6-05-85 \\
7-25-85 \\
8-19-85 \\
9-18-85\end{array}$ & $\begin{array}{l}2.9 \\
4.2 \\
7.1 \\
2.9 \\
3.3 \\
3.6\end{array}$ \\
\hline 16 & $\begin{array}{l}01094700 \\
\text { North Nashua } \\
\text { River }\end{array}$ & $\begin{array}{l}\text { Lat } 42028 \text { '47", long } 71041 \text { '04", } \\
\text { Worcester County, } 600 \text { feet } \\
\text { downstream from bridge at } \\
\text { Ponak in Mil1, } 1.7 \text { miles north } \\
\text { of Lancaster, Mass. Water } \\
\text { qual ity monitor data since } \\
\text { October 1968: Specif ic con- } \\
\text { ductance, dissolved oxygen, } \\
\text { pH, and water temperature (see } \\
\text { U.S. Geological Survey } 1970-75) \text {. }\end{array}$ & al28 & & \\
\hline 17 & $\begin{array}{l}01094720 \\
\text { North Nashua } \\
\text { River }\end{array}$ & $\begin{array}{l}\text { Lat } 42028 ' 23^{\prime \prime} \text {, long } 71040 ' 59^{\prime \prime}, \\
\text { Worcester County, at North } \\
\text { Main St., } 1.5 \text { miles north } \\
\text { of Lancaster, Mass. }\end{array}$ & -- & $\begin{array}{l}3-21-73 \\
1-08-74\end{array}$ & $\begin{array}{l}549 \\
240\end{array}$ \\
\hline 18 & $\begin{array}{l}01094730 \\
\text { North Nashua } \\
\text { River }\end{array}$ & $\begin{array}{l}\text { Lat } 42027^{\prime} 04^{\prime \prime} \text {, long } 71040 ' 28 " \text {, } \\
\text { Worcester County, at Main St., } \\
0.5 \text { mile south of Lancaster, } \\
\text { Mass. }\end{array}$ & - & $\begin{array}{l}8-02-73 \\
8-28-73 \\
8-30-73\end{array}$ & $\begin{array}{r}106 \\
85 \\
76\end{array}$ \\
\hline
\end{tabular}


Table 6.--Stream sites and discharge measurements (Continued)

\begin{tabular}{|c|c|c|c|c|c|}
\hline $\begin{array}{l}\text { Map } \\
\text { no. }\end{array}$ & $\begin{array}{l}\text { Stream and } \\
\text { U.S. Geolog- } \\
\text { ical Survey } \\
\text { station no. }\end{array}$ & Loc ation & $\begin{array}{l}\text { Drainage } \\
\text { area } \\
\left(m i^{2}\right)\end{array}$ & Date & $\begin{array}{c}\text { Discharge } \\
\left(\mathrm{ft}^{3} / \mathrm{s}\right)\end{array}$ \\
\hline 19 & $\begin{array}{l}01094750 \\
\text { Waushacum } \\
\text { Brook }\end{array}$ & $\begin{array}{l}\text { Lat } 42024^{\prime} 34^{\prime \prime} \text {, long } 71045^{\prime} 56^{\prime \prime} \text {, } \\
\text { Worcester County, } 300 \text { feet be low } \\
\text { outlet of West Waushacum Pond, } \\
2 \text { miles south of Sterling, Mass. }\end{array}$ & 4.98 & $\begin{array}{r}8-26-71 \\
10-01-71 \\
10-20-71 \\
8-23-72 \\
9-27-72 \\
10-05-72 \\
9-11-73 \\
9-13-73 \\
9-14-73\end{array}$ & $\begin{array}{l}.03 \\
.09 \\
.26 \\
.55 \\
1.3 \\
.69 \\
.17 \\
.13 \\
.12\end{array}$ \\
\hline 20 & $\begin{array}{l}01094760 \\
\text { Waushacum } \\
\text { Brook }\end{array}$ & $\begin{array}{l}\text { Lat } 42^{\circ} 23^{\prime} 49^{\prime \prime}, \text { long } 71^{\circ} 46^{\prime} 48^{\prime \prime}, \\
\text { Worcester County, at Prescott } \\
\text { St., } 1.5 \text { miles north of intersec- } \\
\text { tion of State Highways 12, } 110 \text {, } \\
\text { and } 140, \text { in West Boylston, Mass. }\end{array}$ & 7.42 & $\begin{array}{r}8-26-71 \\
10-01-71 \\
10-20-71 \\
8-23-72 \\
9-27-72 \\
10-05-72 \\
3-19-73 \\
9-11-73 \\
9-13-73 \\
9-14-73 \\
1-08-74\end{array}$ & $\begin{array}{c}0.13 \\
.29 \\
.62 \\
.78 \\
1.7 \\
1.1 \\
46 \\
.48 \\
.37 \\
.37 \\
14\end{array}$ \\
\hline 21 & $\begin{array}{l}01095000 \\
\text { Rocky Brook }\end{array}$ & $\begin{array}{l}\text { Lat } 42026^{\prime} 57^{\prime \prime} \text {, long } 71048^{\prime} 10^{\prime \prime} \text {, } \\
\text { Worcester County, } 150 \text { feet down- } \\
\text { stream from bridge on Beaman Rd., } \\
2.2 \text { miles west of Sterling. Gag- } \\
\text { ing station data available for } \\
\text { October } 1946 \text { through September } \\
1967 \text { (see U.S. Geological Survey } \\
1954 \text {, 1964, 1966-68, and 1969). }\end{array}$ & 2.28 & $\begin{array}{l}9-27-84 \\
7-25-85 \\
8-19-85 \\
9-17-85\end{array}$ & $\begin{array}{l}.99 \\
.20 \\
.16 \\
.19\end{array}$ \\
\hline 22 & $\begin{array}{l}01095050 \\
\text { Stillwater } \\
\text { River }\end{array}$ & $\begin{array}{l}\text { Lat } 42026^{\prime} 00^{\prime \prime}, \text { long } 71^{\circ} 48^{\prime} 41^{\prime \prime} \text {, } \\
\text { Worcester County, at State High- } \\
\text { way } 62 \text {, Moores Corners, } 2.5 \text { miles } \\
\text { west of Sterling, Mass. }\end{array}$ & 25.7 & $\begin{array}{r}8-26-71 \\
9-09-71 \\
9-30-71 \\
10-20-71 \\
8-23-72 \\
9-28-72 \\
10-05-72 \\
9-11-73 \\
9-13-73 \\
9-27-84 \\
7-25-85 \\
8-19-85 \\
9-17-85\end{array}$ & $\begin{array}{l}1.9 \\
1.7 \\
3.2 \\
5.1 \\
4.4 \\
3.9 \\
3.7 \\
2.3 \\
1.9 \\
1.8 \\
1.5 \\
4.1 \\
4.8\end{array}$ \\
\hline
\end{tabular}


Table 6.--Stream sites and discharge measurements (Continued)

\begin{tabular}{|c|c|c|c|c|c|c|}
\hline $\begin{array}{l}\text { Map } \\
\text { no. }\end{array}$ & $\begin{array}{l}\text { Stream and } \\
\text { U.S. Geolog- } \\
\text { icai Survey } \\
\text { station no. }\end{array}$ & Locat io & & $\begin{array}{c}\text { Drainage } \\
\text { area } \\
\left(m i^{2}\right)\end{array}$ & Date & $\begin{array}{c}\text { Discharge } \\
\left(\mathrm{ft}^{3} / \mathrm{s}\right)\end{array}$ \\
\hline 23 & $\begin{array}{l}01095220 \\
\text { Stillwater } \\
\text { River }\end{array}$ & $\begin{array}{l}\text { Lat } 42024 \text { ' } 39 \text { ", long } 7 \\
\text { Worcester County, a } \\
\text { Rd., } 2.5 \text { miles sout } \\
\text { Sterling, Mass. }\end{array}$ & $\begin{array}{l}71047 \text { ' } 30 " \text { ", } \\
\text { huddy Pond } \\
\text { chwest of }\end{array}$ & 30.3 & $\begin{array}{r}8-26-71 \\
9-09-71 \\
9-30-71 \\
10-20-71 \\
8-23-72 \\
9-28-72 \\
10-05-72 \\
3-19-73 \\
9-11-73 \\
9-13-73 \\
1-08-74 \\
9-27-84 \\
7-25-85 \\
8-19-85 \\
9-17-85\end{array}$ & $\begin{array}{c}2.4 \\
3.2 \\
3.6 \\
5.6 \\
6.1 \\
5.7 \\
5.2 \\
275 \\
3.8 \\
3.5 \\
55 \\
3.1 \\
3.3 \\
5.9 \\
7\end{array}$ \\
\hline 24 & $\begin{array}{l}01095330 \\
\text { Qu inapoxet } \\
\text { River }\end{array}$ & $\begin{array}{l}\text { Lat } 42^{\circ} 22^{\prime} 50^{\prime \prime} \text {, long } 7 \\
\text { Worcester County, a } \\
2 \text { miles north of Ho } \\
\text { Mass. }\end{array}$ & $\begin{array}{l}1051^{\prime} 23 " \text { ", } \\
\text { at Mills St., } \\
\text { olden Center, }\end{array}$ & 21.8 & $\begin{array}{r}8-19-71 \\
9-28-71 \\
10-01-71 \\
10-19-71 \\
8-25-72 \\
9-27-72 \\
10-06-72 \\
9-12-73 \\
9-14-73\end{array}$ & $\begin{array}{l}.35 \\
.60 \\
.53 \\
.68 \\
.92 \\
.93 \\
.79 \\
1.2 \\
1.4\end{array}$ \\
\hline 25 & $\begin{array}{l}01095360 \\
\text { Asnebumsk it } \\
\text { Brook }\end{array}$ & $\begin{array}{l}\text { Lat } 42021 \text { ' } 40 \text { ", long } \\
\text { Worcester County, } \\
\text { Highway } 122 \mathrm{~A} \text {, Jeff } \\
\text { west of Holden Cen }\end{array}$ & $\begin{array}{l}71053^{\prime} 05^{\prime \prime}, \\
\text { at State } \\
\text { ferson, } 1 \text { mile } \\
\text { iter, Mass. }\end{array}$ & -- & $\begin{array}{r}8-19-71 \\
9-28-71 \\
10-01-71 \\
10-19-71 \\
8-25-72 \\
9-25-72 \\
9-29-72 \\
10-06-72 \\
9-12-73\end{array}$ & $\begin{array}{l}0.85 \\
.48 \\
.48 \\
.48 \\
.98 \\
.86 \\
.85 \\
.75 \\
.74\end{array}$ \\
\hline 26 & $\begin{array}{l}01095370 \\
\text { Asnebumsk it } \\
\text { Brook }\end{array}$ & $\begin{array}{l}\text { Lat } 42022 ' 24 \text { ", long } \\
\text { Worcester County, } \\
1.5 \text { miles north of } \\
\text { Mass. }\end{array}$ & $\begin{array}{l}71051 \text { '14", } \\
\text { at Mills St., } \\
\text { Holden Center, }\end{array}$ & -- & $\begin{array}{r}8-19-71 \\
9-28-71 \\
10-01-71 \\
10-19-71 \\
8-25-72 \\
9-25-72 \\
9-29-72 \\
10-06-72 \\
10-02-73\end{array}$ & $\begin{array}{l}2.0 \\
1.9 \\
1.7 \\
1.8 \\
3.9 \\
3.7 \\
3.1 \\
3.1 \\
2.9\end{array}$ \\
\hline
\end{tabular}


Table 6.--Stream sites and discharge measurements (Continued)

\begin{tabular}{|c|c|c|c|c|c|}
\hline $\begin{array}{l}\text { Map } \\
\text { no. }\end{array}$ & $\begin{array}{l}\text { Stream and } \\
\text { U.S. Geolog- } \\
\text { ical Survey } \\
\text { station no. }\end{array}$ & Location & $\begin{array}{c}\text { Drainage } \\
\text { area } \\
\quad\left(m i^{2}\right)\end{array}$ & Date & $\begin{array}{c}\text { Discharge } \\
\left(\mathrm{ft}^{3} / \mathrm{s}\right)\end{array}$ \\
\hline 27 & $\begin{array}{l}01095380 \\
\text { Trout Brook }\end{array}$ & $\begin{array}{l}\text { Lat } 42023^{\prime} 00^{\prime \prime}, \text { long } 71050 ' 12^{\prime \prime}, \\
\text { Worcester County, at Manning St., } \\
2.2 \text { miles north of Holden Center, } \\
\text { Mass. }\end{array}$ & 6.80 & $\begin{array}{r}8-26-71 \\
9-27-71 \\
9-29-71 \\
10-19-71 \\
8-23-72 \\
9-27-72 \\
10-05-72 \\
9-11-73 \\
9-13-73 \\
9-14-73\end{array}$ & $\begin{array}{c}0.14 \\
.31 \\
.44 \\
1.1 \\
.97 \\
1.2 \\
.96 \\
.45 \\
.43 \\
.43\end{array}$ \\
\hline 28 & $\begin{array}{l}01095400 \\
\text { Qu in apoxet } \\
\text { River }\end{array}$ & $\begin{array}{l}\text { Lat } 42023^{\prime} 11^{\prime \prime} \text {, long } 71048 \cdot 23^{\prime \prime} \text {, } \\
\text { Worcester County, } 0.5 \mathrm{mile} \text { up- } \\
\text { stream from mouth, } 0.6 \mathrm{mile} \text { west } \\
\text { of Oakdale, Mass. }\end{array}$ & -- & - & -- \\
\hline 29 & $\begin{array}{l}01095410 \\
\text { Malden Brook }\end{array}$ & $\begin{array}{l}\text { Lat } 42022^{\prime} 53^{\prime \prime}, \text { long } 71047^{\prime} 45^{\prime \prime}, \\
\text { Worcester County, at Thomas St., } \\
\text { Oakdale, } 0.5 \text { mile south of inter- } \\
\text { section of Thomas St. and State } \\
\text { Highway } 140 \text {. }\end{array}$ & 1.26 & $\begin{array}{r}8-19-71 \\
9-27-71 \\
9-30-71 \\
10-18-71 \\
8-23-72 \\
8-25-72 \\
9-25-72 \\
9-29-72 \\
10-05-72 \\
9-11-73 \\
9-13-73 \\
9-14-73\end{array}$ & $\begin{array}{l}.53 \\
.62 \\
.46 \\
.46 \\
.93 \\
.83 \\
.83 \\
.63 \\
.59 \\
.83 \\
.66 \\
.86\end{array}$ \\
\hline 30 & $\begin{array}{l}01095430 \\
\text { Gates Brook }\end{array}$ & $\begin{array}{l}\text { Lat } 42021^{\prime} 29^{\prime \prime} \text {, long } 71046^{\prime} 44^{\prime \prime} \text {, } \\
\text { Worcester County, at State High- } \\
\text { way } 140 \text {, West Boylston, } 0.2 \text { mile } \\
\text { south of intersection of State } \\
\text { Highways } 12 \text { and } 140 .\end{array}$ & 2.80 & $\begin{array}{r}8-19-71 \\
9-28-71 \\
10-01-71 \\
10-19-71 \\
8-25-72 \\
10-06-72 \\
9-12-73 \\
9-14-73\end{array}$ & $\begin{array}{l}0.41 \\
.57 \\
.51 \\
.80 \\
.52 \\
.63 \\
.60 \\
.57\end{array}$ \\
\hline
\end{tabular}

3101095500

South Branch Nashua River
Lat 42024'12", long 71041'19", Worcester County, at Wachusett Dam, 1 mile south of Clinton. Monthly bas in runoff data fur$n$ ished by Water Division of Metropolitan District Commission available since July 1896 (see U.S. Geological Survey 1954, 1964, 1966-75, 1969). 
Table 6.--Stream sites and discharge measurements (Cont inued)

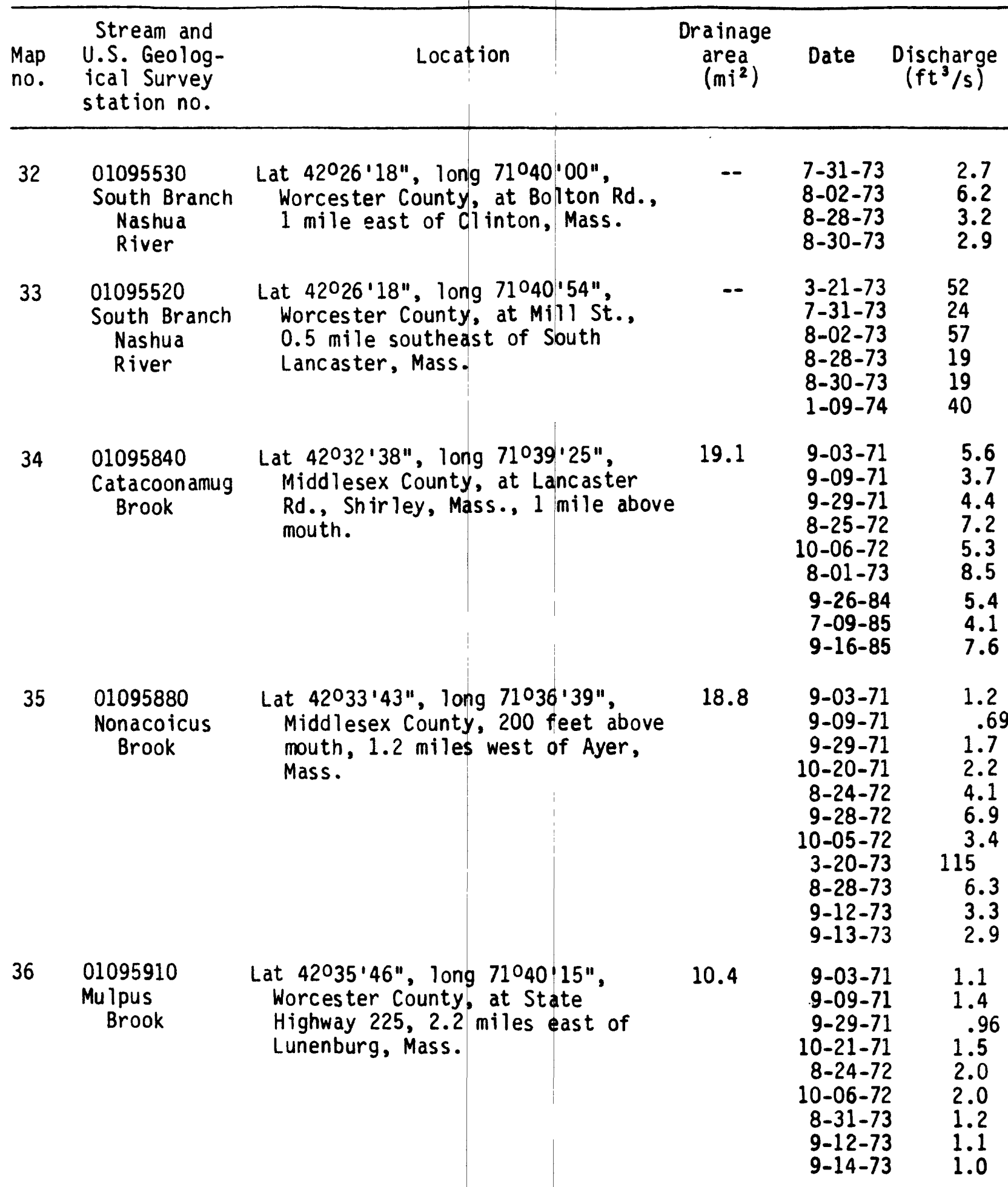


Table 6.--Stream sites and discharge measurements (Cont inued)

\begin{tabular}{|c|c|c|c|c|c|}
\hline $\begin{array}{l}\text { Map } \\
\text { no. }\end{array}$ & $\begin{array}{l}\text { Stream and } \\
\text { U.S. Geolog- } \\
\text { ical Survey } \\
\text { station no. }\end{array}$ & Location & $\begin{array}{c}\text { Drainage } \\
\text { area } \\
\left(m i^{2}\right)\end{array}$ & Date & $\begin{array}{c}\text { Discharge } \\
\left(\mathrm{ft}^{\circ} / \mathrm{s}\right)\end{array}$ \\
\hline 37 & $\begin{array}{l}01095915 \\
\text { Mulpus } \\
\text { Brook }\end{array}$ & $\begin{array}{l}\text { Lat } 42^{\circ} 34 \text { ' } 26 " \text { ", long } 71034 ' 43^{\prime \prime}, \\
\text { Middlesex County, at State } \\
\text { Highway } 2 \mathrm{~A}, 2.5 \text { miles } \\
\text { nor theast of Shirley, Mass. }\end{array}$ & 15.7 & $\begin{array}{r}9-03-71 \\
9-09-71 \\
9-29-71 \\
10-21-71 \\
8-24-72 \\
8-24-72 \\
9-25-72 \\
10-06-72 \\
3-20-73 \\
8-01-73 \\
8-31-73 \\
9-12-73 \\
1-09-74\end{array}$ & $\begin{array}{c}1.1 \\
1.1 \\
1.5 \\
1.9 \\
3.9 \\
4.1 \\
3.9 \\
3.0 \\
128 \\
5.4 \\
2.3 \\
2.0 \\
30\end{array}$ \\
\hline 38 & $\begin{array}{l}01095920 \\
\text { Nashua River }\end{array}$ & $\begin{array}{l}\text { Lat } 42034^{\prime} 42 " \text {, long } 71036135 " \text { ", } \\
\text { Middlesex County, at State High- } \\
\text { way } 2 A, 1.5 \text { miles northwest of } \\
\text { Ayer, Mass. }\end{array}$ & - & $\begin{array}{l}3-21-73 \\
1-09-74\end{array}$ & $\begin{array}{r}1,130 \\
290\end{array}$ \\
\hline 39 & $\begin{array}{c}01095930 \\
\text { Will ard } \\
\text { Brook }\end{array}$ & $\begin{array}{l}\text { Lat } 42040^{\prime} 27^{\prime \prime} \text {, long } 71046^{\prime} 14^{\prime \prime} \text {, } \\
\text { Middlesex County, at State High- } \\
\text { way } 119,1,500 \mathrm{feet} \text { west of in- } \\
\text { tersection of State Highway } 119 \\
\text { and Whee ler Rd., 1.5 miles west } \\
\text { of West Townsend, Mass. }\end{array}$ & a12.3 & $\begin{array}{r}9-04-71 \\
9-27-71 \\
10-01-71 \\
10-22-71 \\
9-26-72 \\
10-05-72 \\
9-11-73 \\
9-13-73\end{array}$ & $\begin{array}{l}1.4 \\
1.2 \\
.94 \\
1.6 \\
1.9 \\
1.5 \\
1.8 \\
1.6\end{array}$ \\
\hline \multirow[t]{2}{*}{40} & $\begin{array}{l}01095940 \\
\text { Locke Brook }\end{array}$ & $\begin{array}{l}\text { Lat } 42^{\circ} 40^{\prime} 42^{\prime \prime} \text {, long } 71045^{\prime} 31^{\prime \prime} \text {, } \\
\text { Middlesex County, at West Meadow } \\
\text { Rd., West Townsend, Mass., } \\
0.5^{2} \text { mile above mouth. }\end{array}$ & 4.20 & $\begin{array}{r}9-04-71 \\
10-22-71 \\
9-02-72 \\
10-05-72\end{array}$ & $\begin{array}{l}0 \\
.43 \\
b .2 \\
b .04\end{array}$ \\
\hline & & & & $9-17-85$ & 0 \\
\hline \multirow[t]{2}{*}{41} & $\begin{array}{l}01095950 \\
\text { Mason Brook }\end{array}$ & $\begin{array}{l}\text { Lat } 42042 \cdot 05^{\prime \prime} \text {. long } 710^{\circ} 45^{\prime} 10^{\prime \prime}, \\
\text { Middlesex County, } 2,200 \text { feet } \\
\text { south of New Hampshire State } \\
\text { line and } 2 \text { miles northwest of } \\
\text { West Townsend, Mass. }\end{array}$ & 9.30 & $\begin{array}{r}9-03-71 \\
9-27-71 \\
10-04-71 \\
10-22-71 \\
8-23-72 \\
9-26-72 \\
10-05-72 \\
9-11-73 \\
9-13-73\end{array}$ & $\begin{array}{l}.05 \\
.19 \\
.11 \\
.53 \\
.42 \\
.21 \\
.16 \\
.31 \\
.21\end{array}$ \\
\hline & & & & $\begin{array}{l}6-10-85 \\
7-25-85 \\
8-23-85 \\
9-17-85\end{array}$ & $\begin{array}{l}1.7 \\
.18 \\
.11 \\
.43\end{array}$ \\
\hline
\end{tabular}


Table 6.--Stream sites ana oiscnarge measurements (Cont Inued)

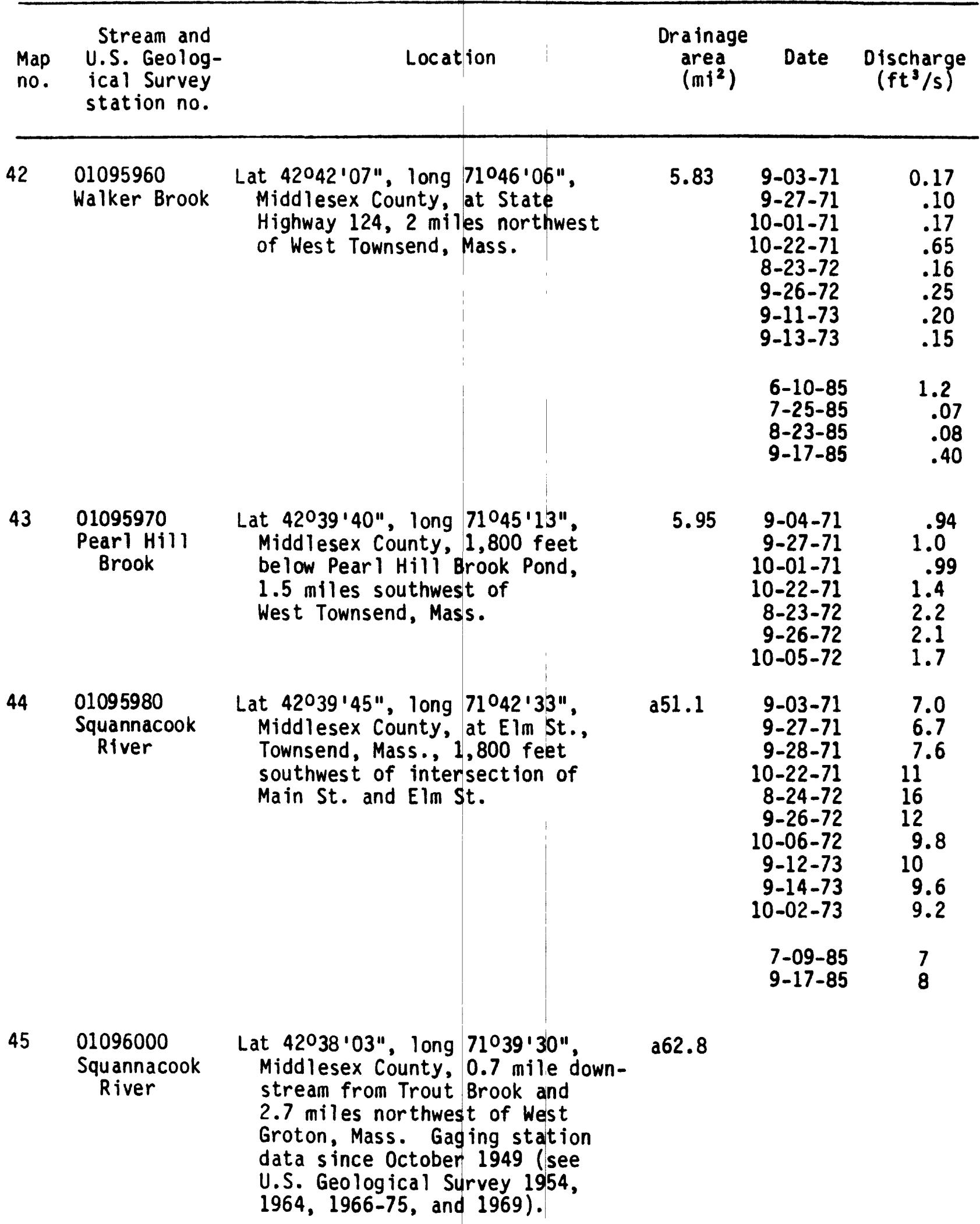


Table 6.--Stream sites and discharge measurements (Continued)

\begin{tabular}{|c|c|c|c|c|c|}
\hline $\begin{array}{l}\text { Map } \\
\text { no. }\end{array}$ & $\begin{array}{l}\text { Stream and } \\
\text { U.S. Geolog- } \\
\text { ical Survey } \\
\text { station no. }\end{array}$ & Location & $\begin{array}{l}\text { Drainag } \\
\text { area } \\
\left(m i^{2}\right)\end{array}$ & Date & $\begin{array}{c}\text { Discharge } \\
\left(\mathrm{ft}^{\circ} / \mathrm{s}\right)\end{array}$ \\
\hline 46 & $\begin{array}{l}01096040 \\
\text { Squannacook } \\
\text { River }\end{array}$ & $\begin{array}{l}\text { Lat } 42^{\circ} 35^{\prime} 20^{\prime \prime} \text {, long } 71^{0} 037^{\prime} 20^{\prime \prime} \text {, } \\
\text { Middlesex County, } 800 \text { feet below } \\
\text { Kittredge Rd., } 3.5 \text { miles north- } \\
\text { east of Shirley, Mass. }\end{array}$ & - & $\begin{array}{l}7-31-73 \\
8-28-73 \\
8-30-73\end{array}$ & $\begin{array}{l}29 \\
21 \\
19\end{array}$ \\
\hline 47 & $\begin{array}{l}01096050 \\
\text { Squannacook } \\
\text { River }\end{array}$ & $\begin{array}{l}\text { Lat } 42034^{\prime} 56^{\prime \prime} \text {, long } 71036^{\prime} 37^{\prime \prime} \text {, } \\
\text { Middlesex County, } 800 \text { feet } \\
\text { upstream from mouth, } 1.8 \text { miles } \\
\text { northwest of Ayer, Mass. }\end{array}$ & -- & -- & -- \\
\hline 8 & $\begin{array}{l}01096200 \\
\text { James Brook }\end{array}$ & $\begin{array}{l}\text { Lat } 42^{\circ} 34^{\prime} 46^{\prime \prime} \text {, long } 71035^{\prime} 20^{\prime \prime}, \\
\text { Middlesex County, at State } \\
\text { Highway 111, } 1.5 \text { miles } \\
\text { north of Ayer, Mass. }\end{array}$ & 3.91 & $\begin{array}{r}9-03-71 \\
9-09-71 \\
9-29-71 \\
10-21-71 \\
8-24-72 \\
9-25-72 \\
10-06-72 \\
8-31-73 \\
9-12-73 \\
9-13-73 \\
9-14-73\end{array}$ & $\begin{array}{l}0.05 \\
.08 \\
.24 \\
.73 \\
.62 \\
.70 \\
.61 \\
.15 \\
.13 \\
.10 \\
.11\end{array}$ \\
\hline 19 & $\begin{array}{l}01096300 \\
\text { Rob inson } \\
\text { Brook }\end{array}$ & $\begin{array}{l}\text { Lat } 42037 \text { '52", long } 71036 \text { ' } 32^{\prime \prime} \text {, } \\
\text { Middlesex County, at Shirley St., } \\
2.5 \text { miles southwest of Pepperell, } \\
\text { Mass. }\end{array}$ & 3.09 & $\begin{array}{r}9-03-71 \\
9-09-71 \\
9-28-71 \\
10-22-71 \\
8-23-72 \\
9-26-72 \\
8-31-73 \\
9-11-73 \\
9-13-73\end{array}$ & $\begin{array}{c}.10 \\
.12 \\
.08 \\
.18 \\
.42 \\
1.2 \\
.12 \\
.21 \\
.22\end{array}$ \\
\hline
\end{tabular}

$50 \quad 01096500$ Nashua

River

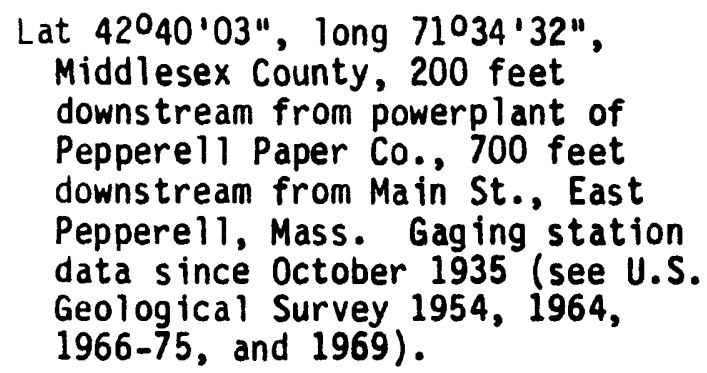


Table 6.--Stream sites and discharge measurements (Continued)

\begin{tabular}{|c|c|c|c|c|c|c|}
\hline $\begin{array}{l}\text { Map } \\
\text { no. }\end{array}$ & $\begin{array}{l}\text { Stream and } \\
\text { U.S. Geolog- } \\
\text { ical Survey } \\
\text { station no. }\end{array}$ & Locat & ion & $\begin{array}{c}\text { Drainage } \\
\text { area } \\
\left(m i^{2}\right)\end{array}$ & Date & $\begin{array}{c}\text { Discharge } \\
(\mathrm{ft} / \mathrm{s})\end{array}$ \\
\hline 51 & $\begin{array}{l}01096502 \\
\text { Nissitiss it } \\
\quad \text { River }\end{array}$ & $\begin{array}{l}\text { Lat } 422^{\circ} 42^{\prime} 20^{\prime \prime}, \text { long } \\
\text { Hillsborough Count } \\
500 \text { feet upstream } \\
\text { Pepperell Rd. } 3 \mathrm{~m} \\
\text { southwest of Holli }\end{array}$ & $\begin{array}{l}71037 \cdot 18 \text { ", } \\
\text { ty, N.H., } \\
\text { from } \\
\text { miles } \\
\text { is, N.H. }\end{array}$ & 47.6 & $\begin{array}{r}9-03-71 \\
9-27-71 \\
10-04-71 \\
10-21-71 \\
8-23-72 \\
9-28-72 \\
10-05-72 \\
9-11-73 \\
9-13-73\end{array}$ & $\begin{array}{l}3.7 \\
3.2 \\
2.7 \\
7.1 \\
5.1 \\
6.7 \\
7.0 \\
6.6 \\
5.8\end{array}$ \\
\hline 52 & $\begin{array}{l}01096503 \\
\text { Niss itiss it } \\
\quad \text { River }\end{array}$ & $\begin{array}{l}\text { Lat } 42040 ' 19 " \text {, long } \\
\text { Middlesex County, } \\
\text { Peppere } 11 \text {, Mass., } \\
\text { above mouth. }\end{array}$ & $\begin{array}{l}71034: 39 ", \\
\text { at Canal St., } \\
4,000 \text { feet }\end{array}$ & 59.6 & $\begin{array}{r}8-27-71 \\
9-27-71 \\
10-04-71 \\
10-21-71 \\
8-23-72 \\
9-28-72 \\
10-05-72 \\
3-22-73 \\
7-31-73 \\
8-28-73 \\
8-30-73 \\
9-13-73 \\
1-10-74\end{array}$ & $\begin{array}{c}3.5 \\
4.9 \\
3.9 \\
7.6 \\
8.8 \\
11 \\
8.6 \\
303 \\
30 \\
10 \\
9.5 \\
8.5 \\
90\end{array}$ \\
\hline 53 & $\begin{array}{l}01096504 \\
\text { Reedy Meadow } \\
\text { Brook }\end{array}$ & $\begin{array}{l}\text { Lat } 42040^{\prime} 03^{\prime \prime} \text {, long } \\
\text { Middlesex County, } \\
\text { East Peppere11, Ma } \\
\text { above mouth. }\end{array}$ & $\begin{array}{l}71033^{\prime} 55^{\prime \prime}, \\
\text { at Lowe } 11 \text { 'Rd., } \\
\text { ass., 2,000 feet }\end{array}$ & 1.94 & $\begin{array}{r}8-27-71 \\
9-09-71 \\
9-29-71 \\
10-04-71 \\
10-21-71 \\
8-23-72 \\
9-26-72 \\
10-05-72 \\
8-31-73 \\
9-13-73\end{array}$ & $\begin{array}{l}.66 \\
.46 \\
.74 \\
.58 \\
.64 \\
1.5 \\
.96 \\
.89 \\
.80 \\
.64\end{array}$ \\
\hline
\end{tabular}


Table 6.--Stream sites and discharge measurements (Continued)

\begin{tabular}{|c|c|c|c|c|c|}
\hline $\begin{array}{l}\text { Map } \\
\text { no. }\end{array}$ & $\begin{array}{l}\text { Stream and } \\
\text { U.S. Geolog- } \\
\text { ical Survey } \\
\text { station no. }\end{array}$ & Location & $\begin{array}{l}\text { Drainage } \\
\text { area } \\
\left(m i^{2}\right)\end{array}$ & Date & $\begin{array}{c}\text { Discharge } \\
\left(\mathrm{ft}^{3} / \mathrm{s}\right)\end{array}$ \\
\hline 54 & $\begin{array}{l}01096505 \\
\text { Unkety Brook }\end{array}$ & $\begin{array}{l}\text { Lat } 42^{\circ} 41^{\prime} 23^{\prime \prime}, \text { long } 71032 \cdot 54^{\prime \prime}, \\
\text { Middlesex County, at River St., } \\
2.5 \text { miles northeast of Peppereit, } \\
\text { Mass. }\end{array}$ & 6.84 & $\begin{array}{r}8-27-71 \\
9-09-71 \\
9-29-71 \\
10-04-71 \\
10-21-71 \\
8-23-72 \\
9-26-72 \\
10-05-72 \\
3-21-73 \\
7-31-73 \\
8-01-73 \\
8-28-73 \\
8-30-73 \\
1-10-74\end{array}$ & $\begin{array}{l}.93 \\
1.1 \\
1.8 \\
1.1 \\
1.8 \\
2.7 \\
2.4 \\
2.1 \\
27 \\
3.6 \\
3.2 \\
2.3 \\
1.4 \\
6\end{array}$ \\
\hline
\end{tabular}

a Net area (adjusted for diversions).

b Field estimate. 


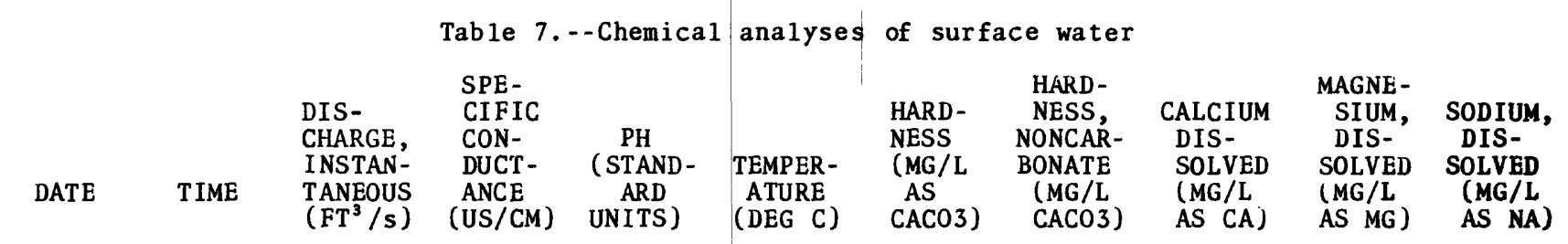

01094340 WHITMAN RIVER NEAR WESTMINSTER, MASS. (LAT 4233 35N LONG 0715202 W)

\begin{tabular}{|c|c|c|c|c|c|c|c|c|c|c|}
\hline $\begin{array}{l}\text { MAR } 1973 \\
19 . \\
\text { JAN } 1974\end{array}$ & 1640 & 173 & 56 & 5.9 & 5.0 & 10 & 7 & 3.2 & 0.6 & 5.3 \\
\hline $08 \ldots$ & 1245 & 40 & 69 & 5.7 & .0 & 11 & 10 & 3.4 & .7 & 6.7 \\
\hline
\end{tabular}

01094380 PHILLIPS BROOK NEAR WESTMINSTER, MASS. (LAT 4235 28N LONG $0715141 W$ )

\begin{tabular}{|c|c|c|c|c|c|c|c|c|c|c|}
\hline $\begin{array}{l}\text { MAR } \\
19 . \cdots \\
\text { JAN } 1974\end{array}$ & 1550 & 69 & 54 & 6.3 & 4.0 & 13 & 11 & 4.5 & 0.5 & .5 .8 \\
\hline $08 \ldots$ & 1330 & e20 & 67 & 6.2 & .0 & 10 & 8 & 3.0 & .7 & 6.7 \\
\hline
\end{tabular}

01094450 NORTH NASHUA RIVER AT NORTH LEOMINSTER, MASS. (LAT $423232 \mathrm{~N}$ LONG 0714447 W)

\begin{tabular}{|c|c|c|c|c|c|c|c|c|c|c|}
\hline $\begin{array}{l}\text { MAR } 1973 \\
21.2 \\
\text { JAN } 1974\end{array}$ & 0945 & 386 & 121 & 6.2 & 4.0 & 20 & 13 & 6.5 & 1.0 & 13 \\
\hline $08 \ldots$ & 1400 & 170 & 158 & 6.0 & 1.0 & 23 & 16 & 7.0 & 1.3 & 17 \\
\hline
\end{tabular}

01094460 MONOOSNOC BROOK AT LEOMINSTER, MASS. (LAT 423147 N LONG $0714508 W$ )

\begin{tabular}{|c|c|c|c|c|c|c|c|c|c|c|}
\hline $\begin{array}{l}\text { MAR } 1973 \\
19 . \\
\text { JAN } 1974\end{array}$ & 1430 & 94 & 52 & 6.1 & 5.0 & 10 & 8 & 3.5 & 0.4 & 5.2 \\
\hline $08 \ldots$ & 1440 & 10 & 133 & 6.5 & 1.0 & 17 & 12 & 5.4 & .8 & 17 \\
\hline
\end{tabular}

01094480 FALL BROOK AT LEOMINSTER, MASS. (LAT $42 \quad 30 \quad 32 \mathrm{~N}$ LONG $0714443 W$ )

\begin{tabular}{|c|c|c|c|c|c|c|c|c|c|c|}
\hline $\begin{array}{l}\text { MAR } 1973 \\
19 . \\
\text { JAN }\end{array}$ & 1330 & 51 & 89 & 6.7 & 7.0 & 22 & 12 & 7.0 & 1.0 & 6.9 \\
\hline $08 \ldots$ & 1510 & 11 & 181 & 6.9 & 1.0 & 47 & 31 & 16 & 1.8 & 14 \\
\hline
\end{tabular}

01094720 NORTH NASHUA RIVER AT N MAIN ST NR LANCASTER, MASS. (LAT $422823 \mathrm{~N}$ LONG 07140 59W) MAR 1973

\begin{tabular}{|c|c|c|c|c|c|c|c|c|c|}
\hline 21.974 & 1100 & 549 & 114 & 6.5 & 4.0 & 19 & 12 & 6.0 & 1.0 \\
\hline $08 \ldots$ & 1550 & 240 & 153 & 6.3 & .0 & 24 & 10 & 7.3 & 1.3 \\
\hline
\end{tabular}

01094760 WAUSHACUM BROOK NEAR WEST BOYLSTON, MASS. (LAT $422349 \mathrm{~N}$ LONG 0714648 W)

\begin{tabular}{|c|c|c|c|c|c|c|c|c|c|c|}
\hline $\begin{array}{l}\text { MAR } 1973 \\
19 . \\
\text { JAN } 1974\end{array}$ & 1230 & 46 & 136 & 6.7 & 6.0 & 30 & 18 & 9.8 & 1.4 & 14 \\
\hline $08 \ldots$ & 1115 & 14 & 165 & 6.7 & .0 & 33 & 18 & 10 & 1.9 & 16 \\
\hline
\end{tabular}

01095220 STILLWATER RIVER NEAR STERLING, MASS. (LAT $422439 \mathrm{~N}$ LONG $0714730 \mathrm{~W})$

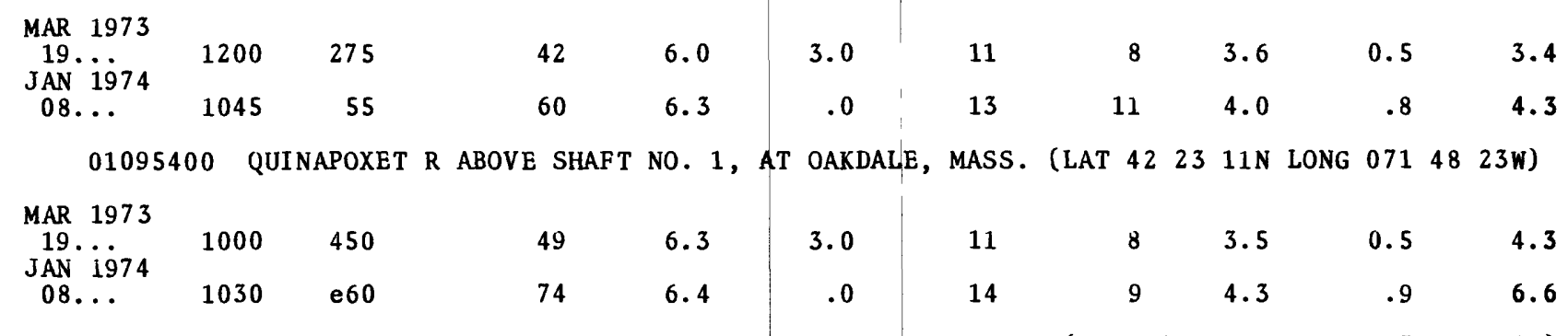

01095520 SOUTH BRANCH NASHUA RIVER AT SOUTH LANCASTER, MASS. (LAT 4226 18N LONG $0714054 W$ )

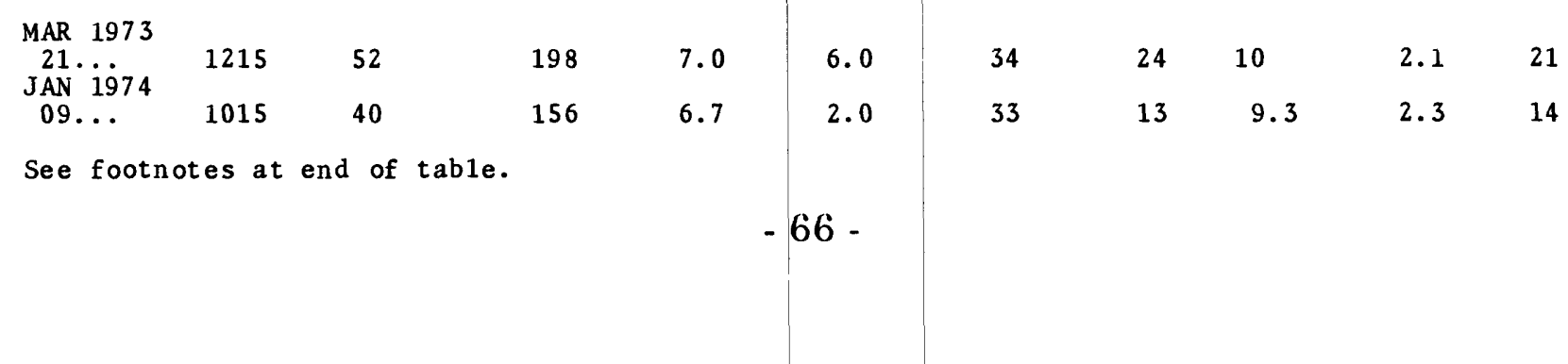


Table 7.--Chemical analyses of surface water (Continued)

\begin{tabular}{|c|c|c|c|c|c|c|c|c|c|c|}
\hline ATE & $\begin{array}{l}\text { POTAS- } \\
\text { SIUM, } \\
\text { DIS- } \\
\text { SOLVED } \\
\text { (MG/L }\end{array}$ & $\begin{array}{c}\text { BICAR- } \\
\text { BONATE } \\
\text { FET-FLD } \\
\text { (MG/L } \\
\text { AS }\end{array}$ & $\begin{array}{c}\text { ALKA- } \\
\text { LINITY } \\
\text { FIE LD } \\
\text { (MG/L } \\
\text { AS }\end{array}$ & $\begin{array}{l}\text { SULFATE } \\
\text { DIS- } \\
\text { SOLVED } \\
\text { (MG/L }\end{array}$ & $\begin{array}{l}\text { CHLO- } \\
\text { RIDE, } \\
\text { DIS- } \\
\text { SOLVED } \\
\text { (MG/L }\end{array}$ & $\begin{array}{l}\text { FLUO- } \\
\text { RIDE, } \\
\text { DIS- } \\
\text { SOLVED } \\
\text { (MG/L }\end{array}$ & $\begin{array}{l}\text { SI LICA, } \\
\text { DIS- } \\
\text { SOLVED } \\
\text { (MG/L } \\
\text { AS }\end{array}$ & $\begin{array}{c}\text { SOL IDS, } \\
\text { SUM OF } \\
\text { CONST I- } \\
\text { TUENTS, } \\
\text { DIS- } \\
\text { SOLVED }\end{array}$ & $\begin{array}{c}\text { NI TRO- } \\
\text { GEN, } \\
\text { N I TRATE } \\
\text { TOTAL } \\
\text { (MG/L }\end{array}$ & $\begin{array}{c}\text { NITRO- } \\
\text { GEN, } \\
\text { NITRATE } \\
\text { DIS- } \\
\text { SOLVED } \\
\text { (MG / }\end{array}$ \\
\hline & & & & AS SO4) & & & & & AS $N$ ) & AS N) \\
\hline
\end{tabular}

01094340 WHITMAN RIVER NEAR WESTMINSTER, MASS. (LAT $4233 \quad 35$ N LONG $0715202 W$ )

MAR 1973

19 ...

JAN 1974

$\begin{array}{rrrrrrrrrr}0.7 & 4 & 3 & 9.5 & 5.5 & 0.1 & 5.0 & 33 & -- & 0.20 \\ .9 & 1 & 1 & 8.7 & 11 & .2 & 5.2 & 37 & 0.23 & --\end{array}$

01094380 PHILLIPS BROOK NEAR WESTMINSTER, MASS. (LAT 4235 28N LONG $0715141 W$ )

MAR 1973

JAN 1974

$\begin{array}{llllll}0.6 & 2 & 2 & 7.9 & 8.5 & 0.1\end{array}$

0.1

4.9

35

0.20

08 ...

.8

$\begin{array}{llll}3 & 2 & 7.5 & 10\end{array}$

$5.9 \quad 36$

0.37

01094450 NORTH NASHUA RIVER AT NORTH LEOMINSTER, MASS. (LAT 423232 N LONG $0714447 W$ )

MAR 1973

$21 \ldots$

JAN 1974

08...

$\begin{array}{lllll}1.7 & 9 & 7 & 15 & 19\end{array}$

$19 \quad 0.2$

$5.6 \quad 69$

0.50

2.1

$\begin{array}{llll}8 & 7 & 21 & 23\end{array}$

.2

6.0

82

0.61

01094460 MONOOSNOC BROOK AT LEOMINSTER, MASS. (LAT 4231 47N LONG $0714508 W$ )

MAR 1973

$19 . .$.

JAN 1974

08 ...

$\begin{array}{lllll}0.5 & 3 & 2 & 7.7 & 10 \\ 1.1 & 6 & 5 & 9.4 & 27\end{array}$

0.1

5.2

35

$-0.10$

01094480 FALL BROOK AT LEOMINSTER, MASS. (LAT $42 \quad 30 \quad 32 N$ LONG $07144 \quad 43 W$ )

$\begin{array}{rrrrrrrrrr}\text { MAR } 1973 \\ \begin{array}{l}19 \\ \text { JAN } 1974\end{array} & 1.6 & 12 & 10 & 11 & 11 & 0.1 & 5.7 & 53 & \ldots .50 \\ 08 . & 2.2 & 19 & 16 & 14 & 31 & .1 & 6.4 & 95 & 0.59\end{array}$

01094720 NORTH NASHUA RIVER AT N MAIN ST NR LANCASTER, MASS. (LAT $422823 \mathrm{~N}$ LONG $0714059 \mathrm{~W})$

MAR 1973

21

JAN 1974

$\begin{array}{rrrrrrrrrr}1.8 & 9 & 7 & 14 & 20 & 0.1 & 6.0 & 69 & -. & 0.40 \\ 2.0 & 17 & 14 & 18 & 21 & .2 & 6.4 & 81 & 0.64 & \ldots\end{array}$

01094760 WAUSHACUM BROOK NEAR WEST BOYLSTON, MASS. (LAT $422349 \mathrm{~N}$ LONG 0714648 )

MAR 1973

JAN 1974

$1.5 \quad 15$

$12 \quad 12 \quad 24$

$<0.1$

5.2

76

$-\cdot$

0.20

08...

$\begin{array}{lllll}1.7 & 18 & 15 & 12 & 28\end{array}$

.1

4.5

83

0.24

01095220 STILLWATER RIVER NEAR STERLING, MASS. (LAT $42 \quad 24$ 39N LONG 0714730 W)

MAR 1973

$19 . .$.

JAN 1974

$0.7 \quad 4 \quad 3$

8.0

5.5

0.1

$4.6 \quad 29$

0.20

08...

$\begin{array}{lll}.9 & 3 & 2\end{array}$

$8.6 \quad 6.9$

.2

6.3

33

0.44

01095400 QUINAPOXET R ABOVE SHAFT NO. 1, AT OAKDALE, MASS. (LAT $422311 \mathrm{~N}$ LONG O71 $4823 W$ )

MAR 1973

$19 . .$.

JAN 1974

0.8

$4 \quad 3$

8.1

$8.2<0.1$

4.6

33

-

0.20

08...

1.0

$5 \quad 10$

11

$<.1$

5.7

42

0.29

01095520 SOUTH BRANCH NASHUA RIVER AT SOUTH LANCASTER, MASS. (LAT $42 \quad 26$ I8N LONG 07140 54W) MAR 1973

JAN 1974

$09 .$.

$\begin{array}{lllll}2.2 & 12 & 10 & 14 & 33 \\ 2.4 & 24 & 20 & 14 & 22\end{array}$

2.4

24

22

0.2
.4

7.0

5.9

110

$82 \quad 1.86$ 
Table 7.--Chemica1 analyses of surface water (Continued)

\begin{tabular}{|c|c|c|c|c|c|c|c|c|c|c|}
\hline ATE & $\begin{array}{l}\text { NITRO- } \\
\text { GEN, } \\
\text { NITR ITE } \\
\text { TOTAL } \\
\text { (MG/L } \\
\text { AS N) }\end{array}$ & $\begin{array}{c}\text { NI TRO- } \\
\text { GEN, } \\
\text { NI TRITE } \\
\text { DIS- } \\
\text { SOLVED } \\
\text { (MG/L } \\
\text { AS N) }\end{array}$ & $\begin{array}{c}\text { NITRO- } \\
\text { GEN, } \\
\text { NO2+NO3 } \\
\text { TOTAL } \\
\text { (MG/L } \\
\text { AS N) }\end{array}$ & $\begin{array}{c}\text { NI TRO- } \\
\text { GEN, } \\
\text { NO2 + NO3 } \\
\text { DIS- } \\
\text { SOLVED } \\
\text { (MG/L } \\
\text { AS N) }\end{array}$ & $\begin{array}{c}\text { NITRO- } \\
\text { GEN, } \\
\text { AMMONIA } \\
\text { TOTAL } \\
\text { (MG /L } \\
\text { AS N) }\end{array}$ & $\begin{array}{l}\text { NI TRO- } \\
\text { GEN, } \\
\text { AMMONIA } \\
\text { DIS- } \\
\text { SOLVED } \\
\text { (MG/L } \\
\text { AS N) }\end{array}$ & $\begin{array}{c}\text { NITRO- } \\
\text { GEN, } \\
\text { ORGAN IC } \\
\text { TOTAL } \\
\text { (MG/L } \\
\text { AS N) }\end{array}$ & $\begin{array}{c}\text { PHOS- } \\
\text { PHORUS, } \\
\text { TOTAL } \\
\text { (MG/L } \\
\text { AS P) }\end{array}$ & $\begin{array}{l}\text { IRON, } \\
\text { TOTAL } \\
\text { RECOV- } \\
\text { ERABLE } \\
\text { (UG/L } \\
\text { AS FE) }\end{array}$ & $\begin{array}{l}\text { MANGA- } \\
\text { NESE, } \\
\text { TOTAL } \\
\text { RECOV- } \\
\text { ERABLE } \\
\text { (UG/L } \\
\text { AS MN) }\end{array}$ \\
\hline
\end{tabular}

01094340 WHITMAN RIVER NEAR WESTMINSTER, MASS. (LAT $42 \quad 33 \quad 35$ N LONG $07152 \quad 02$ W)

MAR 1973

JAN 1974

08 ...

\begin{tabular}{|c|c|c|c|c|c|c|c|c|}
\hline-- & 0.005 & - & $\cdots$ & - & 0.07 & 0.66 & 0.018 & 260 \\
\hline$<0.01$ & $\cdots$ & 0.23 & $\ldots$ & - & -. & -. & -. & 390 \\
\hline
\end{tabular}

01094380 PHILLIPS BROOK NEAR WESTMINSTER, MASS. (LAT 4235 28N LONG $0715141 W$ )

MAR 1973

JAN 1974

08 ...
-- $\quad 0.007$
0.
$-\quad 0.07$
0.44
0.011
160
70
0.01
$\begin{array}{ll}- & 0.38\end{array}$
$--$
- -

-- 230
60

01094450 NORTH NASHUA RIVER AT NORTH LEOMINSTER, MASS. (LAT 423232 N LONG 0714447 ) MAR 1973 JAN 1974
$08 \ldots$
0.01
0.025
\begin{tabular}{c|c|c}
- & - & 0.39 \\
- & 0.88 &
\end{tabular}
0.76
0.27
530
110

01094460 MONOOSNOC BROOK AT LEOMINSTER, MASS. (LAT $423147 \mathrm{~N}$ LONG 07145 O8W)

MAR 1973

19

JAN 1974

$08 \ldots$
- $\quad 0.004$
-
0.06
0.11
0.046
250
120
0.01
0.29
--
-
- 250
110

01094480 FALL BROOK AT LEOMINSTER, MASS, (LAT $423032 \mathrm{~N}$ LONG $0714443 W$ )

MAR 1973

JAN 1974
0.01
0.60
0.07
0.26
0.024
400
90
0.01
$-$
\begin{tabular}{l|l} 
& 0.07 \\
- & -
\end{tabular}
-. $\quad 320$
90

08 ...

01094720 NORTH NASHUA RIVER AT N MAIN ST NR LANCA\$TER, MASS. (LAT $422823 \mathrm{~N}$ LONG $0714059 W$ ) MAR 1973

21.
JAN 1974
$08 \ldots$
0.045

$--$

0.20

0.35

0.19

590

110

0.01

-. 0.65

1.30

-

-

-

870

130

01094760 WAUSHACUM BROOK NEAR WEST BOYLSTON, MASS. (LAT $422349 \mathrm{~N}$ LONG $0714648 \mathrm{~W}$ )

MAR 1973

MAR 1973
$19 . \cdots$
JAN 1974
$08 \ldots$
$<$
0.005
0.09
0.23
0.007
90
$<10$
$<0.01$
-. 0.24
-
- 100
20

01095220 STILLWATER RIVER NEAR STERLING, MASS. (LAT $422439 \mathrm{~N}$ LONG $0714730 \mathrm{~W}$ )

MAR 1973

JAN 1974

08 ...

0.004

\begin{tabular}{c|r}
$\ldots$ & 0.06 \\
$\cdots$ & $\ldots$
\end{tabular}

0.09

0.011

110

20

$<0.01$

0.44

$--$

-.

-.

80

30

01095400 QUINAPOXET R ABOVE SHAFT NO. 1 , AT OAKDALE, MASS. (LAT $422311 \mathrm{~N}$ LONG $0714823 \mathrm{~W}$ ) MAR 1973

MAR 1973

JAN 1974

08 ...

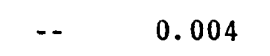

0.004

0.01

- 0.30

-.

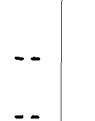

0.10

0.17

0.013

170

40

01095520 SOUTH BRANCH NASHUA RIVER AT SOUTH LANCASTER, MASS. (LAT 4226 18N LONG $0714054 \mathrm{~W}$ )

\begin{tabular}{|c|c|c|c|}
\hline $\begin{array}{l}\text { MAR } 1973 \\
21 . \ddot{1} \\
\text { JAN } 1974\end{array}$ & $\cdots$ & 0.005 & $\cdots$ \\
\hline $09 \ldots$ & 0.24 & - & 2.10 \\
\hline
\end{tabular}


Table 7.--Chemical analyses of surface water (Continued)

\begin{tabular}{|c|c|c|c|c|c|c|c|c|c|c|}
\hline TE & TIME & $\begin{array}{l}\text { DIS- } \\
\text { CHARGE, } \\
\text { INSTAN- } \\
\text { TANEOUS } \\
\left(\mathrm{FT}^{3} / \mathrm{s}\right)\end{array}$ & $\begin{array}{l}\text { SPE- } \\
\text { CIFIC } \\
\text { CON- } \\
\text { DUCT- } \\
\text { ANCE } \\
\text { (US/CM) }\end{array}$ & $\begin{array}{c}\text { PH } \\
\text { ( STAND- } \\
\text { ARD } \\
\text { UNITS) }\end{array}$ & $\begin{array}{l}\text { TEMPER- } \\
\text { ATURE } \\
\text { (DEG C) }\end{array}$ & $\begin{array}{l}\text { HARD - } \\
\text { NESS } \\
\text { (MG/L } \\
\text { AS } \\
\text { CAC03) }\end{array}$ & $\begin{array}{c}\text { HARD- } \\
\text { NESS, } \\
\text { NONCAR- } \\
\text { BONATE } \\
\text { (MG/L } \\
\text { CACO3) }\end{array}$ & $\begin{array}{l}\text { CALCIUM } \\
\text { DIS- } \\
\text { SOLVED } \\
\text { (MG/L } \\
\text { AS CA) }\end{array}$ & $\begin{array}{l}\text { MAGNE- } \\
\text { SIUM, } \\
\text { DIS- } \\
\text { SOLVED } \\
\text { (MG/L } \\
\text { AS MG) }\end{array}$ & $\begin{array}{c}\text { SODIUM, } \\
\text { DIS- } \\
\text { SOLVED } \\
\text { (MG/L } \\
\text { AS NA) }\end{array}$ \\
\hline
\end{tabular}

01095880 NONACOICUS BROOK NEAR AYER, MASS. (LAT $4233 \quad 43 \mathrm{~N}$ LONG $07136 \quad 39 W$ )

\begin{tabular}{|c|c|c|c|c|c|c|c|c|c|}
\hline $\begin{array}{l}\text { MAR } \\
20 . \\
\text { JAN } 1974\end{array}$ & 1530 & 115 & 125 & 6.6 & 5.0 & 28 & 16 & 8.8 & 1.4 \\
\hline $09 \ldots$ & 1415 & e30 & 148 & 6.8 & .0 & 30 & 17 & 9.0 & 1.8 \\
\hline
\end{tabular}

01095915 MULPUS BROOK NEAR SHIRLEY, MASS. (LAT 4234 26N LONG $0713443 W$ )

\begin{tabular}{|c|c|c|c|c|c|c|c|c|c|c|}
\hline $\begin{array}{l}20 \\
\text { JAN } 1974\end{array}$ & 1630 & 128 & 89 & 6.5 & 4.0 & 17 & 10 & 5.5 & 0.9 & 9.6 \\
\hline $09 \ldots$ & 1310 & 30 & 100 & 6.4 & .0 & 18 & 12 & 5.1 & 1.3 & $\cdot 10$ \\
\hline
\end{tabular}

01095920 NASHUA RIVER NEAR AYER, MASS. (LAT $4234 \quad 42 \mathrm{~N}$ LONG $07136 \quad 35 \mathrm{~W}$ )

MAR 1973

JAN 1974

JAN 197

$1415 \quad 1130$

116

6.5

5.0

23

$13 \quad 7.5$

$1.1 \quad 12$

$1250 \quad 290$

157

6.4

.0

29

$14 \quad 8.7$

1.8

16

01096050 SQUANNACOOK RIVER NEAR SHIRLEY, MASS. (LAT $423456 \mathrm{~N}$ LONG 0713637 W)

MAR 1973
20

$1700 \quad$ e600

50

6.3

4.0

9

$\begin{array}{ll}7 & 2.8\end{array}$

0.5

5.3

$09 .$. .

1150 e150

74

6.4

.0

13

$7 \quad 3.9$

.8

7.1

01096500 NASHUA RIVER AT EAST PEPPERELL, MASS. (LAT $424003 \mathrm{~N}$ LONG $0713432 \mathrm{~W}$ )

MAR 1973

21.974

$10 .$. .

$1520 \quad 1720$

112

$1030 \quad 330$

132

6.5

6.0

20

6.5

1.0

25

9
12

1.0

$1.6 \quad 13$

01096503 NISSITISSIT RIVER AT PEPPERELL, MASS. (LAT $42 \quad 40$ 19N LONG $0713439 \%$ )

MAR 1973

$\begin{array}{rl}22 & 1973 \\ \text { JAN } & 1974\end{array}$

$1015 \quad 303$

52

6.9

$2.0 \quad 16$

6.6

.0

16

9
8

4. 9

0.8

4.5

1045

63

8

4.4

1.1

4.7

01096505 UNKETY BROOK NEAR PEPPERELL, MASS. (LAT $424123 \mathrm{~N}$ LONG $0713254 \mathrm{~W}$ )

MAR 1973
21.9
JAN 1974

10...

$1630 \quad 27$

62

6.5

4.0

6.6

.0

21

13

7.0

0.8

3.7

See footnotes at end of table. 
Table 7.--Chemical analyses of surface water (Continued)

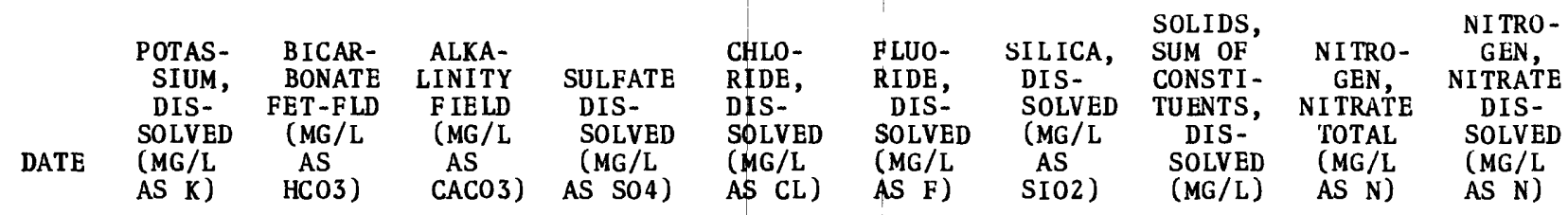

01095880 NONACOICUS BROOK NEAR AYER, MASS, (LAT 4233 43N LONG $07136 \quad 39$ )

MAR 1973

20...

JAN 1974

$09 .$.

MAR 1973

20...

JAN 1974

09...

MAR 1973

21...

JAN 1974

09...

MAR 1973

20...

JAN 1974

$09 .$.
21

24

1.3

16

13

13

0.1

2.5

3.2

67

75

0.20

01095915 MULPUS BROOK NEAR SHIRLEY, MASS.' (LAT 4234 26N LONG $0713443 W$ )
1.0
8
9.5
15
$<0.1$
4.4
51
.1
4.8
52
0.19
9.816

01095920

4234

$42 N$ LONG 0713635 )
1.5
12
$10 \quad 14$
18
0.1
5.7
69
--
0.70
2.0
18
$15 \quad 16$
23
.2
6.4
$83 \quad 1.29$
01096050 SQUANNACOOK RIVER NEAR SHIRLEY, MASS. (LAT 4234 56N LONG 0713637 W)
0.6
3
2
7.2
8.0
0.1
5.3
32
-
0.20
.9
7
68.1
9.9
.1
7.1
41
0.34

01096500 NASHUA RIVER AT EAST PEPPERELL, MASS. (LAT $424003 \mathrm{~N}$ LONG $0713432 \mathrm{~W}$ )

MAR 1973

JAN 1974

JAN...
1.4

$$
14
$$

$\begin{array}{ll}11 & 15 \\ 13 & 13\end{array}$
16
0.3
6.0
70
--
0.80
1.6
16
19
.2
6.4
70
0.96

01096503 NISSITISSIT RIVER AT PEPPERELL, MASS. (LAT 4240 19N LONG $0713439 \mathrm{~W}$ )

MAR 1973

22 ...

JAN 1974

10 ...

$\begin{array}{cccc}0.5 & 8 & 7 & 7.7 \\ .6 & 10 & 8 & 7.6 \\ 01096505 & \text { UNKETY } & \text { BROOK NEAR } & \text { PEPPER } \\ 1.1 & 10 & 8 & 11 \\ 1.3 & 13 & 11 & 14\end{array}$

7.9

0.1

5.5

37

6.5

37

0.27

0.20

.2

23N LONG $0713254 W$ )

MAR 1973

JAN 1974

10...

$\begin{array}{c:cc}7.0 & <0.1 & 6.1 \\ 8.0 & .1 & 10\end{array}$

42

- $\quad 0.10$

See footnotes at end of table. 
Table 7.--Chemical analyses of surface water (Continued)

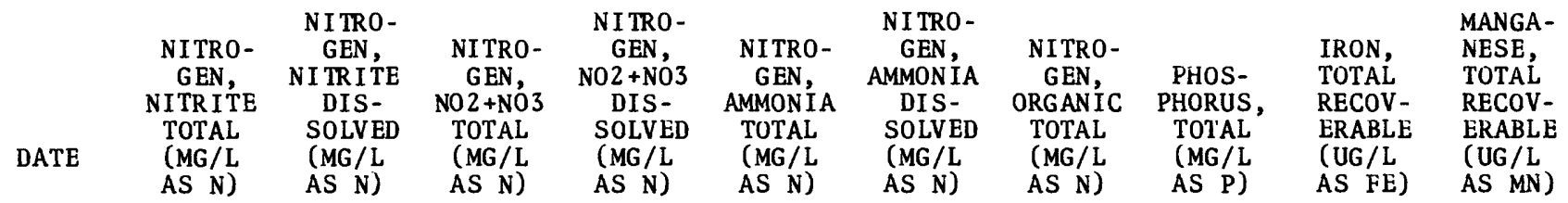

01095880 NONACOICUS BROOK NEAR AYER, MASS. (LAT 4233 43N LONG $0713639 \mathrm{~W}$ )

MAR 1973

$20 .$.

JAN 1974

$09 .$.

$\begin{array}{rrrrrrrrrr}-- & 0.007 & -- & & -- & 0.08 & 0.82 & 0.014 & 210 & 30 \\ 0.01 & -- & 0.21 & -- & -- & -- & -- & \ldots & 230 & 60\end{array}$

01095915 MULPUS BROOK NEAR SHIRLEY, MASS. (LAT 4234 26N LONG 07134 43W)

MAR 1973

JAN 1974

$09 .$. .
$-0.008$
0.07
0.62
0.006
130
20
0.01
0.20
$--$
- -
-.
80
40

01095920 NASHUA RIVER NEAR AYER, MASS. (LAT $423442 \mathrm{~N}$ LONG 0713635 )

MAR 1973

21 .

JAN 1974

$09 .$. .

$\begin{array}{rrrrrrrrrr}- & 0.003 & - & - & -- & 0.08 & 0.65 & 0.18 & 460 & 100 \\ 0.01 & -- & 1.30 & -- & -- & \ldots & -. & \ldots & 600 & 150\end{array}$

01096050 SQUANNACOOK RIVER NEAR SHIRLEY, MASS. (LAT $423456 \mathrm{~N}$ LONG 0713637 )

MAR 1973

20...

JAN 1974

$09 .$. .

$\begin{array}{rrrrrrrrrr}-- & 0.007 & -. & -- & -- & 0.10 & 0.2 & 0.017 & 110 & 40 \\ 0.01 & -- & 0.35 & -- & 0.49 & -- & -. & -- & 170 & 80\end{array}$

01096500 NASHUA RIVER AT EAST PEPPERELL, MASS. (LAT $424003 \mathrm{~N}$ LONG $0713432 \mathrm{~W}$ )

MAR 1973

$21 \ldots$.

JAN 1974

10 ...

$\begin{array}{rrrrrrrrrr}\ldots & 0.004 & \ldots & \ldots & \ldots & 0.12 & 0.86 & 2.20 & 470 & 100 \\ 0.04 & \ldots & 1.00 & \ldots & \ldots & \ldots & \ldots & \ldots & 500 & 130\end{array}$

01096503 NISSITISSIT RIVER AT PEPPERELL, MASS. (LAT 4240 19N LONG $0713439 \mathrm{~W})$

MAR 1973

22.

JAN 1974

$10 .$. .

$\begin{array}{rrrrrrrrrr}- & 0.005 & \ldots & \ldots & \ldots & 0.08 & 0.11 & 0.005 & 110 & 20 \\ 0.01 & \ldots & 0.28 & -- & -- & -- & \ldots & \ldots & 120 & 40\end{array}$

01096505 UNKETY BROOK NEAR PEPPERELL, MASS. (LAT $424123 \mathrm{~N}$ LONG $0713254 \mathrm{~W}$ )

MAR 1973

JAN 1974

$10 .$. .

$\begin{array}{rrrrrr}\therefore & 0.007 & - & \ldots & - & 0.06 \\ 0.01 & \ldots & 0.34 & \ldots & \ldots & \ldots\end{array}$

$0.21 \quad 0.013$

160

$<10$

e Estimated value.

< Actual value is known to be less than the value shown. 
Table 8.-Hydrologic-data reports for Massachusetts

An asterisk indicates that the report is out of print but may be consulted at the offices of the U.S. Geological Survey, 10 Causeway Street, Boston, Massachusetts, and at many public and educational institution libraries.

*1 Wilmington-Reading area, by John A. Baker and Edward A. Sammel, 1961, 50 p., 2 figs. Covers an area of about 43 square miles in the upper part of the Ipswich River basin in nor theastern Massachusetts.

*2 Lower Ipswich River basin, by Edward A. Sammel and John A. Baker, 1962, 47 p., 2 figs. Covers an area of about 110 square miles in nor the astern Massachusetts.

*3 Lowell area, by John A. Baker and Richard G. Petersen, 1962, 28 p., 2 figs. Covers an area of about 115 square miles and includes most of the metropolitan area of the City of Lowell.

*4 Parker and Rowley River basins, by Edward A. Sammel, 1962, 33 p., 2 figs. The rivers drain an area of about 77 square miles in nor the astern Massachusetts.

*5 Brockton-Pembroke area, by Richard G. Petersen, 1962, 46 p., 2 figs. Covers an area of about 112 square miles in the nor thern part of Plymouth County.

*6 Western Massachusetts, by Richard G. Petersen and Anthony Maevsky, 1962, 21 p., $1 \mathrm{fig}$. Covers an area of about 2,865 square miles and includes all of Berkshire, Franklin, Hampshire, and Hampden Counties.

*7 Southeastern Massachusetts, by Anthony Maevsky and Janet A. Drake, 1963, 55 p., 2 figs. Covers an area of about 1,930 square miles and includes all of Barnstable, Bristol, Dukes, Nantucket, and Plymouth Counties (exclusive of the BrocktonPembroke Area).

*8 Assabet River basin, by Samuel J. Pollock and William B. Fleck, 1964, 45 p., 1 pl. Covers an area of approximately 177 square miles and includes parts of Middlesex and Worcester Counties.

*9 Housatonic River basin, by Ralph F. Norvitch and Mary E. S. Lamb, 1966, 50 p., 1 pl. Covers an area of about 530 square miles in the upper part of the basin, which is nor th of the Connecticut-Massachusetts State line.

*10 Nor thern part, Ten Mile and Taunton River basins, by John R. Williams and Richard E. Willey, 1967, 56 p., 1 pl., 1 fig. Covers an area of about 195 square miles within Bristol, Norfolk, and Plymouth Counties.

*11 Millers River basin, by Donald R. Wiesnet and William B. Fleck, 1967, 29 p., 1 pl., $1 \mathrm{fig}$. Covers an area of about 392 square miles within Franklin and Worcester Counties, Massachusetts, and Hillsborough and Cheshire Counties, New Hampshire.

*12 Taunton River basin, by John R. Williams and Richard E. Willey, 1970, 102 p., 1 pl., $1 \mathrm{fig}$. Covers an area of about 528 square miles in Bristol, Norfolk, and Plymouth Counties.

*13 Deerfield River basin, by Bruce P. Hansen, Frederick B. Gay, and L. G. Toler, 1973, 59 p., 1 fig., 1 pl. Covers an area of 348 square miles in nor thwestern Massachusetts.

*14 Neponset and Weymouth River basins, by R. A. Brackley, William B. Fleck, and Richard E. Willey, 1973, 51 p., 1 fig., 1 pl. Covers an area of 183 square miles in eastern Massachusetts south of Boston. 
Table 8.-Hydrologic-data reports for Massachusetts (Continued)

*15 Hoosic River basin, by Bruce P. Hansen, Frederick B. Gay, and L. G. Toler, 197 4, 33 p., 1 pl., 1 fig. Covers an area of 164 square miles in northwestern Massachusetts.

* 16 Weir River, Hingham, to Jones River, Kingston, by John R. Williams, Richard E. Willey, and Gary D. Tasker, 1975, 63 p., 1 pl., 1 fig. Principal basins covered are those of Weir River, James Brook, Bound Brook, North River, South River, and Jones River.

*17 Ground-water levels in Massachusetts, 1936-74, by Anthony Maevsky, 1976, 107 p., 2 figs. Documents both short-term and long-term ground-water-level trends in typical hydrologic situations and different geographic areas of the Com monwealth.

*18 Plymouth to Weweantic River, Wareham, by John R. Williams, Gary D. Tasker, and Richard E. Willey, 1977, 31 p., 1 pl., 1 fig. Principal basins are Town Brook, Eel River, and Beaverdam Brook, all draining to Cape Cod Bay; Herring Brook draining to the Cape Cod Canal; and Red Brook, Agawam River, Wankinco River, and Weweantic River, all draining to Buzzards Bay.

*19 Charles River basin, by Eugene H. Walker, William W. Caswell, and S. William Wandle, Jr., 1977, 53 p., 1 pl., 1 fig. Covers an area of about 300 square miles of eastern and southeastern Massachusetts within the counties of Middlesex, Norfolk, Suffolk, and Woreester.

* 20 Northwest Shore of Buzzards Bay, by John R. Williams, Richard E. Willey, and Gary D. Tasker, 1980, 30 p., 1 pl. Principal drainage basins are Sipican River, Aucoot Brook, Mat tapoisett River, Acushnet River, and Paskamansett River.

21 Coastal drainage basins of nor the astern Massachusetts, from Castle Neck River, Ipswich, to Mystic River, Boston, by David F. Delaney and Frederick B. Gay, 1980, 40 p., 1 pl. Principal streams are the Annisquam, Castle Neck, Danvers, Essex, Mystic, and Saugus Rivers, which flow into Ipswich and Massachusetts Bays.

22 Shawsheen River basin by David F. Delaney and Frederick B. Gay, 1981, 22 p., 1 pl. Principal tributaries are Content, Elm, Heath, Hussey, Kiln, Rogers, Spring, Strong Water, Vine and Webb Brooks.

23 Lake Cochituate drainage basin, Framingham-Natick, Massachusetts, by Frederick B. Gay, 1981, 61 p., 1 pl. Covers 17.7 square miles above the outlet of Lake Cochituate which includes Beaverdam, Course, Pegan, and Snake Brooks, and Fisk Pond.

24 Lower Mer rimack River basin, from Concord River, Lowell, to Plum Island, Newburyport, by David F. Delaney and Frederick B. Gay, 1981, 34 p., 1 pl. Principal tributaries are Bare Meadow, Bartlett, Cobbler Creek, Fish, Richardson, and Trull Brooks, Artichoke, East Meadow, Indian, Little, Powwow, and Spicket Rivers, and Johnson Creek. The Blackwater River basin is included in the report.

25 Southeastern Massachusetts, Narragansett Bay, and Rhode Island Sound, by Richard E. Willey, John R. Williams, and Gary D. Tasker, 1983, 42 p., 1 pl. Principal drainage basins are those of the East and West Branches of the Westport River which empty into Rhode Island Sound and of the Lee, Cole, Kickamuit, Palmer, and Runnins Rivers draining to Narragansett Bay. The area includes a small part of the Tenmile River basin not included in previous reports.

26 Ground-water and pond levels, Cape Cod, Massachusetts, 1950-82, by Diane F. Letty, 1984, 81 p. Contains water-level measurements from a network of 68 observation wells and pond levels for 10 ponds located throughout Cape Cod. 